Florida International University

FIU Digital Commons

FIU Electronic Theses and Dissertations

University Graduate School

$11-9-2017$

\title{
Advanced Solutions for Renewable Energy Integration into the Grid Addressing Intermittencies, Harmonics and Inertial Response
}

Arash Anzalchi

Florida International University, aanza005@fiu.edu

DOI: 10.25148 /etd.FIDC004064

Follow this and additional works at: https://digitalcommons.fiu.edu/etd

Part of the Power and Energy Commons

\section{Recommended Citation}

Anzalchi, Arash, "Advanced Solutions for Renewable Energy Integration into the Grid Addressing Intermittencies, Harmonics and Inertial Response" (2017). FIU Electronic Theses and Dissertations. 3506.

https://digitalcommons.fiu.edu/etd/3506 


\section{FLORIDA INTERNATIONAL UNIVERSITY}

Miami, Florida

\section{ADVANCED SOLUTIONS FOR RENEWABLE ENERGY INTEGRATION INTO THE GRID ADDRESSING INTERMITTENCIES, HARMONICS AND INERTIAL RESPONSE}

A dissertation submitted in partial fulfillment of the requirements for the degree of DOCTOR OF PHILOSOPHY in ELECTRICAL ENGINEERING

by

Arash Anzalchi 
To: Dean John L. Volakis

College of Engineering and Computing

This dissertation, written by Arash Anzalchi, and entitled Advanced Solutions for Renewable Energy Integration into the Grid Addressing Intermittencies, Harmonics and Inertial Response, having been approved in respect to style and intellectual content, is referred to you for judgment.

We have read this dissertation and recommend that it be approved.

Osama A. Mohammed

Nezih Pala

Sakhrat Khizroev

Saroj K. Biswas

Arif I. Sarwat, Major Professor

Date of Defense: November 9, 2017

The dissertation of Arash Anzalchi is approved.

Dean John L. Volakis

College of Engineering and Computing

Andres G. Gil

Vice President for Research and Economic Development and Dean of the University Graduate School

Florida International University, 2017 
(C) Copyright 2017 by Arash Anzalchi

All rights reserved. 


\section{DEDICATION}

This dissertation is dedicated to my devoted and loving wife, Maneli, and to my wonderful newborn daughter, Shailie. A special feeling of gratitude to my parents, Elahe and Reza, who have never left my side and are very special. I also dedicate this dissertation to my sister Elnaz who have supported me throughout the process. I dedicate this work and give special thanks to my parents in law, Alireza and Shery. 


\section{ACKNOWLEDGMENTS}

I would like to express my sincere gratitude to my principal advisor, Professor Arif Sarwat, for his guidance, support, encouragement, and thought-provoking discussions during my doctoral research. This dissertation would not have been possible without his help, support, resolute dedication, and patience. His passion for success has inspired me

to come up with new ideas. His expert advice and unsurpassed knowledge of the various fields of electrical energy systems has always provided me an endless supply of idea to move to the next step and complete this dissertation. I am also grateful for the chance he gave me to work under his supervision at his excellent research group at the Energy, power and Sustainability (EPS).

I thank the university graduate school for awarding me the Dissertation Year Fellowship (DYF) which significantly helped me complete this dissertation. I would like to also thank my dissertation committee members for their insightful comments and constructive suggestions in the review of my dissertation.

Also, I am thankful to all my colleagues in the EPS Laboratory. Many graduate and undergraduate student scholars have helped me with discussions, contributions, and assistance contributing to achieving my research goals. I also want to mention the support of our departmental staff, especially Ms. Pat Brammer and Mr. Oscar Silveira, who gave me great help and support.

Finally, I would like to give a special thanks to my wife for her everlasting love and to my mother and father for their unconditional support and love. I also extend my gratitude to my dearest sister, Elnaz, for her encouragement and assistance. 


\author{
ABSTRACT OF THE DISSERTATION \\ ADVANCED SOLUTIONS FOR RENEWABLE ENERGY INTEGRATION INTO \\ THE GRID ADDRESSING INTERMITTENCIES, HARMONICS AND INERTIAL \\ RESPONSE \\ by \\ Arash Anzalchi \\ Florida International University, 2017 \\ Miami, Florida \\ Professor Arif I. Sarwat, Major Professor
}

Numerous countries are trying to reach almost $100 \%$ renewable penetration. Variable renewable energy (VRE), for instance wind and PV, will be the main provider of the future grid. The efforts to decrease the greenhouse gasses are promising on the current remarkable growth of grid connected photovoltaic (PV) capacity. This thesis provides an overview of the presented techniques, standards and grid interface of the PV systems in distribution and transmission level. This thesis reviews the most-adopted grid codes which required by system operators on large-scale grid connected Photovoltaic systems. The adopted topologies of the converters, the control methodologies for active - reactive power, maximum power point tracking (MPPT), as well as their arrangement in solar farms are studied.

The unique L(LCL)2 filter is designed, developed and introduced in this thesis. This study will help researchers and industry users to establish their research based on connection requirements and compare between different existing technologies. Another, major aspect of the work is the development of Virtual Inertia Emulator (VIE) in combination of hybrid energy storage system addressing major challenges with VRE implementations.

Operation of a photovoltaic (PV) generating system under intermittent solar radiation is a challenging task. Furthermore, with high-penetration levels of photovoltaic en- 
ergy sources being integrated into the current electric power grid, the performance of the conventional synchronous generators is being changed and grid inertial response is deteriorating. From an engineering standpoint, additional technical measures by the grid operators will be done to confirm the increasingly strict supply criteria in the new inverter dominated grid conditions.

This dissertation proposes a combined virtual inertia emulator (VIE) and a hybrid battery-supercapacitor-based energy storage system . VIE provides a method which is based on power devices (like inverters), which makes a compatible weak grid for integration of renewable generators of electricity. This method makes the power inverters behave more similar to synchronous machines. Consequently, the synchronous machine properties, which have described the attributes of the grid up to now, will remain active, although after integration of renewable energies. Examples of some of these properties are grid and generator interactions in the function of a remote power dispatch, transients reactions, and the electrical outcomes of a rotating bulk mass.

The hybrid energy storage system (HESS) is implemented to smooth the short-term power fluctuations and main reserve that allows renewable electricity generators such as PV to be considered very closely like regular rotating power generators. The objective of utilizing the HESS is to add/subtract power to/from the PV output in order to smooth out the high frequency fluctuations of the PV power, which may occur due to shadows of passing cloud on the PV panels. A control system designed and challenged by providing a solution to reduce short-term PV output variability, stabilizing the DC link voltage and avoiding short term shocks to the battery in terms of capacity and ramp rate capability.

Not only could the suggested system overcome the slow response of battery system (including dynamics of battery, controller, and converter operation) by redirecting the power surges to the supercapacitor system, but also enhance the inertial response by emulating the kinetic inertia of synchronous generator. 


\section{TABLE OF CONTENTS}

1. INTRODUCTION ...................... 1

1.1 General Statement of Problem Area . . . . . . . . . . . . . . . . . . 1

1.2 Literature Review . . . . . . . . . . . . . . . . . . . . . . . . . 5

1.3 Motivation and Purpose of Research . . . . . . . . . . . . . . . 8

1.4 Research Objectives and Original Contributions . . . . . . . . . . . . . 10

1.5 Dissertation Organization . . . . . . . . . . . . . . . . . . . . . 13

2. INVESTIGATION OF STANDARDS AND THRESHOLD ISSUES AFFECTING PERFORMANCE OF GRID-CONNECTED PHOTOVOLTAIC SYSTEMS 16

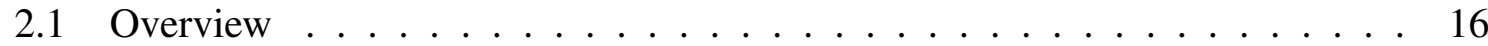

2.2 Problem Statement . . . . . . . . . . . . . . . . . . . . . . . . . 17

2.3 Solution Approach . . . . . . . . . . . . . . . . . . . . . . . . . . . 18

2.4 Challenges to Integrate Solar Photovoltaic . . . . . . . . . . . . . . . . . 18

2.5 Grid Codes Requirements . . . . . . . . . . . . . . . . . . . . . . . . 23

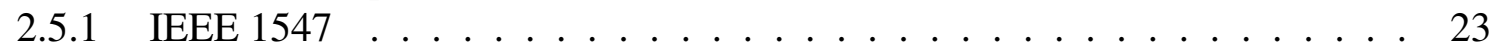

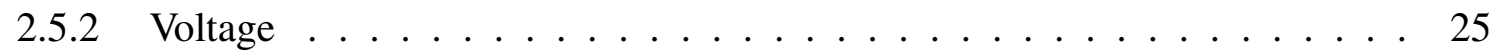

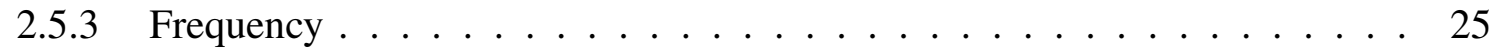

2.5 .4 Power Quality . . . . . . . . . . . . . . . . . . . . . . 26

2.5 .5 Islanding . . . . . . . . . . . . . . . . . . . . . 27

2.6 Grid codes in Different Countries . . . . . . . . . . . . . . . . . . . . . . 28

2.7 Topology Classification of Inverters $\ldots \ldots \ldots$

2.8 Three Phase Grid Connected Inverters ～. . . . . . . . . . . . . . . . . . . . 39

2.9 Control of Grid Connected PV Systems ～. . . . . . . . . . . . . . . . . . 43

2.9.1 Renewable side controllers . . . . . . . . . . . . . . . . . . . . . . 43

2.9.2 Grid-side controllers . . . . . . . . . . . . . . . . . . . . . . . . . . . 44

2.9 .3 Control Strategies . . . . . . . . . . . . . . . . . . . . . . . . . . . . . 47

2.10 Energy Storage in Photovoltaic Systems . . . . . . . . . . . . . . . . 52

2.11 Future Applications of PV Renewable Energy . . . . . . . . . . . . . . 57

2.12 Summary . . . . . . . . . . . . . . . . . . 58

3. ANALYSIS OF CARBON TAX AS AN INCENTIVE TOWARD BUILDING SUSTAINABLE GRID WITH RENEWABLE ENERGY UTILIZATION . . . 60

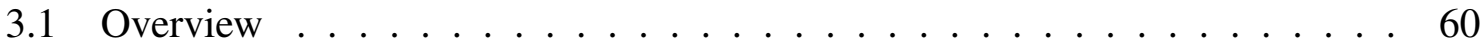

3.2 Problem Statement . . . . . . . . . . . . . . . . . . . . . 61

3.3 Solution Approach $\ldots \ldots \ldots$. . . . . . . . . . . . . . . 62

3.4 Modeling the Proposed System . . . . . . . . . . . . . . . . . . 63

3.5 Data Inputs . . . . . . . . . . . . . . . . . . . . . . . . . 65

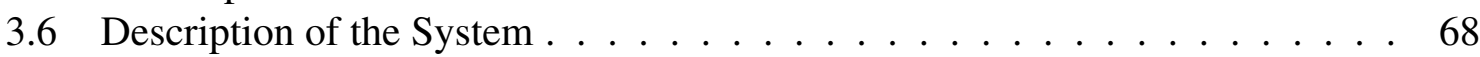

3.6 .1 Wind Turbine . . . . . . . . . . . . . . . . . . . . . 68

3.6 .2 Photovoltaic panel . . . . . . . . . . . . . . . . . . . . . . . . . 69 
3.6.3 Electric Grid . . . . . . . . . . . . . . . . . . . . . . . . . . . . . . . . . . 69

3.6 .4 Converter . . . . . . . . . . . . . . . . . . . . . . . . . . . . 69

3.7 Simulation Results . . . . . . . . . . . . . . . . . . . . . . . . 70

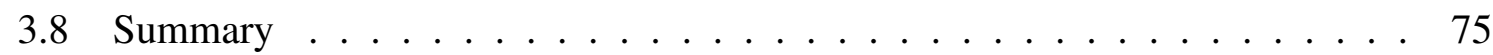

4. ARTIFICIAL NEURAL NETWORK BASED DUTY CYCLE ESTIMATION FOR MAXIMUM POWER POINT TRACKING IN PHOTOVOLTAIC SYSTEM . 77

4.1 Overview . . . . . . . . . . . . . . . . . . . . . . 77

4.2 Problem Statement . . . . . . . . . . . . . . . . . . . . . . . 77

4.3 Solution Approach . . . . . . . . . . . . . . . . . . . . . . . . . 79

$4.3 .1 \quad$ PV array characteristics $\ldots \ldots \ldots \ldots$. . . . . . . . . . 80

4.3.2 Neural Network Architecture . . . . . . . . . . . . . . . . . . . . . . . 82

4.4 Results and Discussions . . . . . . . . . . . . . . . . . . . . . . . . . . . 84

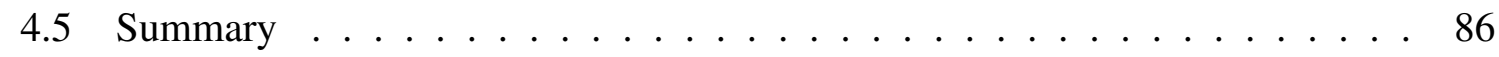

5. ANALYZING THE TRENDS OF VOLTAGE FLUCTUATIONS, HARMONICS, FREQUENCY, AND LEGACY DEVICES OF THE PHOTOVOLTAIC TEST-

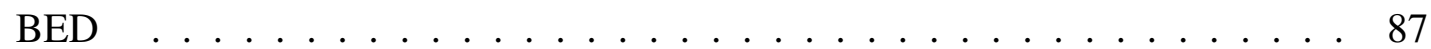

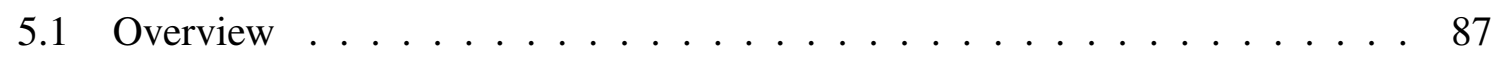

5.2 Problem Statement . . . . . . . . . . . . . . . . . . . . . . . . . . 88

5.3 Solution Approach . . . . . . . . . . . . . . . . . . . . . . . 90

5.4 Test-bed Description and Case Study . . . . . . . . . . . . . . . . . . 91

$5.4 .1 \quad$ Placement of Revolution . . . . . . . . . . . . . . . . . . . . . 92

5.5 Results and Analysis . . . . . . . . . . . . . . . . . . . . . . . 92

$5.5 .1 \quad$ Power Quality Study . . . . . . . . . . . . . . . . . . . . 93

5.5.2 Voltage Measurement . . . . . . . . . . . . . . . . . . . . . . . 99

5.5 .3 Voltage Profile Analysis . . . . . . . . . . . . . . . . . . . . 100

5.6 Steady-State Scenario Study . . . . . . . . . . . . . . . . . . . 101

5.6 .1 Voltage Deviation Study: . . . . . . . . . . . . . . . . . . . . . 103

5.6 .2 Feeder Loss Study: . . . . . . . . . . . . . . . . . . . . . . . . . . . . . . 106

5.6 .3 Time-Series Scenario Study . . . . . . . . . . . . . . . . . . . . 106

5.7 Summary . . . . . . . . . . . . . . . . . . . . . . 110

6. A NEW TOPOLOGY OF HIGHER ORDER POWER FILTER FOR SINGLEPHASE GRID-TIED VOLTAGE SOURCE INVERTERS . . . . . . . . . . 112

6.1 Overview . . . . . . . . . . . . . . . . . . . . . . 112

6.2 Problem Statement . . . . . . . . . . . . . . . . . . . . . . . . . 113

6.3 Solution Approach . . . . . . . . . . . . . . . . . . . . . . . . . . . . 114

6.4 Principles of The $L L C L$-Filters $\ldots \ldots \ldots$

6.5 Proposed $L(L C L)_{2}$ Filter $\ldots \ldots \ldots \ldots$

6.5.1 Analysis of ripple current . . . . . . . . . . . . . . . . . . . . 120

6.5.2 Parameter Design of the $L(L C L)_{2}$ Filter . . . . . . . . . . . . . . 122

6.5.3 Design example . . . . . . . . . . . . . . . . . . . 125 
6.5.4 Size Estimation of $L L C L$ and $L(L C L)_{2}$ Filters . . . . . . . . . . . . 127

6.5.5 Efficiency comparison between the $L L C L$ and $L(L C L)_{2}$ Filters . . . . . 128

6.5.6 Optimization of The $L(L C L)_{2}$ Parameters . . . . . . . . . . . . . . . . . 129

6.5.7 Close-Loop Stability Analysis . . . . . . . . . . . . . . . . . . . . 131

6.6 Simulation and Experimental Results . . . . . . . . . . . . . . . . . . . . . 134

6.7 Analysis and Discussion . . . . . . . . . . . . . . . . . . . 140

6.7.1 Power Loss Analysis of the $L(L C L)_{2}$ Filter components . . . . . . . . . 142

6.7 .2 Inverter Loss Analysis . . . . . . . . . . . . . . . . . . . . . . . . . . 144

6.7.3 Small-signal model of the dc-link dynamics considering the instantaneous power for bi-directional ac/dc power flow applications . . . . . 145

6.8 Summary . . . . . . . . . . . . . . . . . . . . . . . . 150

7. A COMBINATORIAL ADVANCED SOLUTION FOR PV INTEGRATION TO ADDRESS INTERMITTENCY AND PROVIDE INERTIAL RESPONSE . . 152

7.1 Overview . . . . . . . . . . . . . . . . . . . . . . 152

7.2 Problem Statement . . . . . . . . . . . . . . . . . . . . . . . . . . 154

7.3 Solution Approach . . . . . . . . . . . . . . . . . . . . . 157

7.4 Technical Realization . . . . . . . . . . . . . . . . . . . . . . . . . . . 159

7.5 Different Virtual Synchronous Machine algorithms . . . . . . . . . . . . 160

7.5 .1 VSG topology of VSYNC Project . . . . . . . . . . . . . . . . 161

7.5 .2 VSG topology by IEPE . . . . . . . . . . . . . . . . . . . . 163

7.5 .3 VSG topology by ISE lab . . . . . . . . . . . . . . . . . . . . 165

7.5.4 VSG topology by KHI . . . . . . . . . . . . . . . . . . . . . 165

7.5.5 Typical Method of Implementation of Virtual Inertia Emulator in Previous

Studies . . . . . . . . . . . . . . . . . 167

7.6 VIE Implementation $\ldots \ldots \ldots$

7.6 .1 Load frequency control . . . . . . . . . . . . . . . . . . . . . . . 169

7.6 .2 System Modeling . . . . . . . . . . . . . . . . . . . . . . . 171

7.7 Power Smoothing and HESS . . . . . . . . . . . . . . . . . . . . 177

7.8 Modeling of the Energy Storage System . . . . . . . . . . . . . . . . 179

7.8.1 Modeling the Supercapacitor Bank . . . . . . . . . . . . . . . . . 179

7.8.2 Modeling of the Battery Bank . . . . . . . . . . . . . . . . . . 182

7.9 Design of Controllers for the HESS . . . . . . . . . . . . . . 183

7.9.1 Design of Current Control Loop of Battery . . . . . . . . . . . . . . 185

7.9.2 Design of Current Control Loop of SC . . . . . . . . . . . . . . . . . . . 185

7.9 .3 Design of Rate Limiter . . . . . . . . . . . . . . . . . . . . . . . 186

7.10 System Description . . . . . . . . . . . . . . . . . . . . . 187

7.11 Simulation Results . . . . . . . . . . . . . . . . . . . . . . . . . . 188

7.11.1 Performance of HESS in high ramp PV output fluctuations . . . . . . . . 190

7.11.2 Dynamic operation of Virtual Inertia Emulator . . . . . . . . . . . 191

7.12 Summary . . . . . . . . . . . . . . . . . . . . . 196 
8. CONCLUSION AND FUTURE WORK . . . . . . . . . . . . . . . . . . . . . . . . . . . . . .

8.1 Conclusion . . . . . . . . . . . . . . . . . . 198

8.2 Future Work . . . . . . . . . . . . . . . . 202

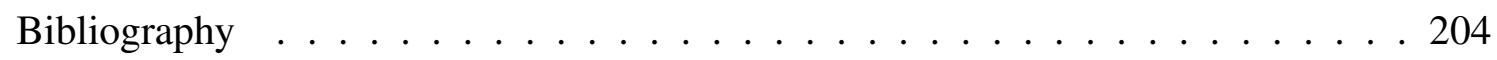

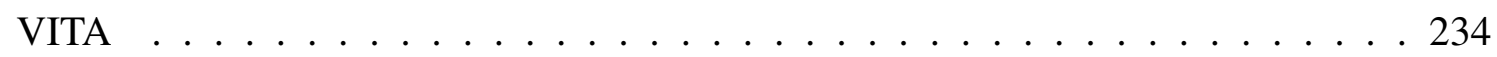




\section{LIST OF TABLES}

TABLE

PAGE

2.1 Technical Challenges in Regards to Implementation of Renewable Energy to the Smart Grid . . . . . . . . . . . . . . . . . . 20

2.2 Required Fundamentals for Grid Integration of Renewable Energies. . . . . . 22

2.3 ANSI C84.1 Voltage Ranges for 120V Base.[1] . . . . . . . . . . . . . 24

2.4 Interconnection system response to abnormal Voltages [1] . . . . . . . . 25

2.5 Interconnection system response to abnormal Frequencies [1] . . . . . . . 26

2.6 Maximum Harmonic Current Distortion in Percent of Current [1] . . . . . . 26

2.7 LVRT requirement in China grid. . . . . . . . . . . . . . 31

2.8 Examples of standards and grid codes for DER integration . . . . . . . . 33

2.9 Comparisons of multi-functional three-phase grid-inverter topologies . . . . 42

2.10 Comparisons of multi-functional single-phase grid-inverter topologies. . . . 43

2.11 Examples of Different MPPT Methods . . . . . . . . . . . . . 45

3.1 History and Forecast of Energy Consumption and Number of Rural and Residential Customers [2] . . . . . . . . . . . . . 66

3.2 Cost Summary of the System . . . . . . . . . . . . . . . 70

3.3 Variation of NPC and COE by Tax on Co2 for Two Scenarios . . . . . . 70

3.4 GHG Emissions for Two Scenarios . . . . . . . . . . . . . . 71

4.1 Specification of Sunpower SPR-305 PV Module on Standard Test Conditions $\left(1000 \mathrm{~W} / \mathrm{M}^{2}, 25^{\circ} \mathrm{C}\right) \ldots \ldots \ldots$. . . . . . . . . . 82

4.2 Training Parameter Values . . . . . . . . . . . . . . . . . 84

5.1 Low-voltage System Classification and Distortion Limits [3] . . . . . . . . 90

5.2 Maximum Current Distortion in Percent of Current [3] . . . . . . . . . . . . 90

5.3 Total Numbers of Switching Operations. . . . . . . . . . . . . . . 110

6.1 Size Estimation of $L L C L$ and $L(L C L)_{2}$ Filters . . . . . . . . . . . . 127

6.2 Calculated Power Losses And Efficiency of The $L(L C L)_{2}$ Filter and the LLCL Filters Based on Nominal Output Power of 700w. . . . . . . . . . 129 
6.3 Designed Parameters for Experimental Setup . . . . . . . . . . . . . . . 132

6.4 Parameters Which Are Used for Theoretical Converter Loss Calculation. . . 143

6.5 Power Losses of the $L(L C L)_{2}$ Filter and The $L L C L$ Filter. . . . . . . . . . 145

7.1 Performance of Battery vs SC . . . . . . . . . . . . . 177

7.2 Supercapacitor bank Specification. . . . . . . . . . . . . . . . . 182

7.3 Parameters of the sixth order model of the $6.4 \mathrm{~F}$ supercapacitor bank. . . . . 182

7.4 Parameters of the battery model. . . . . . . . . . . . . . . . . 183 


\section{LIST OF FIGURES}

FIGURE

PAGE

1.1 Typical schematic of a $180 \mathrm{~kW}$ photovoltaic system for industrial locations. . 3

1.2 Two-way coupled Transmission and Distribution grid . . . . . . . . . . 6

1.3 General configuration and concept of the VIE . . . . . . . . . . 8

2.1 Challenges that arise when integrating renewable resources into the smart grid. 19

2.2 Fault Ride Through Germany . . . . . . . . . . . . . . . . . . 29

2.3 Dynamic response of Germanny's power grid. . . . . . . . . . . . . . . 30

2.4 Fault Ride Through Requirements in Different Countries: (a) Denmark, (b) France, (c) Italy, (d) England, (e) Spain . . . . . . . . . . . . . . . . . . 34

2.5 Summary of PV inverters technologies. (a) centralized (b) string (c) multistring (d) ac-module and ac cell technologies. . . . . . . . . . 36

2.6 (a) Single power processing stage (For voltage amplification, grid current control, and MPPT) (b) Dual power processing inverter (dcac inverter controls the grid current, the dcdc converter is in charge of the MPPT, the Voltage amplification can be included in both stages. (c) Dual-stage inverter. . . . . . . . . . . . . . . . .

2.7 Power decoupling capacitor locations: (a) In parallel with the PV modules (in single-stage inverter applications). (b) in parallel with the dc-link or the PV modules (in multi-stage inverter applications) . . . . . . . . .

2.8 Examples of transformer-included inverter solutions. (a) Line-frequency transformer (LFT) is placed between the grid and the inverter (solves problems with injection of dc currents into the grid). (b) High-frequency transformer (HFT) is embedded in an HF-link grid-connected ac/ac inverter. (c) HFT is embedded in a dc-link PV-module-connected dcdc

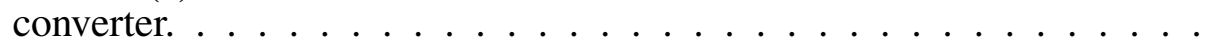

2.9 High-input-voltage transformerless PV inverter (with common-mode (CM)

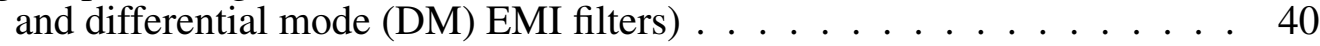

2.10 Topology and its of the MFGCI cell (a) application in three-phase utility (b) application in three-phase utility . . . . . . . . . . . . . . 41

2.11 Utility connected bidirectional soft-switching MFGCI . . . . . . . . . 41

2.12 Grid connected PV system with cascaded multilevel converter . . . . . . . . 47

2.13 Summary of control strategies for Photovoltaic applications _ . . . . . . . 48

2.14 The general layout of a Model Predictive Control [4] . . . . . . . . . . . 50 
2.15 The general layout of a Repetitive Controller [5] . . . . . . . . . . . . . 51

2.16 Typical configuration of synchronous rotating $(d q)$ reference frame control method $[6] \ldots \ldots \ldots \ldots \ldots$

2.17 Typical configuration of synchronous rotating $(d q)$ reference frame control method $[6] \ldots \ldots \ldots \ldots \ldots$

3.1 Annual Electric Demand of a Neighborhood in Miami. . . . . . . . . . . . . 67

3.2 Hourly Solar Irradiation. . . . . . . . . . . . . . . . . . . . 67

3.3 Hourly wind speed data. . . . . . . . . . . . . . . . . . 68

3.4 Simulated Model used in Matlab Simulink and HOMER software. . . . . . . 71

3.5 Average Electric Power Production for Wind Turbine System. . . . . . . . . 72

3.6 Average Electric Power Production for Hybrid Wind Turbine and PV System. 72

3.7 Comparison of power generated by wind turbine in A) Matlab Simulink and B) HOMER. . . . . . . . . . . . . . . . . . . . . . 73

3.8 Bus B2 voltage, active and reactive power, $\mathrm{DC}$ bus voltage of $\mathrm{AC} / \mathrm{DC} / \mathrm{AC}$ converter and wind speed pattern. . . . . . . . . . . . . . 74

3.9 Comparison of power generated by Photovoltaic Panels in A) Matlab Simulink and B) HOMER. . . . . . . . . . . . . . . . . . . . . 74

4.1 Block diagram of the configuration of the proposed system. . . . . . . . . 79

4.2 Hourly solar irradiation for one year. . . . . . . . . . . . . . 80

4.3 The equivalent circuit of a photovoltaic array. . . . . . . . . . . . 81

$4.4 \mathrm{I}-\mathrm{V}$ and $\mathrm{P}-\mathrm{V}$ curves of single module at $25^{\circ} \mathrm{C}$ for different irradiance . . . . 83

4.5 Irradiance, output voltage, output current duty cycle and generated power of PV system for the first 42 weeks of the year (Training Data) . . . . . 85

4.6 Irradiance, output voltage, output current duty cycle and generated power of PV system for the last 10 weeks of the year (Testing Data). . . . . . . . 85

4.7 Results of estimated duty cycle values compared to actual values. . . . . . . 86 
5.1 PV Plant Components: a) 4460 PV modules of three different types but each with rated power around $315 \mathrm{~W}, \mathrm{~b}$ ) Transformer, Energy meter, and Main dosconnect at the Point of Common Coupling(PCC), c) Smart field inverters, d) Revolution Wireless Power Quality Recorder, e) AC disconnect box which is connected to the AC output of the inverter, f) DAS that measures the multivariate time-series data from inverters, meter and weather station and securely stores in a cloud server. . . . . . . . . . 89

5.2 Histogram of real power ramp rates for Mar 1-10, 2017 . . . . . . . . . . . 94

5.3 Power ramping scenarios for the PV power plant. a)High power density ramp rate profile, b) High energy density ramp rate profile. . . . . . . . . 95

5.4 Current THD variation by Time and PV Power Production. (Data imported from Provision Power Quality meter) . . . . . . . . . . . . . 96

5.5 Voltage THD variation by Time and PV Power Production. (Data imported from Provision Power Quality meter) . . . . . . . . . . . . . . 97

5.6 THDs monitored over 1 month for the case study. a) Voltage THD for the PV power plant, b)Current THD for the PV power plant. . . . . . . . . 98

5.7 Current THD with respect to real power and time. . . . . . . . . . . 99

5.8 Voltage variation by Time and PV Power Production (Data imported from Provision Power Quality meter). . . . . . . . . . . . . . . 101

5.9 ITIC (CBEMA Curve) of Photovoltaic power plant at FIU. . . . . . . . . . . 102

5.10 Photovoltaic Power Plant Voltage and Current at the PCC. . . . . . . . . . . 102

5.11 Maximum RMS values for voltage, Power output, and Load . . . . . . . . . 103

5.12 Mapping scenario classes for use-case generation . . . . . . . . . . . 103

5.13 Voltage deviation study for PDL scenario. a)Existing Penetration, b) $60 \%$ Penetration . . . . . . . . . . . . . . 104

5.14 Voltage deviation study for MDL scenario. a) Existing Penetration, b) $60 \%$ Penetration. .................... 105

5.15 Feeder Losses for MDL and PDL Scenarios . . . . . . . . . . . . . . . 106

5.16 Irradiance and Load models for System Modeling. Irradiance model for a sunny day in October 2016, Irradiance model for cloudy day in October 2016, Load Model . . . . . . . . . . . . . . . . . . . . . 107

5.17 Maximum Feeder Voltage for different control modes on sunny day a)Power Factor $=0.85 \mathrm{~b}$ ) Volt/VAr mode . . . . . . . . . . . 108 
5.18 Maximum Feeder Voltage for different control modes on cloudy day a) Power Factor $=0.85 \mathrm{~b}$ ) Volt $/$ VAr mode . . . . . . . . . . . . . 109

6.1 Schematic diagram of the $L L C L$ filter. . . . . . . . . . . . . . . . . . . 115

6.2 Main harmonic current power density spectrum of inverter using unipolar modulation. . . . . . . . . . . . . . . 117

6.3 Proposed $L(L C L)_{2}$ system. . . . . . . . . . . . . . . . . . . . . 118

6.4 Bode plots of transfer functions $i_{g}(s) / v_{i}(s) \ldots \ldots \ldots$. . . . . . 120

6.5 Bode plots of transfer functions $i_{i}(s) / v_{i}(s) \ldots \ldots \ldots \ldots$

6.6 Size of the impedance of the $L L C L$ and the $L(L C L)_{2}$ filters for different frequencies. . . . . . . . . . . . . . . 121

6.7 Voltage and the current output waveform of typical single-phase full-bridge inverters. . . . . . . . . . . . . . . 121

6.8 Flowchart of the parameter design procedure of $L(L C L)_{2}$ filter. . . . . . . . . 125

6.9 Block diagram of the current control system. . . . . . . . . . . . . . 131

6.10 Root locus of the closed-loop transfer function of the system $\left(H_{s}\right)$ as (a) $L_{11}$ varies from $1.39 \mathrm{mH}$ to $2.52 \mathrm{mH}$ (b) $L_{22}$ and $L_{32}$ vary from $0.84 \mathrm{mH}$ to $1.485 \mathrm{mH}$ (c) $C_{f 1}$ varies from $0.5 \mu \mathrm{F}$ to $3 \mu \mathrm{F}$ (d) $C_{f 2}$ varies from $0.5 \mu \mathrm{F}$ to $3 \mu \mathrm{F}$ (e) $R_{f 1}$ varies from $0.08 \Omega$ to $0.39 \Omega$. (f) $R_{f 2}$ varies from $0.16 \Omega$ to

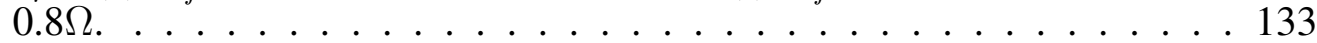

6.11 LLCL filter. (a) Grid voltage and grid-side current waveforms. (b) Power density spectrum of grid-side current (Experimental setup). (c) Power density spectrum of grid-side current (Simulation) . . . . . . . . 13

$6.12 L(L C L)_{2}$ filter. (a) Grid voltage and grid-side current waveforms. (b) Power density spectrum of grid-side current (Experimental setup). (c) Power density spectrum of grid-side current (Simulation) . . . . . . . . . . 136

6.13 (a) $L_{f} C_{f}$ current of $L L C L$ filter . (b) Power spectral density of $L_{f} C_{f}$ current in $L L C L$ filter (Experimental setup). (b) Power spectral density of $L_{f} C_{f}$ current in $L L C L$ filter (Simulation). . . . . . . . . . . . . . .

6.14 (a) Current of switching frequency trap $L_{f 1} C_{f 1}$ of $L(L C L)_{2}$ filter. (b) Power spectral density of $L_{f 1} C_{f 1}$ current in $L(L C L)_{2}$ filter (Experimental setup). (c) Power spectral density of $L_{f 1} C_{f 1}$ current in $L(L C L)_{2}$ filter (Simula-

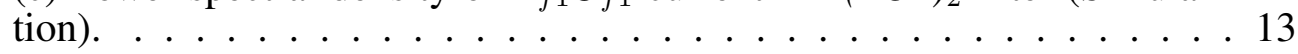

6.15 (a) Current of the double of switching frequency trap $L_{f 2} C_{f 2}$ of $L(L C L)_{2}$ filter . (b) Power spectral density of $L_{f 2} C_{f 2}$ current in $L(L C L)_{2}$ filter (Experimental setup). (c) Power spectral density of $L_{f 2} C_{f 2}$ current in $L(L C L)_{2}$ filter (Simulation). . . . . . . . . . . . . . . . . . . 139 
6.16 Bode plots of transfer function $i_{g}(s) / v_{i}(s)$ under different filter inductances. $\quad 140$

6.17 DC Link voltage when a 0.5 pu load is switched-in on $t=0.15$. . . . . . . 140

6.18 Experimental Setup . . . . . . . . . . . . . . . 141

7.1 Future grid that will be dominated by power electronic inverters (left) and traditional grid dominated by synchronous machines (right) . . . . . . 155

7.2 Basic idea of the Virtual Inertia Emulator (VIE) [7] . . . . . . . . . . . . 158

7.3 The VSG topology by VSYNC that uses the PLL in order to imitate the behavior of synchronous generator . . . . . . . . . . . . 161

7.4 structure of the VSG by using PLL, the detailed framework (top), and the block of the reference current(below). . . . . . . . . . . . 163

7.5 The VSG structure based on currentvoltage (voltagecurrent) model of SG:

(a) VSG topology, (b) currentvoltage model of SG. . . . . . . . . . . . 164

7.6 The VSG structure proposed by ISE Lab. . . . . . . . . . . . . . . . . 166

7.7 The VSG block diagram proposed by Kawasaki Heavy Industries(KHI). . . . 167

7.8 Block diagram of the system dynamics plus load damping . . . . . . . . 172

7.9 Block diagram of the system dynamics plus load damping . . . . . . . . 173

7.10 Block diagram of simple non-reheat steam turbine . . . . . . . . . . . 174

7.11 Governor steady-state speed characteristics. . . . . . . . . . . . . 174

7.12 Block diagram of speed governing system for steam turbine. . . . . . . . 175

7.13 Load Frequency control block diagram of an isolated power system. . . . . . 176

7.14 Schematic diagram of the VIE model. . . . . . . . . . . . . 176

7.15 Equivalent circuit of supercapacitor: (a) first order model,(b) third order model by implementing a voltage dependent branch. . . . . . . . . . . 180

7.16 Models for supercapacitor bank with a sixth order equivalent circuit. . . . . . 181

7.17 Control system designed for a HESS. . . . . . . . . . . . . . . . . . . . 184

7.18 The battery bank controller block diagram. . . . . . . . . . . . . . 185

7.19 The SC bank controller block diagram. . . . . . . . . . . . . . . . 186

7.20 An overview of the proposed system a)Hybrid Energy Storage Controller

b) Virtual Energy Inertia Controller . . . . . . . . . . . . 188 
7.21 The output power of photovoltaic arrays . . . . . . . . . . . . . . . . . 189

7.22 Battery and Supercapacitor power output. . . . . . . . . . . . . . . 190

7.23 SOC of the battery banks and Supercapacitor bank during intermittencies. . . 192

7.24 DC link voltage variation by solar intermittency. . . . . . . . . . . . 193

7.25 Total power output of Hybrid Energy Storage System (HESS). . . . . . . . 193

7.26 Variation of Frequency at POC while a big load connects at $\mathrm{t}=1 \mathrm{~s}$ and disconnects at $\mathrm{t}=2.5 \mathrm{~s} . \ldots \ldots \ldots \ldots \ldots$

7.27 Variation of IdIq references while a big load changes at $\mathrm{t}=1 \mathrm{~s} . \quad \ldots \ldots$

7.28 Active and Reactive power variation during pulsed load event. . . . . . 195

7.29 Voltage variation when a puled load is switched in. . . . . . . . . . 196 


\section{LIST OF ACRONYMS}

FSIG

PMSG

DFIG

PCC

LVRT

GA

ADP

HDP

VSI

CSI

PPWM

IGBT

THD

MOP

DVR

MPPT

PCS

DOE

NERC

HILF
Fixed-speed induction generator

Permanent magnet synchronous generator

doubly fed induction generator

point of common coupling

Low voltage ride-through

Genetic algorithm

Adaptive dynamic programming

Heuristic dynamic programming

Voltage source inverter

Current source inverter

Phasor pulse-width-modulated (PPWM)

Isolated Gate Bipolar Transistor

Total Harmonic Distortion

Multi-objective optimization problem

Dynamic voltage restore

Maximum power point tracking

Power conditioning system

Department of energy

North american electric reliability corporation

High-impact, low-frequency 


\section{CHAPTER 1}

\section{INTRODUCTION}

This introductory chapter contains five sections. The first section describes the background of the problem. The second section introduces motivations and research purposes of the dissertation. The third section articulates objectives and contributions of this research. The summary of the literature search of the problem is presented in the fourth section. Finally, the fifth section presents the general organization of this dissertation.

\subsection{General Statement of Problem Area}

The proliferation of photovoltaic (PV) power generation systems in the distribution power grid motivate utilities to monitor the power quality (PQ) effects of these renewable power generators in the electrical networks. The power quality may cause voltage and frequency fluctuations, in addition to harmonic emissions from low frequency to high frequency $[8,9]$. Nowadays most companies rely on sophisticated equipments to be able to compete with other competitors. This equipment could be affected by some power quality issues which are undetectable by the naked eyes. The benefits of high power quality of the power system are increasing the reliability of the systems, provide early prediction of incipient problems, decreasing the energy expenditure, preventing power outages due to hidden disturbances, assurance of data integrity and reducing corrupt data in smart grids, etc. It is very difficult to quantify the power quality concerns since they are different from the interaction between susceptible equipment and power quality. When "good" power quality for one equipment could be "bad" for another one [10]. Therefore, the best assessment criteria are standards. In 1982, IEEE standard 519 was first released and then updated in 2014. It sets some rules and steady-state limitations on Total Harmonic Distortion (THD) with reference to both current and voltage at the Point of Common

Coupling (PCC). However, exceeding these boundaries may be encountered by transient 
conditions. IEEE Standard 1547 was published in 2003 and the latest amendment was issued in 2014. This standard contains power quality, response to unusual situations, and installation assessment. The specified requirements are essential for interconnection of distributed resources (DR), including power inverters/converters and synchronous generators. These standards are valid to all DR technologies, with a combined capacity of 10 MVA or less at the PCC, connected to secondary and/or primary distribution voltages. The power quality concept has always been oversimplified and misunderstood, but due to a significant increase in the use of sensitive electronic devices and sophisticated data management devices, the assessment of power quality has become an inevitable task.

A typical schematic of a $180 \mathrm{~kW}$ photovoltaic system for industrial locations is shown in Fig. 1.1. In this configuration, diesel generators are used as backup power. However, generator back-up power is the only size to meet the sensitive loads. Please be advised that this configuration is not the layout of the Photovoltaic power plant at Florida International University and it is just an example.

In order to limit the excessive current harmonics, which are mostly produced by sine pulse width modulation (PWM), a low-pass power filter is usually put in between a voltage-source inverter (VSI) and the grid $[11,12]$. The use of the PWM system necessitates an output filter to limit the grid-injected current harmonics, in order to satisfy the IEEE 1547.2-2008 and IEEE 519-2014 standards. L-filters are typically used but they have the drawbacks of slow dynamic response and big inductor value [13,14]. The firstorder $L$ filter can meet the standards for the grid interconnection with notably smaller size and cost compared to an $L C L$ filter, primarily for applications in several kilowatts [15-17].

For industrial uses, the cost of the components is a vital aspect of selecting the power filter for the grid-tied inverter. Owing to the growing cost of copper, various methods have been implemented to cut down the price of the power filter. One helpful way is to 


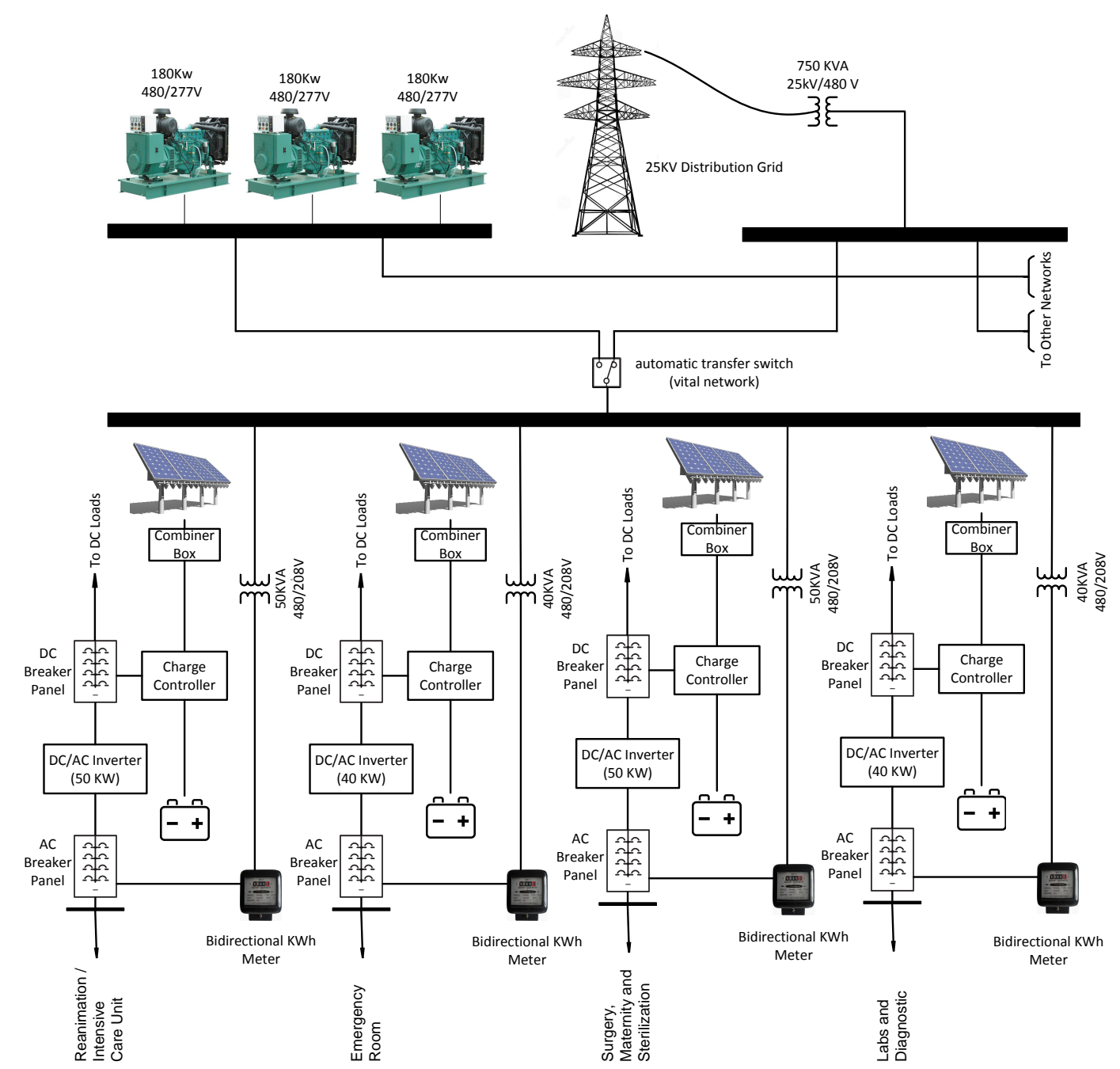

Figure 1.1: Typical schematic of a $180 \mathrm{~kW}$ photovoltaic system for industrial locations.

increase the switching frequency of the inverter where the method, surely, depends on the device proficiency and cost.

On the other hand, large influences of weather events on electric power systems, especially when dominated by renewable generation, and following disturbances have caused the rising worldwide need for addressing the concern of resiliency in power system. Resiliency is the capability of power systems to survive high-impact but low-probability events in an effective way while guaranteeing the smallest probable interruption in power 
supply, and enabling a fast restoration and recovery to the regular operation state. The main purpose of introduction of Microgrids were initially addressing the appearance of high-utilization of DERs in distribution grids. However, they recognized as valuable replacements to central bulk generation in power systems planning and operation. Microgrids present distinctive opportunities in power systems such as higher power quality, reduction in greenhouse gases (GHG) emission, improved reliability, offering energy efficiency, employment of less costly renewable energy sources, and providing an efficient and quick response for furnishing loads in remote regions [18]. The essential utilization of power electronic devices such as inverters in Microgrids can significantly decrease the equivalent rotational inertia of the electric power grid. Consequently, the Microgrids integration to the electric can be restricted. In addition, low inertia may lead to the deterioration of resiliency of Microgrid that reduces its frequency-stability. In a traditional power system, swing equations show the resilient power system that functions with a lower ratio of change of frequency in the occurrence of power imbalance, therefore with a higher inertia. The power imbalance mostly happens because of large active and reactive load variations, momentary faults, and instants of integration Microgrids for exports and import [19].

The inertial property in power system prevents the load shedding procedures, by dedicating that time for compensating control schemes to regulate generation to the varying situation. As the renewable-based distributed generation components begin to increase their footprint on the electric power networks, inertia in the Microgrid has become an interesting topic. Generally, the main sources of inertia in the power system are the classic steam turbines (typically synchronous generators) and the big motors in industrial units. Conversely, the renewable electric generators such as PV cells, being connected into the grid by application of the inverters that could not supply any inertia to the system. As a result, in the Microgrids that are connected to a weak grid or are not grid connected, 
there is either very small or no inertial response, that might cause terrible impacts to the Microgrid system [20].

\subsection{Literature Review}

As an environmental friendly and renewable energy source, solar generation has recently observed faster propagation throughout the world. However, as a result of the stochastic nature of solar irradiation, the subsequent fluctuations in solar energy significantly handicap large-scale integration of PV into distribution power grids. Reduction of inertial response which is a consequence of incompatibilities amongst the power demand and generation in the (micro) grid is another technical challenge of adding high levels of PV generation in the electric grid. Because of increasing the instantaneous power or loads with large startup current, energy management and power control of a system with low rotational inertia is a vital concern. Such a high current in a short time not only requires greater rating of the power devices, but also can probably cause the voltage and frequency of the system to drop in the entire Microgrid [21].

Historically, the power grid, especially the distribution system, has been designed and optimized for power flow in one direction: from central generators to substations through the transmission network, and then through feeders to individual consumers. As penetration of distributed solar generators, such as rooftop PV solar panels, increases, it is envisioned that during some hours of the day, the power generated by the solar installation can exceed power consumption needs, and therefore, power flow will be in the reverse direction, from individual consumers through the feeders to the substation and possibly beyond, into the bulk power system. This can be especially true for residential customers who are not at home during the workday. 


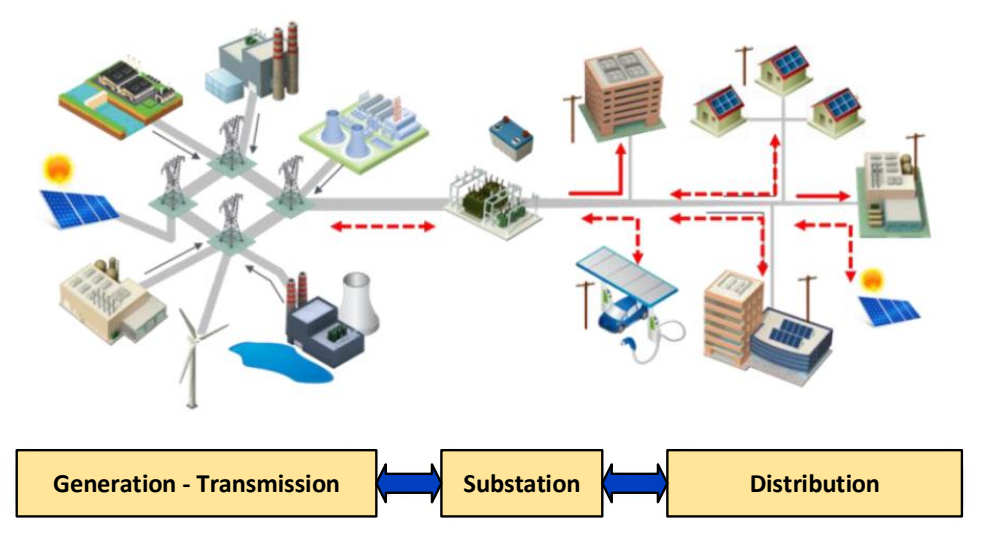

Figure 1.2: Two-way coupled Transmission and Distribution grid

To protect the network from threats related to PV interconnection, utilities and other electric energy entities imposed rigorous technical requirements and grid code regulations which are classified into four major categories: (i) fault ride through requirements, (ii) active and reactive power responses following disturbances, (iii) active power control or frequency regulation support and (iv) reactive power control or voltage regulation capability [7]. In this effort, the advanced, algorithmic and consolidated technical guidelines for specification of large scale PV grid interconnection scheme, reactive power capability and power control requirements for interconnection of variable generating plants to the power system are proposed. Also smart inverter based system in laboratory scale which follows UL 1741 IEEE 1547, 1547.a, 1547.1 and IEC 61850 and IEEE 2030.5 standards will be implemented.

Another challenge of using power conditioning units in PV system are harmonics. A low-pass power filter is often put in between a voltage-source inverter (VSI) and the grid to limit the excessive current harmonics, which are mostly produced by the sine pulse width modulation (PWM), to inject into the point of grid connection [22]. In comparison to a first-order L filter, an LCL filter can satisfy the standards for grid interconnection with notably smaller size and cost, primarily for applications in several kilowatts. Also LCL 
or higher order filter is needed to attenuate switching frequency harmonics too meet grid codes. Owing to the growing cost of copper, various methods have been implemented to cut down the price of the power filter. One helpful way is to increase the switching frequency of the inverter where the method, surely, depends on the device proficiency and cost.

A possible solution for regulating the fluctuating output power of a PV plant is to integrate a hybrid energy storage system (HESS) that has both high energy density storage battery and high power density storage super capacitor [7].

More than that, delivering high power in a short period of time is destructive to batteries, but it is the challenge that supercapacitor can best mitigate. In peak power situations, the super capacitor is capable of delivering or receiving energy, therefore it can act as a load-flattening device for the battery. If this is done, the battery output power would become closer to the average load demand, hence decreasing its RMS and peak currents [23].

Another method to emulate virtual inertia with energy storage system (however in this research a HESS is implemented) is called the Virtual Synchronous Machine (VISMA), Virtual Synchronous Generator (VSG), or Virtual Inertia Emulator (VIE). It modify the DG units in a way that they operate like synchronous generators, exhibiting an amount of inertia and damping properties of conventional synchronous machines for short time intervals. Then a significant share of DGs/RESs in islanded microgrids can be maintained without compromising system stability by a basis from virtual inertia concept. A VIE consists of energy storage, inverter, and a control mechanism as shown in Figure and it is usually located between a DC bus and the grid. The VSG shows the DC source to the network as an SG in a viewpoint of inertia and damping property. Virtual inertia is emulated in the system by controlling the active power through the inverter in inverse proportion to the rotor speed [24]. Aside from higher frequency noise due to switching of 


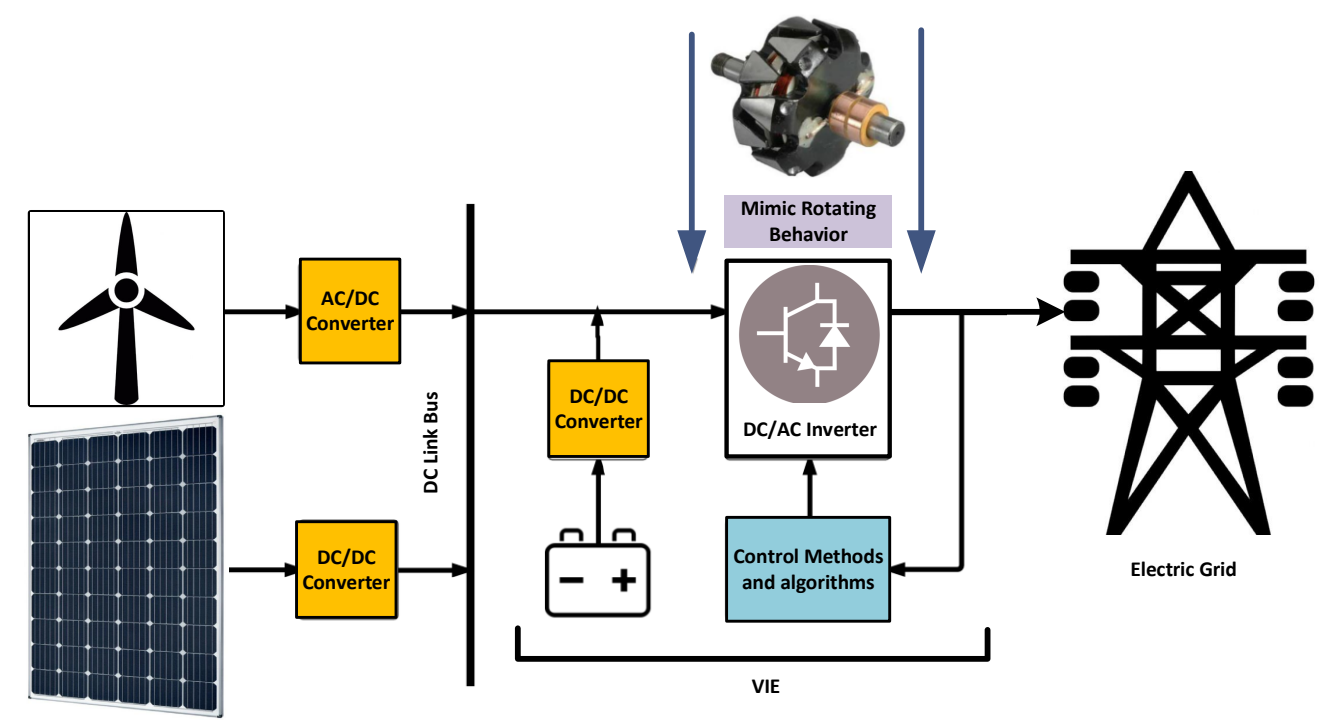

Figure 1.3: General configuration and concept of the VIE

inverters power transistors [25], there is no difference between the electrical appearance of an electro-mechanical SG and electronics VIE, from the grid point of view.

\subsection{Motivation and Purpose of Research}

As mentioned in the earlier parts and the literature reviews, power electronic devices are increasingly used within electrical systems for efficient control and use of electric energy. On the other hand, the renewable sources of energy are intermittent by nature. Combination of these two parameters, impose technical challenges into the electrical grid. These challenges can be due to changes in feeder voltage profiles, increase of harmonic injection into the grid, lack of resiliency when renewable penetration is high and finally frequent operation of voltage regulation devices such as load tap changers (LTCs), line voltage regulators (VRs), and capacitor banks (CBs). There are two critical components in a grid connected photovoltaic system, which have the most impact on the power output quality and system performance under supply (or load) disturbance. First is the low pass 
filter, which is commonly placed at the output side of the inverter. Second is the dc bus that needs to maintain a constant voltage in order to ensure constant and smooth power flow to the inverter.

First part of the research that has been done in this dissertation focuses on the impact of large PV penetration in the distribution system. A further motivation is that the nature of the impact of PV in the distribution system differs from one area to another and depends on the geographic location of solar PV site and point of interconnection in the distribution grid. For this reason, studies performed for one area of the power system cannot in general be sufficient for all other areas. This will help local power utility to understand the interactions, models that are required to be developed, power quality impacts as a function of penetration level.

The second part of the research emphasis on reduction of the influence of the grid harmonic currents and voltages. Harmonic compensation is regularly implemented for a grid-tied inverter. However, because of the growing price of copper, many measures have been adopted to cut down the cost of the power filter. One effective way is to raise the switching frequency of the inverter where the solution certainly depends on the device modeling and costs. This study fill out this gap by designing a new low pass filter topology. Generally, there are two major sources of inertia in the power system, which are the synchronous generators and the industrial motors. However, the future grid with domination of the renewable resources that mostly are connected to the grid through a power electronic inverter, cannot provide required inertia to the system. Therefore, smart grids and power systems will have either very slight or no inertia property. This phenomenon could lead to severe problems in the grid like frequency variation and fluctuating power output. Therefore, providing virtual inertia and smoothing the power output is increasingly important. 


\subsection{Research Objectives and Original Contributions}

The objectives of this research are on the issues that are stated above. The main goal of this research is to understand the interactions, potential power quality issues in different penetration level, as well as providing advanced solutions for renewable energy integration. The nature of the impact of PV in the distribution system differs from one area to another. The uniqueness of this research is that not only it suggests advanced solutions by designing a new type of filter in a laboratory based prototype and virtual inertia, but also it does fill the gap of in-depth analysis of the impact of high PV penetration in a distribution grid. The thesis has completed the following major activities:

1. Analyzing the trends of voltage fluctuations, harmonics, and frequency of the photovoltaic power plant, identifying threshold issues affecting performance, and proposing solutions for maintaining or improving of distribution grid performance.

- Investigation of capacitor bank operations and voltage regulator operations due to increase renewable penetration.

The purpose of this research is to conduct detailed analysis of effects of medium scale PV plant connected on a distribution system. The evaluation of power quality criteria for FIU photovoltaic power plant and early detection of an incipient problem has been conducted. First, real data from Provision PQ meter and Also Energy data acquisition system, with 1 minute resolution, was gathered a categorized. Then a comprehensive analysis has been conducted to figure out the voltage, current THD, and voltage violations. Furthermore, this study provide improved methodologies that will allow local utility to select optimal control setting in order to reduce the 
number of switching operations quickly and accurately. As a result the life time of legacy device in presence of high PV penetration will increase.

2. Design and hardware laboratory prototype implementation of a new low-pass power filter named $L(L C L)_{2}$ filter to reduce the harmonics at the switching frequency and multiples

- Close-loop stability analysis of the current controller

- Efficiency comparison between the $L L C L$ and $L(L C L)_{2}$ filters

- Size estimation of $L L C L$ and $L(L C L)_{2}$ filters

- Small-signal model of the dc-link dynamics considering the instantaneous power for bi-directional ac/dc power flow applications

In this research, the principles of the conventional $L L C L$ filter and parameter design of the $L(L C L)_{2}$ filters were proposed. Since grid-side inductance of the $L L C L$ filter is mainly decided by the harmonic currents around double the switching frequency instead of those around the switching frequency, it was replaced by a small trap at double the switching frequency. Compared to the $L L C L$ filter, the replacement results in the reduction of the total inductance size, and hence, the total loss of the filter. The inverter-side inductance is divided into three parts to place resonant branches in between them. The proposed $L(L C L)_{2}$ filter has lower

loss and better performance at high-order harmonics attenuation. In the proposed design, the maximum power factor variation remained unchanged and the current THD improved by $7.77 \%$.

3. Design of a combinatorial advanced solution for PV integration to address intermittency and provide inertial response. This work has been done by implementing hybrid battery-super capacitor energy storage system and Virtual 
Inertia Emulator (VIE). Ramp rates are one of the measure of performance in intermittency mitigation. Drop in power output and frequency oscillation will be measure of inertial response of the system.

- Controller Design for the Grid connected inverter of the distributed generation System

- Controller Design for the DC/DC Converters of the hybrid energy storage system (HESS)

\section{- Power Smoothing of Short-Term Photovoltaic Power Fluctuations}

The operation of a photovoltaic (PV) generating system under intermittent solar radiation is a challenging task. Furthermore, with high penetration levels of photovoltaic energy sources being integrated into the current electric power grid, the performance of the conventional synchronous generators is being changed and grid inertial response is deteriorating. This research proposes a combined virtual inertia emulator (VIE) and a hybrid battery-super capacitor-based energy storage system for enhancing the inertial response and smoothing the short-term power fluctuations simultaneously. The proposed system overcome the slow response of battery system (including dynamics of battery, controller, and converter operation) by redirecting the power surges to the super capacitor system. Moreover, it enhance the inertial response by emulating the kinetic inertia of synchronous generator. Control systems for the VIE and battery-super capacitor storage system are presented in this research. Correspondingly, simulation results are discussed to validate the effectiveness of the proposed scheme. Matlab Simulink software has been considered to develop control designs of VIE and Hybrid Energy Storage System (HESS). The recommended method is capable of achieving voltage and frequency regulation and effective management of the hybrid storage system. Since the suggested technique 
focuses on short-term fluctuations and includes no long-term power regulation, it needs no mass storage device. Thus, the method is economical.

On top of the major activities, this thesis presents an analysis of technical and financial viability of hybrid grid/Renewable Energy System (RES) configurations for a neighborhood in Miami. Assessment criteria comprised reduction in net present cost (NPC), cost of energy (COE) and greenhouse gases (GHG). Moreover, an artificial neural network based duty cycle estimation for maximum power point tracking in photovoltaic system is studied. The proposed technique is implemented in Matlab/Simulink and compared with the conventional method of incremental conductance.

\subsection{Dissertation Organization}

The rest of this dissertation is organized as follows: Chapter 2 presents investigation of standards and threshold issues affecting performance of grid-connected photovoltaic systems. In this chapter a handy extensive investigation on grid connected photovoltaic system is conducted. Moreover, an analysis of the challenges of photovoltaic integration into the grid and some integration solutions are also presented. Standard requirements for PV integration, grid codes, and power electronic inverter topologies for Photovoltaic interconnection to the grid are studied.

Chapter 3 introduces analysis of carbon tax as an incentive toward building sustainable grid with renewable energy utilization. For this purpose, an evaluation of the Co2 tax level of the cost-effectiveness of the system is also conducted. Outcomes demonstrate that there is the remarkable potential of $\mathrm{Co} 2$ mitigation along with $\mathrm{COE}$ reduction and sustainable and resilient energy development from employing RES.

Chapter 4 discusses an artificial neural network based duty cycle estimation for maximum power point tracking in photovoltaic system. The proposed technique is imple- 
mented in Matlab/Simulink and compared with the conventional method of incremental conductance. Simulation results show a good performance of the ANN based MPPT controller.

Chapter 5 analyzes the trends of voltage fluctuations, harmonics, frequency, and legacy devices of the photovoltaic test-bed. This chapter presents monitoring and assessment of a 1.1MW photovoltaic power plant at Florida International University, Miami, Florida. The assessment criteria were the IEEE 519 and 1547. It concluded that at current level of photovoltaic penetration (16\%), Current THDs violates the 5\% limit value at the Point of Common Coupling (PCC). Moreover, except from a few short duration voltage impulses, no voltage violation has been observed.

Chapter 6 proposes a new topology of higher order power filter for single-phase gridtied voltage source inverters, named $L(L C L)_{2}$. A comparative study and discussions on the subject of the traditional $L L C L$ filter and the proposed $L(L C L)_{2}$ filter have been conducted and assessed through an experimental hardware implementation on a $700 \mathrm{~W}, 120 \mathrm{~V} / 60$ $\mathrm{Hz}$ single-phase grid-tied inverter. Furthermore, a straightforward engineering design benchmark is suggested to discover parameters of the $L(L C L)_{2}$ filter. Moreover, stability analysis, loss analysis and an optimization of the $L(L C L)_{2}$ filter parameters have been conducted in this study. The analysis shows that in comparison with the $L L C L$ filter, the $L(L C L)_{2}$ filter not only has lower voltage drop and less total inductor size, but also has improved performance in decreasing high order current harmonics.

Chapter 7 develops a combinatorial advanced solution for PV integration to address intermittency and provide inertial response. This chapter proposes a combined virtual inertia emulator (VIE) and a hybrid battery-supercapacitor-based energy storage system . VIE provides a method which is based on power devices (like inverters), which makes a compatible weak grid for integration of renewable generators of electricity. This method makes the power inverters behave more similar to synchronous machines. Consequently, 
the synchronous machine properties, which have described the attributes of the grid up to now, will remain active, although after integration of renewable energies. Through these studies, it demonstrated that the recommended method is capable of achieving voltage and frequency regulation and effective management of the hybrid storage system.

Chapter 8 summarizes the dissertation outcomes, concludes the significance of this research, discuss the results, and finally makes recommendations for the future works. 


\section{CHAPTER 2}

\section{INVESTIGATION OF STANDARDS AND THRESHOLD ISSUES AFFECTING PERFORMANCE OF GRID-CONNECTED PHOTOVOLTAIC SYSTEMS}

\subsection{Overview}

It has been very clear from recent studies and documentations the fossil fuels would last only a few more decades. The cost of fossil fuel has become a major challenge for all of human kind. Not only the economic value but the environmental impacts of fossil fuels have clearly made us move toward alternatives [26-28]. The greatest alternatives that can really make a difference for sustainability, such as reducing green-house gases and long term economics, are the renewable energy sources (RES) like wind and solar power. Solar photovoltaic (PV) industry is the dominant type of RES technology integrated to power grid systems as its cost reduces over the next ten years, while deployment of PV systems continues to increase quickly. As penetration of PV on the grid grows, finally reaching hundreds of gigawatt (GW) interconnected capacity, a diversity of methods require to be taken into account and also implemented at various scale, for reliable and cost-effective connection into the power grid [29].

Since many PV interconnection applications involve high penetration scenarios, the process needs to allow for a sufficiently rigorous technical evaluation to identify and address possible system impacts. Thus, except of reducing the PV cost installation, others issues such as standardization, simple improvements in design, better power electronics, and simplified procedures for grid integration are already improving the economics of PV systems. 


\subsection{Problem Statement}

They are many review studies on grid connected PV systems in the literature. The comparison of the most recent literature reviews are present in this part. In [30] authors studied the current trend of PV power plants development in the world, comparison of grid codes for fault ride through (FRT), voltage, frequency, active power, and reactive power was analyzed. After that, voltage stability, frequency stability, active power regulation, and reactive power regulation was studied. At last, the compliance technologies were investigated. Authors of [31] reviewed the technical requirements of PV systems with microinverters by analyzing the U.S. National Electrical Codes, standards and utility grid-interconnection application, Michigan state requirements, barriers and solutions for plug-and-play Photovoltaic systems, and advantages of microinverters. Ref. [32] studied the ratio between load and PV power, possible complications associated with high penetration PV into the grid, grid-connected inverters, and islanding detection methods. In [33] standards and specifications of grid-connected PV inverter, grid-connected PV inverter topologies, Transformers and types of interconnections, multilevel inverters, soft-switching inverters, and relative cost analysis have been presented. [34] did a review on prospects and challenges of grid connected PV systems in Brazil. [35] mostly focused on the techno- economic analysis of the grid connected PV system for building application. [36] reviewed the technical barriers of PV system development. The authors did a survey on categorizing the grid-connected and stand-alone PV systems, energy policy, a number of technologies implemented in PV cells, maximum power point tracking (MPPT), energy management, energy optimization, issues related to storage of energy in PV systems, hybrid PV systems, environmental and economic concerns, operation and maintenance issues. Ref. [37] provided analysis, explanation, and introduction on typical distributed MPPT and centralized MPPT. In [38] guidelines and standards of 
the grid connected PV generation systems, effects of large PV integration into the power grid, power quality requirements, protection methods, and control capabilities have been investigated. As it can be seen each study mostly focus on only limited aspects of PV technical specification, and there is no comprehensive review on this topic.

\subsection{Solution Approach}

In this chapter a handy extensive investigation on grid connected photovoltaic system is conducted. The outline of the rest of this chapter is as follows. Section II analyses the challenges of photovoltaic integration into the grid. Some integration solutions are also presented in this section. In Section III the standard requirements for PV integration are studied. Grid codes are studied in Section IV. Section V analyses the power electronic inverter topologies for Photovoltaic interconnection to the grid. Most popular three phase inverters are investigated in Section VI. Most implemented control algorithms for PV systems are presented at Section VII. Some storage systems for PV applications are studied in Section VIII. Finally, the chapter is concluded in Section IX.

\subsection{Challenges to Integrate Solar Photovoltaic}

In spite of all advantages of PV, it might make some potential adverse effect on the present power grid. Solar is known as non-dispatchable resources. There is no control over the input these kinds of energy resources for later use when desirable [39]. The lack of control over the input has a direct relationship with unpredictability of the output power injected to the grid [40]. The incapability of generating on-demand power triggers stability and reliability concerns to the power system $[39,41,42]$. Some of the challenges that come along with using renewable energies are depicted in Fig. 2.1 [43]. 


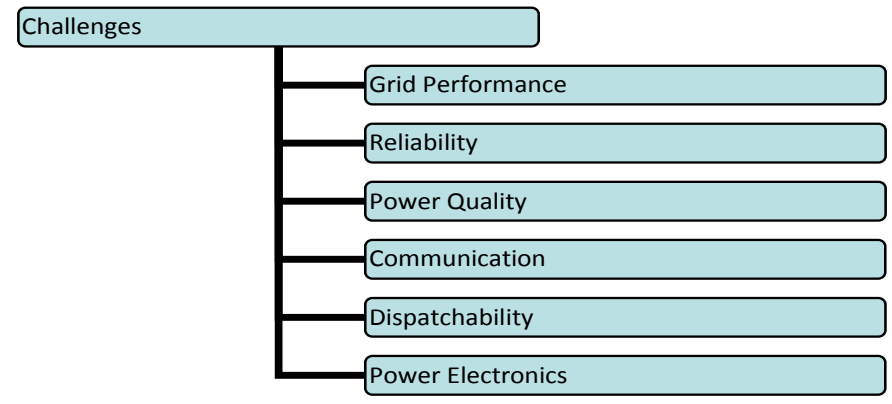

Figure 2.1: Challenges that arise when integrating renewable resources into the smart grid.

The information collected from an extensive survey on literature shows that the PV output power fluctuation due to solar irradiance intermittency is the most important problem of PV grid integration. Thus, large scale integration of photovoltaic system into the distribution grid introduces corresponding problems such as voltage regulation problem, harmonics, reactive power compensation, synchronization, energy storage, forecasting and scheduling, and load demand management systems. A classification of technical challenges of large-scale PV in the distribution systems are presented in Table 2.1.

Distributed system protection coordination in a feeder with high PV integration using widespread distributed feeder measurement and utilizing OpenDSS has been studied by [44]. Short circuit detection technique for the PV inverter by valuating the magnitude and slope $(\mathrm{d} / \mathrm{dt})$ of the PV inverter current is introduced in [45]. In order to prevent any contrary effects of the short circuit current, the proposed system either disconnects the inverter or transfers the inverter to a PV dynamic reactive power compensator (STATCOM).

The success of alternative energy is dependent upon the engineering equipment and infrastructure which it is based upon and its ability to capture and convert this energy [40]. The availability of solar power is dependent upon the position of the sun, angle at which the sun-rays fall upon the surface of the earth, and cloud location [43]. The places at which these renewable resources are available are typically far from their intended population areas. This would require extensive investment in transmission infrastructure 
Table 2.1: Technical Challenges in Regards to Implementation of Renewable Energy to the Smart Grid

\begin{tabular}{|l|l|}
\hline Technical Challenge & $\begin{array}{l}\text { Description of challenge and how it effects the smart grid } \\
\text { when renewable energy is integrated. }\end{array}$ \\
\hline \hline $\begin{array}{l}\text { Voltage Fluctuation / In- } \\
\text { termittency }\end{array}$ & $\begin{array}{l}\text { Major issue due to the intermittency of these renewable resources. } \\
\text { This is seen to occur because of the variance of available solar } \\
\text { energy at any given point throughout the day. Fluctuating voltage } \\
\text { can disturb sensitive equipment and possibly reduce the life of } \\
\text { power electronic devices. }\end{array}$ \\
\hline Harmonic Distortion & $\begin{array}{l}\text { Voltage distortion and fluctuation issues can be produced by in- } \\
\text { jected harmonics onto the grid. Power electronic devices and op- } \\
\text { erative non-linear appliances are the main sources of high per- } \\
\text { centage of total harmonic distortion (THD). }\end{array}$ \\
\hline Reactive Power Compen- & $\begin{array}{l}\text { Because of variations in the active and reactive power, a fixed } \\
\text { capacitor or switched capacitor or static compensator can be im- } \\
\text { plemented as a power regulator. }\end{array}$ \\
\hline Synchronization & $\begin{array}{l}\text { In order to ensure power quality in the grid, synchronization of } \\
\text { grid frequency, voltage, and phase is a crucial aspect. }\end{array}$ \\
\hline Energy Storage & $\begin{array}{l}\text { This is another aspect that is imperative with the purpose of ensur- } \\
\text { ing the reliability of power delivery. Energy storage systems are } \\
\text { being used to bring the instability and uncertainty under control } \\
\text { in the production of varying types of renewable energies. Some } \\
\text { existing energy storage methods are listed in the Energy Storage } \\
\text { section in this chapter. }\end{array}$ \\
\hline $\begin{array}{l}\text { Load Demand Manage- } \\
\text { ment Systems }\end{array}$ & $\begin{array}{l}\text { Knowing future weather patterns will play a crucial role in the } \\
\text { variability of renewable energy as it is introduced into the smart } \\
\text { grid. In order to reduce the intermittency on the network, accurate } \\
\text { forecasts must be produced to produce satisfactory power quality } \\
\text { and to perform viable load management systems. } \\
\text { quality improvement of the smart grid with renewable energy in- } \\
\text { tegration. With proper planning and management adequate power } \\
\text { quality can be supplied in a safe and efficient manner uniformly } \\
\text { across the entire power grid at varying loads and demands. }\end{array}$ \\
\hline Fonged
\end{tabular}


in order to insure the proper and secure transfer of energy produced. For the promise of alternative energy to be achieved, the following goals shown in Table 2.2 must be met [46].

Similarly, for the success of renewable energy, proper technology must be available for implementation [43]. Most researched technologies take anywhere between twenty to twenty-five years to demonstrate the feasibility and large-scale commercialization before they are implemented outside of the laboratory. The reason is that many of the processes for these technologies must be perfected and optimized for different operating environments. Apart from the optimization, all of the technologies must also be patented, tested, safety evaluations must be conducted, land procurement must be acquired, the financial analysis must be conducted, along with several other studies must take place before such technologies can be seen to commercial use.

Present Usage of PV Generation:Currently, it seems impossible that today's power grid could run on simple renewable resources unless there is a major advancement in energy conservation and improved energy efficiency.

One way to overcome this while using the available technology is to use other dispatchable renewable resources which can be kept running in reserve modes. Examples of some dispatchable renewable resources are [40]: Hydroelectric, Biomass, Geothermal.

Some of the other solutions that have been looked at is the solution of using compressed air storage, batteries, and the use of molten salts in appropriated solar thermal plants [47]. Some of the downsides from these approaches include losses in the process of energy storage, transfer and usage along with the limited density of energy that these systems are capable of storing with today's available technologies [48].

The inability to produce on-demand real and reactive power, in a way that generators have spinning reserve can be compensated by using energy storage systems. Therefore, the power generated by renewable energy resources like solar and the wind could be stored 
Table 2.2: Required Fundamentals for Grid Integration of Renewable Energies.

\begin{tabular}{|c|c|}
\hline Area of interest & Target Goals \\
\hline Standards & Must abide and comply with ANSI, UL, NEC, and OSHA standards for operational usage. \\
\hline $\begin{array}{l}\text { Implementation planning } \\
\text { and future forecasting }\end{array}$ & $\begin{array}{l}\text { - Must be able to perform for approximately } 25-30 \text { years. } \\
\text { - Modern energy management systems to incorporate variability of renewable re- } \\
\text { sources. } \\
\text { - Computational intelligent forecasting tools. } \\
\text { - Integrate storage elements } \\
\text { - Fleet management } \\
\text { - Closed loop control mechanism to reduce disturbances. }\end{array}$ \\
\hline $\begin{array}{l}\text { Performance of the com- } \\
\text { ponents }\end{array}$ & $\begin{array}{l}\text { Over a 25-30 year projected lifetime performance the device should be able to : } \\
\text { - Have less than } 5 \% \text { internal loss while in a fully charged. } \\
\text { - Have an efficiency of over } 90 \% \text {. } \\
\text { - Be able to perform over } 50,000 \text { cycles of charging and discharging with no less } \\
\text { than } 40 \% \text { of PV capacity within } 1 \text { minute. } \\
\text { - Must be supplied at a reasonable time frame and generate power on demand } \\
\text { regardless of the time of day. }\end{array}$ \\
\hline $\begin{array}{l}\text { Financials/Cost of overall } \\
\text { project }\end{array}$ & $\begin{array}{l}\text { - Must have feasible cost in regards to performance and return. } \\
\text { - Should cost less than } 14 \text { cents/ } \mathrm{kWh} \text { after the system is fully installed. } \\
\text { - Cost analysis studies should include cost for proposed solutions including the } \\
\text { cost of the PV plant, inverters, storage devices, hardwares, software, intercon- } \\
\text { nection, and other devices which will ensure proper and optimal operations of } \\
\text { the system. }\end{array}$ \\
\hline $\begin{array}{l}\text { Communication to and } \\
\text { from components on the } \\
\text { smart grid }\end{array}$ & $\begin{array}{l}\text { - Must allow monitoring and communication to and from the components within } \\
\text { each sub system. } \\
\text { - Safe to maintain and preventative against external attack. } \\
\text { - Must be able to be controlled and provide feedback to the supplying utility com- } \\
\text { pany. } \\
\text { - Communication protocols must be compatible with current power electronic de- } \\
\text { vices on the smart grid for having a homogeneously operating smart grid. } \\
\text { - Protocols used in communication cannot be replicated by other industries. } \\
\text { - Must be able to respond to electrical market prices and have capability to respond } \\
\text { to load forecasting in order to determine the optimal methods to provide the best } \\
\text { power quality within the smart grid. }\end{array}$ \\
\hline
\end{tabular}


and then later used in order to abide by the load balancing act and available energy [49]. In todays society, pumped hydro devices almost dominated large-scale energy storage system in the USA [50]. However, some battery energy storage System also known as (BESS) are installed [51].

Application of energy storage has been known for their ability to provide many of the auxiliary functions such as load leveling, peak shaving, voltage regulation, VAR support, frequency control, spinning reserve and power quality mitigation that the power system so desperately need [39]. Renewable resources on the grid can be used in order to fix problems with fluctuations of power by introducing storage of the produced energy and secondly with the concept of distributed generation [52].

\subsection{Grid Codes Requirements}

PV capacity reached a global total of $100 \mathrm{GW}$ as of 2012, establishing itself as just one of the expeditiously growing renewable resources. With the massive injection of power from renewable resources, into the energy grid; there is a definite need to keep power requirements uniform to ensure reliability and stability. The use of universal and detailed standards, that lay out specific guidelines for integrating renewable resources into the energy grid. These standards and so-called Grid codes have responsibilities such as: voltage and frequency stability, power regulation, response to atypical energy system conditions, and system restoration. Presented below are these topics from various national requirements.

\subsubsection{IEEE 1547}

The IEEE 1547 standards [1] were established to provide a national agreement on implementing distributed resources (DR) in the power system grid. IEEE Std 1547-2014 
Table 2.3: ANSI C84.1 Voltage Ranges for 120V Base.[1]

\begin{tabular}{|l|l|l|l|l|}
\cline { 2 - 5 } \multicolumn{1}{c|}{} & \multicolumn{2}{l|}{ Service Voltage } & \multicolumn{2}{l|}{ Utilization Voltage } \\
\cline { 2 - 5 } \multicolumn{1}{c|}{} & Minimum & Maximum & Minimum & Maximum \\
\hline Range A & $114(-5 \%)$ & $126(+5 \%)$ & $110(-8.3 \%)$ & $125(+4.2 \%)$ \\
\hline Range B & $110(-8.3 \%)$ & $127(+5.8 \%)$ & $106(-11.7 \%)$ & $127(+5.8 \%)$ \\
\hline
\end{tabular}

presents obligatory prerequisites for the interconnection of DR with the electric power networks. The focus of this standard is mostly on radial distributed feeder interconnections. For DR interconnected on the distribution grid, all parts of IEEE Std 1547-2014 needs to be fulfilled.

Standard IEEE 1547 establishes foundational criteria for all types of interconnected distributed energy resources (DER) connected to Area Electric Power Systems (EPS). Generally this standard is applicable up to $10 \mathrm{MW}$ of distributed generation, and sets mandatory requirements. The standard's main objectives are: technical requirements for interconnection and testing the interconnection, to EPS. The following sections will outline certain aspects of IEEE 1547.

Normally distribution voltage regulation occurs at substation level. However, According to IEEE 1547, photovoltaic and wind turbine systems-distributed energy resources (DER), as they are commonly referred to should not cause area electric power systems (EPS) to set the voltage at the point of common coupling (PCC) actively. In addition, the DER should not force the service voltage of Area Electric Power systems outside of operating ranges specified by ANSI C84.1-1995 (Range A). ANSI C84.1-1995 voltage ranges are shown in Table 2.3

Area EPS are designed to have radial one-way power flow, which is from the substation to the load. This brings a unique challenge because DER possibly will cause two-way power flow, which could affect the EPS voltage. For example, if generated power by a DER source is injected into the power system, the load current will be offset and reduce the voltage drop at the Area EPS; because of DER, Local EPS loads can be offset and 
Table 2.4: Interconnection system response to abnormal Voltages [1]

\begin{tabular}{|c|c|}
\hline Voltage range (\% of base voltage) $^{1}$ & Clearing time $^{(\mathbf{s e c})^{2}}{ }^{2}$ \\
\hline $\mathrm{V}<50$ & 0.16 \\
\hline $50 \mathrm{~V}<88$ & 2.00 \\
\hline $110<\mathrm{V}<120$ & 1.00 \\
\hline $\mathrm{V} 120$ & 0.16 \\
\hline
\end{tabular}

possibly affect the voltage. In addition, DER sources absorb (Inductive) and supply (Capacitive) reactive power into the system. These situations accentuate the need for the IEEE 1547 requirement that DER should not actively regulate voltage.

\subsubsection{Voltage}

There are system response requirements for typical voltages. When a voltage within the specified range given in table 2.4 is detected, the DER should discontinue energizing the Area EPS, in the period of the indicated clearing time. The time between the start of the abnormal condition and the discontinuing the DER energization of the Area EPS is called clearing time.

\subsubsection{Frequency}

When the power system frequency is detected within the ranges listed in Table 2.5, the DER should discontinue energizing the Area EPS before clearing time limit. The Clearing time is the period of time between the beginning of the condition and the DER discontinuing to energize the Area EPS. If there is an Area EPS disturbance, the DER is not allowed to connect back to the power system until Area EPS voltage is restored to the specifications listed in ANSI C84.1 - 1995 (Range B) and frequency within: $59.3 \mathrm{~Hz}$

\footnotetext{
${ }^{1}$ Base voltages are stated in ANSI C84.1 - 1995 as the nominal system voltages

${ }^{2}$ maximum clearing times for $\mathrm{DR} \leqslant 30 \mathrm{~kW}$ default clearing times for DR $>30 \mathrm{~kW}$
} 
Table 2.5: Interconnection system response to abnormal Frequencies [1]

\begin{tabular}{|c|c|c|}
\hline DER size & Frequency,range $(\mathrm{Hz})$ & Clearing,time (sec) \\
\hline \multirow{3}{*}{$\leqslant 30 \mathrm{~kW}$} & $>60.5$ & 0.16 \\
\cline { 2 - 3 } & $<59.3$ & 0.16 \\
\cline { 2 - 3 } & $<\{59.857 .0\}$ & Adjustable \\
& (adjustable set point) & 0.16 to 300 \\
\hline$>30 \mathrm{~kW}$ & $<57.0$ & 0.16 \\
\hline
\end{tabular}

Table 2.6: Maximum Harmonic Current Distortion in Percent of Current [1]

\begin{tabular}{|l|c|}
\hline Individual harmonic order h (odd harmonics) & Percent (\%) \\
\hline \hline $\mathrm{h}<11$ & 4.0 \\
\hline $11<\mathrm{h}<17$ & 2.0 \\
\hline $17<\mathrm{h}<23$ & 1.5 \\
\hline $23<\mathrm{h}<35$ & 0.6 \\
\hline $35<\mathrm{h}$ & 0.3 \\
\hline Total demand distortion (TDD) up to the $\mathrm{h}=50$ harmonic & 5.0 \\
\hline
\end{tabular}

to $60.5 \mathrm{~Hz}$. Furthermore, the system should include a fixed or adjustable delay, up to a maximum time of five minutes, which ensures no reconnect until Area EPS steady-state frequency and voltage are returned to the aforementioned ranges in Table 2.5.

\subsubsection{Power Quality}

The dc current is not allowed to be bigger than $0.5 \%$ of the nominal output current at the PCC. Moreover, there are limitations for injected harmonic currents into the Area EPS at the point of DER connection; given in Table 2.6. The concern about dc current injection and dc voltage bias into the grid by PV system is studied in [53]. In this section a single-phase inverter for PV application with low-frequency transformer (LFT) investigated. Total harmonic distortion (THD) is another measure of power quality in OV systems. Current THD has bigger values in cloudy days, even there is no resonant in the network. 


\subsubsection{Islanding}

One of the most important concerns in utilizing PV in power system is islanding. Islanding happens when a line is disconnected and is energized by one (or multiple) DERs, whilst that section of the EPS is electrically cut off from the remainder of the EPS. If this situation is not discovered quickly, it may introduce dangerous safety condition [54]. During unintentional islanding, the DER shall have the means to recognize the accidental island and discontinue to energize the EPS in less than two seconds of the island's creation.

Other General requirements:

- IEEE 1547-4.1.3: The DER shall be in parallel with Area EPS and does not cause any voltage deviations at PCC larger than $\pm 5 \%$ of the nominal voltage of the Area EPS. Furthermore, unacceptable flicker shall not be produced by the DER for other customers on the Area EPS.

- IEEE 1547-4.1.5: the Area EPS shall not be energized by the DER while the Area EPS is not energized.

- IEEE 1547-4.1.7: An accessible, lockable, and clearly distinguishable disconnection device shall be provided to disconnect Area EPS from DER.

- IEEE 1547-4.1.8.2: The system of interconnection should be able to tolerate current and voltage swells in agreement to the conditions provided in IEEE standard C37.90.1-2012 or IEEE standard card C62.41.2-2002 as applicable.

- IEEE 1547- 4.1.8.3: The connected parallel DER to the system should be able to tolerate $220 \%$ of the interconnection system nominal voltage.

- IEEE 1547-4.2.1: The DR unit should stop energizing the Area EPS for faults on the Area EPS circuit to which it is connected. 
- IEEE 1547-4.2.2: The DER should halt energizing the Area EPS, earlier than reclosure by the Area EPS.

- IEEE 1547-4.2.2: The DR and its linked system should not inject dc current bigger than $0.5 \%$ of the nominal current at the PCC.

\subsection{Grid codes in Different Countries}

This section will serve to outline the main objectives of various national grid codes.

1) Germany: The nation of Germany retained its world rank in 2012 as the third largest investing country in renewable resources. $7.6 \mathrm{GW}$ of solar capacity was installed, making it the largest for any country. As such a detailed survey of Germany's Grid code is shown below. The Grid Code's objectives are keeping safety and reliability of network operation in accordance with the requirements of Energy Economy Law (ENergiewirtschaftsgesetz EnWG). The grid code allows for on-site calculation of minimum and maximum power at which the plant is connected to the medium voltage network. The grid code specifies that DER shall be dynamically grid supported. More expressly DER (PV system) must have the capability to remain connected during a fault, support or provide system with reactive power for the time of fault, and absorb similar or smaller reactive power after the cessation of a fault.

With respect to PV systems (specified as type 2 plants) in German grid code; they must not disconnect from the network when a voltage drop to $0 \%$ Uc and time duration of $\leqslant 150$ ms occurs. Fig 2.2 indicates ranges for disconnection, and ranges where DER must stay grid connected. If there is a voltage drop above borderline 1, the DER must remain stable and connected. Voltage drops above borderline 2, but below borderline 1, shall also be ridden through. Based upon network operation agreements certain options are available for this range: 


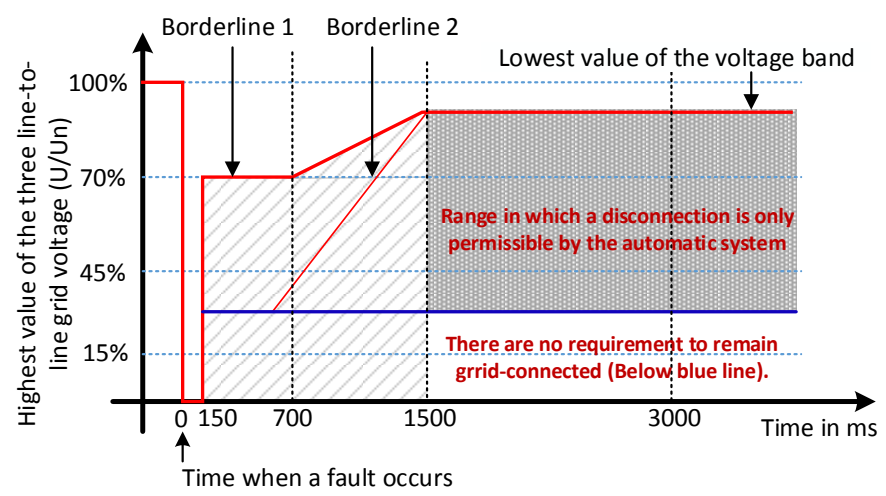

Figure 2.2: Fault Ride Through Germany

Below borderline 2, brief disconnection times, and possibly longer disconnection times are possible.

- Feed-in of short circuit current

- Based on the grid connection concept borderline 2 can be moved

- Brief disconnection times of up to 2 seconds.

In accordance with the grid active power control code's requirements, the power generation units should be capable of reducing power output. The subsequent criteria permit network operator to momentarily restrict injecting power, or cut-off the power generation plant:

- Dangerous system operations risks

- Congestion in the power network risks

- Unintentional islanding risks

- Dynamic or static grid instability risks

- Unstable conditions risks owing to frequency increase

- In order to perform repairs or construction 


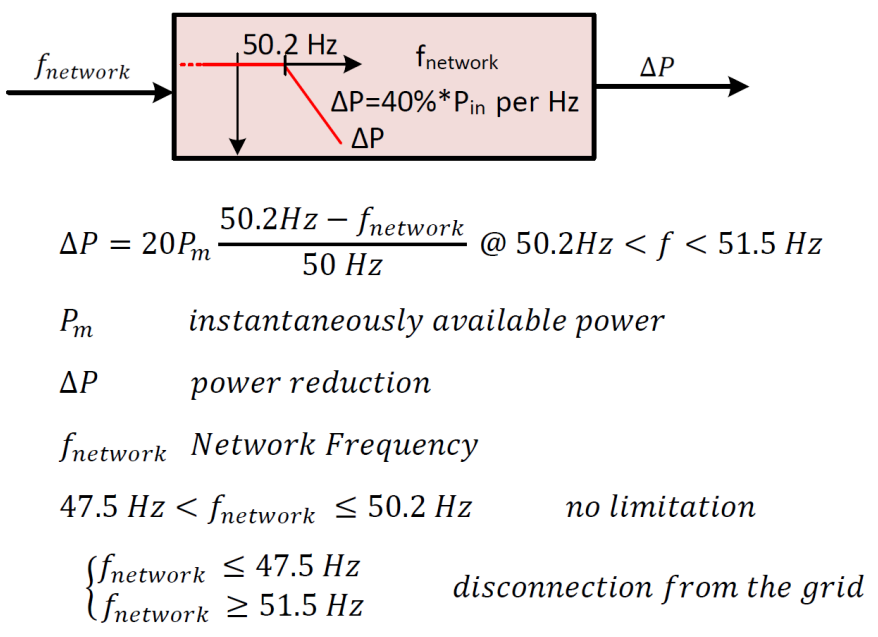

Figure 2.3: Dynamic response of Germanny's power grid.

- For feed-in, production, or network security management.

In addition there shall be a set point given by the network operator that must be attainable from any operation point. The generation plant must be able to reduce power in levels of $10 \%$ or smaller of the agreed upon rated output power.

As shown in the below Fig 2.3, when system frequency rises above $50.2 \mathrm{~Hz}$, there must be a reduction in output power. The power reduction shall be reduced with a gradient of $40 \%$ / Hz of instantaneously available power, and shall not be increased until frequency is below $50.05 \mathrm{~Hz}$. For frequencies greater than $51.5 \mathrm{~Hz}$ and less than $47.5 \mathrm{~Hz}$, the generation plant shall be disconnected from the grid.

Static grid support:Generating plants must have the capability to deliver reactive power at all operational points in line with the following displacement factor in the grid's connection point: $\cos (\varphi)=0.95_{\text {underexcited }}$ to $0.95_{\text {overexcited }}$

Reactive power will be delivered only for the duration of the feed-in operation. Therefore, there is no necessity to deliver reactive power at night time when no irradiance can be collected from the sun. Reactive powers set point can be fixed or variable signal by the network operator. Criteria for the value of the set point are: 
Table 2.7: LVRT requirement in China grid.

\begin{tabular}{|c|c|}
\hline Voltage Drop & During Time \\
\hline $20 \% \mathrm{UN}$ & $1 \mathrm{~s}$ \\
\hline $40 \% \mathrm{UN}$ & $1.57 \mathrm{~s}$ \\
\hline $60 \% \mathrm{UN}$ & $2.14 \mathrm{~s}$ \\
\hline $80 \% \mathrm{UN}$ & $2.71 \mathrm{~s}$ \\
\hline $90 \% \mathrm{UN}$ & $3 \mathrm{~s}$ \\
\hline Test should carried under three phase balanced and \\
unbalanced voltage drop
\end{tabular}

- Fixed displacement factor of $\cos (\varphi)$

- Or a variable displacement factor depending on active power $\cos (\varphi)$

- Variable reactive power depending on voltage $\mathrm{Q}(\mathrm{U})$

2) China: In 2011, Q /GDW 617-2011 "Technical rule for photovoltaic power station connected to Power Grid" and Q/GDW 618-2011 "Test Procedures for photovoltaic power station connected to Power Grid", were introduced in China. These standards set requirements for PV power plant quality, Low Voltage Ride Through (LVRT), safety and protection, metering, technical conditions, and anti-islanding (AI). Standard Q/GDW 617-2011 specified that medium and large type power stations have LVRT capability. In addition PV power system shall assure continuous operation with the grid whilst the connected point of voltage is within the voltage range provided in Fig. 2.4(a) Shown below. If that voltage is outside the range as specified in Table 2.7, the PV power system should discontinue supply of power to the grid.

The standard Q/GDW 618-2011 outlines the requirements when there is a grid voltage drop of $20 \%$ of nominal voltage, the PV power station shall assure connection to the grid for at least $1 \mathrm{~s}$; with the upper limit being a voltage drop of $90 \%$ nominal voltage and time of $3 \mathrm{~s}$.

It is suggested that PV power stations provide dynamic reactive support during LVRT, but not required. There are no requirements for power quality during a fault, however 
current distortion should be kept to a minimum. In the case when there is a fault or failure, PV power stations should follow timing and connection requirements. In addition active power should be restored as quickly as possible after the fault or failure desists. The active power should be restored to the value before the failure occurred, with a rate of power change of $10 \%$ rated power per second.

Table 2.8 provides a comparison of national standards and grid codes for select countries; in addition the graph of Fault Ride through requirements, for various countries have been outlined in Fig 2.4.

\section{Recommendations:}

It can be deduced from the above sections that the variety of standards and grid codes across the world, poses a unique barrier to the future growth of PV industry. Industry designers have to build, validate and sell various units for each national market. These practices drive up the cost for PV systems, and hamper the growth of the industry. The lack of transparent standards and codes, (which are generally not universally applied within applicable markets), add to the technical problems that arise with PV systems. The different languages that standards and grid codes are written in, cause reports to be inaccessible to certain parts of the industry. Also necessary to improve upon standards and grid codes, there needs to be cooperation between national governments, industry, distribution and transmission operators; which will remedy the administrative bottlenecks opposing PV industry expansion, as evidenced by a published document from the European Commission Renewable Energy Progress Report: "the problems of gaining connection to the electricity grid often result from a lack of adequate rules on grid connection and from a failure to dedicate sufficient administrative resources to process applications. Technical problems are also disruptive, with limited capacity of the grid to incorporate more variable renewable electricity and a general lack of strategy to address the problem. There are also financial constraints, with different and often opaque connection charging rules and 
Table 2.8: Examples of standards and grid codes for DER integration

\begin{tabular}{|c|c|c|c|c|}
\hline Standard & $\begin{array}{l}\text { Technology } \\
\text { Addressed }\end{array}$ & Voltage Ride-Through & $\begin{array}{l}\text { Ride-Through Contribu- } \\
\text { tion }\end{array}$ & $\begin{array}{l}\text { Frequency Ride- } \\
\text { Through }\end{array}$ \\
\hline 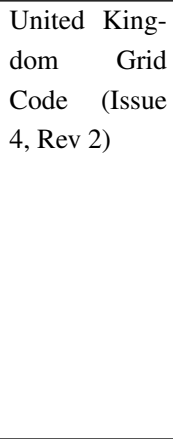 & $\begin{array}{l}\text { Solar, } \\
\text { geother- } \\
\text { mal, wave, } \\
\text { tidal, wind } \\
\text { or similar. } \\
\text { Solar, wind, } \\
\text { and wave are } \\
\text { mentioned to } \\
\text { as Intermit- } \\
\text { tent Power } \\
\text { Sources. }\end{array}$ & $\begin{array}{l}\text { Fault ride-through requirements may } \\
\text { not be the same for different types } \\
\text { of renewable generations. Unwanted } \\
\text { island process should be avoided by } \\
\text { tripping if the voltage at } \mathrm{PCC} \leqslant \\
0.8 \mathrm{pu} \text { for more than } 2 \mathrm{~s} \text {; or } \geqslant 1.2 \mathrm{pu} \\
\text { for more than } 1 \mathrm{~s} \text {. }\end{array}$ & $\begin{array}{l}\text { For an intermittent gener- } \\
\text { ation, active power ought } \\
\text { to return to within } 90 \% \text { of } \\
\text { the available active power } \\
\text { within } 1 \text { second of the volt- } \\
\text { age returning within the } \\
\text { normal range. }\end{array}$ & $\begin{array}{l}\text { fault ride-through } \\
\text { requirements may } \\
\text { not be the same for } \\
\text { different types of } \\
\text { renewable generations. } \\
\text { Unwanted island pro- } \\
\text { cess should be avoided } \\
\text { by tripping if the } \\
\text { frequency } \geqslant 52 \mathrm{~Hz} \text { or } \\
\leqslant 47 \mathrm{~Hz} \text { for more than } \\
2 \mathrm{~s} \text {. }\end{array}$ \\
\hline $\begin{array}{l}\text { Australian } \\
\text { National } \\
\text { Electric- } \\
\text { ity Market } \\
\text { (NEM) - } \\
\text { Minimum } \\
\text { Connection } \\
\text { Standards }\end{array}$ & $\begin{array}{l}\text { All technolo- } \\
\text { gies }\end{array}$ & $\begin{array}{l}\text { Voltage at the PCC: } \mathrm{V} \geqslant 0.90 \text { p.u. } \\
\text { and, } \mathrm{V} \leqslant 1.10 \text { p.u.- operating con- } \\
\text { tinuously as long as ratio of volt- } \\
\text { age to the frequency at } \mathrm{PCC}(\mathrm{V} / \mathrm{f}) \leqslant \\
1.15 \text { for two minutes and } \leqslant 1.1 \text { for } \\
\text { ten minutes. }\end{array}$ & $\begin{array}{l}\text { operating continuously for } \\
\text { credible contingency event, } \\
\text { a single phase to phase, and } \\
\text { two-phase fault except for } \\
<100 \mathrm{MW} \text { and no harm- } \\
\text { ful impact on power quality } \\
\text { and security of power sys- } \\
\text { tem. }\end{array}$ & $\begin{array}{l}\text { Different values for } \\
\text { different regions, ride- } \\
\text { through set times of } \\
9 \text { seconds, } 2 \text { minutes, } \\
\text { and } 10 \text { minutes, except } \\
\text { for rate of change of } \\
>1 \mathrm{~Hz} / \text { second }\end{array}$ \\
\hline $\begin{array}{l}\text { Independent } \\
\text { Electricity } \\
\text { System Op- } \\
\text { erator in } \\
\text { Canada }\end{array}$ & $\begin{array}{l}\text { Generator fa- } \\
\text { cilities > } 50 \\
\text { MW or gen- } \\
\text { erator units > } \\
10 \mathrm{MW} \text {. }\end{array}$ & $\begin{array}{l}\text { Voltages for continuous operation: } \\
115-\mathrm{kV}, 113-127 \mathrm{kV}, 230-\mathrm{kV}, 220- \\
250 \mathrm{kV}, 500-\mathrm{kV}, 490-550 \mathrm{kV} \text {. For } \\
30 \text { minutes, the upper value can go } \\
\text { beyond the limits in northern On- } \\
\text { tario. Voltage Limits for maximum } \\
\text { of } 30-\text { minute: } 115-\mathrm{kV} \text { : } 132, \mathrm{kV} ; 230- \\
\mathrm{kV} \text { : } 260 \mathrm{kV} \text {. Ride-through routine } \\
\text { switching and design contingencies } \\
\text { except disconnected by configura- } \\
\text { tion. Connection Assessment and } \\
\text { Approval Process provides specific } \\
\text { connections requirements. }\end{array}$ & & $\begin{array}{l}\text { The continuous opera- } \\
\text { tion when } 59.4 \mathrm{~Hz} \leqslant \\
\text { frequency } \leqslant 60.6, \mathrm{~Hz} \text {, } \\
\text { and for a limited period } \\
\text { of time in these condi- } \\
\text { tions }(0.0 \mathrm{~s}, 57.0 \mathrm{~Hz}) \text {, } \\
(3.3 \mathrm{~s}, 57.0 \mathrm{~Hz}) \text { and } \\
(300 \mathrm{~s}, 59.0 \mathrm{~Hz}) \text {. }\end{array}$ \\
\hline $\begin{array}{l}\text { Australian } \\
\text { National } \\
\text { Electric- } \\
\text { ity Market } \\
\text { (NEM) - } \\
\text { Automatic } \\
\text { Connection } \\
\text { Standards }\end{array}$ & $\begin{array}{l}\text { All technolo- } \\
\text { gies }\end{array}$ & $\begin{array}{l}\text { Voltage at the POI: } \mathrm{V} \geqslant 0.90 \text { p.u. } \\
\text { - Continuous } 0.80 \text {,p.u. } \leqslant \mathrm{V}<0.90 \\
\text { p.u. }-10 \text { s } 0.70 \text { p.u. } \leqslant \mathrm{V}<0.80 \text { p.u. } \\
\text { - } 2 \text { s } 0.80 \text { p.u. } \leqslant \mathrm{V}<0.90 \text { p.u. }-10 \\
\text { s } .00 \text { p.u. } \leqslant \mathrm{V}<1.10 \text { p.u. - Con- } \\
\text { tinuous } 1.10 \text { p.u. } \leqslant \mathrm{V}<1.30 \text { p.u. - } \\
\text { varies linearly between from } 0.06 \text { s to } \\
0.9 \text { s } 1.3 \text { p.u. } \leqslant \mathrm{V} \text { - Instantaneous }\end{array}$ & $\begin{array}{l}\text { Reactive balance control } \\
\text { for at least the greater of } \\
\text { its pre-disturbance reactive } \\
\text { current and } 4 \% \text { of the max- } \\
\text { imum continuous current of } \\
\text { the generator for each } 1 \% \\
\text { reduction (from its pre-fault } \\
\text { level) of connection point } \\
\text { voltage during the fault. } \\
\text { From } 100 \text { ms after discon- } \\
\text { nection of the faulted el- } \\
\text { ement, active power of at } \\
\text { least } 95 \% \text { of the level exist- } \\
\text { ing just prior to the fault }\end{array}$ & $\begin{array}{l}\text { Different values for } \\
\text { different Regions, } \\
\text { Ride-through set times } \\
\text { of } 2 \text { minutes and } 10 \\
\text { minutes, except for } \\
\text { rate of change of } \\
>4 \mathrm{~Hz} / \mathrm{s} \text { for,more than } \\
0.25 \mathrm{~s}\end{array}$ \\
\hline Mexico & & $\begin{array}{l}\text { For a } 150 \mathrm{~ms} \text { zero voltage fault the } \\
\text { generator should not trip. }\end{array}$ & Not Addressed & $\begin{array}{l}\text { The continuous oper- } \\
\text { ation when } 57.5 \mathrm{~Hz} \\
>\text { frequency }>62 \mathrm{~Hz} \text {. } \\
\text { Immediate tripping } \\
\text { might happen at fre- } \\
\text { quency } \geqslant 62 \mathrm{~Hz} \text { or } \leqslant \\
57.5 \mathrm{~Hz} \text {. }\end{array}$ \\
\hline
\end{tabular}




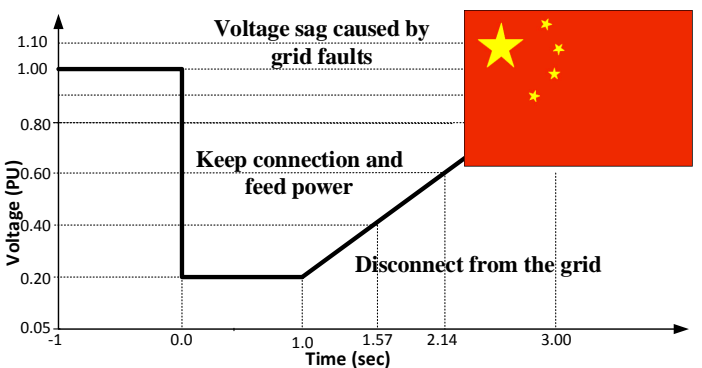

(a)

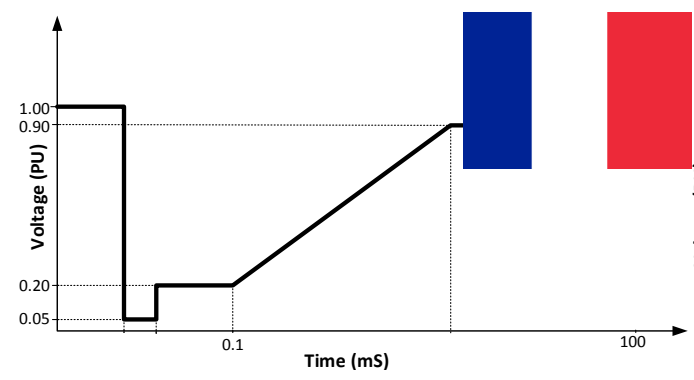

(c)

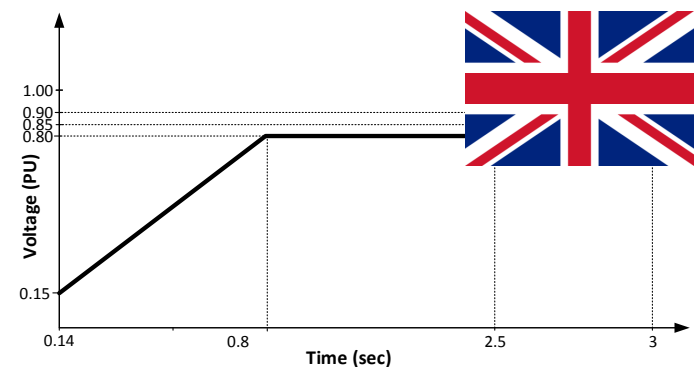

(e)

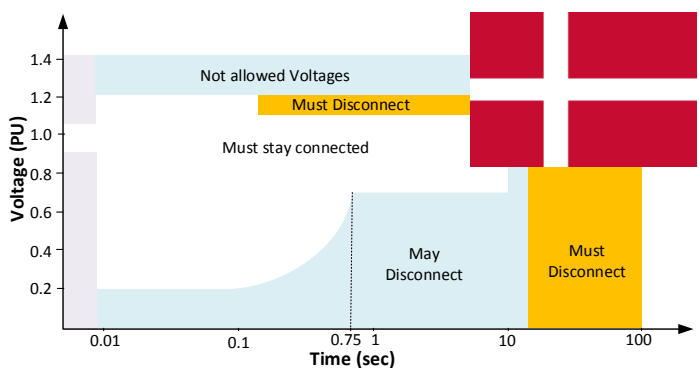

(b)

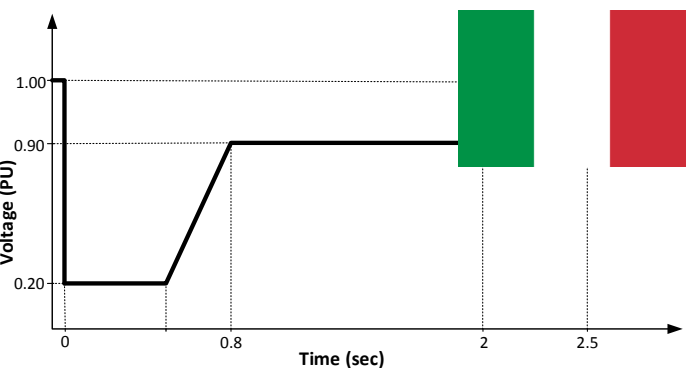

(d)

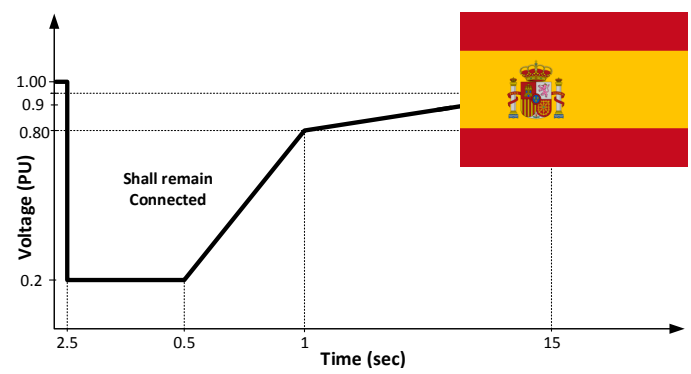

(f)

Figure 2.4: Fault Ride Through Requirements in Different Countries: (a) Denmark, (b) France, (c) Italy, (d) England, (e) Spain

risk of discrimination against smaller distributed power generators compared to large incumbent conventional energy producers."

Presented below are a compilation of select recommendations, from various industry players:

- Grid codes should be comprehensive and clear; have a set of commonly shared terms, and explicitly state requirements; thereby eliminating ambiguity 
- Requirements should be easily available from issuing body, and translated into the most common industry languages

- PV power requirements should not be discriminatory or disproportionate.

- Technical requirements should focus on vital parts of the systems performance, leaving openings for ancillary services.

- Requirements shall make a balance between benefits of the systems, cost, technical performance, and be identified so those requirements satisfy total minimum system cost.

- Drafting should only take place under pains of mandatory cooperation of all necessary parties. In addition after new standards or requirements are made official, there should be adequate transition time.

- Requirements should use as a point of reference the Point of Common Coupling which should be defined between Transmission System Operator (TSO)/ Distribution System Operator (DSO), and the generation/consumption unit.

\subsection{Topology Classification of Inverters}

This part of the chapter begins with an indication of some current power inverter topologies for combining PV modules to the electric power grid. The methods are further discussed and assessed to identify the most appropriate topologies for prospect PV inverters.

The former technology, shown in Fig. 2.5(a), was based on centralized inverters that attached a big amount of PV modules to the electric power grid.

The current technology contain the string inverters and the ac module [55]. The string inverter, shown in Fig. 2.5(b), is a condensed version of the centralized inverter, where a single string of PV modules is linked to the inverter. 


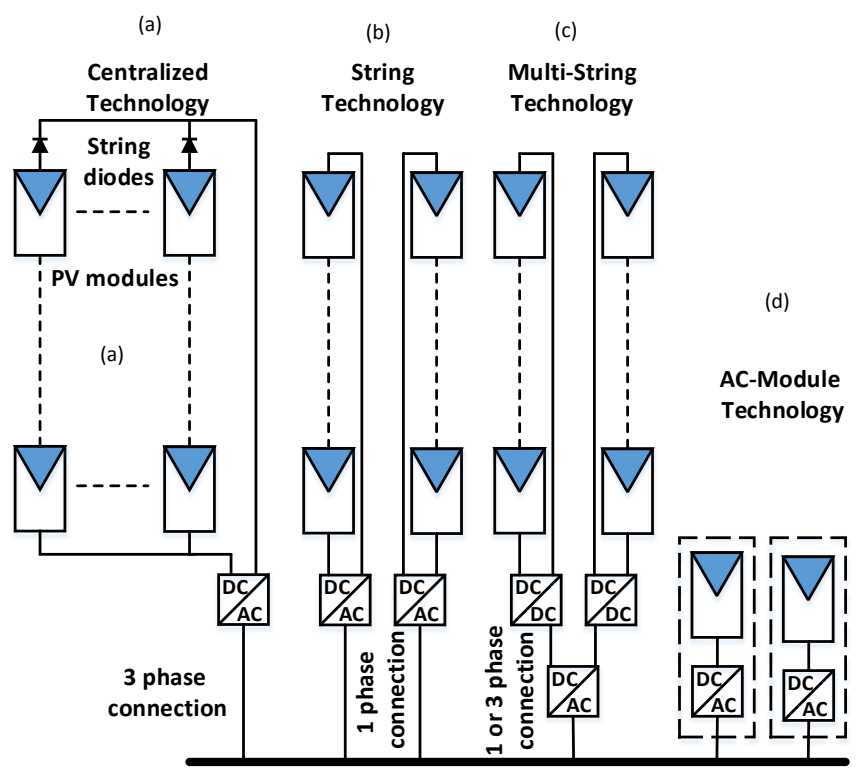

Figure 2.5: Summary of PV inverters technologies. (a) centralized (b) string (c) multistring (d) ac-module and ac cell technologies.

Fig. 2.5(d) represents the integration of the dc/AC power inverter and PV module into a single electrical apparatus. It eliminates the gap losses among PV modules since there is only one Photovoltaic module, in addition to supporting optimum adjustment between the inverter and PV module and, therefore, there is an individual maximum power point tracker (MPPT).

Fig. 2.5(c) shows a multi-string inverter which is the additional expansion of the string inverter. This type of inverter is used when numerous strings are interfaced with their own dc - dc converter and then those dc-dc converters are connected to a common dc - ac inverter [56].

A classification of diverse inverter designs is presented in this section. The topologies are classified based on the number of power processing steps, position of capacitors for decoupling the power, if they utilize transformers or not, and kinds of grid interface.

- Stages of Power Processing: 
a)

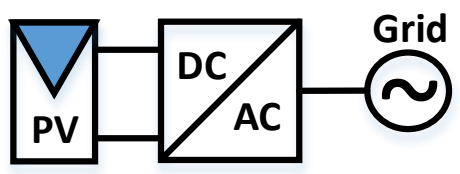

b)

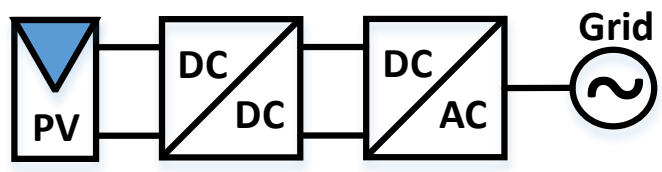

c)

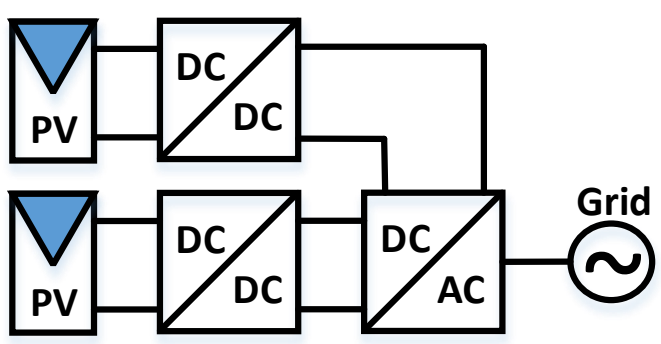

Figure 2.6: (a) Single power processing stage (For voltage amplification, grid current control, and MPPT) (b) Dual power processing inverter (dcac inverter controls the grid current, the dcdc converter is in charge of the MPPT, the Voltage amplification can be included in both stages. (c) Dual-stage inverter.

Fig. 2.6 displays three circumstances of multiple and single stage inverters. Fig. 2.6(a) represents a single-stage inverter. This inverter must take over all the responsibilities by itself, for example, grid current control, MPPT, and voltage increase. This configuration is the typical arrangement designed for a central inverter. [55] suggests that the inverter should design in a way that could be able to tolerate a peak power of double of the rated power.

A dual-stage inverter is illustrated in Fig. 2.6(b). The MPPT and possibly the voltage increase is are the $\mathrm{dc}-\mathrm{dc}$ converter responsibility. The dcdc converters output is either a pure dc voltage or modulated current to follow a rectified sine wave. When the converter is designed to control the rated power, the output is dc. When the dc-ac inverter is used for handling the grid current using bang-bang operation or pulse width modulation (PWM), the output is modulated current. In 
a)

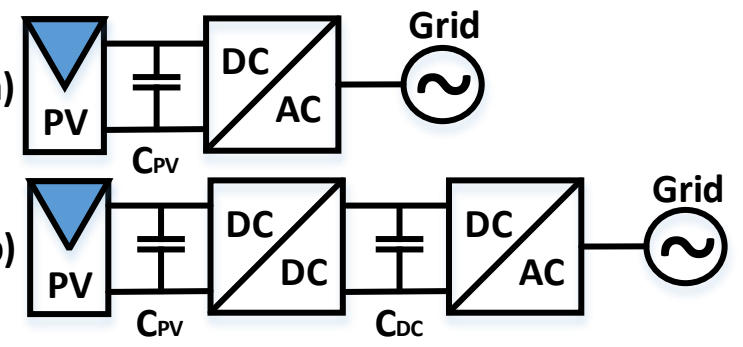

Figure 2.7: Power decoupling capacitor locations: (a) In parallel with the PV modules (in single-stage inverter applications). (b) in parallel with the dc-link or the PV modules (in multi-stage inverter applications)

the later case, the dcac inverter switching frequency is equal to the grid frequency, and the rectified current is transforming to a full sine-wave. Then the current control is managed by the dc dc converter. In this case, a high efficiency can be reached if the nominal power is low. Furthermore, PWM mode should be implemented for the grid-connected inverter if the rated power is high.

The multi-string inverter is illustrated in Fig. 2.6(c). The dc - dc converters are solely used for voltage increase and MPPT. The dc-link, which is responsible for controlling the grid current, is the point that dc-ac inverter and the dc-dc converter are connected. Using this method gives a better control of each PV string and the shared dc - ac inverter may be based on standard variable speed drive (VSD) technology.

- Power Decoupling:

is typically attained by using an electrolytic capacitor, which is the key factor in limiting the lifetime and should be remained as small as possible and can be substituted by film capacitors if possible. As shown in Fig. 2.7, the capacitor can be located either in the dc-link between the stages of inverter or in parallel with the PV modules. 


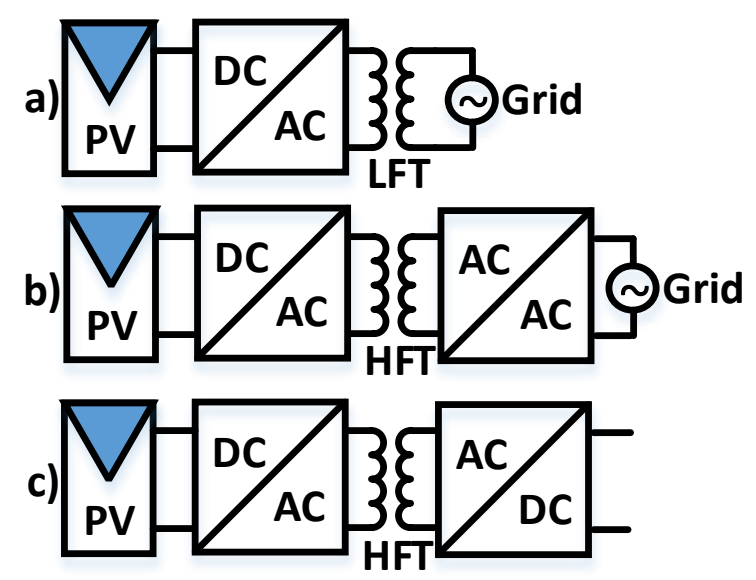

Figure 2.8: Examples of transformer-included inverter solutions. (a) Line-frequency transformer (LFT) is placed between the grid and the inverter (solves problems with injection of dc currents into the grid). (b) High-frequency transformer (HFT) is embedded in an HF-link grid-connected ac/ac inverter. (c) HFT is embedded in a dc-link PV-moduleconnected dcdc converter.

- Modes of Interconnections:

As illustrated in Fig. 2.8, some inverter applications use a line-frequency transformer on the way to the power grid, others use a high-frequency dc-ac inverter or dcdc converter with an implemented transformer, and finally, some topologies do not utilize transformers at all. Because of the large size of the e-frequency transformer, it is known as an inadequate component.

Just a small number of high-input-voltage transformerless topologies that can be grounded both at the input and at the output have been introduced in the literature [57, 58]. One configuration is illustrated in Fig. 2.9

\subsection{Three Phase Grid Connected Inverters}

In [59] a Multi-Functional Grid-Connected Inverter (MFGCI) has examined as shown in Fig. 2.10. three-level PWM achieved by employing an asymmetrical leg, similar to 


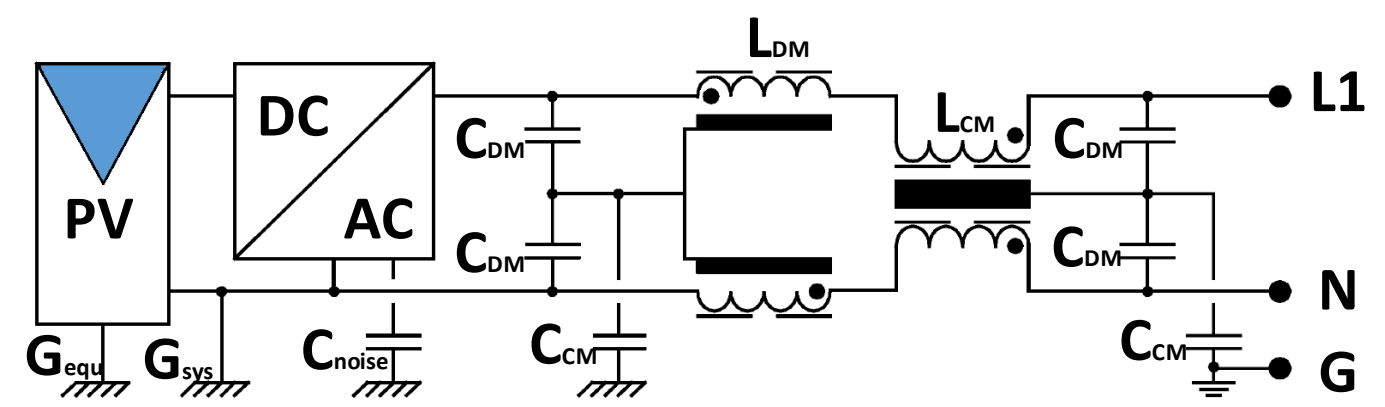

Figure 2.9: High-input-voltage transformerless PV inverter (with common-mode (CM) and differential mode (DM) EMI filters)

the single-phase H-bridge converter, that produces less voltage harmonic on the ac side compared with a two-level design. In three phase utility, this MFGI can be employed to as three autonomous voltage source inverters as shown in Fig. 2.10(a). It can also be used as a combined inverter as shown in Fig. 2.10(b), in which the dc-bus is shared with three cells and is fed by renewable energy sources and/or energy storage devices.

A three-phase MFGCI configuration utilizing soft-switching technology is shown in Fig. $2.11[60,61]$. This formation generally contains auxiliary active resonant commutated snubber link auxiliary (ARCSL), PV array, and LCL-filter. However, the filter can be newly proposed filters like $L L C L$ or $L(L C L)_{2}[8,9,62]$. An active power filter for integrated grid-connected PV applications and related controllers are proposed in [63]. Eliminating the harmonics and providing power from the PV are two purposes of the controller.

A cascaded multilevel grid-connected inverter for high voltage implementation and high power PV system is presented in [64-66]. low device rating, lesser electromagnetic interference, and improved power quality, modularity, etc. are the advantages of this topology for inverters. Considering a cascaded PV system, the output voltage from each individual converter module synthesizes the total ac output voltage in one phase leg that must satisfy grid codes or necessities. Unbalanced ac output voltage can be the result of 


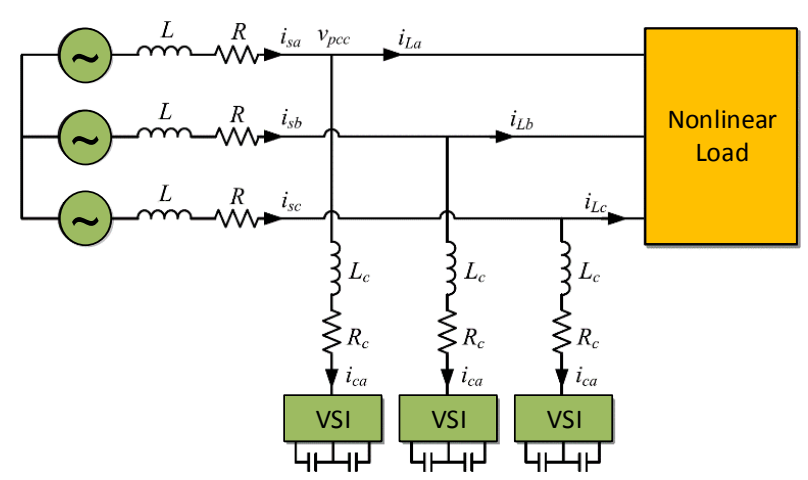

(a)

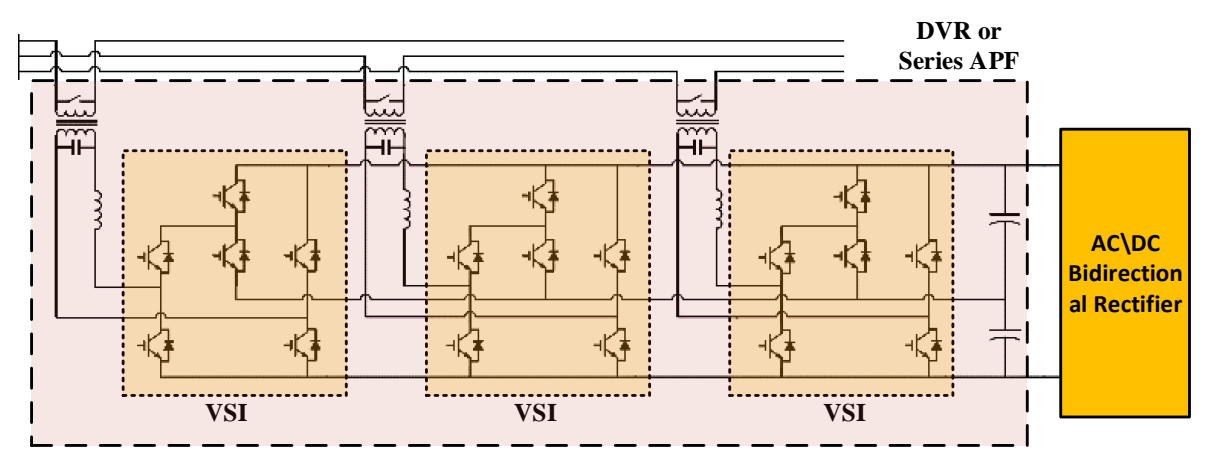

(b)

Figure 2.10: Topology and its of the MFGCI cell (a) application in three-phase utility (b) application in three-phase utility

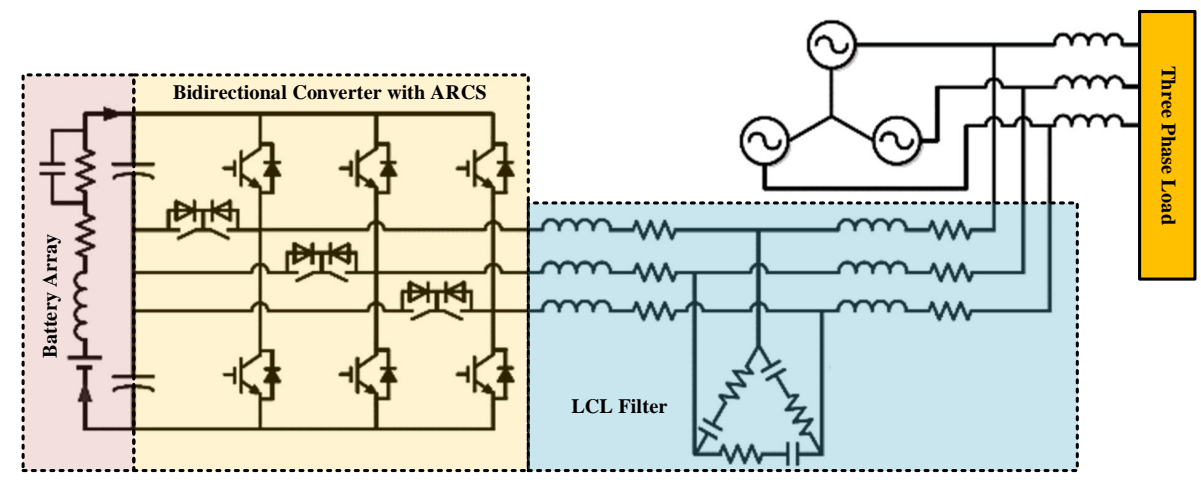

Figure 2.11: Utility connected bidirectional soft-switching MFGCI

active power incompatibility of these units. The reason is that the same grid current runs in the ac-side of each converter unit. 
Table 2.9: Comparisons of multi-functional three-phase grid-inverter topologies

\begin{tabular}{|c|c|c|c|c|c|c|c|c|}
\hline 苞 & Author & Topology & $\begin{array}{l}\text { Current } \\
\text { mode }\end{array}$ & $\begin{array}{l}\text { Modulation/ } \\
\text { control }\end{array}$ & Capacity & $\begin{array}{l}\text { Switching } \\
\text { fre- } \\
\text { quency } \\
(\mathrm{kHz})\end{array}$ & $\begin{array}{l}\text { Extra func- } \\
\text { tions }\end{array}$ & Application \\
\hline 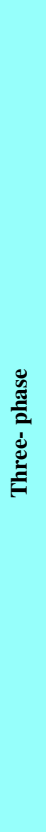 & $\begin{array}{l}\text { Mohod and Aware [67] } \\
\text { Marei et al. [68] } \\
\text { Cheng et al. [69] } \\
\text { Lv et al. [70] } \\
\text { Han et al. [71] } \\
\text { Li et al. [72] } \\
\text { Wang et al. [73] } \\
\text { Yu etal. [74] } \\
\text { Dasgupta et al. [75] } \\
\text { Cheng etal. [76] } \\
\text { Naderi etal. [77] } \\
\text { Sawant etal. [78] } \\
\text { Wang etal. [79] } \\
\text { Majumder etal. [80] } \\
\text { Gajanayake et al. [81] } \\
\text { Tsengenes and Adamidis } \\
\text { [82] } \\
\text { Saitou etal. [83] } \\
\text { Chandhaket etal. [61] } \\
\text { Abolhassani etal. [84] } \\
\text { Wuetal. [85] } \\
\text { He etal. [86] } \\
\text { Yu etal. [87] } \\
\text { Kim etal. [88] }\end{array}$ & $\begin{array}{l}\text { H-bridge } \\
\text { H-bridge } \\
\text { H-bridge } \\
\text { H-bridge } \\
\text { H-bridge } \\
\text { Four-bridge } \\
\text { Four-bridge } \\
\text { Four-bridge } \\
\text { H-bridge } \\
\text { H-bridge } \\
\text { H-bridge } \\
\text { Four-bridge } \\
\text { Four-bridge } \\
\text { Full-bridge } \\
\text { ZVI } \\
\text { Three-level NPC } \\
\text { H-bridge } \\
\text { H-bridge } \\
\text { H-bridge } \\
\text { H-bridge } \\
\text { Full-bridge } \\
\text { H-bridge } \\
\text { H-bridge }\end{array}$ & $\begin{array}{l}\text { Direct } \\
\text { Direct } \\
\text { Direct } \\
\text { Direct } \\
\text { Direct } \\
\text { Direct } \\
\text { Direct } \\
\text { Direct } \\
\text { Direct } \\
\text { Direct } \\
\text { Direct } \\
\text { Direct } \\
\text { Direct } \\
\text { Direct } \\
\text { Direct } \\
\text { Direct } \\
\\
\text { Direct } \\
\text { Direct } \\
\text { Direct } \\
\text { Direct } \\
\text { Direct } \\
\text { Direct } \\
\text { Direct }\end{array}$ & $\begin{array}{l}\text { Hysteresis } \\
\text { SPWM/FLC,PI } \\
\text { SPWM/droop } \\
\text { control } \\
\text { SPWM/PI } \\
\text { SPWM/PI } \\
\text { SPWM/PI } \\
\text { SPWM/PI,PR } \\
\text { SPWM/PI } \\
\text { SPWM/Lyapunov } \\
\text { - } \\
\text { Hysteresis } \\
\text { 3D-SVPWM } \\
\text { SPWM/PI } \\
\text { Hysteresis/LQR } \\
\text { SVPWM/PI } \\
\text { SVPWM/PI } \\
\text { SPWM/PI } \\
\text { SPWM/PI } \\
\text { SPWM/PI } \\
\text { SPWM/PI } \\
\text { SPWM/repetitive } \\
\text { SPWM/PI } \\
\text { Hysteresis }\end{array}$ & $\begin{array}{l}50 \mathrm{kVA} \\
- \\
1 \mathrm{kVA} \\
400 \mathrm{kVA} \\
30 \mathrm{kVA} \\
- \\
- \\
- \\
75 \mathrm{VA} \\
5 \mathrm{kVA} \\
- \\
- \\
1 \mathrm{kVA} \\
- \\
1 \mathrm{kVA} \\
- \\
\\
- \\
20 \mathrm{kVA} \\
7.5 \mathrm{kVA} \\
1.1 \mathrm{kVA} \\
5 \mathrm{kVA} \\
10 \mathrm{kVA} \\
-\end{array}$ & $\begin{array}{l}- \\
- \\
20 \\
12.8 \\
10 \\
10 \\
16 \\
- \\
- \\
- \\
- \\
10 \\
10 \\
- \\
- \\
- \\
15 \\
- \\
- \\
20 \\
- \\
- \\
20\end{array}$ & $\begin{array}{l}\text { APF } \\
\text { APF } \\
\text { UC }^{3} \\
\text { APF } \\
\text { APF,ISWC } \\
\text { ISWC } \\
\text { UPQC } \\
\text { ISWC,APF } \\
\text { APF } \\
\text { PFC, UPS } \\
\text { APF,RPI } \\
\text { APF,UC } \\
\text { APF } \\
\text { APF,UC } \\
\text { APF } \\
\text { APF } \\
\text { RPI } \\
\text { PWM } \\
\text { APF } \\
\text { APF } \\
\text { APF } \\
\text { APF,RPI } \\
\text { APF }\end{array}$ & $\begin{array}{l}\text { Battery } \\
\text { Micro-source } \\
\text { Micro-source } \\
\text { Micro-source } \\
\text { WT } \\
\text { Micro-source } \\
\text { Micro-source } \\
\text { Micro-source } \\
\text { Micro-source } \\
\text { PV } \\
\text { Micro-source } \\
\text { PMSG } \\
\text { PV } \\
\text { Micro-source } \\
\text { Micro-source } \\
\text { PV } \\
\text { Battery } \\
\text { Battery } \\
\text { DFIG } \\
\text { PV } \\
\text { Micro-source } \\
\text { Micro-source } \\
\text { PV }\end{array}$ \\
\hline
\end{tabular}

The reactive power, harmonic and unbalance current can also be compensated by the MFGCI. In order to ease the procedure of reference current generation, multi-functional grid-connected inverters (MFGCIs) mostly use direct current control. Table 2.9 and Table 2.10 display a thorough assessment of different kinds of grid connected inverter's topologies in three-phase and single-phase applications, respectively.

${ }^{3}$ Dynamic voltage regulator

${ }^{4}$ Unified power quality conditioner

${ }^{5}$ Power factor correction

${ }^{6}$ Uninterrupted power source

${ }^{7}$ Real power injection

${ }^{8}$ Harmonic voltage compensation

${ }^{9}$ Active power filter

${ }^{10}$ Unbalance compensation

${ }^{11}$ Voltage interruption/sag/swell compensation 
Table 2.10: Comparisons of multi-functional single-phase grid-inverter topologies.

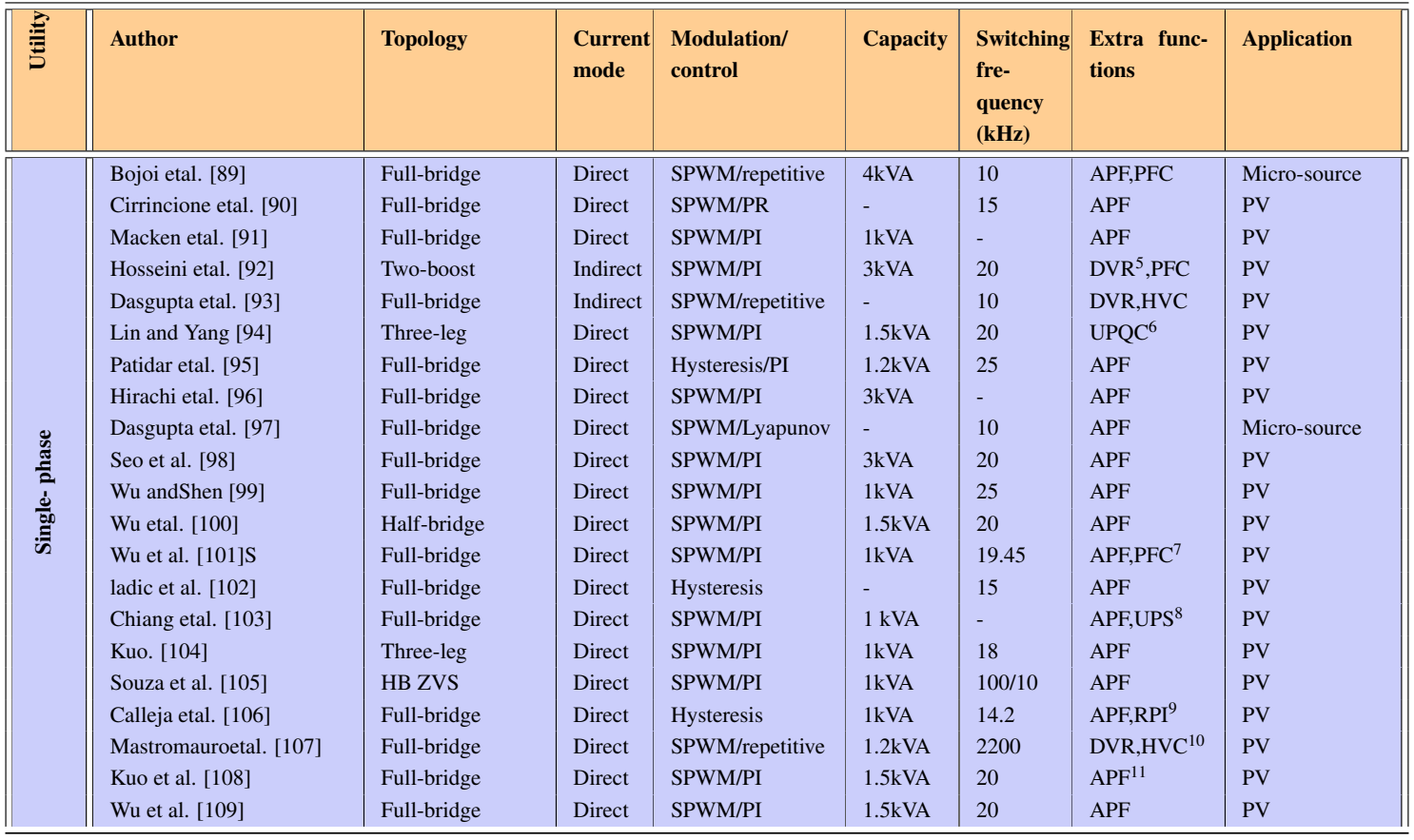

\subsection{Control of Grid Connected PV Systems}

In order to connect the PV system to the grid, controlling the power conditioning devices are the most important task that should be implemented. The controlling systems have two major parts.

\subsubsection{Renewable side controllers}

These controllers are mostly implemented for maximum power point tracking systems to extract the most from distributed sources. Moreover, these controllers may be used to protect the converters. 


\subsubsection{MPPT Control}

MPPT plays a key role in the performance of a PV system [110,111]. Many MPPT methods have been implemented and developed based on different approaches, like computational models used in machine learning [11], fuzzy and vary in complexity, popularity, required measurements, convergence speed, implementation hardware, cost and range of effectiveness.

A comprehensive review was conducted in [112] for many different MPPT methods introduced in the literature.

In Table 2.11 the main features of all the MPPT methods are presented.

\subsubsection{Grid-side controllers}

that are being used to perform active power control, reactive power control, DC-link voltage control, power quality control, and grid synchronization. These are the basic features for grid-connected inverters. However, some utilities may request the additional service like compensation of harmonics, voltage regulation, local frequency, etc.

\subsubsection{Active and Reactive Power Control}

Active power generation at low demand situations can cause overloading of the transformer or serious levels of grid voltage increase. On the other hand, the most important preventive cause to avoid the PV integration into the grid distribution networks is voltage rise [113]. Nominal or maximum power is provided by PV inverters during a limited time and the remaining power capacity of the inverter can be utilized for the voltage control

purposes. Active power curtailment and reactive power control are two economical and technically feasible solutions [114]. 
Table 2.11: Examples of Different MPPT Methods

\begin{tabular}{|c|c|c|c|c|c|c|c|}
\hline MPPT Technique & $\begin{array}{l}\text { PV Ar- } \\
\text { ray De- } \\
\text { pendent }\end{array}$ & $\begin{array}{l}\text { True } \\
\text { MPPT }\end{array}$ & $\begin{array}{l}\text { Analog } \\
\text { or } \\
\text { Digital }\end{array}$ & $\begin{array}{l}\text { Periodic } \\
\text { Tuning }\end{array}$ & $\begin{array}{l}\text { Converg } \\
\text { Speed }\end{array}$ & cComplexity & $\begin{array}{l}\text { Sensed } \\
\text { Param- } \\
\text { eters }\end{array}$ \\
\hline Hill-climbing/P\&O & No & Yes & Both & No & Varies & Low & $\begin{array}{l}\text { Voltage, } \\
\text { Current }\end{array}$ \\
\hline IncCond & No & Yes & Digital & No & Varies & Medium & $\begin{array}{l}\text { Voltage, } \\
\text { Current }\end{array}$ \\
\hline Fractional $V_{o c}$ & Yes & No & Both & Yes & Medium & Low & Voltage \\
\hline Fractional $I_{s c}$ & Yes & No & Both & Yes & Medium & Medium & Current \\
\hline Fuzzy Logic Control & Yes & Yes & Digital & Yes & Fast & High & Varies \\
\hline Neural Network & Yes & Yes & Digital & Yes & Fast & High & Varies \\
\hline RCC & No & Yes & Analog & No & Fast & Low & $\begin{array}{l}\text { Voltage, } \\
\text { Current }\end{array}$ \\
\hline Current Sweep & Yes & Yes & Digital & Yes & Slow & High & $\begin{array}{l}\text { Voltage, } \\
\text { Current }\end{array}$ \\
\hline $\begin{array}{l}\text { DC Link Capacitor Droop Con- } \\
\text { trol }\end{array}$ & No & No & Both & No & Medium & Low & Voltage \\
\hline Load I or V Maximization & No & No & Analog & No & Fast & Low & $\begin{array}{l}\text { Voltage, } \\
\text { Current }\end{array}$ \\
\hline dP/dV or dP/dI Feedback Control & No & Yes & Digital & No & Fast & Medium & $\begin{array}{l}\text { Voltage, } \\
\text { Current }\end{array}$ \\
\hline Array Reconfiguration & Yes & No & Digital & Yes & Slow & High & $\begin{array}{l}\text { Voltage, } \\
\text { Current }\end{array}$ \\
\hline Linear Current Control & Yes & No & Digital & Yes & Fast & Medium & Irradiance \\
\hline$I_{m p p}$ and $V m p p$ Computation & Yes & Yes & Digital & Yes & N/A & Medium & $\begin{array}{l}\text { Irradiance } \\
\text { Temper- } \\
\text { ature }\end{array}$ \\
\hline State-based MPPT & Yes & Yes & Both & Yes & Fast & High & $\begin{array}{l}\text { Voltage, } \\
\text { Current }\end{array}$ \\
\hline OCC MPPT & Yes & No & Both & Yes & Fast & Medium & Current \\
\hline BFV & Yes & No & Both & Yes & N/A & Low & None \\
\hline LRCM & Yes & No & Digital & No & N/A & High & $\begin{array}{l}\text { Voltage, } \\
\text { Current }\end{array}$ \\
\hline Slide Control & No & Yes & Digital & No & Fast & Medium & $\begin{array}{l}\text { Voltage, } \\
\text { Current }\end{array}$ \\
\hline Voltage Oriented Control & Yes & Yes & Digital & No & Medium & High & $\begin{array}{l}\text { Voltage, } \\
\text { Current }\end{array}$ \\
\hline Extremum Seeking Control & Yes & Yes & Digital & Yes & Fast & Medium & $\begin{array}{l}\text { Voltage, } \\
\text { Current }\end{array}$ \\
\hline
\end{tabular}


The amount of reactive power that can be provided by a PV system is limited. The voltage might not maintain in the satisfactory limits when active power production is high. This may happen because of inadequate inverters capacity for a reactive portion. Furthermore, the reactive power effectiveness in low voltage (LV) networks is limited due to bigger $\mathrm{R} / \mathrm{X}$ values. In order to prevent the high voltage limit violation, it is essential to curtail the active power. Different techniques for power curtailment are suggested by $[113,115,116]$. These techniques are costly for the PV panel owner because of cutting generated power. Therefore, optimal usage of present reactive power can lead to decrease the number of power curtailments.

Local Control: Among many different proposed methods for reactive power control, three techniques are mostly implemented: 1-local power generation measurements [113, 117, 118], 2- local voltage measurements method [113,117,119], and 3-combination of both. The reference voltage is defined by local measurement method for controller design of reactive voltage, as presented in [120]. If local parameters are not synced with the grid, the local control techniques may cause an extreme consumption of reactive power or maybe insufficient voltage support.

Centralized Control: The local control parameters were optimized by using a centralized optimization in [121]. Even though the PV output is in direct relationship with the cloud covering situations, optimum points of operations are achieved by tuning the local controllers.

\section{Cascaded Multilevel Converters Control}

A cascaded multi-level PV system for large-scale PV implementation is proposed in [122]. This system employed cascaded multilevel inverters and a current-fed dual-activebridge (CF-DAB) dc - dc converters and as shown in Fig 2.12. Separate control system for active and reactive power is designed for improving the system performance when it is operating. Each PV converter controls the reactive power in real-time to decrease the 


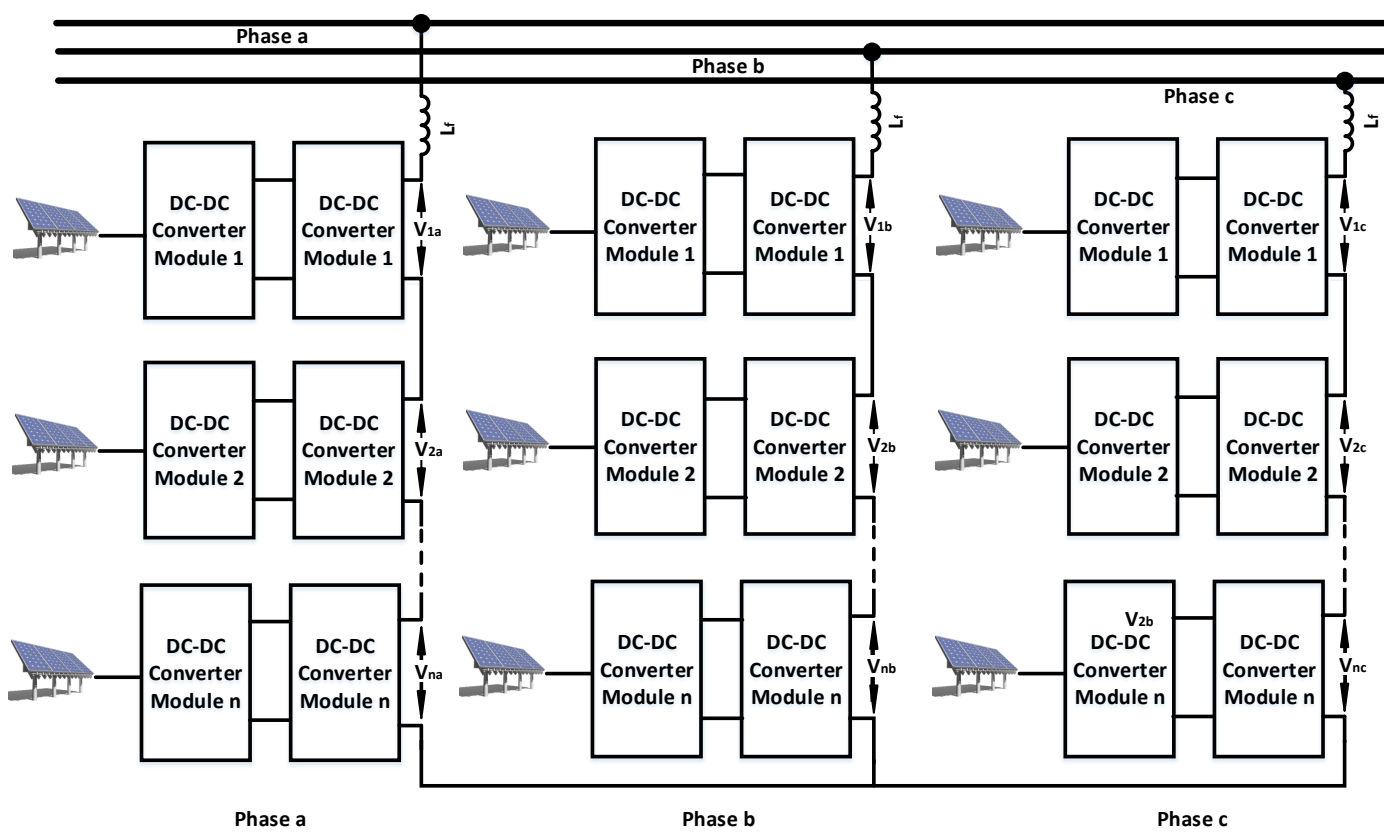

Figure 2.12: Grid connected PV system with cascaded multilevel converter

chance of the over-modulation, which may result in the asymmetrical active power output from the PV arrays. The system lifetime can be enhanced by placing film capacitors instead of electrolytic capacitors. A large low-frequency dc voltage ripple is allowed by the proposed PV system. This will not affect the MPPT attained by means of CF-DAB dcdc converters.

\subsubsection{Control Strategies}

Joining the photovoltaic system may cause some major problems like the instability of the grid and disturbances. By implementing control systems these effects can be mitigated properly [123]. Generally, controllers have six different categories based on their applications, which is summarized in Fig. 2.13.

1. Linear controllers have three sub categories 


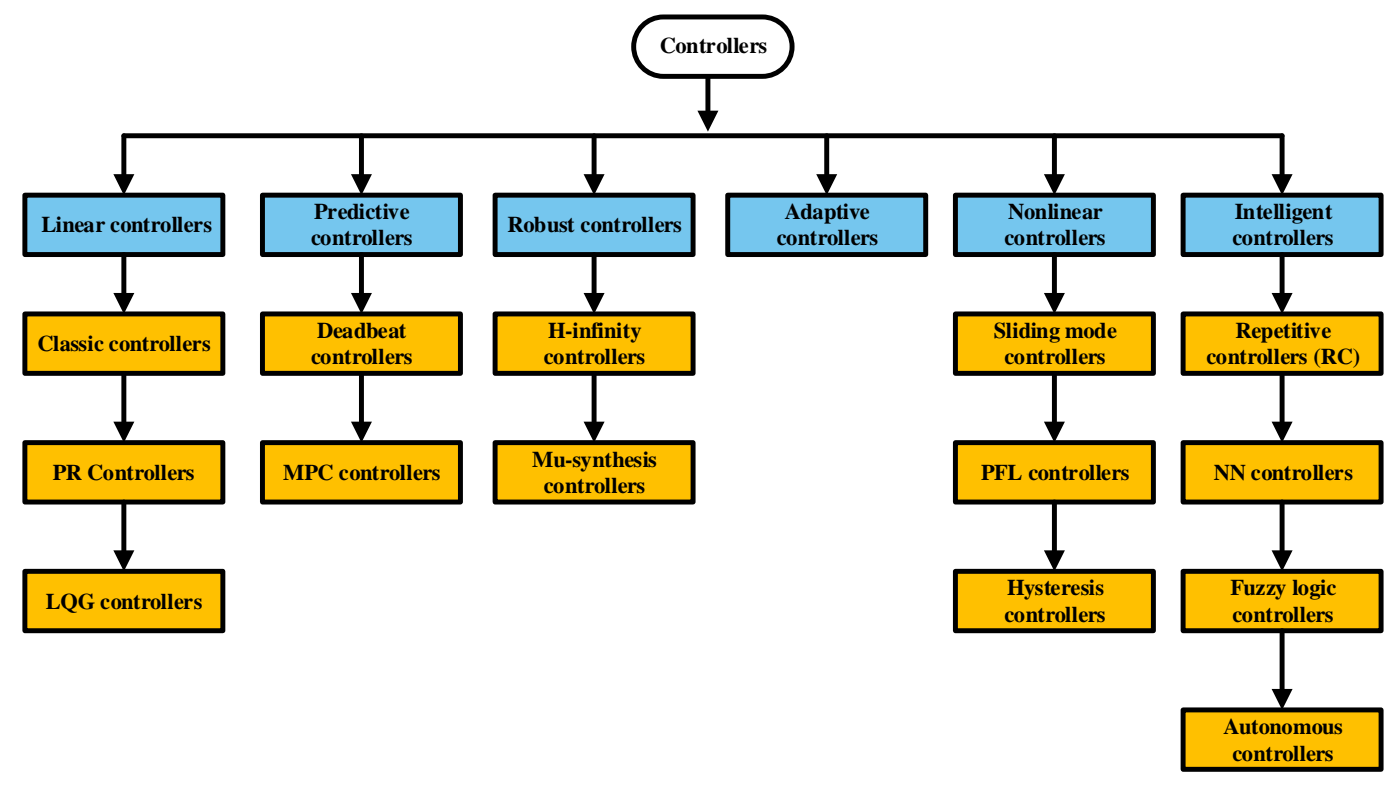

Figure 2.13: Summary of control strategies for Photovoltaic applications

- Classic controllers including: proportional, proportional-integral (PI), proportionalderivative (PD), and proportional-integral-derivative (PID) controllers).

- Proportional - Resonant (PR) controller [124]: the difference between classic PI controller and PR controller is how integration part perform. The PR controller only integrates frequencies close to the resonant frequency. Consequently, phase shift or stationary error is not included.

- Linear-quadratic Gaussian (LQG) controller is a mixture of the linear-quadratic regulator (LQR) and a Kalman filter. LQG control is applicable in both linear time-invariant systems and linear time-varying systems[4].

2. Nonlinear controllers: has more complicated design and implementation compared with linear controllers

3. Robust controllers: are designed by considering uncertainties to reach stability (when there are modeling errors) and robust performance, even in multivariable systems. 
- Sliding mode controllers (SMC) has been used for output voltage regulation. This kind of controller does not have a sensitivity to parameter variations and load disturbances. The limited sampling rate, chattering phenomenon, and degrading the total system efficiency are the drawbacks of this control method [4].

- Partial feedback linearization (PFL) controllers transform a nonlinear system to a linear system (partially or fully), therefore, design methods for a linear system can be implemented in this type of controller.

- Hysteresis controllers: in this controller a fixed switching frequency must be achieved by an adaptive band of the controller.

4. Adaptive controllers [4]: automatically regulate the controller based on the operational conditions of the system. Due to inaccurate parameters of the highperformance system, the complexity of the computation of the system is high.

5. Robust controllers: are designed by considering uncertainties to reach stability (when there are modeling errors) and robust performance, even in multivariable systems.

- H-infinity controllers: are used in multivariable systems. However, these controllers are highly complex in terms of computational analysis and they need a precise model of the system that is going to be controlled.

- Mu-synthesis controllers: can be implemented in order to take into account the influence of unstructured and structured uncertainties on the execution of the system.

6. Predictive controllers: forecast the upcoming performance of the controlled parameters. Predefined optimization criterion is used to reach the ideal performance. The 


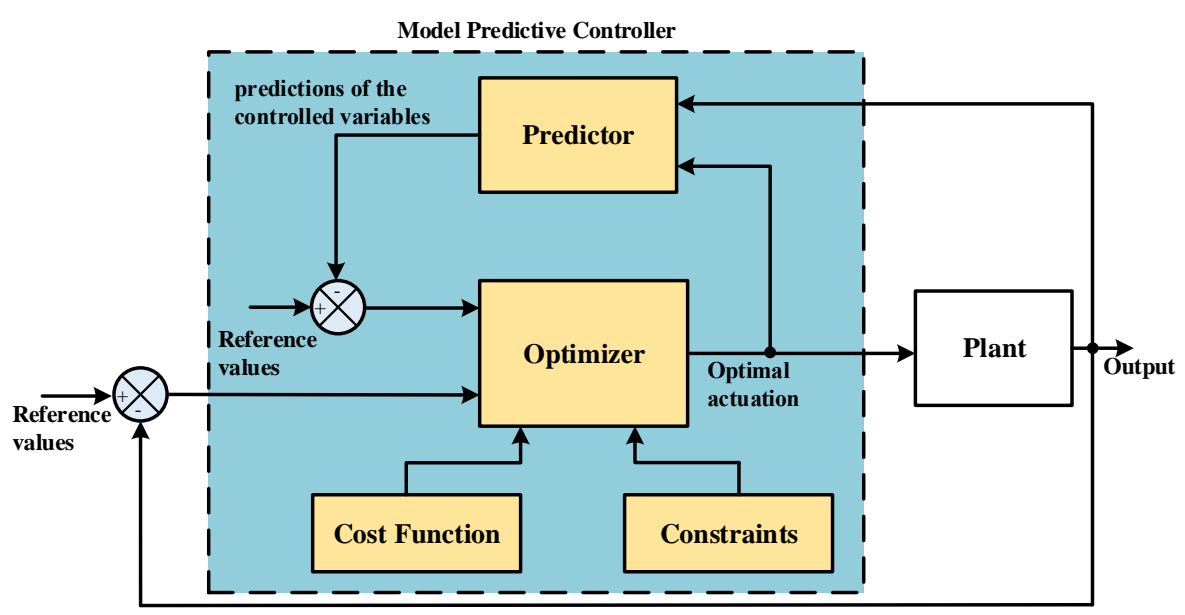

Figure 2.14: The general layout of a Model Predictive Control [4]

execution of this type of controller is easy, however, it has a rapid dynamic response and multivariable cases can be implemented.

- Deadbeat controllers: In this type of controller, the dynamic behavior of the system is defined by the differential equations. Then, the control signals are estimated for the state variables to achieve the references at for each sampling period.

- Model predictive control (MPC): uses a cost function criteria which needs to be minimized in order to choose the ideal actions. The structure of this controller is illustrated in Fig 2.14. System constraints and nonlinearities can be easily included in the design of the controller $[4,125]$.

7. Intelligent controller: is a class of controller that implements a kind of computing artificial intelligence methods which mostly were emulated the biological intelligence. A number of these methods are as follows. 


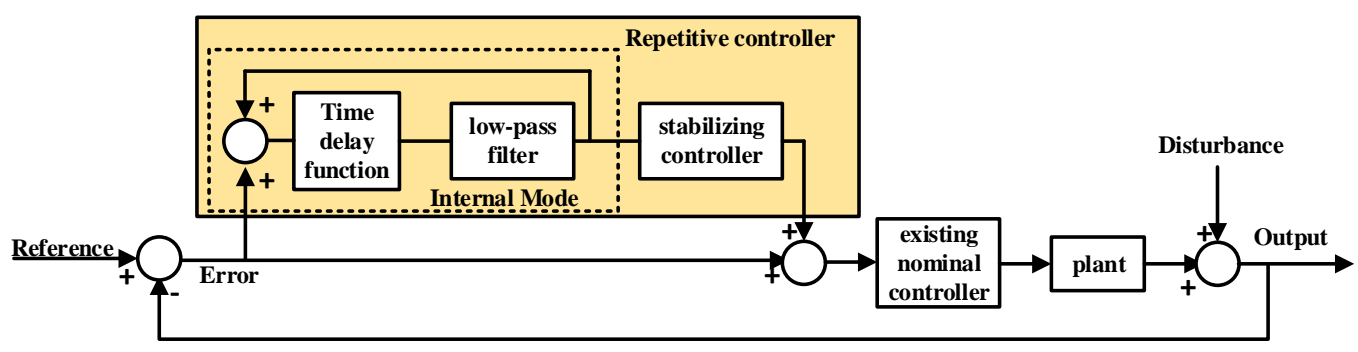

Figure 2.15: The general layout of a Repetitive Controller [5]

- Repetitive controllers (RC): use the internal model basics to track/reject any reference/disturbance which is injected in the closed-loop. The general structure of this controller is shown in Fig 2.15 [126].

- Neural network (NN) Controller: is a predictive and estimator controller that use a couple of artificial neurons that mimic brain system. This type of controller can be trained online or offline to provide the suitable inputs of the plant with the intention of obtaining desired outputs.

- Fuzzy logic controllers (FLC): is a decision making mathematical logic which has inputs with continuous values among 0 and 1 . This is in contrast to digital logic with discrete values of true or false (1 or 0$)$. FLC has the following components: 1- rule base which is a group of rules that defines how to control the system. 2- Fuzzification which is the procedure of translating the algebraic inputs to a kind that can be implemented by the inference mechanism. 3Information of the fuzzification are used by the inference mechanism. This mechanism chooses which rules are employed on the current status. 4- the decisions that are attained by the inference mechanism are transformed to a numerical input for the plant by defuzzification.

- Autonomous controllers: are able to accomplish complex tasks individually. High levels of computerization can be achieved by the addition of human 
knowledge and intelligence.

\subsubsection{Reference Frames}

In order to control inverters that are connected to the three-phase grid, designers are mostly using $d q$ reference frame or $\alpha \beta$ reference frame.

- $d q$ reference frame: Zero-direct-quadrature or simply $d q 0$ transform uses Park transformation to convert the $a b c$ frame to the $d q$ frame. By using this method the grid voltage and current waveforms will be converted to a rotational reference frame that has the same frequency of the grid. In this way, variables of the control system will be transformed into DC variables, which makes the controller design and filtering much easier. The general configuration of $d q$ reference frame controller is shown in Fig. 2.16.

- $\alpha \beta$ reference frame: Alpha-beta transform uses Clarke transformation to transform the $a b c$ frame or the single-phase frame to $\alpha \beta$-frame. A stationary reference frame is made by this transformation by using grid current. By using this method, the variables of the control system will be changed to sinusoidal variables. The general configuration of the Alpha-beta reference frame is illustrated in Fig 2.17.

\subsection{Energy Storage in Photovoltaic Systems}

Higher solar integration requires implementation of battery (and super-capacitor) energy storage systems to compensate high energy (and high power) fluctuations caused by stochastic nature of renewable resources [127]. Different methods for calculating the battery energy capacity to accommodate a specific PV penetration level with minimum cost has been studied in the literature $[128,129]$. Increased use of renewable energies, es-

pecially PV, has resulted in bigger implementation of battery system in LV grid $[130,131]$. 


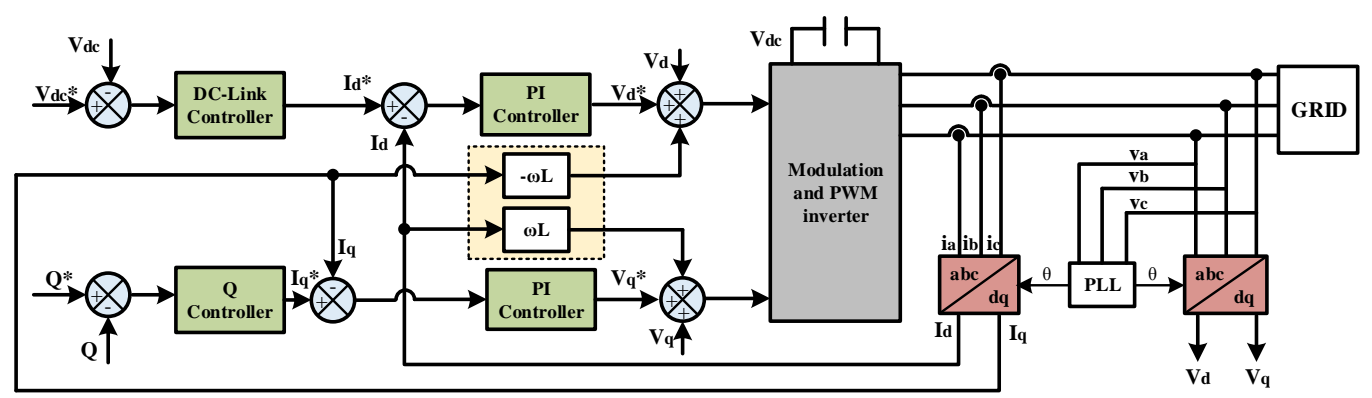

Figure 2.16: Typical configuration of synchronous rotating $(d q)$ reference frame control method [6].

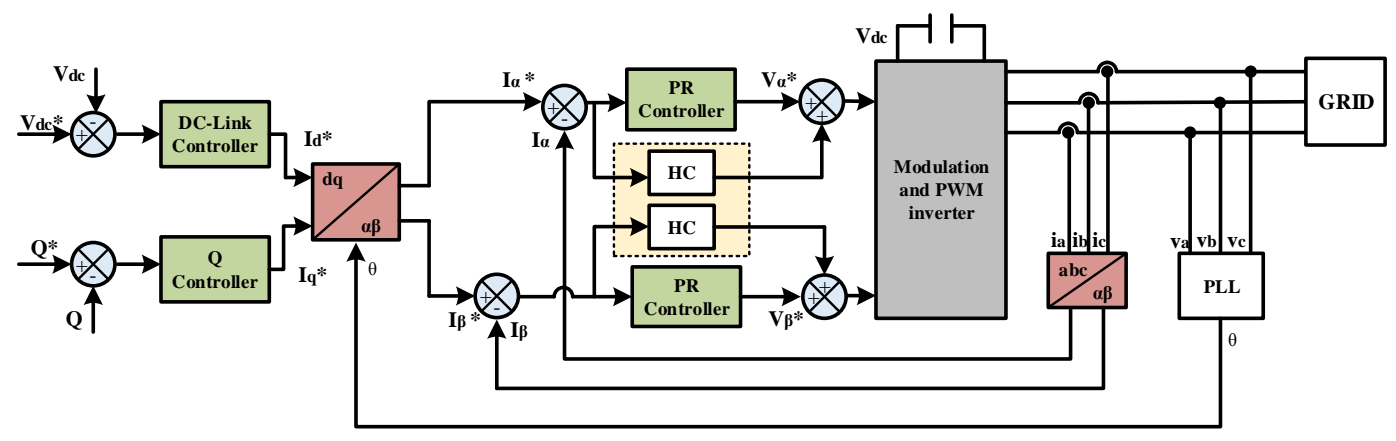

Figure 2.17: Typical configuration of synchronous rotating $(d q)$ reference frame control method [6].

Large scale integration of PV energy sources has a number of complications that need to be overcome as PV begins to compete and in time replace more traditional means of energy generation, i.e. coal, natural gas, and oil power plants.

In this section, we will be giving a brief description of energy storage systems as a whole, problems that are presented with the large scale integration of PV renewable energy, and the uses of energy storage systems to provide a means to resolve those problems.

\section{Energy Storage Systems:}

There are various types of energy storage systems (ESS) that can be used in conjunction with PV each of which has their uses in the electric grid. Examples of these ESS 
are: pumped hydro energy storage (PHES) [132-134], compressed air energy storage (CAES) [135-138, 138], flywheel energy storage (FES) [139-141], battery or electrochemical energy storage (EES) [142-146], flow battery energy storage (FBES) [147], superconducting magnetic energy storage (SMES) [148-155], and supercapacitors or dual layer capacitors (DLC) [156-161].

ESS can be used for multiple purposes depending on the discharge duration.

\section{Complications of PV Integration and Uses of Energy Storage:}

As PV energy sources becomes a higher percentage share of the total energy generation, complications due to PV integration become detrimental to the stability of the grid. This is where ESS come in to play. Using ESS effectively throughout the grid, in both the distribution and generation side of the grid will help provide stability and reliability to the grid.Energy storage applications in grid scale renewable energy systems has been studied in many studies $[131,162]$.

Voltage Support Voltage support is the ability to inject or absorb reactive power into/out of the transmission and distribution grid in order to maintain voltage levels at normal conditions. During high voltage levels, reactive power in volt ampere reactive (VAR) is removed from the grid. When the voltage sags, VARs are pumped in. Voltage support is normally handled by power facilities, in the case of PV power electronics need to be used in order to provide the necessary reactive power. However, ESS together with power electronics are best suited for voltage support for momentary imbalance in localized areas and is especially critical during peak demand when instability is worse in remote areas of the grid.

According to EPRI, most distribution system problems are due to voltage sags of $10 \%$ to $30 \%$ below nominal voltage, $120 \mathrm{~V}$ in the USA, with a duration of 3 to 30 cycles. With electronic equipment becoming more sensitive to voltage changes, 
only able to handle from a change of $10 \%$ to $5 \%$ or less from the rated voltage before becoming affected or damaged, voltage support is becoming much more critical.

Load Following Due to the inherent unpredictability in PV, such as that due to weather conditions, PV suffers from rapid loss in energy. When this happens, fast acting ESS such as SMES, FWES, and EES can take up production of energy for short periods of time, until PV energy production or an alternate means of generations can take up the load. Conversely, ESS in this capacity are also used to compensate for changes in load [163]. An increase in load will cause these ESS to discharge and provide energy and stability to the grid, while a decrease in load will cause ESS to charge and thus act as a load. The use of ESS in this capacity is called load following. Load following operates primarily on an hour to hour bases. It is especially useful in the morning and evening, when there are greater changes in the load. Load following is currently used mainly with more traditional means of generation as it takes time to ramp up production for a comparatively rapid increase in load.

Frequency Regulation Similar to load following, frequency regulation allows energy production to increase or decrease as needed in order to maintain a constant frequency in the case of rapid changing load or rapid changes in generation [164]. As $\mathrm{PV}$ is prone to such rapid changes in generation, frequency regulation is needed in maintaining the stability of the power grid. However, while load following deals primarily on the hour to hour level, frequency regulation works on a second to second level. When energy production needs to be decreased, ESS are set to charge and thus lowers the increased frequency due to over generation. When energy is needed, ESS discharge the stored energy and thus increasing the lowered frequency 
due to too larger demand. This stabilizes the frequency fluctuations due to the moment by moment viability of PV systems and load.

Time Shifting One of the main problems with PV is that peak power production does not occur during peak load times [132]. PV energy production is higher around 12:00 while peak load time is around 19:00 - 22:00. In order to meet this problem, ESS can be used for time shifting, also called load shifting. Time shifting is used when peak energy production does not match the time of the peak load. This is done by storing energy during the time when producing energy is less costly or during peak generation and using it to provide energy during peak load hours [165]. Without ESSs, the discrepancy between peak energy production and peak load times will cause utility companies to not be able to meet demand and require generators make up the energy needed which can be costly to utilities. ESS used for time shifting need to have discharge times ranging in the hours. ESS systems commonly used for these purposes are: PHS, CAES, EES, and FBES [166].

Seasonal/Long Term Storage Another problem with PV is the change in the amount of irradiance due to seasonal changes. Less energy can be produced during winter months as compared to summer months. This means that utilities need to find alternate means of providing energy to meet demand, usually the more traditional forms of energy generation. ESS can potentially solve this problem by providing seasonal storage which is the ability for energy storage systems to store energy for a prolonged period of time compensate long term disruptions in the supply, specifically for seasonal variability. For ESS to be able to provide seasonal storage, it needs to be able to store energy and provide energy for days, weeks, or months. At present, there is no ESS that can feasibly compensate for all of the energy loss in PV during seasons with lower irradiance due to the size and the cost of the needed system other than PHES and CAES. However, PV can be used to charge ESS to pro- 
vide time shifting, voltage support, load following, and frequency regulation. This lowers the demand on traditional generators and provides an opportunity for other forms of renewable energy, such as wind, to provide a steady amount of energy.

\subsection{Future Applications of PV Renewable Energy}

Distributed generation will lead to diverse sources which will average out the variation in the production of energy across the grid. Due to the lack of or the over development of renewable energy throughout the day, this source of power can be used in order to power battery electric vehicles. These battery electrical vehicles will require reaching longer distances and thus requiring more charging locations along the road. These renewable charging stations can be used to charge automotive batteries at battery charging stations [51].

The application of renewable energy to power and monitor electrical vehicles will not come at a cheap price [167]. Proliferation of electric vehicles at a large scale would require extensive infrastructure changes for production and optimization of vehicle components and maintenance of those components [168].

Increasing the power conversion efficiency is one of the cutting edge researches worldwide. This goal can by reducing the amount of material that is needed for each cell. Another approach can be implementing wider spectrum to produce electricity out of the sun. Silicon PV panels have efficiency rates of around 20\%. Researchers succeeded in producing power in both visible and near infrared regions of the solar array spectrum [169]. The utilization of the infrared segment of the spectrum of the sun can improve the efficiencies of solar by $30 \%$ or more. Harvesting the solar energy need space to install ground mounted PV panels in a large scale. A French company Ciel \& Terre International, developed floating solar in large scale on big bodies of water. Beside producing 
energy and saving land space, this method can reserve water by reducing the vaporization of water. Energy harvesting trees are another technology that will be used to save more space on the ground. Researchers are thinking of generating solar power in the space, where the sunshine is always available and the definition of the weather is completely different. Japanese Space Agency (JAXA) and Mitsubishi were able to transmit $10 \mathrm{~kW}$ power from a distance of 500 meters by large antennas. The only effective way to send wireless power over long distances is using either laser or Microwave. In cloudy weather conditions, Microwave works properly but lasers have the same problem that solar power does on earth [170]. One way to harvest more energy from the sun is solar clothing. The produced energy can be used to cool down and warm up the person who wears it, charge the smart phones, smart watches, and other personal devices. The transparent panel is one idea that can be implemented in both clothes and buildings. Transparent panels are thin film technology, which can be installed on high-rises, transparent bus shelters, etc.

\subsection{Summary}

In this chapter a comprehensive review on challenges and developments in grid connected photovoltaic systems is provided. Many international and North American organizations such as 1547.8 group and IEEE 1547.7 are defining modeling recommendations and methodologies for renewable energy interconnection. Photovoltaic inverter manufacturers, utilities, and other involved area experts are focused on designing improved smart control strategies for PV inverters. However, there are still numerous gaps in the dynamic analysis of grid-connected PV performance. The increase of large scale PV-penetration circumstances needs a more thorough investigation of these gaps and systematic interconnection studies. Several technical challenges and required fundamentals for implementation of renewable energies in the grid was provided in this study. Secure, safe, 
and economic proper operation of the power network at voltage and frequency violations for different countries are specified by the grid codes. Inverters can be considered as the brain of a PV system. The inverter topologies are classified based on the number of power processing steps, the position of capacitors for decoupling the power, if they utilize transformers or not, and kinds of grid interface. Moreover, many control methods have been suggested by researchers to control the MPPT, current, voltage, active and reactive power. An overview of these controllers was discussed in this chapter. Finally, higher solar integration requires implementation of battery (and super-capacitor) energy storage systems to compensate high energy (and high power) fluctuations caused by stochastic nature of renewable resources. A brief description of energy storage systems as a whole, problems that are presented with the large scale integration of PV renewable energy, and the uses of energy storage systems to provide a means to resolve those problems were similarly discussed in this chapter. 


\section{CHAPTER 3}

\section{ANALYSIS OF CARBON TAX AS AN INCENTIVE TOWARD BUILDING SUSTAINABLE GRID WITH RENEWABLE ENERGY UTILIZATION}

\subsection{Overview}

This study presents an analysis of technical and financial viability of hybrid grid/Renewable Energy System (RES) configurations for a neighborhood in Miami. Assessment criteria comprised a reduction in net present cost (NPC), cost of energy (COE) and greenhouse

gases (GHG). Matlab ${ }^{\mathrm{R}}$ Simulink and the RES software HOMER were utilized as the assessment tool. The modeling is performed based on hourly load data of Miami, south-east coast of the USA. Grid connection is required to provide the energy back up and as well to commercialize the system. This research analyses cost efficacy to encourage the use of renewable energies, specifically, wind and solar. For this purpose, an evaluation of the Co2 tax level of the cost-effectiveness of the system is also conducted. Outcomes demonstrate that there is the remarkable potential of $\mathrm{Co} 2$ mitigation along with $\mathrm{COE}$ reduction and sustainable and resilient energy development from employing RES. The results show that there are enough prospects for renewable-based DG generation in existence of appropriate policy allocation for GHG emission penalties and Market Price Referent (MPR). There is a considerable quantity of literature available on renewable incentives. The focus of the experimental studies is mostly on the comparison of the different supporting action plans and to their advantage to promote the utilization of renewable technologies but not on their cost to reduce $\mathrm{Co} 2$ emissions. 


\subsection{Problem Statement}

Since most of the electrical energies are generated from fossil fuels, power generation becomes the leading source of greenhouse gases emission. This has led to severe environmental problems and concern of global warming. The Kyoto Protocol was effective on February 16, 2005, and most industrialized countries had to obey and endeavor to reduce their overall emissions of greenhouse gases [171]. Furthermore, the need of the tourism industry to adopt effective energy management strategies for reducing its impact on the environment and reduction of greenhouse gas (GHG) emissions has been recognized by international organizations [172]. The tourism locations are under ever increasing pressure to be called as green and this prospect is often connected with local sustainability agendas [173].

Nema et al [174] talk through sizing of Wind Turbine Generator (WTG), SPV arrays and other components for a SPV-Wind-battery-converter along with generator based power supply by simulation in HOMER (A public domain software developed by National Renewable Energy Laboratory, US).

Exploitation of certain renewable technologies, such as wind turbines and solar panels depends on the availability of renewable resource and as a consequence to augment the DGs during low power periods, energy grid connection or storage devices are used [175177].

In 2005, DeNA Co. distributed a widespread survey on wind deployment in Germany [178]. In addition to other findings, it evaluated the cost of Co2 diminution by reason of wind energy taking into account the cost of feed-in tariff (FIT). It compares the net cost and Co2 emissions of the system in 2007, 2010 and 2015 amongst two scenarios: At first study, it was considered that the future wind capacity remains equal to 2003, and the second one with a large wind capacity that is matured through the FIT. Consequences rely 
on the hypotheses made for the fuel and carbon prices. With a carbon price in the range of 5-10 per ton of $\mathrm{Co} 2$, the expected annual Co2 diminution cost of wind in the years 2007, 2010 goes from a minimum of $€ 56.6 / \mathrm{tCo} 2$ to a maximum of $€ 168.0 / \mathrm{tCo} 2$. All of these studies based on forecasts [179-181].

An optimization algorithm was proposed in [182] to join in two DGs and a microgrid into a Virtual Power Plant, having a number of loads, which will be able to produce and sell electric energy to load and electrical power market. Other references focused on technical issues of the penetration of RES into the grid. Because of the amplified usage of RES in power system, the fault ride-through operation and control of the DFIG wind power system and PV have become a research emphasis [171,183-186].

\subsection{Solution Approach}

HOMER is a time-step simulator that utilizes hourly load and environmental data inputs to assess the technical potential of RES via renewable fraction (RF) and economic viability via net present cost (NPC). HOMER also predicts the optimized RES configuration for a given set of constraints and sensitivity variables, based on NPC. Although simulation times can be lengthy, depending on the number of variables used (up to $48 \mathrm{~h}$ on a standard PC for some hydrogen storage configurations), its operation is simple and straightforward.

In this chapter, a case-study analysis was conducted for a neighborhood in Miami, the USA which is one of the most important tourist locations in the world. In the remaining part of the chapter, Section II reports formulas needed for modeling the system and calculation methods that are used by the software. Section III describes in detail all input data and their implementation in the simulation. Section IV provides a classification and general discussion of the costs and cost savings associated with the use of wind and 
solar energy. Section V presents the results and sensitivity analysis. Section VI gives conclusions.

\subsection{Modeling the Proposed System}

HOMER models each individual system configuration by performing an hourly time-step simulation of its operation for a one-year duration. The available renewable power is calculated and is compared to the required electrical load. Where the RES system is assessed as satisfying demand, any excess electricity is then spread to other secondary demands. Where demand is not assessed as satisfied, an alternative supply, either by diesel or grid generation, is sought to fill the deficit. While HOMER's 1-h time step is small enough to capture most of the statistical variability of the load and fluctuating renewable resources, it does not slow computation excessively.

Following calculations of one-year duration, any constraints on the system imposed by the user are then assessed; e.g. the fraction of the total electrical demand served or the proportion of power generated by renewable sources.

The three criteria used for the feasibility analysis of the RES were comprised net present cost (NPC), cost of energy (COE) and Greenhouse Gases (GHG) reduction. The projected lifespan was taken as 20 years.

The total net present cost of a system is the present value of all the costs that it incurs over its lifetime, minus the present value of all the revenue that it earns over its lifetime. Costs include capital costs, replacement costs, O\&M costs, fuel costs, emissions penalties, and the costs of purchasing power from the grid. Revenues include salvage value and grid sales revenue. HOMER calculates the total net present cost using the following equation: 


$$
C_{N P C}=\frac{C_{a n n, t o t}}{C R F(i, N)}
$$

Where $C_{a n n, t o t}$ is the total annualized cost (\$) (which is the sum of the annualized costs of each system component). The capital recovery factor (CRF) is given by:

$$
C R F(i, N)=\frac{i(1+i)^{N}}{(1+i)^{N}-1}
$$

HOMER assumes that all prices escalate at the same rate, and applies an 'annual real interest rate' rather than a 'nominal interest rate'. All costs are therefore calculated in constant dollars. This method allows 'inflation' to be factored out of the analysis. The overall annual (real) interest rate (i) in the simulations was taken to be $6 \%$, which was applied over a project lifetime assessed as 20 years. NPC estimation in HOMER also takes into account salvage costs, which is the value remaining in a component of the power system at the end of the project lifetime. HOMER assumes linear depreciation of components, meaning that the salvage value of a component is directly proportional to its remaining life. It also assumes that the salvage value is based on the replacement cost rather than the initial capital cost. This is stated mathematically as:

$$
S(\$)=C_{\text {rep }} \frac{R_{\text {rem }}}{R_{\text {comp }}}
$$

Where $C_{r e p}$ is the replacement cost of the component (\$), Rrem is the remaining life of the component ( $\mathrm{t}$ ) and $R_{\text {comp }}$ is the lifetime of the component $(\mathrm{t})$. HOMER defines the levelized cost of energy (COE) as the average cost per kWh of useful electrical energy produced by the system. To calculate the COE, HOMER divides the annualized cost of producing electricity (the total annualized cost minus the cost of serving the thermal load) by the total useful electric energy production. The equation for the $\mathrm{COE}$ is as follows: 


$$
C O E=\frac{C_{a n n, t o t}}{E_{\text {prim }, A C}+E_{\text {prim }, D C}+E_{\text {grid,sales }}}
$$

Where $E_{\text {prim }, A C}$ is the AC primary load served $(\mathrm{kWh} / \mathrm{yr}), E_{\text {prim }, D C}$ is the DC primary load served $[\mathrm{kWh} / \mathrm{yr}]$ and $E_{\text {grid,sales }}$ is the total grid sales $[\mathrm{kWh} / \mathrm{yr}]$. The capacity shortage fraction (CSF) is the fraction of the total load plus operating reserve that the system fails to supply (i.e. allowable blackout). A CSF of $0 \%$ of the hourly load was chosen for all grid-connected simulations, as the grid can be considered as infinite capacity storage (blackouts are still possible, but for a very small percentage of the time). Simulations examining a renewable energy-only configuration had a $2 \% \mathrm{CSF}$.

The operating reserve constraint (commonly called spinning reserve) is the additional reserve capacity required for a system to account for sudden increases in the electric load or sudden decreases in the renewable power output. It was set at $10 \%$ as recommended by Cotrell and Pratt [2]. Higher reserves were specified when using the renewable output. The higher reserve is required due to the inherent variability in the RES output and was set at $50 \%$ for PV and $50 \%$ for WECS.

\subsection{Data Inputs}

The load data was gathered through Florida Power \& Light Company (FPL) End-Use Load. FPL supplies electric service in most of the territory along the east coast of Florida (except the Jacksonville area and five other municipalities which have municipal electric systems), the agricultural area around southern and eastern Lake Okeechobee, the lower west coast area, and portions of central and north-central Florida. Energy consumption from 2004 to 2013 and forecast of rural electrical load demand in the FPL service territory is illustrated in Table 3.1. It is predicted that from 2014 to 2023 an increase of 13.2\% in an average number of customers and $12.7 \%$ in total consumption will occur. In addition, 
Table 3.1: History and Forecast of Energy Consumption and Number of Rural and Residential Customers [2]

\begin{tabular}{|c|c|c|c|c|}
\hline \multicolumn{5}{|c|}{ History of Energy Consumption } \\
\hline Year & Population & GWh & $\begin{array}{c}\text { Average } \\
\text { No. of } \\
\text { Customers }\end{array}$ & $\begin{array}{c}\text { Average } \\
\text { Consumption } \\
\text { (kWh/Customer) }\end{array}$ \\
\hline 2004 & $8,247,442$ & 52,502 & $3,744,915$ & 14,020 \\
\hline 2005 & $8,469,602$ & 54,348 & $3,828,374$ & 14,196 \\
\hline 2006 & $8,620,855$ & 54,570 & $3,906,267$ & 13,970 \\
\hline 2007 & $8,729,806$ & 55,138 & $3,981,451$ & 13,849 \\
\hline 2008 & $8,771,694$ & 53,229 & $3,992,257$ & 13,333 \\
\hline 2009 & $8,732,591$ & 53,950 & $3,984,490$ & 13,540 \\
\hline 2010 & $8,762,399$ & 56,343 & $4,004,366$ & 14,070 \\
\hline 2011 & $8,860,158$ & 54,642 & $4,026,760$ & 13,570 \\
\hline 2012 & $8,948,850$ & 53,434 & $4,052,174$ & 13,187 \\
\hline 2013 & $9,025,275$ & 53,930 & $4,097,172$ & 13,163 \\
\hline \hline \multicolumn{5}{|c|}{ Forecast of Energy Consumption } \\
\hline 2014 & $9,111,384$ & 55,739 & $4,141,538$ & 13,458 \\
\hline 2015 & $9,302,665$ & 57,047 & $4,228,484$ & 13,491 \\
\hline 2016 & $9,437,042$ & 58,097 & $4,289,564$ & 13,544 \\
\hline 2017 & $9,571,922$ & 58,693 & $4,350,874$ & 13,490 \\
\hline 2018 & $9,705,104$ & 59,404 & $4,411,411$ & 13,466 \\
\hline 2019 & $9,835,541$ & 60,036 & $4,470,700$ & 13,429 \\
\hline 2020 & $9,961,263$ & 60,791 & $4,527,847$ & 13,426 \\
\hline 2021 & $10,079,425$ & 61,219 & $4,581,557$ & 13,362 \\
\hline 2022 & $10,198,087$ & 61,929 & $4,635,494$ & 13,360 \\
\hline 2023 & $10,318,293$ & 62,870 & $4,690,133$ & 13,405 \\
\hline
\end{tabular}

the average annual electricity consumption for a Florida residential FPL customer was $13,163 \mathrm{kWh}$ in 2013 . As a result, load data were scaled to an average of $3.606 \mathrm{MWh}$ per day to meet a neighborhood of 100 houses energy needs. Annual electric demand is shown in Fig. 3.1. The annual energy consumption in the neighborhood is $1,316,196$ $\mathrm{kWh} / \mathrm{yr}$.

The coordinates of Miami were used in HOMER, $25^{\circ} 78 \mathrm{~N}$ latitude and $80^{\circ} 22 \mathrm{~W}$ longitude, to obtain monthly solar radiation values for Miami from NASA. Monthly average values of solar data are shown in Fig. 3.2. The solar annual average is $5.05 \mathrm{~kW} / \mathrm{m}^{2} / \mathrm{d}$. Clearness index is a dimensionless number between 0 and 1 , defined as the surface radiation divided by the extraterrestrial radiation. 


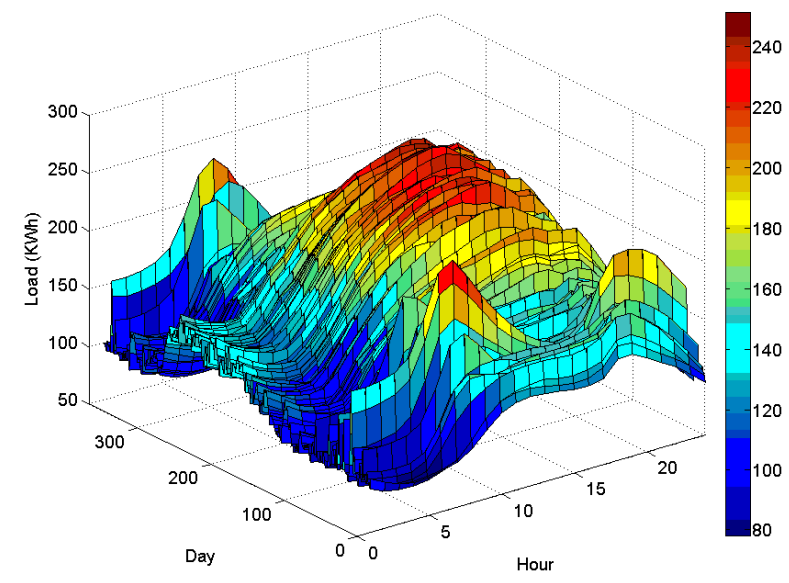

Figure 3.1: Annual Electric Demand of a Neighborhood in Miami.

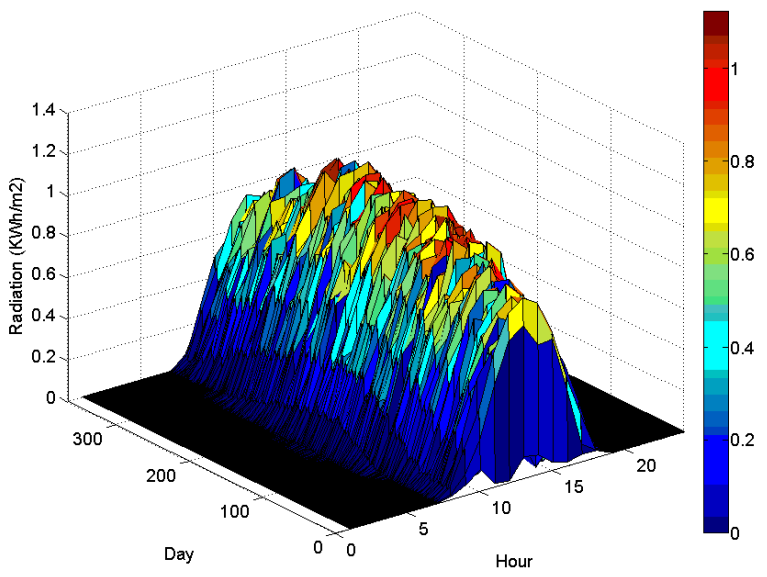

Figure 3.2: Hourly Solar Irradiation.

The clearness index has a high value under clear, sunny conditions, and a low value under cloudy conditions. The average clearness index is 0.558 .

In this study, wind speed data were taken out from NREL. These values are of $40 \mathrm{~m}$ height and thats why it was considered that anemometer height in HOMER simulations is $40 \mathrm{~m}$. The average wind speed for each month was entered and then Homer makes a wind profile by using Weibull $\mathrm{K}$ factor (a measure of the distribution of wind speed over a year) of 2 and autocorrelation factor (randomness in wind speed from hour-to-hour) of 


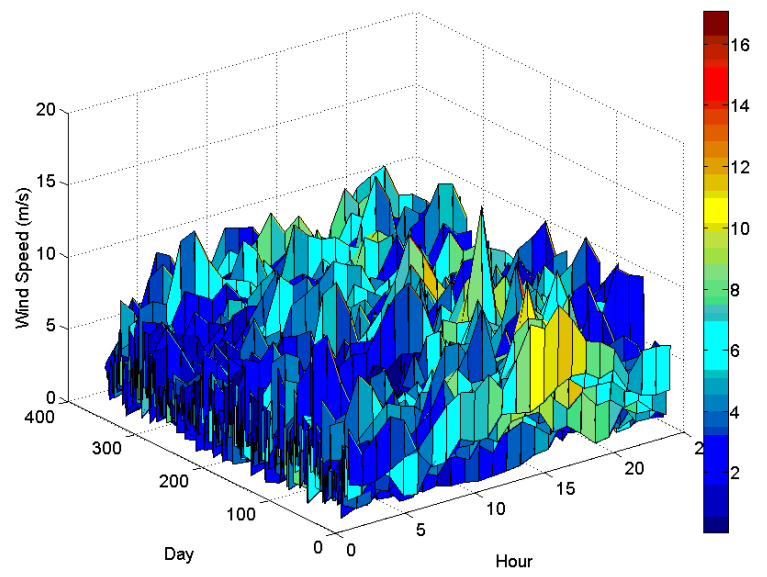

Figure 3.3: Hourly wind speed data.

0.85. The monthly averages wind speed is shown in Fig. 3.

\subsection{Description of the System}

The main components of the system are GE 1.5 sl wind turbine, electric grid, converter, and the loads. The capital cost, cost of operation, maintenance, and other costs contribute to the total cost of the system. In this study, two different DG technologies have been considered.

\subsubsection{Wind Turbine}

The capital cost of the wind turbine is generally ranged between $\$ 900$ and $\$ 1000$ per $\mathrm{kW}$. The replacement cost is about $15 \% 20 \%$ of the original cost and the O\&M cost is about $2 \%$ of the original cost $[173,187]$. A $1.5 \mathrm{MW}$ General Electric wind turbine was modeled in HOMER, meanwhile, a doubly fed induction Generator model of a 1.5-MW wind turbine

connected to a $25 \mathrm{kV}$ feeder by Matlab ${ }^{\circledR}$ Simulink was used to simulate the technical practicability of the system. 


\subsubsection{Photovoltaic panel}

The price of the PV panel is highly dependent on several factors which include the vendor, size of the panel, technology, and the trademark. In this study, SunPower modules (SPR$305)$ are used for $500 \mathrm{~kW}(5 \times 100 \mathrm{~kW}) \mathrm{PV}$ array. Each module of this array has an opencircuit voltage of $64.2 \mathrm{~V}$ and short circuit current of 5.96A.

\$945 as the capital cost of the PV [173] and \$95/kW as the O\&M cost were used as PV inputs in HOMER model. Detailed model of Matlab Simulink grid connected SunPower SPR-305-WHT-PV array was used for applying an assessment of the system.

\subsubsection{Electric Grid}

The system is grid-connected and therefore a part of the load is considered to be supplied by the grid. The cost of buying power from the grid is selected to be $\$ 0.070 / \mathrm{kW}$ and sell back rate was selected $0.129 \$ / \mathrm{kWh}$ based on [173]. It was also assumed that emission factors of Co2, So2 and Nox are $632 \mathrm{~g} / \mathrm{KWh}, 2.74 \mathrm{~g} / \mathrm{KWh}$ and $1.34 \mathrm{~g} / \mathrm{KWh}$ respectively. In addition, four levels of $\mathrm{Co} 2$ taxes ( $\$ 0, \$ 20, \$ 40$ and $\$ 60$ per ton of Co2 emission) are also considered for GHG emissions of the grid [2].

\subsubsection{Converter}

Note The PV panels supply DC power which needs to be connected to an AC load or grid. The cost of a power conversion system (PCS) is about $\$ 100$ per $\mathrm{kW}$. The cost summary of the system is represented in Table 3.2. The HOMER simulation results show that the addition of GE $1.5 \mathrm{sl}$ wind turbines is technically viable and economically profitable. However as urban land space is limited in Miami, only one wind turbines and 500Kw of PV panels are considere The cost summary of the system is represented in Table II. The 
Table 3.2: Cost Summary of the System

\begin{tabular}{|c|c|c|c|}
\hline Component & Capital (\$) & O\&M (\$) & Total (\$) \\
\hline PV & 472,917 & 549,601 & $1,022,517$ \\
\hline GE 1.5sl & $1,500,000$ & 344,098 & $1,844,098$ \\
\hline Grid & 0 & $-2,480,628$ & $-2,480,628$ \\
\hline Converter & 50,000 & 57,350 & 107,350 \\
\hline System & $2,022,917$ & $-2,198,447$ & $-175,530$ \\
\hline
\end{tabular}

Table 3.3: Variation of NPC and COE by Tax on Co2 for Two Scenarios

\begin{tabular}{|c|c|c|c|c|}
\hline \multirow{2}{*}{$\begin{array}{l}\text { Co2 } \\
\text { Tax }\end{array}$} & \multicolumn{2}{|c|}{ NPC (\$) } & \multicolumn{2}{|c|}{ COE (\$/kWh) } \\
\hline & WT & $\mathbf{W T}+\mathbf{P V}$ & WT & $\mathbf{W T}+\mathbf{P V}$ \\
\hline 60 & $-201,397$ & 49,620 & -0.016 & -0.014 \\
\hline 40 & $-70,605$ & 47,427 & -0.006 & 0.004 \\
\hline 20 & 60,187 & 268,940 & 0.005 & 0.021 \\
\hline 0 & 190,978 & 488,193 & 0.015 & 0.039 \\
\hline
\end{tabular}

HOMER simulation results show that the addition of GE $1.5 \mathrm{sl}$ wind turbines is technically viable and economically profitable. However as urban land space is limited in Miami, only one wind turbines and $500 \mathrm{Kw}$ of PV panels are considered. Optimized converter size of this configuration was calculated as $300 \mathrm{kw}$.

\subsection{Simulation Results}

The Matlab Simulink and HOMER model of the investigated systems are given in Fig. 3.4. For the HOMER simulation, two different cases are considered which are as listed below:

- Wind Turbine System

- Hybrid Wind Turbine and PV System

Variation of net present values and leveled cost of energy (COE) of different cases with carbon taxes is presented in Table 3.3. Increasing carbon taxes from $\$ 0$ to $\$ 60$ per ton of $\mathrm{Co} 2$ in both Wind turbine and hybrid wind turbine and PV systems decreases the COE from $\$ 0.015$ to $\$-0.016$ and from $\$ 0.039$ to $\$-0.014$ per $\mathrm{kWh}$ respectively. 


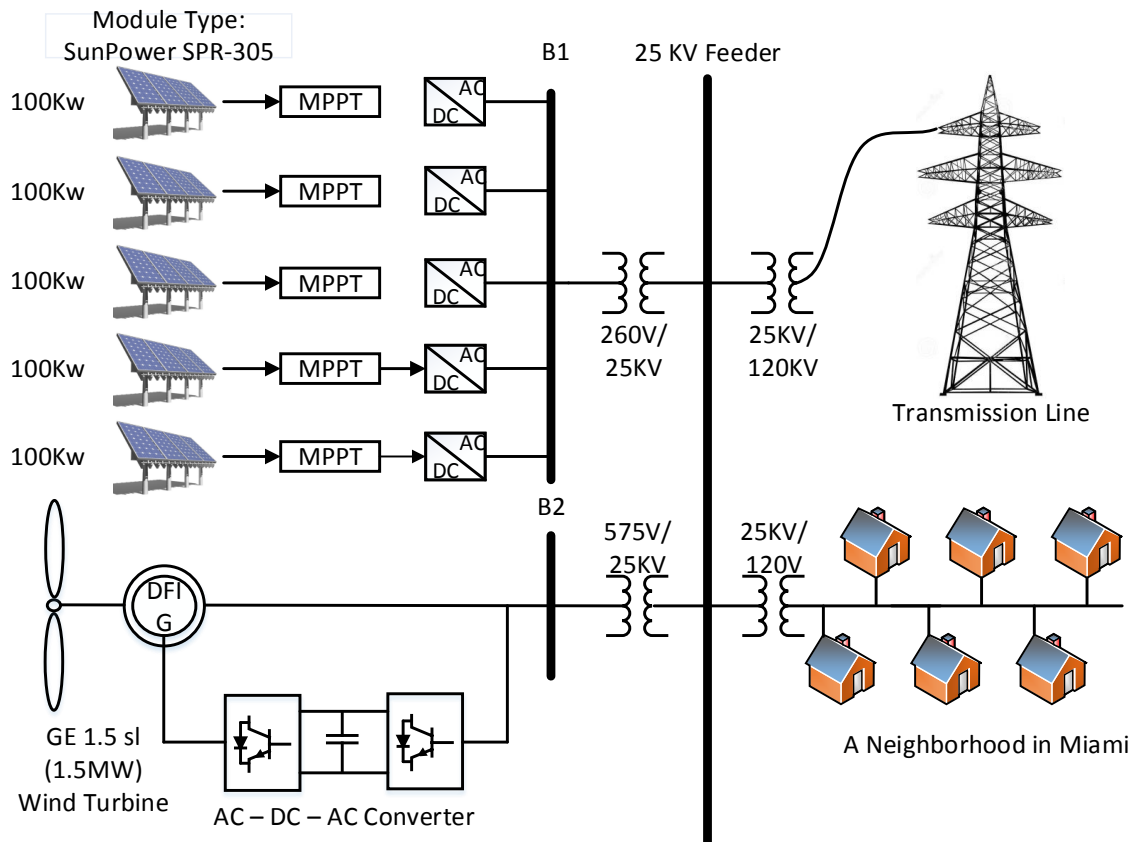

Figure 3.4: Simulated Model used in Matlab Simulink and HOMER software.

Table 3.4: GHG Emissions for Two Scenarios

\begin{tabular}{|c|c|c|}
\hline Pollutant & $\begin{array}{c}\text { Emissions (Kg/yr) } \\
\text { (WT) }\end{array}$ & $\begin{array}{c}\text { Emissions (Kg/yr) } \\
\text { (WT + PV) }\end{array}$ \\
\hline Carbon Dioxide & $-630,433$ & $-1,075,281$ \\
\hline Sulfur Dioxide & $-2,733$ & $-4,662$ \\
\hline Nitrogen Oxides & $-1,337$ & $-2,280$ \\
\hline
\end{tabular}

Though carbon tax helps, primarily, in mitigating Co2 emissions, there are also secondary environmental benefits in terms of So2 and Nox emissions reductions (see Table table:table4 ${ }^{*}$ carbon). So 2 and Nox emissions fall because the carbon tax leads to an overall reduction in thermal-based electricity generation. So2 and Nox emissions decrease at the same rate as $\mathrm{Co} 2$ does at higher tax rates.

Since the system sells more power to the grid than it buys from the grid over the year, the net grid purchases will be negative and so will the grid-related emissions of each pollutant.

Monthly average electric productions of each component for both scenarios are shown in Fig. 3.5 and 3.6 respectively. It is seen that around $82 \%$ of total annual load is supplied 


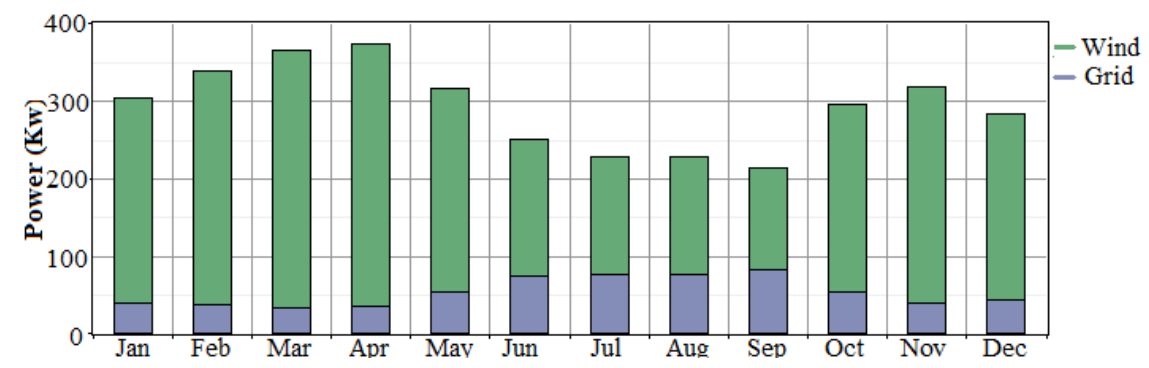

Figure 3.5: Average Electric Power Production for Wind Turbine System.

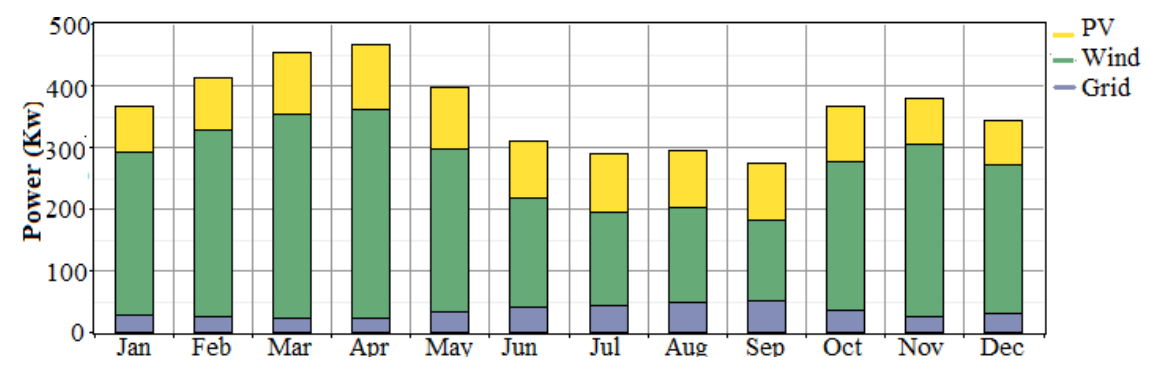

Figure 3.6: Average Electric Power Production for Hybrid Wind Turbine and PV System.

by Wind turbines for the first scenario and about $25 \%$ by PV panels and $66 \%$ by Wind Turbines for the second scenario.

Furthermore, a comparison between Matlab Simulink model and HOMER model active power output results of the wind turbine is performed and the result is illustrated in Fig.3.7. The wind turbine power output is shown for 16th to 22nd of March as 20th of the March had the highest wind speed during the year. There are differences between these two simulations because in the Simulink model the inertia constant and friction factor of turbine and generator was considered, but as it can be seen both models are technically viable. Also, other measurements of wind turbine system like Bus B2 voltage, active and reactive power, $\mathrm{DC}$ bus voltage of $\mathrm{AC} / \mathrm{DC} / \mathrm{AC}$ converter and wind speed pattern are shown in Fig. 3.8. As the wind turbine operates in a voltage regulation mode the voltage at grid terminals is kept constant by a voltage regulator. The output of the voltage regulator is the d-axis reference current that must be injected into the rotor by the rotor side converter. Generally speaking voltage at bus B2 is regulated by generation or absorption 


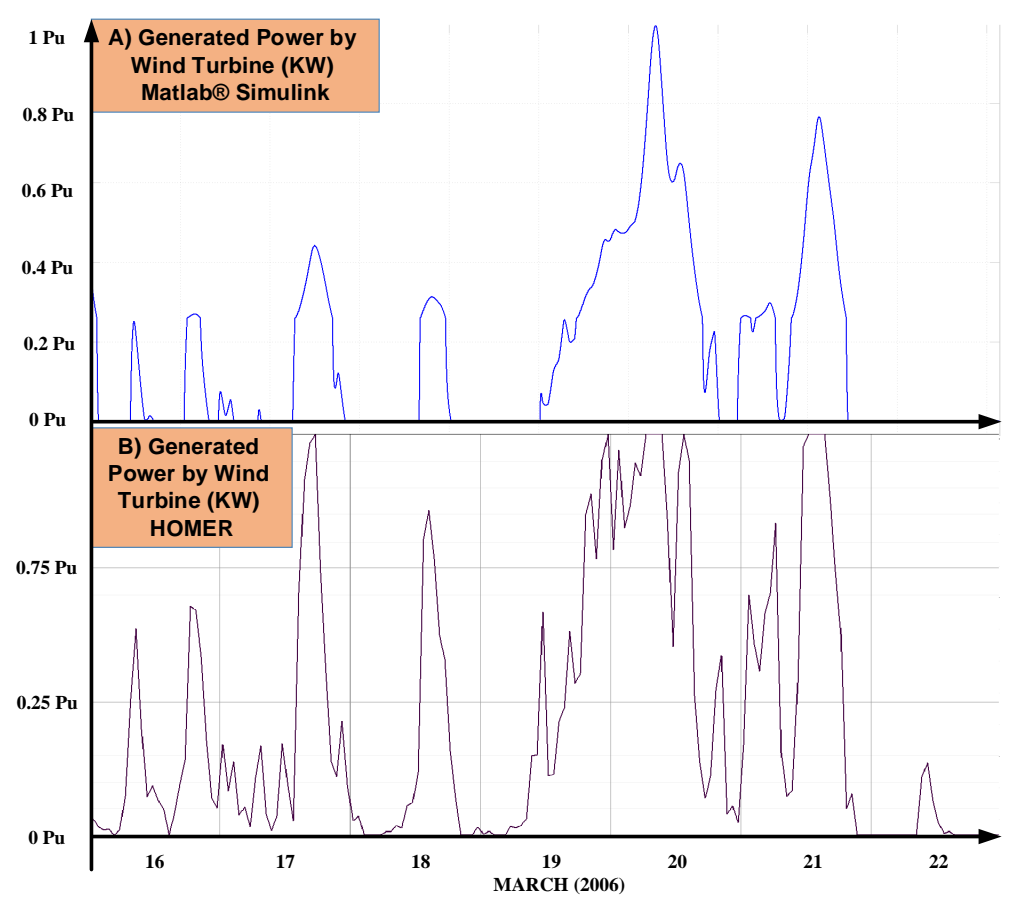

Figure 3.7: Comparison of power generated by wind turbine in A) Matlab Simulink and B) HOMER.

of reactive power at the grid connection point. Moreover, DC link voltage is regulated by the grid side converter. There are a lot of details in the control of the Doubly Fed Induction Generator Wind Turbine and also in Maximum Power Point Tracker of PV system which is beyond the scope of this study.

In another comparison difference of active power generated by the PV panel on July 11, which has the most irradiance during the year, in different models is presented in Fig. 3.9. For the reason that a Maximum Power Point Tracking system is used by the PV model in Matlab Simulink, but it was not used in HOMER model and also the effect of slope of panels and clearness index are not considered by Simulink, the generated active power which is simulated by HOMER is about $30 \%$ less than the active power in Simulink.

Though average of wind speed is low in Miami, Simulation results reveal that implementing a grid-connected WT or WT+PV would be cost-effective even without considering Co2 taxes (comparing to $0.070 \$ / \mathrm{KWh}$ for grid electricity price). Moreover, by 


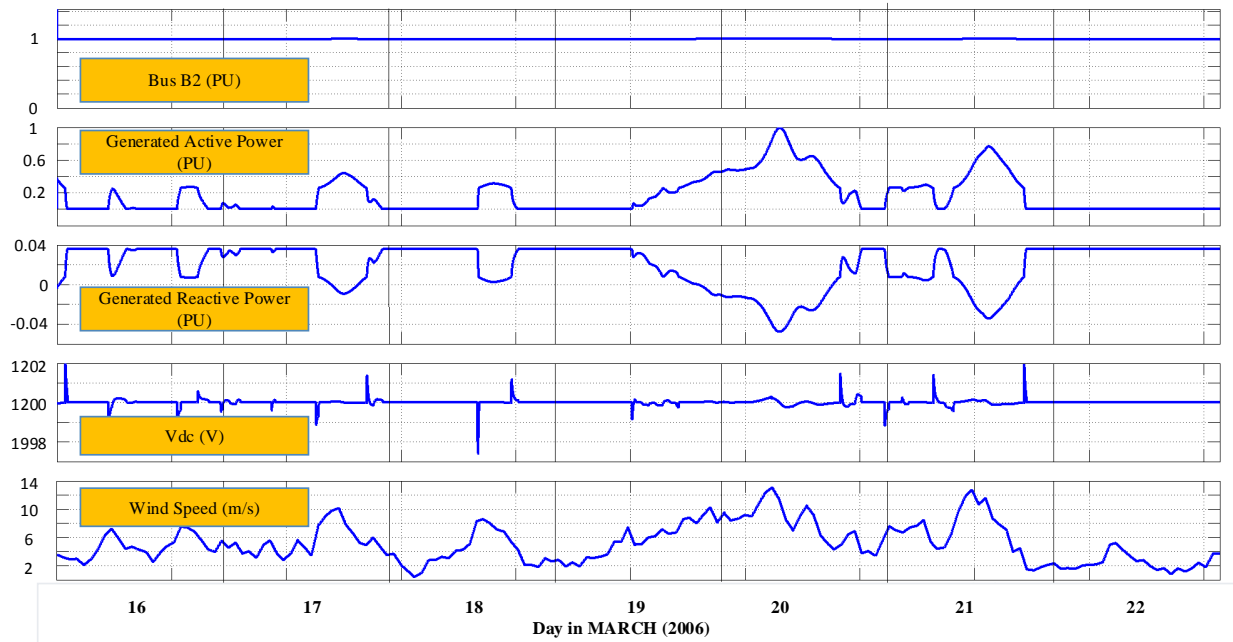

Figure 3.8: Bus B2 voltage, active and reactive power, DC bus voltage of AC/DC/AC converter and wind speed pattern.

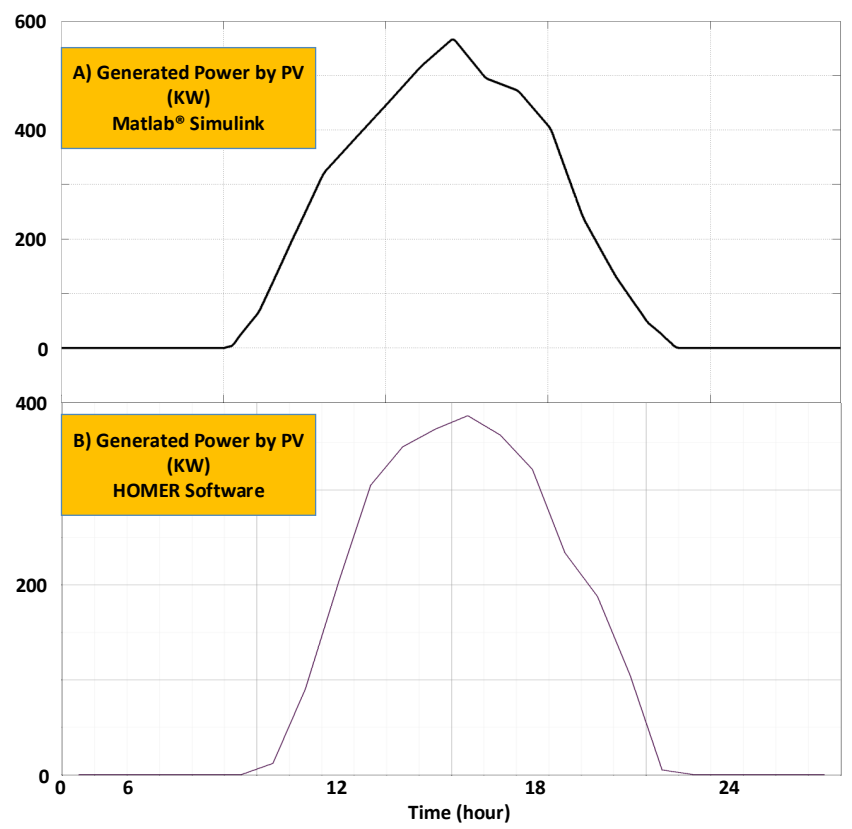

Figure 3.9: Comparison of power generated by Photovoltaic Panels in A) Matlab Simulink and B) HOMER.

imposing a $\mathrm{Co} 2$ tax, cost of energy is negative when the $\mathrm{Co} 2$ tax is increased. Negative numbers in table 3.2 can be interpreted as not only customers do not pay for electricity but also they can make money by implementing RES. 
Considering mitigation of environmental pollutant gases (TABLE IV), NPC and COE (TABLE 3.3) and the need of the tourism industry to be called green, RES could be starting to establish apace and more research should be done to develop the utilization of renewable energies to 20 percent by 2020 . These goals are reachable by doing the strongly advised proposal:

- Reducing GHG emission and using the Clean Development Mechanism (CDM) credits.

- Economic and environmental benefits.

- Developing travel industry

\subsection{Summary}

This chapter presents an analysis of carbon tax, as strategy option, for encouraging the use of renewable-based DGs and evaluate the cost-effectiveness of the system in terms of GHG mitigation potentials. Different scenarios include different types of RES and different penetration levels of the distributed generation. An economic study for the NPC with both WT and PV+WT was also conducted which shows that, through imposing appropriate taxes on Co2 and setting remunerative "buy back" prices by the government, there are remarkable potentials of $\mathrm{Co} 2$ mitigation along with $\mathrm{COE}$ reduction. Similarly, technical analysis was accompanied by both HOMER and Matlab Simulink to show that the proposed Renewable energy system can be used in the real world.

In summary, there are enough prospects for renewable-based DG generation in existence of appropriate policy allocation for GHG emission penalties and Market Price Referent (MPR), as well as technical measures, in order them to be competitive with the grid and therefore keep away from environmental emissions from the centralized grid. It 
is suggested that further studies be performed on offshore wind turbines in combination with subsea transmission power supplies. 


\section{CHAPTER 4}

\section{ARTIFICIAL NEURAL NETWORK BASED DUTY CYCLE ESTIMATION FOR MAXIMUM POWER POINT TRACKING IN PHOTOVOLTAIC SYSTEM}

\subsection{Overview}

According to a nonlinear current-voltage characteristic of Photovoltaics (PV) we need to track maximum power output of PV generation units instantly. The aim of chapter 4 is to introduce a non-complicated method for tracking the maximum Power Point without any previous knowledge of the physical parameters linked with a Grid-Connected photovoltaic (PV) system using artificial neural networks (ANN) modeling. The ANN is trained in various conditions of PV Output Voltage and PV Output Current to forecast the Duty Cycle of DC-DC boost converter as the MPPT device. The proposed technique is implemented in Matlab/Simulink and compared with the conventional method of incremental conductance. Simulation results show a good performance of the ANN based MPPT controller. MPPT techniques that properly detect the global MPP has been widely investigated in the literature. They include hill climbing $(\mathrm{HC})$, incremental conductance (IncCond), perturb and-observe (P\&O), and fuzzy logic controller (FLC). As the best of our knowledge estimation of the duty cycle of the DC-DC boost converter by Artificial Neural Network and using it in place of the whole MPPT controller and using Voltage and current has not been done so far in the literature.

\subsection{Problem Statement}

Renewable energy sources of energy ,e.g. photovoltaic (PV), power play a essential role in electric power generation, and become important these days due to unavailability and ecological impacts of traditional fuels. In the future, PV energy will increase more rank 
due to the shortage of fossil fuels and their environmental effects. More than 45 percent of necessary energy in the world will be generated by PV arrays [188]. Unfortunately, PV systems have two main problems: the conversion efficiency of electric power generation is low, and the amount of electric power generated by solar arrays changes rapidly with weather conditions [184, 189]. Moreover, because of nonlinear IV and PV characteristics of PV systems, their output power is always changing with weather conditions, i.e., solar radiation, atmospheric temperature and also nature of load connected $[190,191]$. Maximum power point tracking (MPPT) is critical as there is a possible incompatibility between the load characteristics and the maximum power points (MPPs) of the PV module in order to make sure optimal exploitation of solar cells [192]. By means of MPPT the cost of energy generated by PV panels is reduced [193]. A large number of methods have been suggested for tracking the MPP of PV systems in recent years. There are many techniques available in the literature such as fractional opencircuit voltage and short-circuit current [188] and the fuzzy logic control [194]. Also tracking the maximum power by means of look-up table in the microcomputer was proposed in [195]. There are some other popular methods like incremental conductance method (INC)[196] and the hill climbing method (HC). These techniques are widely applied in the MPPT controllers due to their simplicity and easy implementation. Numerous different MPPT approaches have been proposed, but there has been no direct use of output voltage and current to calculate the Duty cycle of the MPPT switches. The objective of this work was to bridge this gap. In this work, the consideration will be focused on simulation comparison study between Incremental Conductance technique and ANN, network, which used the solar irradiance as Input signal to estimate the effects of random cloud movement on the electrical parameters of the MPPT and the variables of the inverter was proposed by Giraud and Salameh in [197].

Similarly, tracking the maximum power by implementing microcomputer with a lookup 


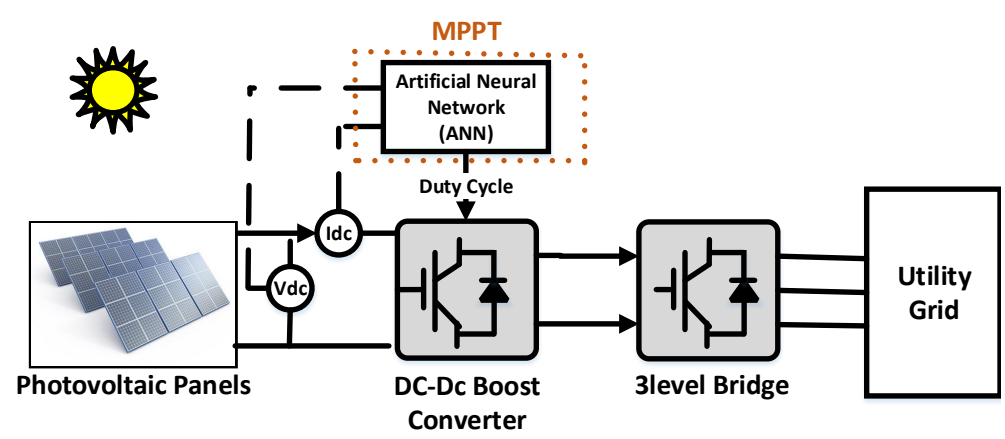

Figure 4.1: Block diagram of the configuration of the proposed system.

table was suggested in [198]. There are additional widely used methods like incremental conductance method (IncCond) [199] and the hill climbing method (HC). These techniques are widely applied in the MPPT controllers because of their clarity and easy application. However, to the best of our knowledge estimation of the duty cycle of the DC-DC boost converter by Artificial Neural Network and using it in place of the whole MPPT controller and using Voltage and current has not been done so far in the literature. The objective of this study is to bridge this gap. In this work, the attention will be focused on simulation assessment study between Incremental Conductance Technique and ANN, considering the panel output current and voltage variation in order to better performance in actual changing irradiance conditions.

\subsection{Solution Approach}

The formation of the proposed system consists of the PV array, Artificial Neural Network MPPT, DC-DC boost converter and 3level bridge inverter as shown in Fig. 4.1. Solar irradiance (E) in $W / m^{2}$ and the cell temperature (Tc) in degree Celsius are the inputs for the PV array, where the actual voltage and current expressed in $V_{d c}$ and $I_{d c}$.

The coordinates of the city of Miami in the USA were used in HOMER Software, $25^{\circ} 78 \mathrm{~N}$ latitude and $80^{\circ} 22 \mathrm{~W}$ longitude, to obtain hourly solar radiation values. Hourly 


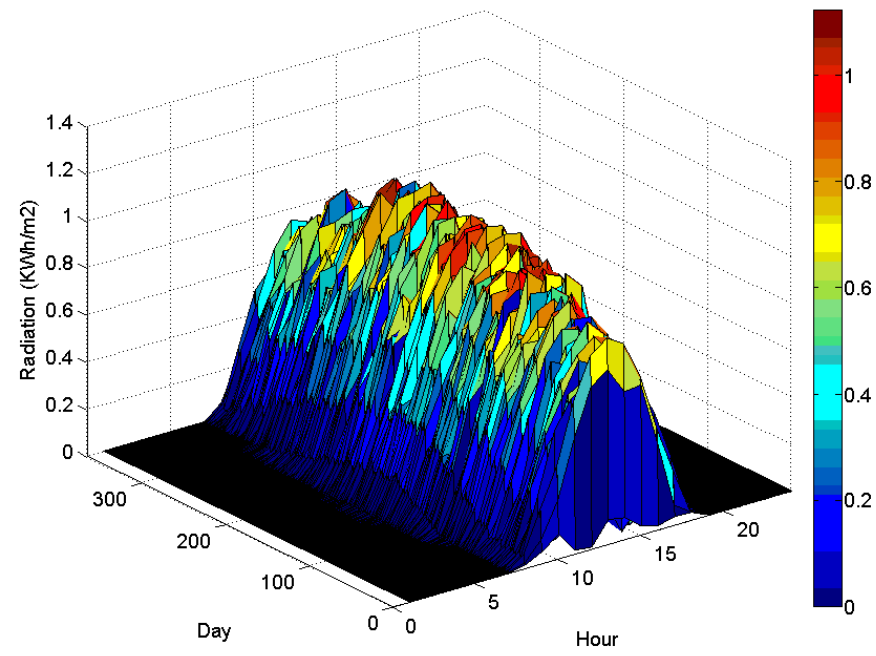

Figure 4.2: Hourly solar irradiation for one year.

average values of solar data are shown in Fig. 4.2. In this study the temperature was considered constant at $25^{\circ} \mathrm{C}$.

\subsubsection{PV array characteristics}

The Fig. 4.3 illustrates the equivalent circuit of the PV cell where $I_{p h}$ is current source of the PV array, largely depends on the insolation and cells temperature. $R_{s h}$ is an equivalent shunt resistance, $R_{s}$ is an equivalent series resistance, I and $\mathrm{V}$ are the output current and output voltage of the PV array. Generally, for uncomplicatedness $R_{s h}$ and $R_{s}$ are considered to be open circuit and short circuit, respectively. The shortened mathematical model of the output current and voltage is given as:

$$
I=n_{p} I_{p h}-n_{p} I_{r s}\left(e^{\frac{q}{p k n_{s}} \times \frac{V}{T}}-1\right)
$$

Where:

$I_{r s}:$ Cell reverse saturation current

$\mathrm{q}:$ Electronic charge 


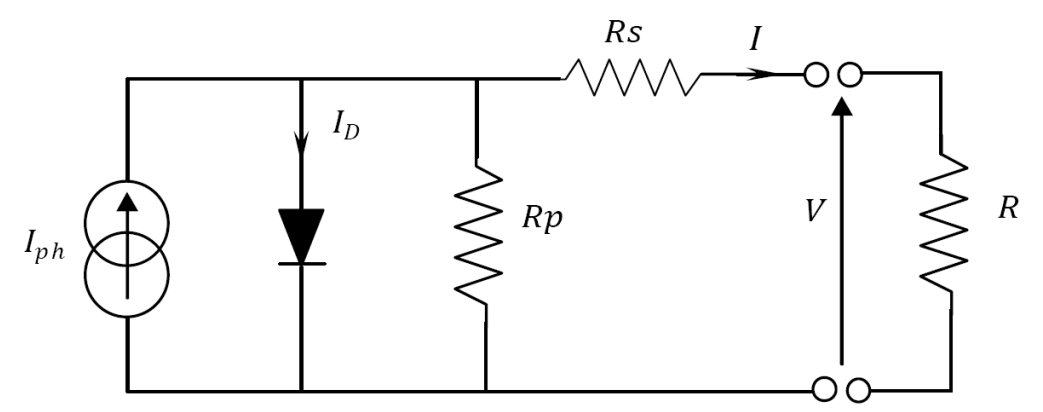

Figure 4.3: The equivalent circuit of a photovoltaic array.

$\mathrm{k}$ : Boltzmann's constant $\left(1.38 \times 10-23 \mathrm{~J} /{ }^{\circ} \mathrm{K}\right)$

$\mathrm{T}:$ Cell surface temperature $\left(\mathrm{K}^{\circ}\right)$

$\mathrm{p}:$ Cell ideality factor $(\mathrm{p}=15)$

$n_{p}:$ Number of solar cells in parallel

$n_{s}:$ Number of solar cells in series

The current source of PV array, $I_{p h}$ varied according to solar irradiation and cell temperature, is given by:

$$
I_{p h}=\left(I_{s c}+k_{I}\left(T-T_{r}\right)\right) \lambda / 100
$$

Where:

$T_{r}:$ Reference temperature;

$I_{s c}$ : Short circuit current at reference temperature and solar irradiation;

$K_{I}$ : Short circuit current temperature coefficient at reference temperature and solar irradiation;

$\lambda$ : Solar radiation, irradiation, or insulation $\left(W / m^{2}\right)$.

In this study we are going to develop an artificial neural network based MPPT controller for the PV arrays. In the incremental conductance method, which is used to gen- 
Table 4.1: Specification of Sunpower SPR-305 PV Module on Standard Test Conditions $\left(1000 \mathrm{~W} / \mathrm{M}^{2}, 25^{\circ} \mathrm{C}\right)$

\begin{tabular}{|l|l|}
\hline Maximum power & $305 \mathrm{~W}$ \\
\hline Open circuit voltage & $64.2 \mathrm{~V}$ \\
\hline Short circuit current & $5.96 \mathrm{~A}$ \\
\hline Voltage at maximum power point & $54.7 \mathrm{~V}$ \\
\hline Current at maximum power point & $5.58 \mathrm{~A}$ \\
\hline
\end{tabular}

erate training data for the Artificial Neural Network in this study, the controller senses incremental variations in current and voltage array to foresee the consequence of a voltage alteration. This method involves more calculation in the controller, but changing conditions can be tracked more quickly than perturb and observe method (P\&O). Similar to the $\mathrm{P} \& \mathrm{O}$ algorithm, it may produce swaying in output power.

In this chapter, a 100-kW PV array of 330 SunPower modules (SPR-305) is used for a Matlab simulation model. The array involves 66 parallel strings of 5 series-connected modules connected in parallel $(66 \times 5 \times 305.2 W=100.7 k W)$ [187]. The electrical specification of the mentioned module on standard test condition (STC) is shown in Table 4.1. I - V and P - V curves of single module at $25^{\circ} \mathrm{C}$ for different irradiance is illustrated in Fig. 4.4.

\subsubsection{Neural Network Architecture}

Lately, the use of ANN has entered various scientific areas as an approximation technique because of the very good pattern recognition capability [200]. A three-layer neural network can fairly perfectly estimate any nonlinear function to a random accuracy. A three layer feedforward backpropagation ANN is used: an input, a hidden and an output layer to guess Duty Cycle of DC-DC boost converter. The input layer consists of a two dimensional vector, one is the DC output Voltage of PV modules and the other is the PV current, 

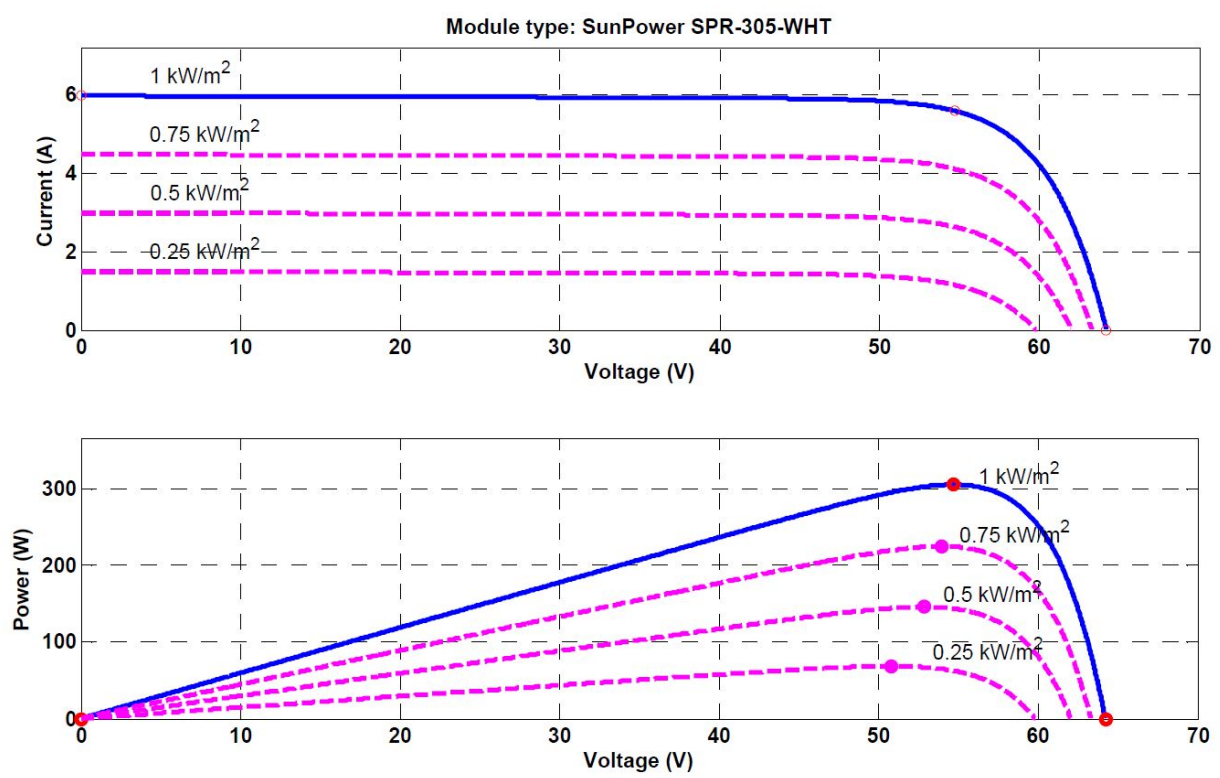

Figure 4.4: I-V and P-V curves of single module at $25^{\circ} \mathrm{C}$ for different irradiance

output layer is one dimensional vector consisting of Duty cycles. The training procedure needs a set of samples of appropriate network behavior inputs and target outputs.

The procedure of training a neural network includes modification of the weights and biases of the network to enhance network performance. Throughout the training, the connection weights are modified until the best fit is attained for the inputoutput patterns based on the minimum errors. The default performance function for feedforward networks is mean square error (MSE) which is the average squared error between the outputs, a, and the target outputs t. It is shown as:

$$
F=m s e=\frac{1}{N} \sum_{i=1} N\left(e_{i}\right)^{2}=\frac{1}{N} \sum_{i=1} N\left(t_{i}-a_{i}\right)^{2}
$$

Another performance function for neural networks is the mean absolute percentage error (MAPE), which is a measure of exactness of the method specifically in trend estimation. It typically articulates accuracy as a percentage, and is well-defined by the formula: 
Table 4.2: Training Parameter Values

\begin{tabular}{|l|l|}
\hline Number of Hidden Layers & 26 \\
\hline Epochs between displays & 5 \\
\hline Learning rate & 0.001 \\
\hline Maximum number of epochs to train & 1000 \\
\hline Performance goal & 0 \\
\hline
\end{tabular}

$$
M A P E=\frac{1}{N} \sum_{i=1}^{N}\left|\frac{t_{i}-a_{i}}{a_{i}}\right|
$$

In this chapter, we used MAPE as the evaluation factor of our approximation. Training parameter values of the proposed network are tabulated in Table 4.2.

\subsection{Results and Discussions}

In this study, quite a lot of inhomogeneous irradiance distributions are utilized to test the operation of the proposed scheme. In order to generate data that can be used as training sets of Artificial Neural Network we run the Simulink model with hourly average irradiance of the first 42 weeks of the year from 7 am in the morning to $5 \mathrm{pm}$ in the evening. As the control system uses a sampling time of 100 microseconds for voltage and current controllers, simulation of each day produces 20000 inputs of Voltage, Current and Duty Cycle which are big enough for training the network. In the simulation we used a time step of 0.1 for each hour and ran the simulation for 42 seconds. The simulation results for extracting the training data is shown in Fig. 4.5. The simulation was run by using the irradiance data of the last 10 weeks of the year to calculate the testing data of the neural network which are depicted in Fig. 4.6. As it can be seen from Figs 4.5 and 4.6 stated maximum power of $100.7 \mathrm{~kW}$ is obtained at times of a $1000 \mathrm{~W} / \mathrm{m}^{2}$ irradiance and generally power is tracking the irradiance, which means that the incremental conductance method can produce a reliable set of training and testing data. In the next step the acquired 

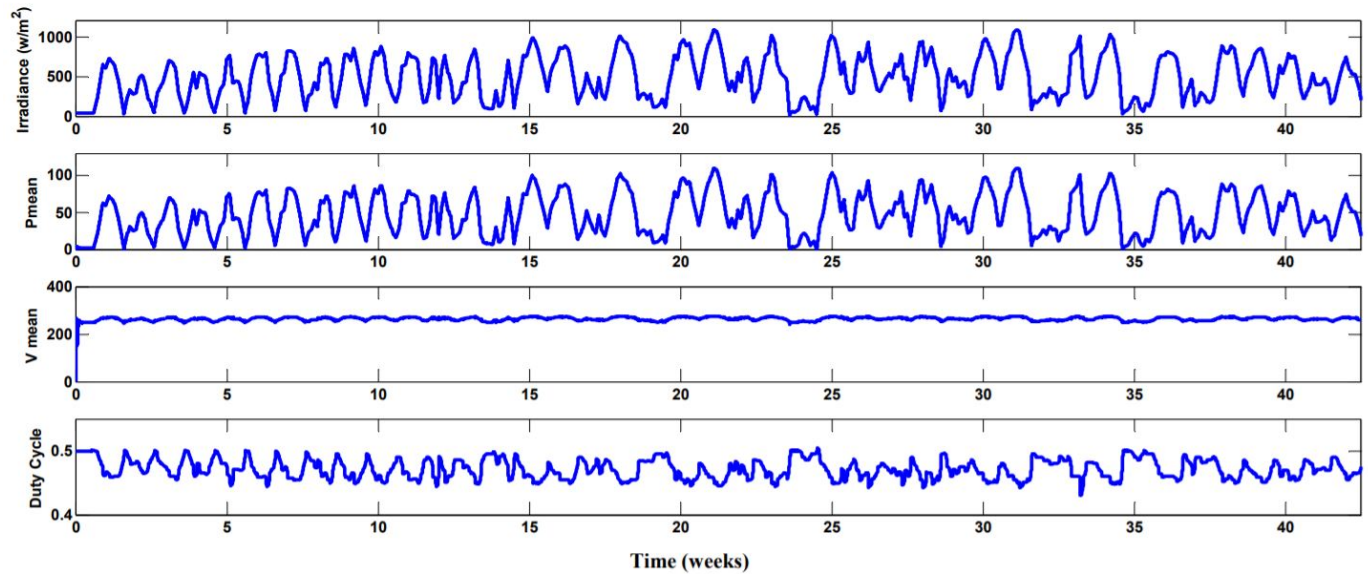

Figure 4.5: Irradiance, output voltage, output current duty cycle and generated power of PV system for the first 42 weeks of the year (Training Data)
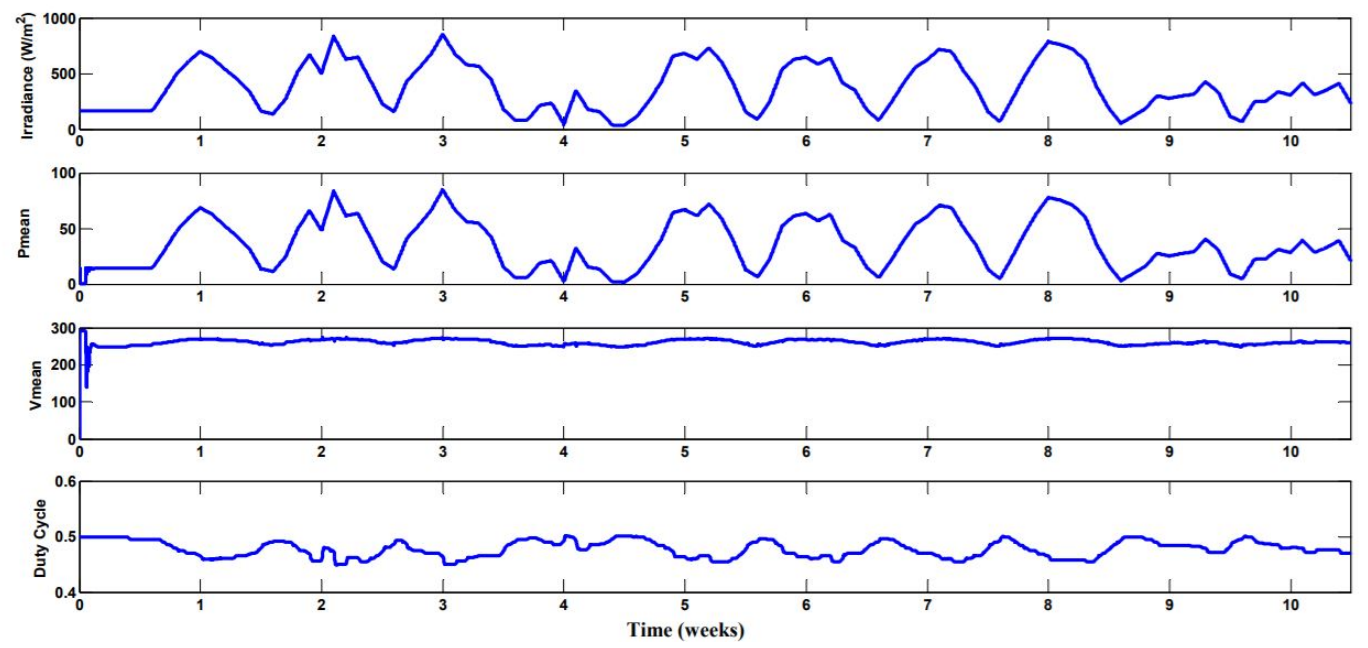

Figure 4.6: Irradiance, output voltage, output current duty cycle and generated power of PV system for the last 10 weeks of the year (Testing Data).

data are used to simulate the neural network to train and then approximate the duty cycle of the MPPT.

Fig. 4.7 shows the estimated (blue line) and actual (green line) values of the Duty Cycle ratio. Undoubtedly considering the mean absolute percentage error of $1.49 \%$ the results approved the effectiveness of the proposed technique.

The main advantages of the proposed MPPT method is that the system needs less 


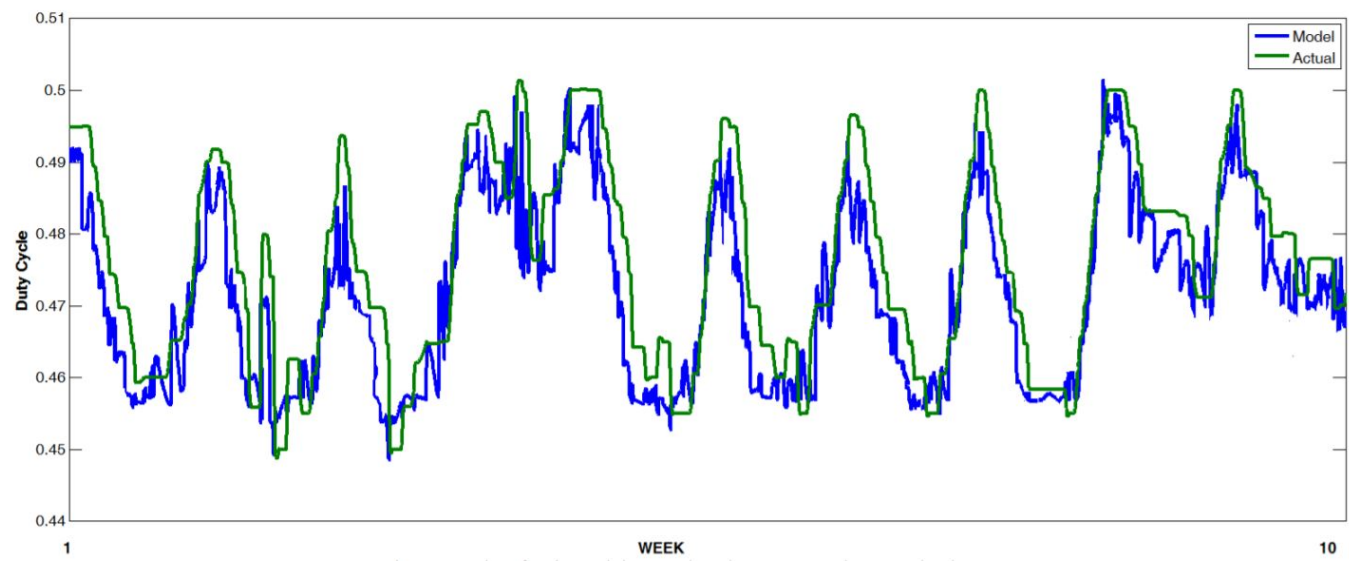

Figure 4.7: Results of estimated duty cycle values compared to actual values.

computational work because of no necessity for awareness of internal MPPT system parameters and the system offers a compressed solution for this multivariable problem.

\subsection{Summary}

The productivity of the suggested Artificial Neural Network structures for the MPPT control and the forecast of Duty Cycle of DC-DC boost converter has been presented. Since the duty cycle is directly achieved by using ANN, the proposed system does not need complicated processes and cutting-edge power electronic control units. The results how that the ANN is sufficiently accurate and can identify the duty cycle under different solar irradiance. 


\section{CHAPTER 5}

\section{ANALYZING THE TRENDS OF VOLTAGE FLUCTUATIONS, HARMONICS, FREQUENCY, AND LEGACY DEVICES OF THE PHOTOVOLTAIC TEST-BED}

\subsection{Overview}

In 1982, IEEE standard 519 was first released and then updated in 2014. It sets some rules and steady state limitations on Total Harmonic Distortion (THD) with reference to both current and voltage at the Point of Common Coupling (PCC). However, exceeding these boundaries may be encountered by transient conditions. IEEE Standard 1547 was published in 2003 and the latest amendment was issued in 2014. This standard contains power quality, response to unusual situations, and installation assessment. The specified requirements are essential for interconnection of distributed resources (DR), including power inverters/converters and synchronous generators. The standards are valid to all DR technologies, with combined capacity of 10 MVA or less at the PCC, connected to secondary and/or primary distribution voltages. This chapter presents monitoring and assessment of a 1.1MW photovoltaic power plant at Florida International University, Miami, Florida. The assessment criteria were the IEEE 519 and 1547. Revolution power quality recorder and AlsoEnergy power track software captured the harmonics and voltage data with resolution on 1 minute. It concluded that at current level of photovoltaic penetration (16\%), Current THDs violates the 5\% limit value at the Point of Common Coupling (PCC). Moreover, except from a few short duration voltage impulses, no voltage violation has been observed. 


\subsection{Problem Statement}

The proliferation photovoltaic $(\mathrm{PV})$ power generation systems in the distribution power grid motivate utilities to monitor the power quality (PQ) effects of these renewable power generators in the electrical networks. The power quality may cause voltage fluctuations and flicker, DC injection, harmonic emission, over voltages, and harmonics at the switching frequency $[8,9]$. Nowadays most companies rely on sophisticated equipment to be able to compete other competitors. This equipment could be affected some power quality issues which are undetectable by naked eyes. The benefits of high power quality of the power system are optimizing the reliability of the systems, early prediction of incipient problems, decreasing the energy expenditure, prevent power outages due to hidden disturbances, assurance of data integrity and reduced corrupt data in smart grids, etc. It is very difficult to quantify the power quality concerns since they are different by the interaction between susceptible equipment and power quality. When "good" power quality for one equipment could be "bad" for another one [10]. Therefore, the best assessment criteria are standards. In 1982, IEEE standard 519 was first released and then updated in 2014. It sets some rules and steady state limitations on Total Harmonic Distortion (THD) with reference to both current and voltage at the Point of Common Coupling (PCC). However, exceeding these boundaries may be encountered by transient conditions. IEEE Standard 1547 was published in 2003 and the latest amendment was issued in 2014. This standard contains power quality, response to unusual situations, and installation assessment. The specified requirements are essential for interconnection of distributed resources (DR), including power inverters/converters and synchronous generators. The standards are valid to all DR technologies, with combined capacity of 10 MVA or less at the PCC, connected to secondary and/or primary distribution voltages. The power quality concept has always been oversimplified and misunderstood, but due to a significant increase in the use of 


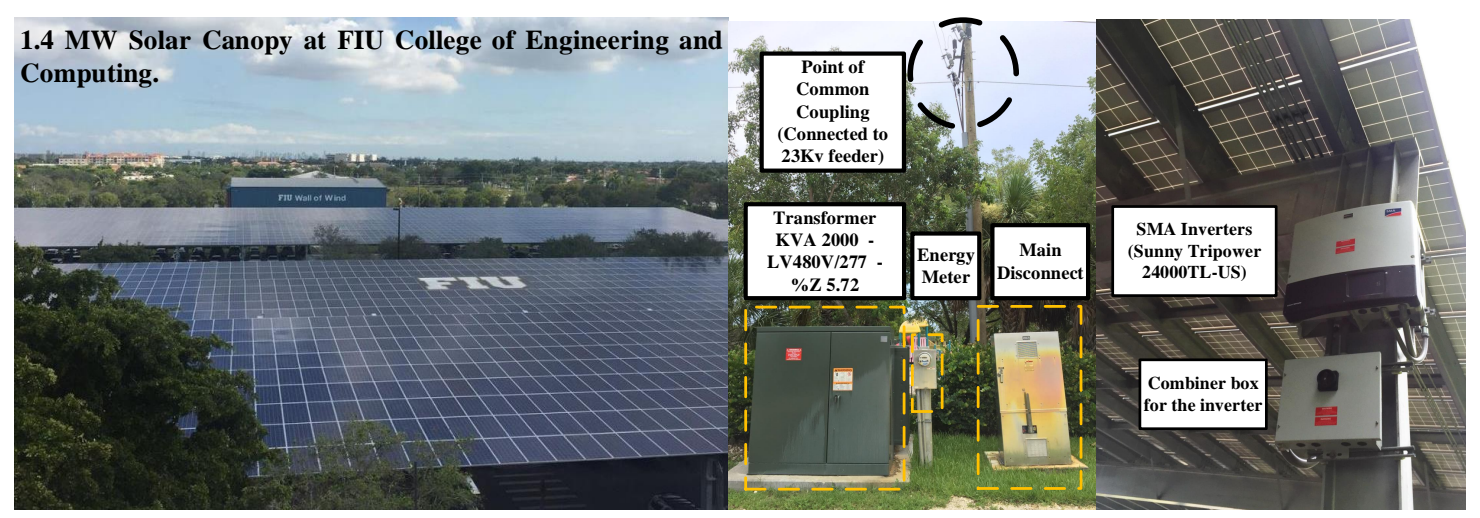

(a)

(b)

(c)

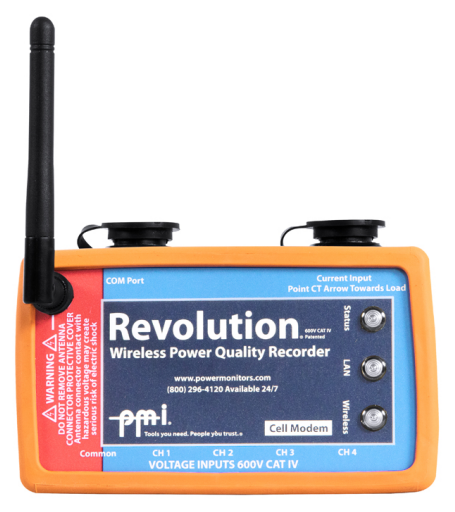

(d)

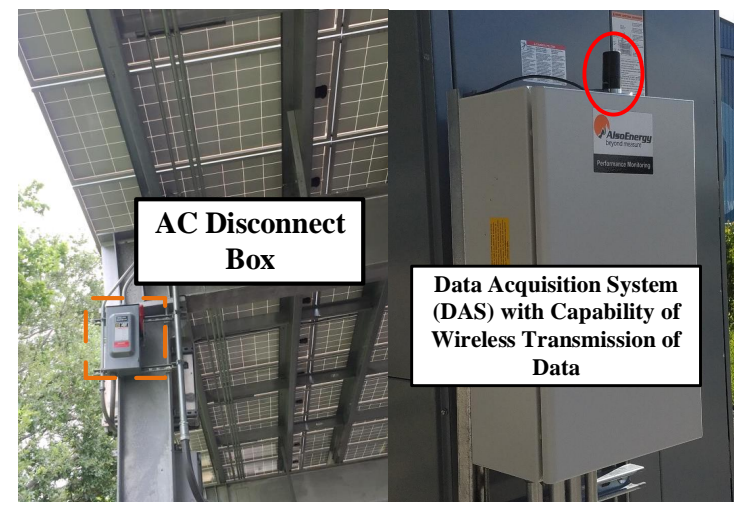

(e) (f)

Figure 5.1: PV Plant Components: a) 4460 PV modules of three different types but each with rated power around $315 \mathrm{~W}$, b) Transformer, Energy meter, and Main dosconnect at the Point of Common Coupling(PCC), c) Smart field inverters, d) Revolution Wireless Power Quality Recorder, e) AC disconnect box which is connected to the AC output of the inverter, f) DAS that measures the multivariate time-series data from inverters, meter and weather station and securely stores in a cloud server.

sensitive electronic devices and sophisticated data management devices, the assessment of power quality has become an inevitable task.

Many researchers have done power quality monitoring and assessment. In [201] the effect of photovoltaic power generation on current harmonics and voltage variations were investigated. This chapter found a correlation between the generated power and harmonic indices. A thorough power quality analysis for three different PV power plants with 4 
Table 5.1: Low-voltage System Classification and Distortion Limits [3]

\begin{tabular}{|lccc|}
\hline & $\begin{array}{c}\text { Special } \\
\text { Applications }\end{array}$ & $\begin{array}{c}\text { General } \\
\text { System }\end{array}$ & $\begin{array}{c}\text { Dedicated } \\
\text { System }^{2}\end{array}$ \\
\hline Notch Depth & $10 \%$ & $20 \%$ & $50 \%$ \\
THD (voltage) & $\mathbf{3 \%}$ & $\mathbf{5 \%}$ & $\mathbf{1 0 \%}$ \\
\hline
\end{tabular}

1- Special Applications include hospitals and airports.

2- A dedicated system is exclusively dedicated to the converter load.

Table 5.2: Maximum Current Distortion in Percent of Current [3]

\begin{tabular}{|cc|}
\hline Individual harmonic order $\mathrm{h}$ (odd harmonics) & Percent $(\%)$ \\
\hline $\mathrm{h}<11$ & 4.0 \\
$11<\mathrm{h}<17$ & 2.0 \\
$17<\mathrm{h}<23$ & 1.5 \\
$23<\mathrm{h}<35$ & 0.6 \\
$35<\mathrm{h}$ & 0.3 \\
Total demand distortion (TDD) (up to 50 harmonic) & $\mathbf{5 . 0}$ \\
\hline
\end{tabular}

MW, $1 \mathrm{MW}$, and 5MW capacities has been carried out by [202]. In this study 3 years of supply interruption and voltage dip data has been collected for three years and the observed disturbances compared to Grid-Code requirements. Monitoring of power quality impacts of a PV power generator has been conducted by [203]. Trends, waveforms and statistical analysis of the measurements was provided by this research. The power quality of transient variation in high solar and load conditions was presented in [204].

\subsection{Solution Approach}

PV plant description and case study scope is this chapter presents monitoring and assessment of a $1.1 \mathrm{MW}$ photovoltaic power plant at Florida International University, Miami, Florida. The assessment criteria were the IEEE 519 and 1547. Voltage and current distortion classifications are shown in Table 5.1 and 5.2. Revolution power quality recorder and AlsoEnergy power track software captured the harmonics and voltage data. The rest of this chapter is organized as follows. First, PV plant components are described and case 
study scope is presented. Then, real results from the PV power plant are illustrated and analyzed. The chapter is concluded in the last section.

\subsection{Test-bed Description and Case Study}

In order to analyze the voltage profile and power quality due to grid-tied PV, a comprehensive study was conducted on a 1.1MW PV power plant, shown in Fig. 6.1 (a), tied to a distribution feeder network in Miami, Florida. Three types of solar panels are installed in this PV power plant. Fig. 6.1 represents the Point of Common Coupling (PCC), where the PV system is connected to the distribution feeder.

Although the substation services eight feeders, this chapter deals with the impact of the plant on the feeder that the PV plant is connected to. The plant comprises: a) 4,460 PV modules of three different types, each with rated power around $315 W$; b) 46 smart string inverters, each of $24 k W$ size, and related combiner boxes shown in Fig. 6.1 (d); c) a local weather station that records irradiance and both ambient as well as module temperature; d) one cluster controller for monitoring the string inverters in real-time; e) an Elkor production meter to record the plant's net energy production (Fig. 6.1 (b)); f) Panel boxes for testing and disconnection, which have different breakers for each inverter module along that column; g) a SCADA controller at the plant-level that interacts with the cluster controller; h) Revolution $(\mathbb{R}$ Wireless Power Quality Recorder connected to the lower side of transformer at PCC and collects power quality data with a resolution of 1 minute from the plant including current and voltage THDs (Fig. 6.1 (d)); i) AC disconnect box connected to the AC output of the inverter and has necessary protection devices like switch and fuse (Fig. 6.1 (e)) ; and j) A Data Acquisition System (DAS) that measures the multivariate time-series data from inverters, meter and weather station and securely 
stores values in a cloud server (Fig. 6.1 (f)). The plant is currently at a $15 \%$ penetration into the grid, with a peak generation of $1.1 \mathrm{MW}$ on a clear, bright and sunny day.

Effective voltage profile analysis and power quality study are multi-step approaches which require reviewing the feeder monitoring criteria recommended by grid code requirements set by IEEE standard 1547.

\subsubsection{Placement of Revolution}

The most critical question to define compliance with IEEE 519 is, "Where is the best point to take the most precise measurements?" As said by the standard, the point of common coupling (PCC) is the best place to take all the measurements, since this is the nearest utility side point of the customer's service where a new utility is or could be supplied, usually at a substation or transformer. If the power quality meter is going to be installed at a transformer, the PCC can be on either the secondary or the primary side. However, as the secondary is close to the inverters and renewable power generators that are main sources of distortion, it is suggested that all measurements be read from the secondary to confirm compliance.

\subsection{Results and Analysis}

Based on the review of grid code requirements different feeder monitoring criteria were elicited. It is noteworthy that the concerned feeder, which serves 700 residential and 200 commercial customers, has three capacitor banks, one voltage regulator, and two transformers. Following subsections look at harmonics and voltage profile analysis. 


\subsubsection{Power Quality Study}

The power quality study under grid-integrated PV scenarios is examined in this section under two analyses: a) Power Ramp Rate study, and b) Harmonic Distortion study. While the power ramp rate study includes the analysis of high power density and high energy density ramp rates, the harmonic distortion study looks at both current as well as voltage THDs.

\subsubsection{Power Ramp Rate Study}

Ramp rates in power can be caused due to multiple reasons, of which cloud-induced power intermittencies are considered as a major characteristic. Single-sided power ramp rates, either ramp-up or ramp-down, at the point of interconnection (also called Point of Common Coupling or PCC), are represented in Fig. 5.2, which shows that majority of the power ramps are under $50 \mathrm{~kW} / \mathrm{min}$, and almost $20 \%$ of them exceed the $12 \%$ limit (corresponding to $140 \mathrm{~kW} / \mathrm{min}$ ). Thus, it is clear that ramp rates will pose serious threat as penetration levels increase. However, power ramps of this order can be easily mitigated and smoothed by integrating them with hybrid energy storage systems.

The power ramp rates have been categorized in this case study as high power and high energy density ramp rates, each of which are shown in Figs. 5.3 (a) and 5.3 (b), respectively. A high power density ramp rate with a $771 \mathrm{~kW} / \mathrm{min}$ of ramp down and a $799 \mathrm{~kW} /$ min ramp up was observed. Using supercapacitors minimizes impacts of these ramps. The high energy density ramp exhibits an energy deviation of $13.6 k W h$ in less than 5 minutes, effectively mitigated by battery banks. 


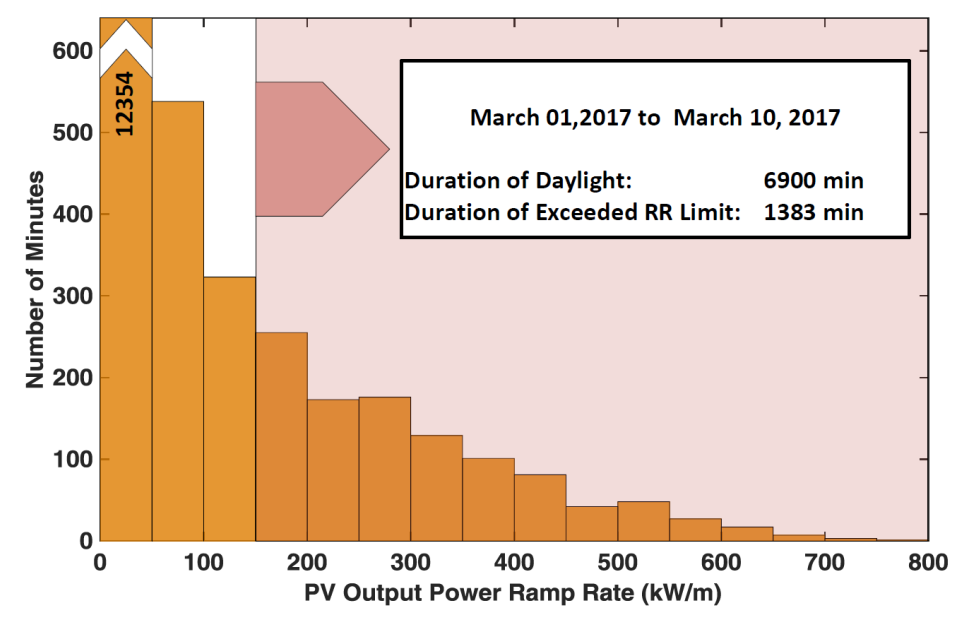

Figure 5.2: Histogram of real power ramp rates for Mar 1-10, 2017

\subsubsection{Recording Harmonics}

THD is not the best measure of PV-induced harmonics, since the fundamental current is reduced by PV generation, making harmonics larger by comparison and consequently increasing THD values. The top graph in Fig. 5.4 shows the current THD variations by Time and PV power production for four months in 2017. The lower graph is the side view of the top graph. It can be further noted that the current THD has an inverse relationship with the power output. Furthermore, there are no current THD violations when the PV generation exceeds $451 \mathrm{~kW}$ (41\% of nominal installed capacity). As it can be seen that the total current THD is highly sensitive to changes in irradiance but the total voltage THD does not maintain such a strong relation with the same (Fig. 5.5). During this period of four months in 2017 voltage THD was always within the IEEE standards limit. However, some violations, less than $0.3 \%$ noticed in March 2017. Nevertheless, these events happen at night time, when no PV generation is available, or in the morning, when the PV power output is still too low without any high fluctuations.

The effect of low solar irradiation level on injected current emissions is accounted for 


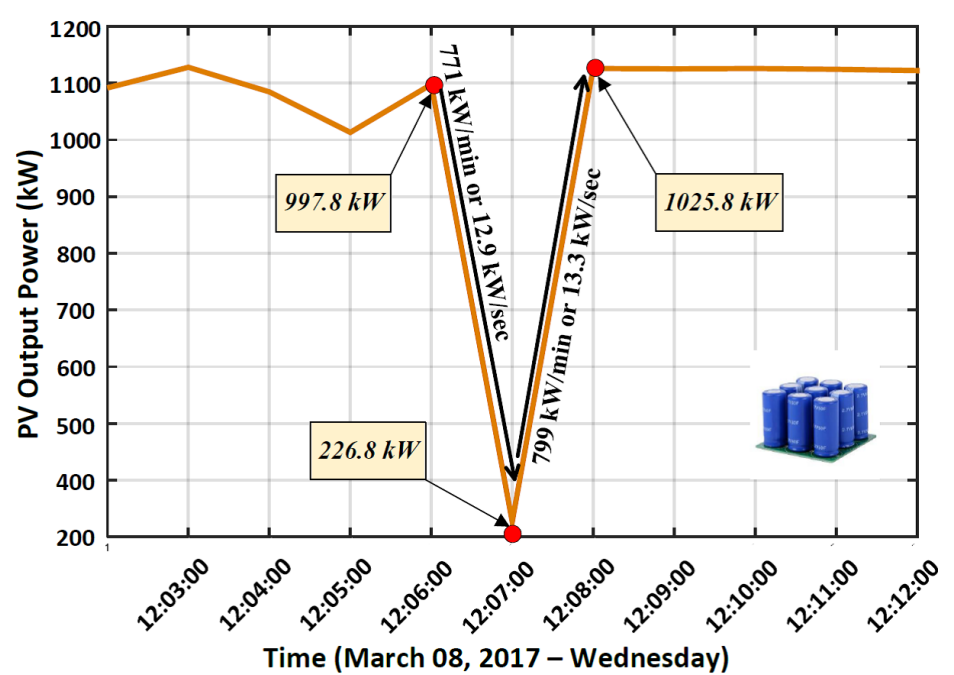

(a)

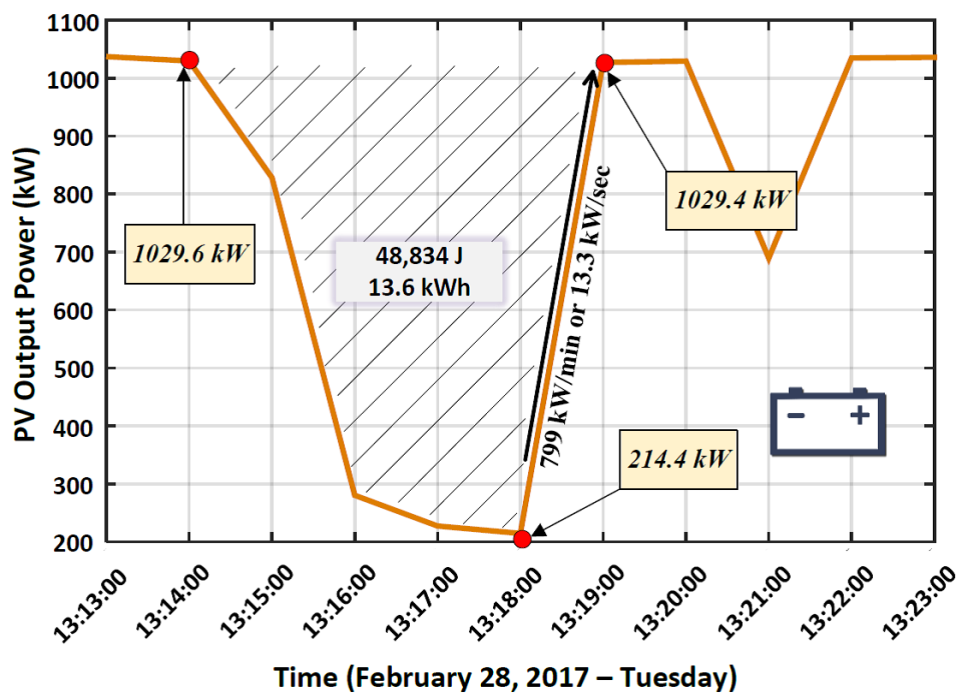

(b)

Figure 5.3: Power ramping scenarios for the PV power plant. a)High power density ramp rate profile, b) High energy density ramp rate profile. 

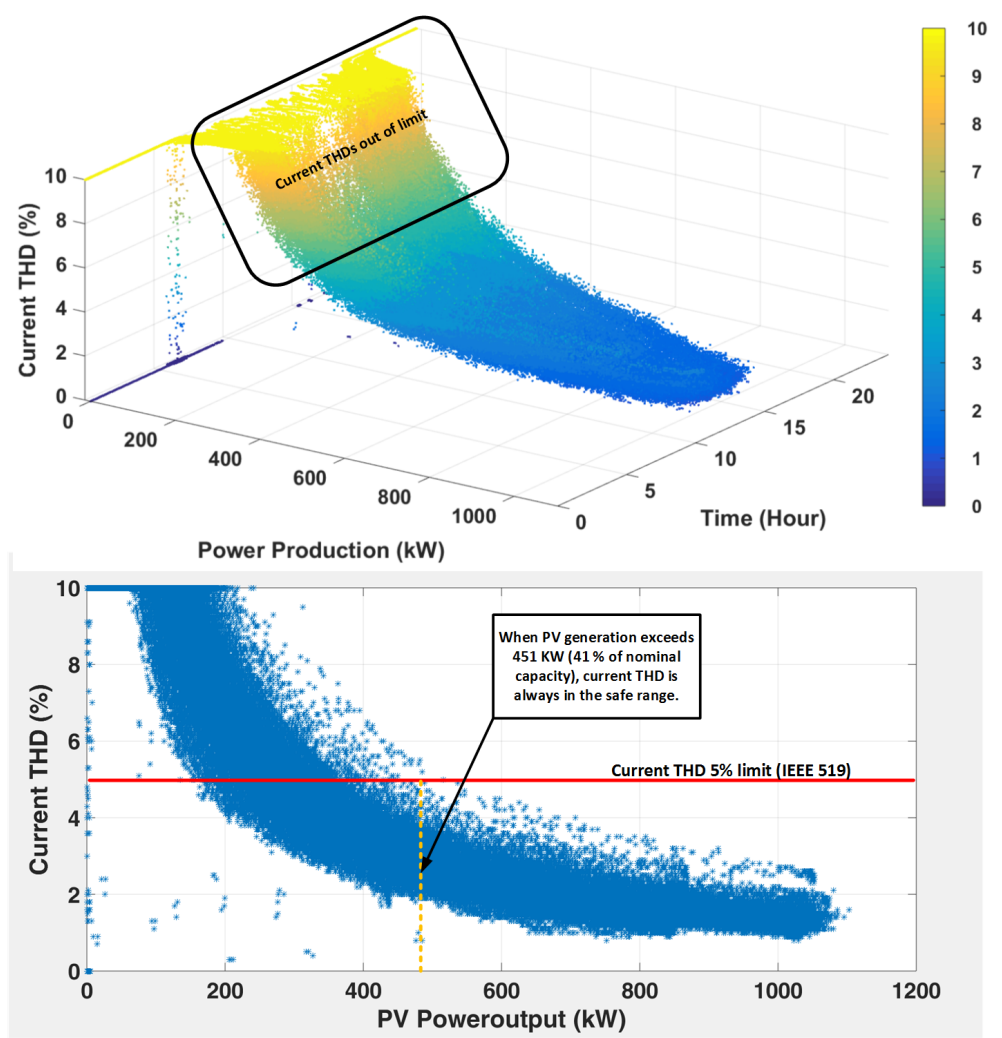

Figure 5.4: Current THD variation by Time and PV Power Production. (Data imported from Provision Power Quality meter).

in the case study. Monitored for a period of one month, the voltage and current THDs recorded for the power plant are plotted against the real power output in Figs. 5.6 (a) and 5.6 (b). It can be seen that the total current THD is highly sensitive to changes in irradiance but the total voltage THD does not maintain such a strong relation with the same. As the real power output crosses $870 \mathrm{~kW}$, the voltage THD does not exceed $4.1 \%$, with its values ranging between 4.5 and $5 \%$ when the power output is between 100 and $400 k W$.

The power output and current THD for March 11, 2017, when a lot of fluctuations in irradiance was observed, is depicted by Fig. 5.7. This goes on to validate the relation between power and current THD more precisely. It can be further noted that the shape of 


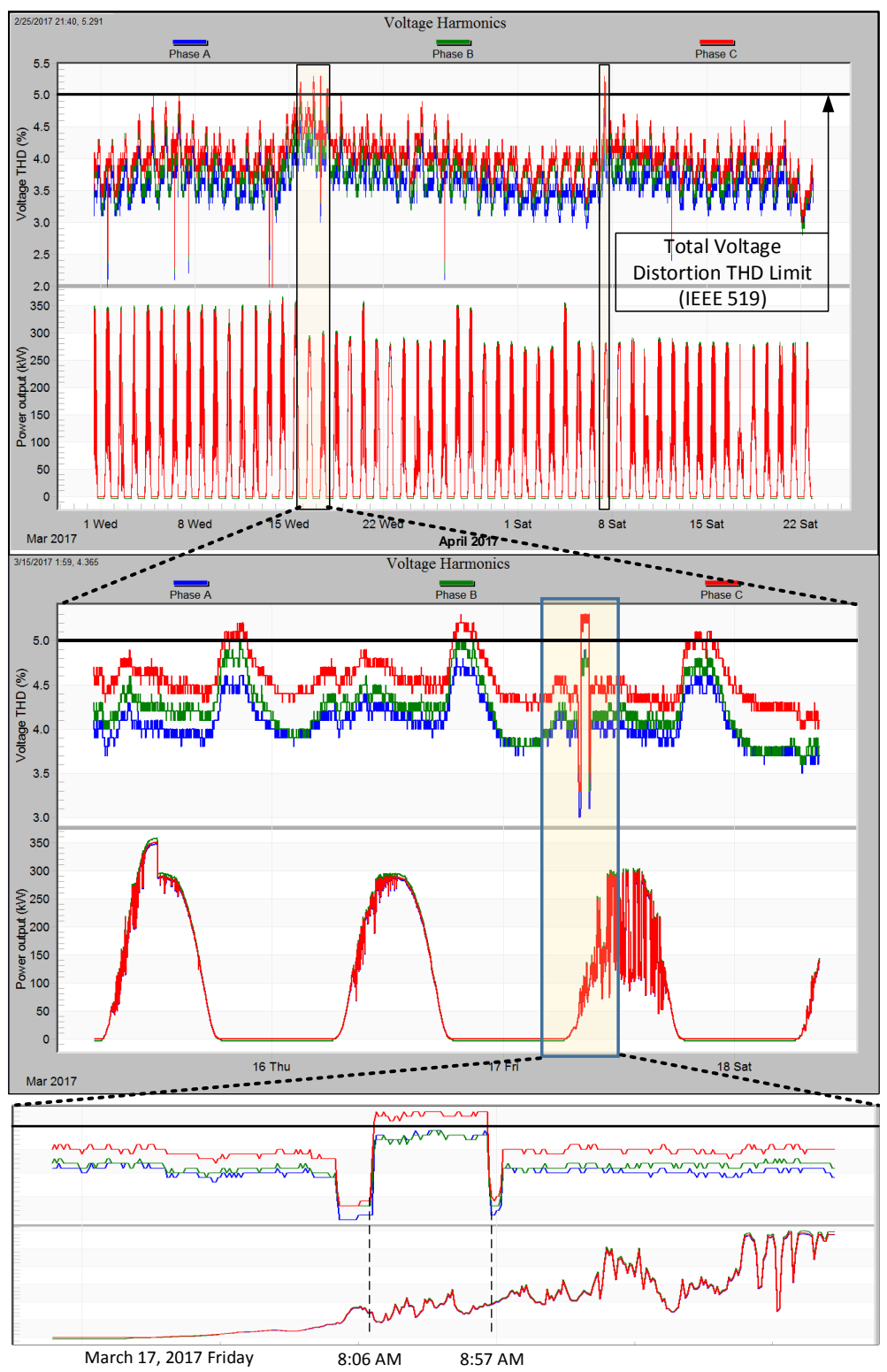

Figure 5.5: Voltage THD variation by Time and PV Power Production. (Data imported from Provision Power Quality meter).

current THD curve is nearly inverted to that of the output real power. 


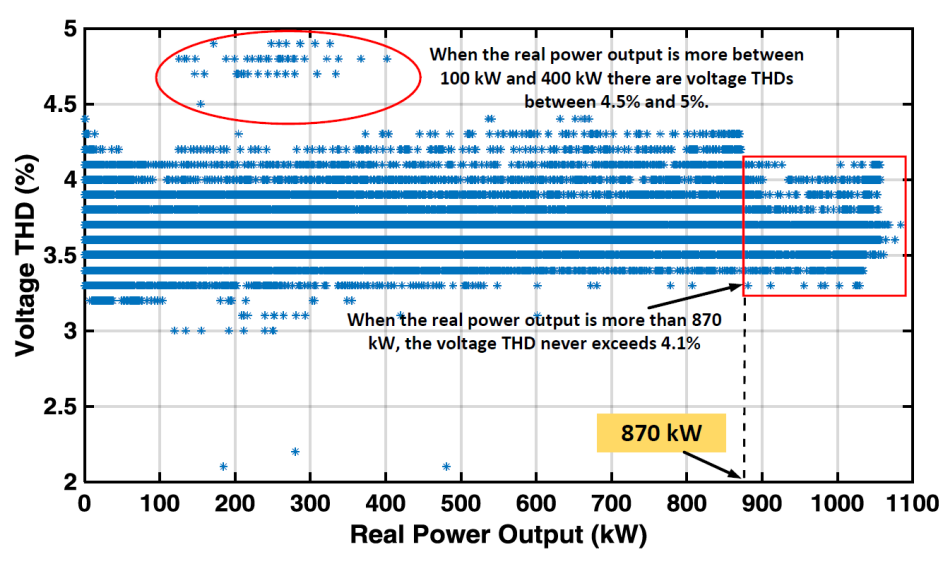

Real FIU Solar Canopy data from Feb 28, 2017 to Mar 28, 2017

(a)

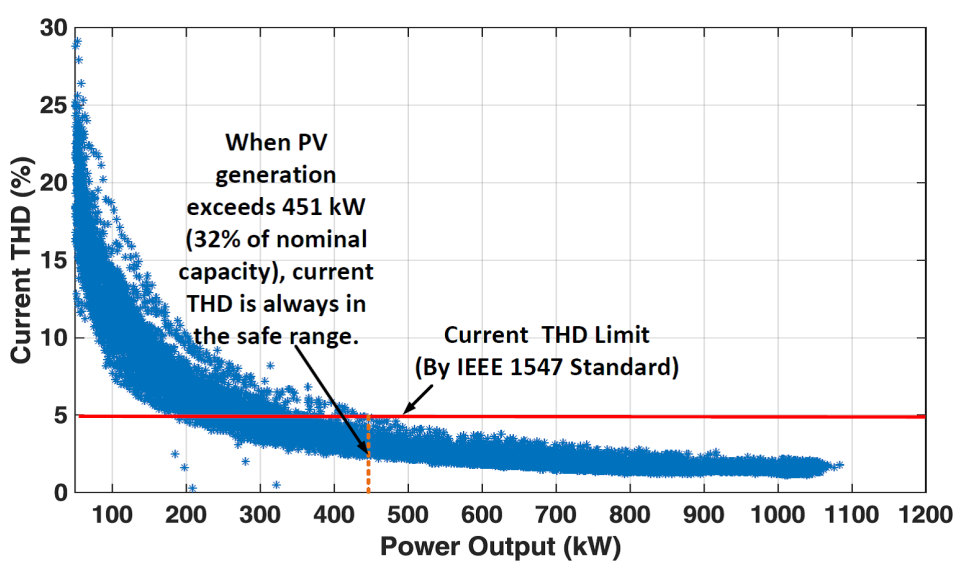

(b)

Figure 5.6: THDs monitored over 1 month for the case study. a) Voltage THD for the PV power plant, b)Current THD for the PV power plant. 


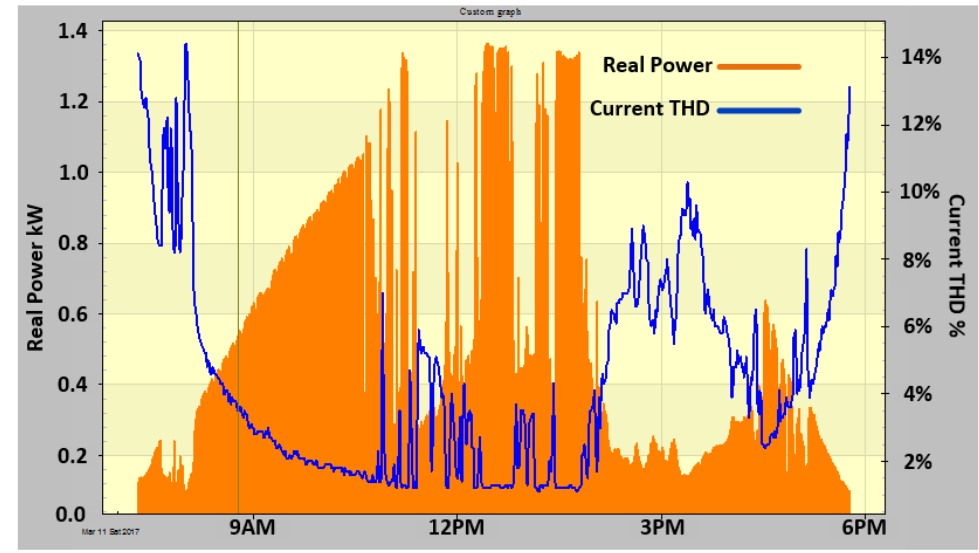

Figure 5.7: Current THD with respect to real power and time.

\subsubsection{Voltage Measurement}

The PV plant has been operational from July 19, 2016, and has since generated a cumulative energy of more than $1 G W h$.

The top graph in Fig. 5.8 shows the 60 cycle average of voltage variations by Time and PV power production for Feb 28, 2017 to July 27, 2017. The lower graph is the side view of the top graph. It can be seen that voltage does no exceed limits by when PV power output is beyond $800 \mathrm{~kW}$. This is opposite of what expected in high PV penetration. Therefore the voltage violations are not because of PV installation.

A time-series profile of the plant's current and voltage from July 31, 2016 to July 29, 2017 is shown in Fig. 5.10, from which a seasonal pattern can be estimated. Immediately after its commissioning, the plant experienced device failure issues due to which the data obtained was not of good quality (that is, it lacked consistency and accuracy). Hence, the initial few days of operation, from July 19 through 31, 2016, were removed from this analysis. It can be observed that the plant experiences a higher generation between the months of April and September, while it showcases a lower production profile during the winter months between November and January. The voltage profile analysis under 
grid-integrated PV scenarios is examined in this section. As shown in the left graph in Fig. 5.10, except from June 21, 2017 to July 13, 2017, the voltage profile was in the IEEE $1547 \pm 5 \%$ limits $(456 \mathrm{~V}-504 \mathrm{~V})$. By a closer look at selected days of that period $(07 / 01 / 2017-07 / 13 / 2017)$, it has been concluded that the voltage violations happened at the night time, therefore they are not due to PV generations.

The main cause of these fluctuations are the load profile of the feeder. When the working hour ends around 18:00 and employees start turning off the loads as computers, lights, air conditioners, etc. the voltages start increasing and reaches to the maximum of 510 volts around $4: 00$. Then around $8: 00$ in the morning, when the employees go to their work places, the load increases and voltage starts decreasing. In another study the ITIC (Information Technology Industry Council) and CBEMA (Computer Business Equipment Manufacturers Association) curve were generated to clearly represent Voltage events. These curves define different regions based on the sensitivity of computer equipment to the input voltage. As illustrated in Fig. 5.9, voltage events at the PCC may cause problems for sensitive electronic devices or computers, but not to the distribution feeder.

\subsubsection{Voltage Profile Analysis}

Analyzing real time voltage data from January 2017 to July 28 2017, does not show any voltage violation above 5\%, except for days May 17 to May 24, 2017 and June 21 to July 16, 2017. Real RMS voltage of the lower side of transformer, real PV power output data from Provision PQ meter is illustrated in Fig. 5.11. Since most of voltages out of limit were happened during this period, these days were selected. As it can be seen from the right side of Fig. 5.11, these violations happened mostly at night time, when there is no PV output. Moreover, the overall voltage rises throughout the day as the load decreases (during late night). Therefore, these voltage violations are not due to PV penetration to 

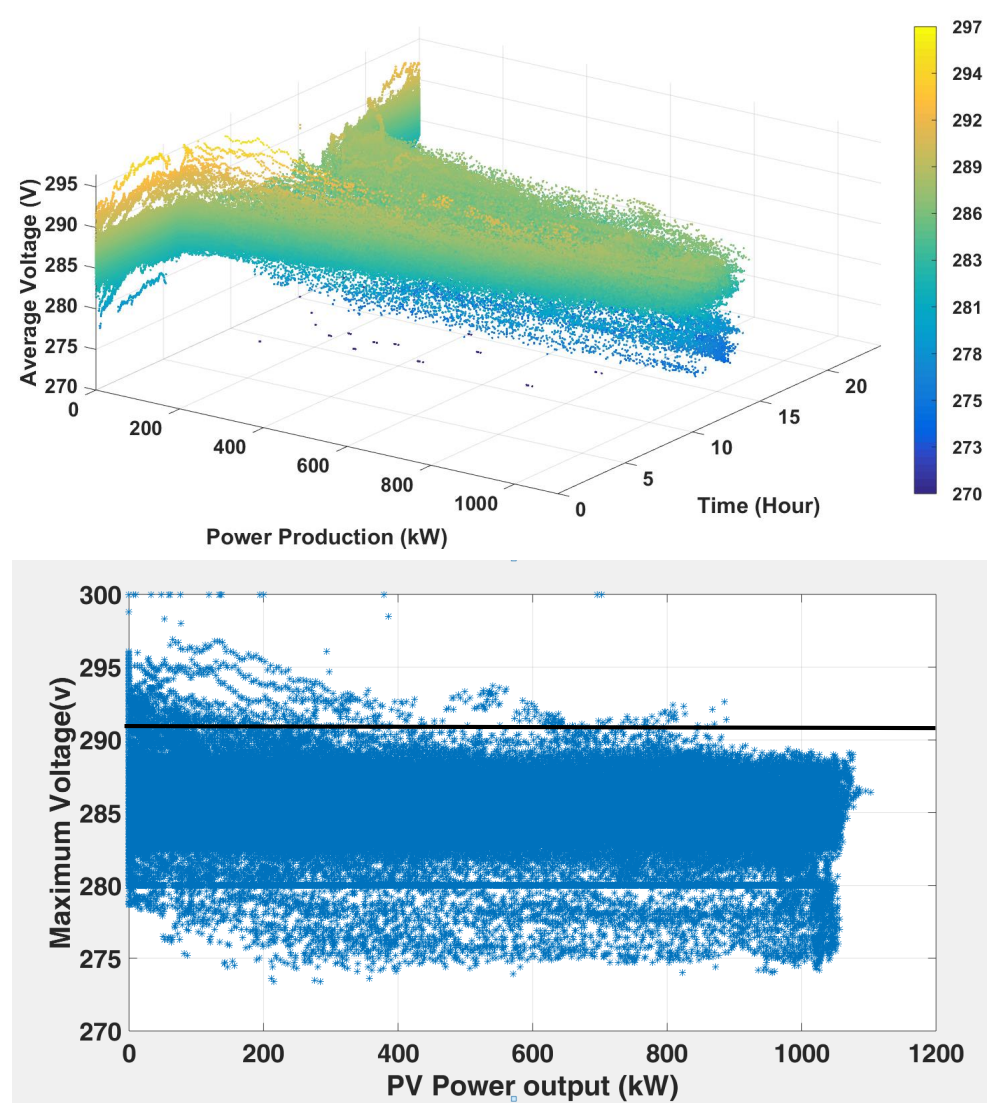

Figure 5.8: Voltage variation by Time and PV Power Production (Data imported from Provision Power Quality meter).

the feeder. The voltage profile analysis under grid-integrated PV scenarios is examined in this section as two analyses: a) Steady-state, and b) Time-series. While the steadystate analysis determines the worst-case feeder response that would occur when the PV generation changes drastically from zero to maximum, time-series analysis is conducted for the load or PV time-of-day coincidental scenarios.

\subsection{Steady-State Scenario Study}

Considering steady-state scenario is static in nature, the load flow analysis for the system is conducted for different use-cases. Each use-case has two scenarios that define it: the 


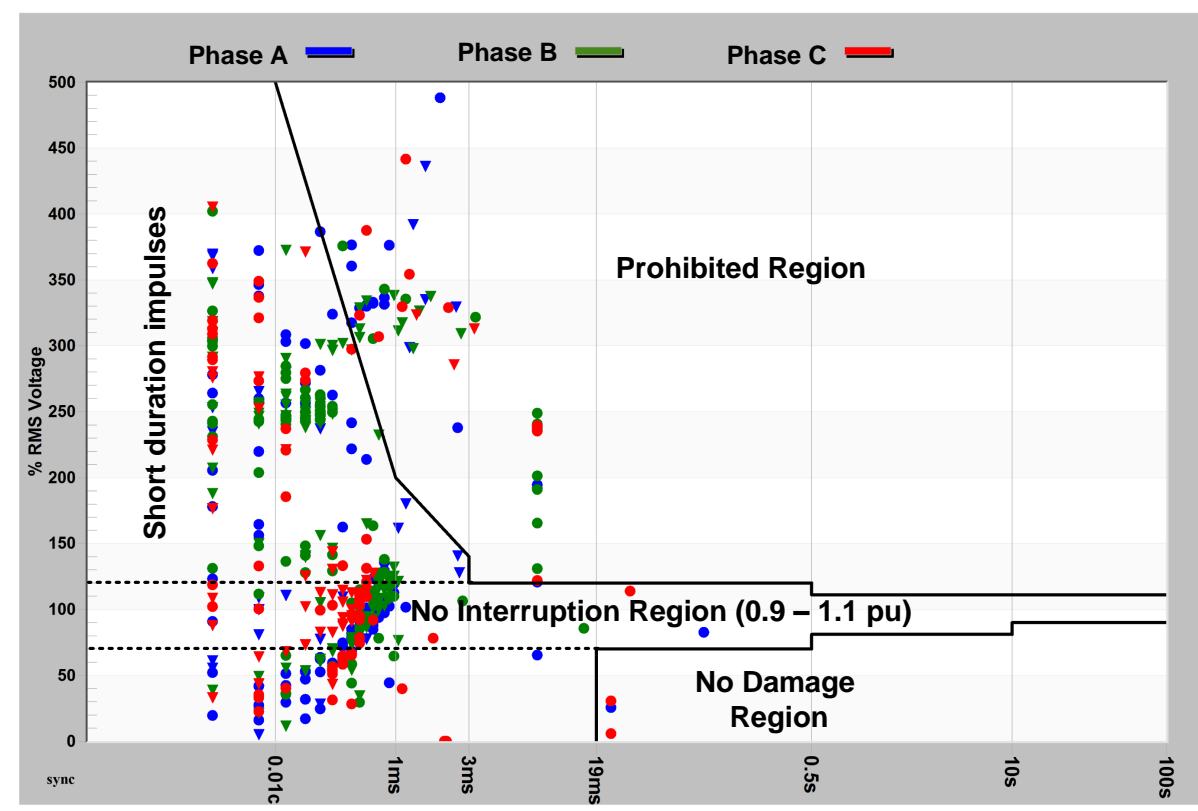

Figure 5.9: ITIC (CBEMA Curve) of Photovoltaic power plant at FIU.

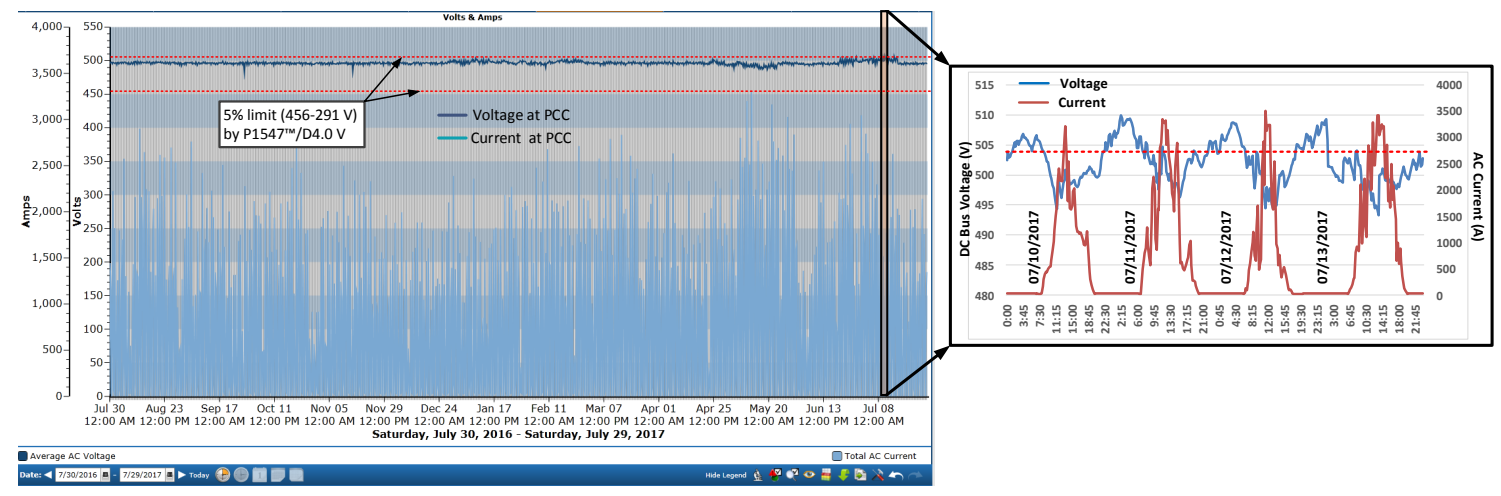

Figure 5.10: Photovoltaic Power Plant Voltage and Current at the PCC.

dynamic load profile scenario, and the different levels of PV penetration scenario. Although the existing level of penetration is $15 \%$, this study considers futuristic scenarios where the penetration could go as high as $140 \%$ of the peak load. The load scenarios, on the other hand, could be the Peak Daytime Load (PDL) day, or the Minimum Daytime Load (MDL) day. Accordingly, the mapping between these two classes of scenarios as shown in Fig. 5.12 would create multiple use-cases for which the results are shown. 

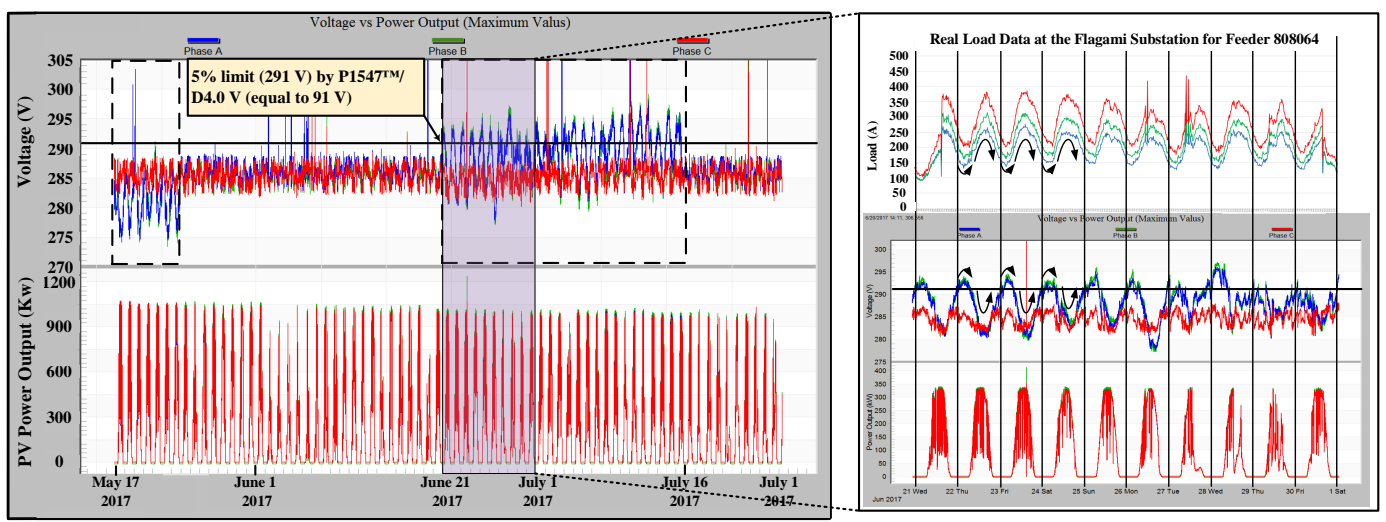

Figure 5.11: Maximum RMS values for voltage, Power output, and Load

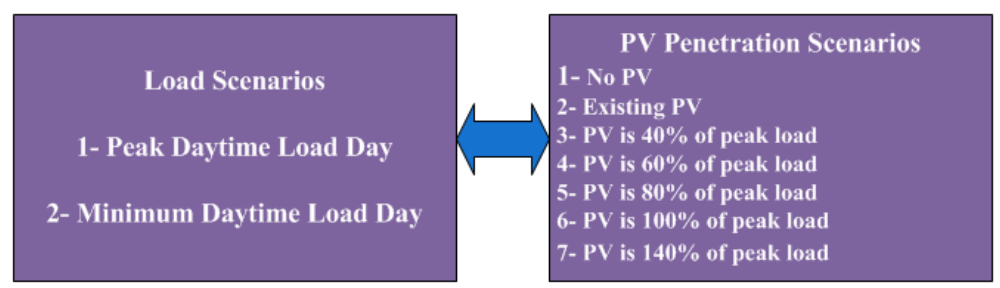

Figure 5.12: Mapping scenario classes for use-case generation

\subsubsection{Voltage Deviation Study:}

In order to conduct this study, the two load scenarios were each mapped to two different penetration levels (existing and 60\%), to generate four use-cases. It is to be noted that for this setup, the voltage deviation should not exceed 0.3V. Fig. 5.13 (a) shows the voltage deviation for the PDL scenario under existing penetration level with respect to the distance from the substation. It is a general trend that the deviations subside as the distance increases. Moreover, it is seen that the values are well below the recommended one. However, at $60 \%$ penetration, shown in Fig. 5.13 (b), although the deviations are still below the threshold, they are dangerously close to exceeding it, especially at distances closer to the substation. Much similarly, Fig. 5.14 shows the deviation in voltage for the MDL scenario again for existing and $60 \%$ penetration levels with respect to distance 


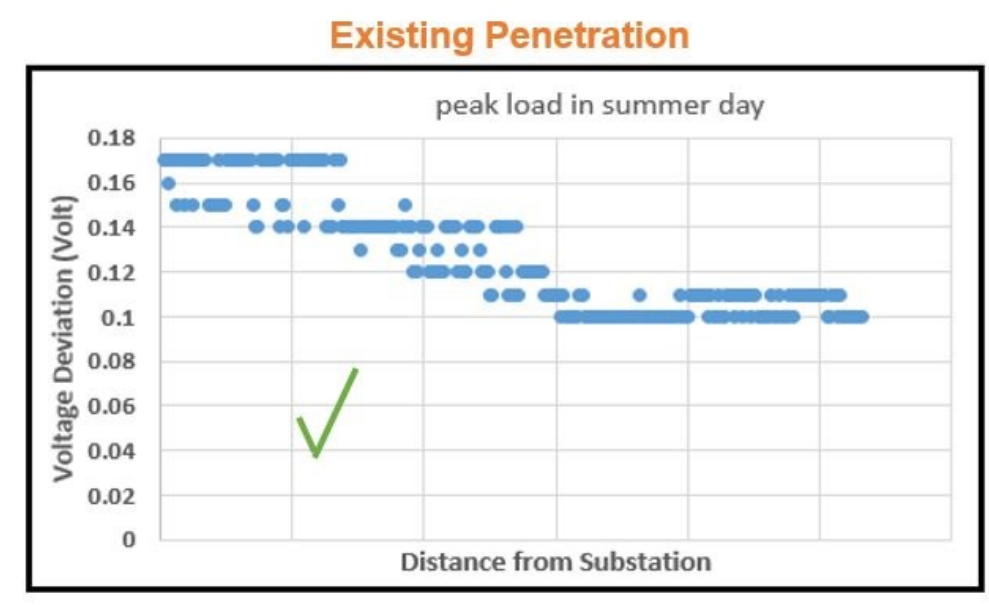

(a)

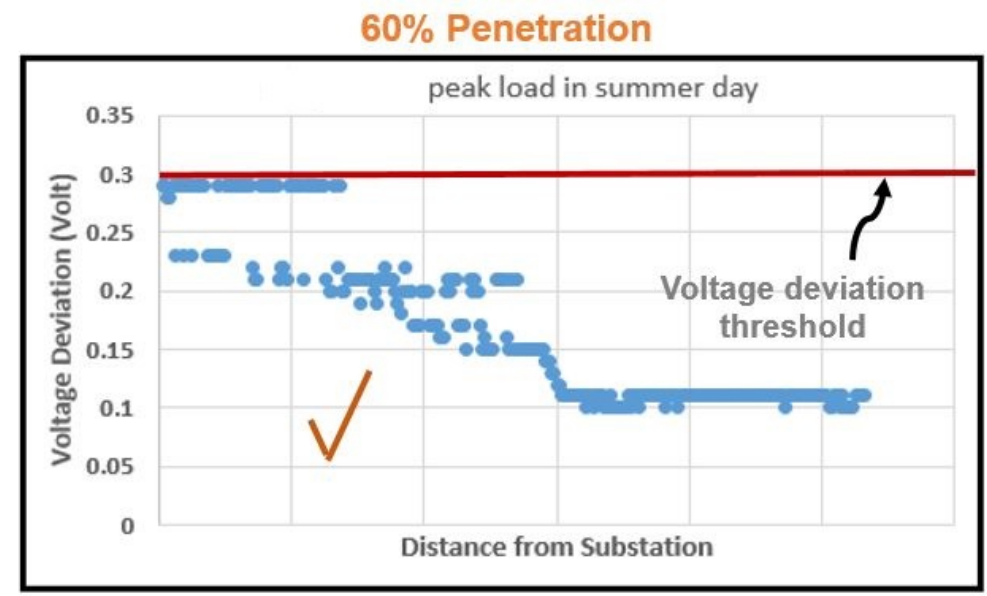

Figure 5.13: Voltage deviation study for PDL scenario. a)Existing Penetration, b) $60 \%$ Penetration

from the substation. While the deviations are below the stipulated threshold for existing penetration level, they exceed the same under levels of $60 \%$ penetration significantly by $0.15 \mathrm{~V}$, at a distance closest to the substation. Considering PV is located at the end of the feeder, no significant impacts were observed. 


\section{Existing Penetration}

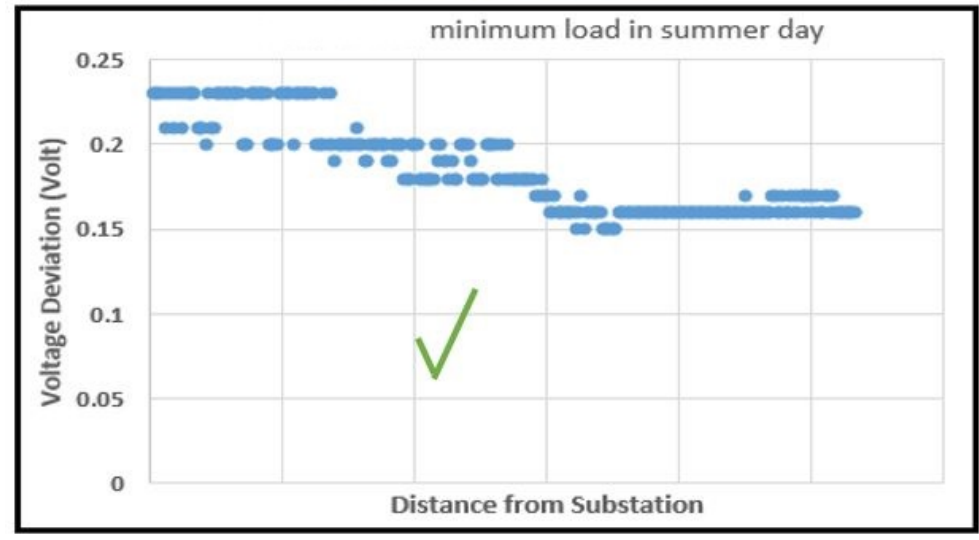

(a)

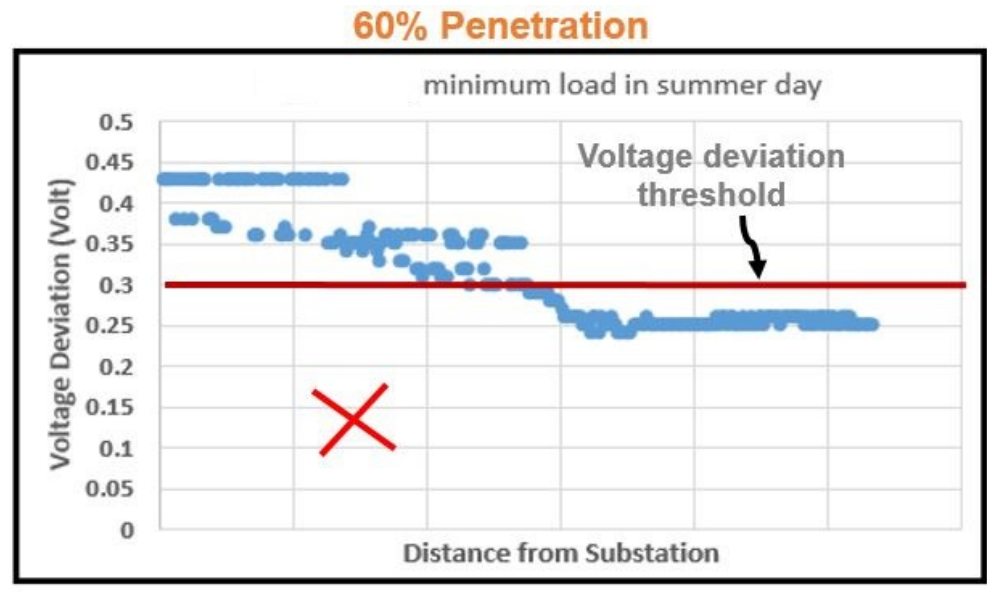

(b)

Figure 5.14: Voltage deviation study for MDL scenario. a) Existing Penetration, b) $60 \%$ Penetration. 


\subsubsection{Feeder Loss Study:}

The feeder losses $(\mathrm{kW})$ for the PDL and MDL scenarios under different penetration levels is illustrated graphically in Fig. 5.15. It can be observed that the losses show a gradually declining trend for the PDL scenario where the losses drop by a factor of $66 \%$ from 0 to $140 \%$ penetration, but the same cannot be said for the MDL scenario, where the losses initially seem to decline, with the lowest losses observed for $60-80 \%$ penetration, but steadily climb up again as the penetration hikes to $140 \%$. It is incidentally seen the losses are more or less the same for both extremities of the penetration level in this load scenario.

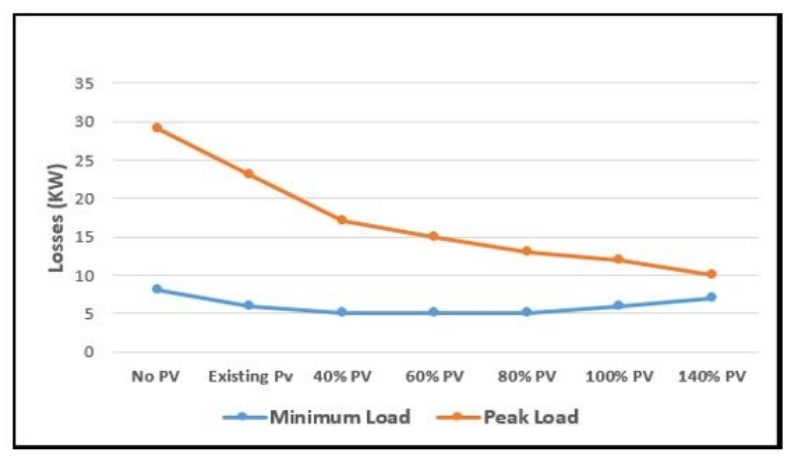

Figure 5.15: Feeder Losses for MDL and PDL Scenarios

\subsubsection{Time-Series Scenario Study}

Unlike the steady-state scenario, the time-series study requires a different kind of system modeling. All distributed loads are assumed to be modeled as urban residential loads and all spot loads are modeled as urban commercial loads in order to construct the load model for the study, shown in Fig. 5.16. Further, two scenarios are considered here: a sunny day where the sky is clear and bright blue, and a cloudy day where the sky is overcast. The irradiance models for both these scenarios using 15-min irradiance interval data are shown in Fig. 5.16. However, the one-hour resolution data is used by taking an average from 


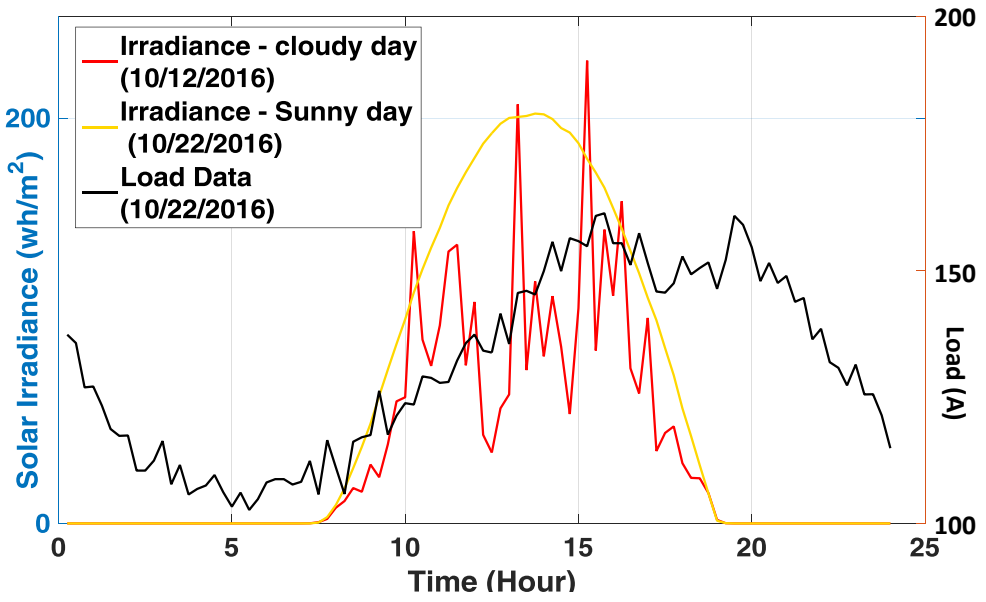

Figure 5.16: Irradiance and Load models for System Modeling. Irradiance model for a sunny day in October 2016, Irradiance model for cloudy day in October 2016, Load Model

15-min resolution data. Further, three different PV inverter control modes are considered for this study, namely: Power Factor control, Volt/VAr control, and Volt/Watt control.

For $\mathrm{PF}$ control mode, $\mathrm{PF}=0.85$ was considered. The voltage profile analysis was then conducted for a sunny day scenario, as depicted by Fig. 5.17, by varying PV penetration levels as: existing (sky blue), 40\% (orange), 60\% (grey), 80\% (yellow), 100\% (deep blue), $120 \%$ (green), and 140\% (dark blue). As shown in Fig. 5.17 (a), the maximum voltage exceeds the threshold at $60 \%$ penetration and above for $\mathrm{PF}=0.85$. Similarly, the threshold is exceeded at the same level of penetration even for Volt/VAr control mode, shown in Fig. 5.17 (b). This might prompt the inverters to be operated at $P F=1$ considering the limit is not violated. However, this contradicts the recommendations made by IEEE 1547 which requires inverters to operate in Volt/VAr control mode. Hence, appropriate mitigation strategies are required. It can be further noted that the number of switching operation is 6 for $\mathrm{PF}=0.85$, and jumps to 36 for Volt/VAr mode

The above study was now repeated for a cloudy day scenario, and the number of switching operations was observed for $\mathrm{PF}=0.85$, and Volt/VAr control modes. The maxi- 


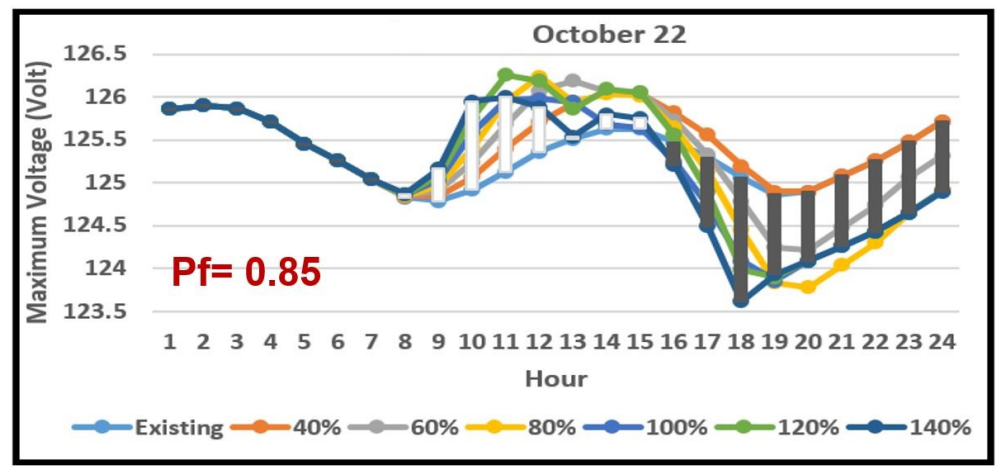

(a)

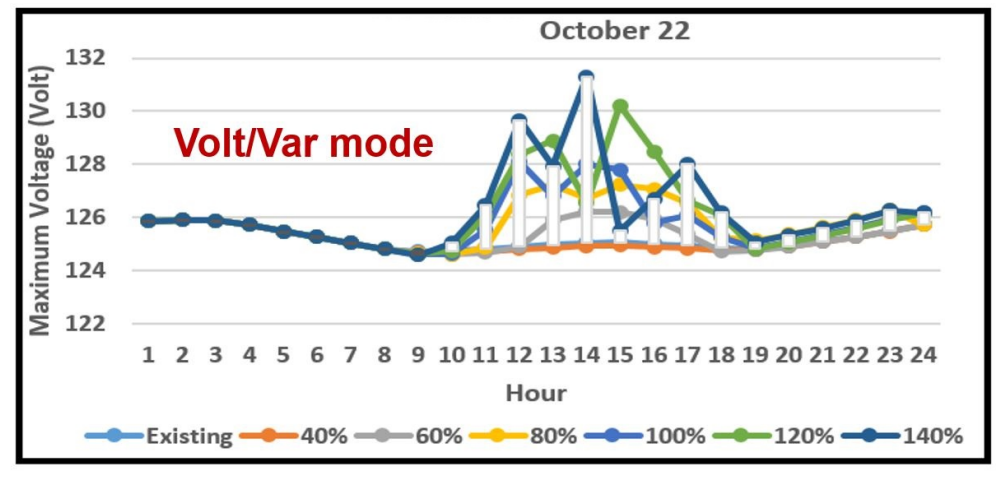

(b)

Figure 5.17: Maximum Feeder Voltage for different control modes on sunny day a)Power Factor $=0.85 \mathrm{~b}$ ) Volt/VAr mode 


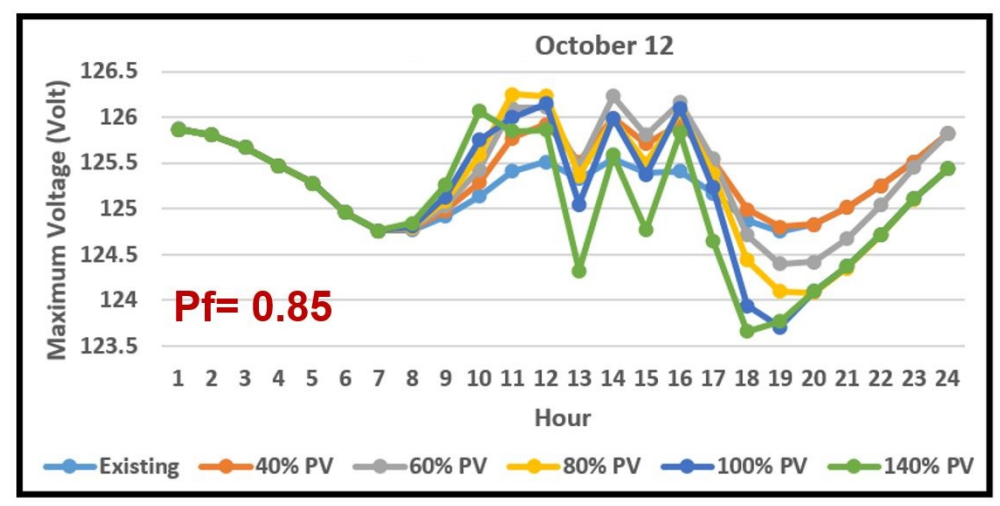

(a)

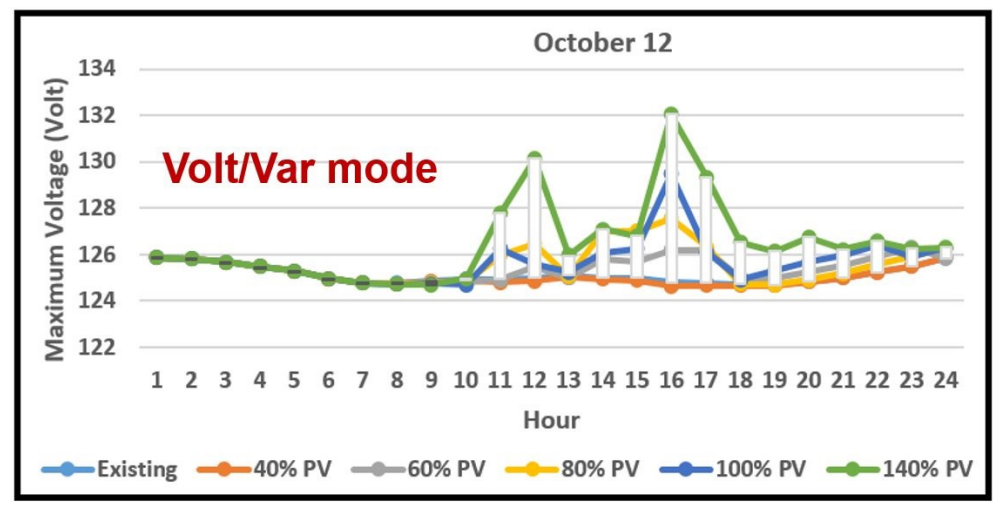

(b)

Figure 5.18: Maximum Feeder Voltage for different control modes on cloudy day a) Power Factor $=0.85$ b) Volt/VAr mode

mum feeder voltage variations are shown in Fig. 5.18. At $60 \%$ penetration level and beyond, the maximum feeder voltage exceeds the threshold for both $\mathrm{PF}=0.85$ and $\mathrm{Volt} / \mathrm{VAr}$ modes, depicted respectively in Figs.5.18 (a) and 5.18 (b), with corresponding switching operations as 6 and 69 . When compared with their operations on a sunny day, it can be observed that when PV inverters operate in Volt/VAr mode on a cloudy day, the voltage regulators undergo switching operations nearly twice more, which significantly reduces their performance and spells adverse effects on the grid.

A consolidated representation of the number of switching operations for the 8 voltage regulators operating under various control modes in both sunny as well as cloudy days. As can be seen in Table 5.3, the number of switching operations is relatively stable be- 
Table 5.3: Total Numbers of Switching Operations.

\begin{tabular}{|c|c|c|c|c|c|c|c|c|}
\hline & \multicolumn{2}{|c|}{ PF= 1 } & \multicolumn{2}{c|}{ PF= 0.85 } & \multicolumn{2}{c|}{ Volt/Watt } & \multicolumn{2}{c|}{ Volt/Watt } \\
\cline { 2 - 9 } & Sunny & Cloudy & Sunny & Cloudy & Sunny & Cloudy & Sunny & Cloudy \\
\hline $\mathbf{2 0 \% ~ P V ~}$ & 1 & 2 & 1 & 2 & 1 & 2 & 1 & 2 \\
\hline $\mathbf{4 0 \%}$ PV & 1 & 2 & 1 & 2 & 1 & 2 & 1 & 2 \\
\hline $\mathbf{6 0 \%}$ PV & 1 & 2 & 2 & 3 & 2 & 3 & 5 & 16 \\
\hline $\mathbf{8 0 \%}$ PV & 1 & 2 & 3 & 3 & 6 & 6 & 22 & 42 \\
\hline $\mathbf{1 0 0 \%}$ PV & 2 & 2 & 6 & 6 & 8 & 11 & 36 & 69 \\
\hline $\mathbf{1 4 0 \%}$ PV & 2 & 3 & 11 & 15 & 16 & 19 & 44 & 113 \\
\hline
\end{tabular}

tween 1 and 2 until 100\%, with the number creeping to 3 on a cloudy day scenario. When $\mathrm{PF}=0.85$, the number of operations show a steady rise with penetration levels, peaking at 11 and 15 operations for sunny and cloudy days, respectively at $140 \%$ penetration. Similarly, when operated under Volt/Watt mode, the operations peak at 16 and 19 for the same penetration level, represented in Table 5.3. Finally, under Volt/VAR, maximum number of switching operations is observed, with 44 on sunny and 113 on cloudy day for maximum penetration scenario considered in this chapter. This supports the hypothesis that number of operations increases with penetration levels, and that Volt/Watt and Volt/VAR modes are more dramatic than PF. This might prompt the inverters to be operated at PF considering the limit is not violated. However, it contradicts recommendations made by IEEE 1547 which require inverters to operate in Volt/VAr mode. Hence, mitigation strategies are required.

\subsection{Summary}

This chapter presents power quality monitoring results of an 1.1 MW Photovoltaic power plant installed at Florida International University. Site description and list of components are provided. Base on the analysis, steady state voltage violations has been occurred on Jun 20 to Jul 16 (Over-voltages). However, some spikes happen during the period of study, which were not because of PV installation. Based on ITIC curves momentary 
voltage spikes can cause problems for sensitive electric devices and computers, but not to the grid.Voltage THD Violation only happens on phase and they were below $5.3 \%$. Voltage THDs may not cause major problems in the Feeder. However, current THD has an inverse relationship with PV power production. When PV production is lower than $451 \mathrm{~kW}$, current THDs exceed 5\% limit the IEEE 1547.

A system model and data from the plant's data acquisition unit and power quality recorder was constructed. Multiple use-cases and scenarios were delineated for the two studies. Power quality issues were studied using high resolution data for current and voltage THDs based on real measurements. It was concluded that no problematic issues persisted at the existing penetration level of 1.1 MW. Current THDs over 5\% has been increased when the power output is less than $451 \mathrm{~kW}$ and it has a tight connection to the output power. Voltage profile analyses for steady-state and time-series scenarios revealed that at $60 \%$ penetration level, significant impacts due to voltage deviation and feeder losses could be observed. Further, the number of switching operations for voltage regulators increases dramatically when PV inverters operate in Volt/VAr control mode, followed by Volt/Watt, and finally Power Factor. Although unity power factor causes least number of operations, the grid codes require the use of Volt/VAr mode for inverter control. Hence, strategies to mitigate these impacts are required. 


\section{CHAPTER 6}

\section{A NEW TOPOLOGY OF HIGHER ORDER POWER FILTER FOR SINGLE-PHASE GRID-TIED VOLTAGE SOURCE INVERTERS}

\subsection{Overview}

In order to reduce the influence of the grid harmonic currents and voltages, harmonic compensation is regularly implemented for a grid-tied inverter. In this study a new topology of a higher order power filter for single-phase grid-tied voltage-source inverters (VSIs), named $L(L C L)_{2}$, is presented. The subscript is added to the name to prevent confusion with the $L L C L$ filter. In the proposed design, the inverter side inductance is divided into three parts, and the grid side inductor is removed. Also, an additional resonant branch at the double of the switching frequency is added to the traditional $L L C L$ filter to attenuate high-frequency harmonics. The overall inductance of the recommended filter is smaller than the $L L C L$ filter. A comparative study and discussions on the subject of the traditional $L L C L$ filter and the proposed $L(L C L)_{2}$ filter have been conducted and assessed through an experimental hardware implementation on a $700 \mathrm{~W}, 120 \mathrm{~V} / 60 \mathrm{~Hz}$ single-phase gridtied inverter. Furthermore, a straightforward engineering design benchmark is suggested to discover parameters of the $L(L C L)_{2}$ filter. Moreover, stability analysis, loss analysis and an optimization of the $L(L C L)_{2}$ filter parameters have been conducted in this study. The analysis shows that in comparison with the $L L C L$ filter, the $L(L C L)_{2}$ filter not only has lower voltage drop and less total inductor size, but also has improved performance in decreasing high order current harmonics. 


\subsection{Problem Statement}

As renewable energy systems, especially photovoltaics, are gaining more and more consideration, the grid-tied inverter has been extensively accepted. In order to limit unnecessary current harmonics, which are typically created by pulse width modulation (PWM), a low-pass power filter is regularly placed between a voltage-source inverter (VSI) and the grid $[11,205]$. L-filters are normally used, but they have the drawbacks of slow dynamic response and big inductor value $[14,206]$. In comparison to a first-order $L$ filter, an $L C L$ filter can satisfy the standards for the grid interconnection with notably smaller size and cost, primarily for applications in several kilowatts [207]. Due to the growing cost of copper, various methods have been implemented to cut down the price of the power filter. A practical procedure is to increase the switching frequency of the inverter where the method, surely, depends on the device proficiency and cost.

Other measures, such as special topologies or controls, have been focused on by researchers [208]. With the intention of achieving a higher switching frequency and efficiency, a three-level neutral point clamped (NPC) converter as a high-power renewable energy grid interface was presented in [209]. In [210] and [211], dual-mode timesharing control methods for single and three-phase inverters, correspondingly, were recommended to enlarge the modulation index and decrease the power filter size. However, these suggestions make the control methods or topologies more complicated, which leads to less reliability. Besides, it is very problematic for a dual-mode timesharing type inverter to reduce the harmonics or produce reactive power for the power grid. Ref. [212] presents a new design technique, which employs SiC-type power devices for switching frequency optimization and improvement of the structure of the $L C L$-type output filter in transformerless PV inverters.

The total power loss of the $L C L$ filter was used as the optimization factor in the de- 
signing of the filter parameters in [213]. The work in [213], explores the $L C L$ filter design method from the perspective of efficiency and reduction in size and weight (and therefore cost). Nowadays, most power electronic designs are limited by thermal constraints. Power dissipation and surface area have a major influence on temperature change. The volume and size of the component are two measures that the surface area of the object is directly linked with. Moreover, when it comes to the industry, price is a vital aspect of selecting the power filter in a grid-tied inverter. Recently, for more reduction of the inductor size, the $L L C L$-filter has been proposed in [214]. Unlike the $L C L$ filter, it can save on total cost and material since the grid-side inductance can be significantly reduced.

In [215], the topology of multiple shunt $R L C$ trap filters has been analyzed, but the detailed design process was not given. Several optimized-filter designs, common-mode voltage suppression methods, recommendations, and standards were also proposed in [216-218].

\subsection{Solution Approach}

The objective of chapter 6 is to propose a modified high-order filter design, named $L(L C L)_{2}$ filter, based on the $L L C L$ filter. The proposed filter can reduce the harmonics at the switching frequency and multiples of the switching frequency while saving the total inductance and thereby resulting in size reduction of the filter. The most important role of the gridside inductor in the traditional $L L C L$ filter is to decrease the harmonics around twice of the switching frequency. In the new topology, this inductor is removed, and the inverterside inductor is split into three parts. Then, two resonant traps at the switching frequency and double of the switching frequency are inserted in between the inverter-side inductor. The proposed filter is able to attenuate the current ripple components better than the $L C L$ and the $L L C L$ filters. 


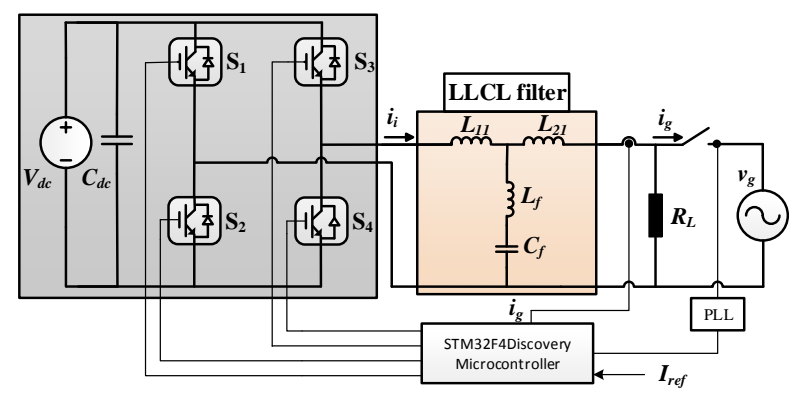

Figure 6.1: Schematic diagram of the $L L C L$ filter.

The outline of the rest of chapter 6 is as follows: First, the principle of an $L L C L$ filter is presented. Then, a new engineering design procedure and parameter optimization of the high-order power filter is proposed and analyzed. Additionally, the close-loop Stability is assessed. Finally, experimental results on a $700 \mathrm{~W}, 120 \mathrm{~V} / 60 \mathrm{~Hz}$ single-phase grid-tied inverter prototype with $L L C L$ and $L(L C L)_{2}$ filters are carried out and compared to confirm the correctness of theoretical analysis.

\subsection{Principles of The $L L C L$-Filters}

The circuit configuration of an $L L C L$-filter-based single-phase grid-tied VSI is illustrated in Fig. 6.1. The output voltage and current of the inverter are represented as $v_{i}$ and $i_{i}$, and the grid voltage and current are represented as $v_{g}$ and $i_{g}$. The switching frequency is shown as $f_{s}$ (in hertz) or $\omega_{s}$ (in radians per second). To simplify the analysis, the power grid is assumed to be a perfect voltage source with zero impedance, to supply a continuous voltage at the frequency of $60 \mathrm{~Hz}$.

The instantaneous output voltage $v_{i}(t)$ of the single-phase full-bridge VSI can be calculated as (6.1) while it is utilized under the situation of sine-triangle, unipolar, and asymmetrical regular sampled PWM [219]. 


$$
\begin{aligned}
& v_{i}(t)=m V_{d c} \cos \left(\omega_{0} t\right) \\
& +\sum_{k=1}^{\infty} \sum_{n= \pm 1}^{ \pm \infty} \frac{2 V_{d c} J_{n}(k \pi m)}{k \pi} \sin \left(\frac{n \pi}{2}\right) \cos \left(k \omega_{s} t+n \omega_{0} t\right)
\end{aligned}
$$

where $m$ is the modulation index, $V_{d c}$ is the dc-link voltage, $\omega_{0}$ is the fundamental frequency, and $J_{n}(x)$ is referred as the integrals of the Bessel function, which is known as: $J_{n}(x)=\int_{0}^{\pi} \cos (n \pi-x \sin t) d t$, representing the sideband harmonic magnitude.

The inverter output impedance while $\omega \neq \omega_{0}$ can be written as

$$
\begin{aligned}
& Z_{0}(j \omega)=\left.\frac{v_{i}(j \omega)}{i_{i}(j \omega)}\right|_{v_{g}(j \omega)=0} \\
& =\frac{\left(L_{11} L_{21} C_{f}+\left(L_{11}+L_{21}\right) L_{f} C_{f}\right)(j \omega)^{3}+\left(L_{11}+L_{21}\right)(j \omega)}{\left(L_{21}+L_{f}\right) C_{f}(j \omega)^{2}+1}
\end{aligned}
$$

The grid-side current $i_{g}$ is supposed as the ideal continuous current at the fundamental frequency (Considering the harmonic current recommendation in IEEE 519-2014 and IEEE 1547.2-2008 [220,221]). With that in mind, the branch circuit of inductor $L_{21}$ can be seen as being opened by considering the effects of the inverter high-frequency harmonics.

The amplitudes of harmonics of inverter-side current $i_{i}$ can be derived as

$$
\left|I_{H}\right|_{\omega \neq \omega_{0}}=\frac{\left|V_{H}(n, k)\right|}{\left|Z_{0}(j \omega)\right|}
$$

where the amplitude output voltage harmonic $V_{H}(n, k)$ is

$$
V_{H}(n, k)=\left|\frac{2 V_{d c} J_{n}(k \pi m)}{k \pi} \sin \left(\frac{n \pi}{2}\right)\right|
$$

when $k=1,2, \ldots, \infty$ and $n= \pm 1, \pm 2, \ldots, \infty$

Fig. 6.2 illustrates the major harmonic power density spectrum of the current output of the inverter. The dc-link voltage $V_{d c}$ is $210 \mathrm{~V}$, inverter-side current ripple is $16.4 \% I_{r e f}$ 


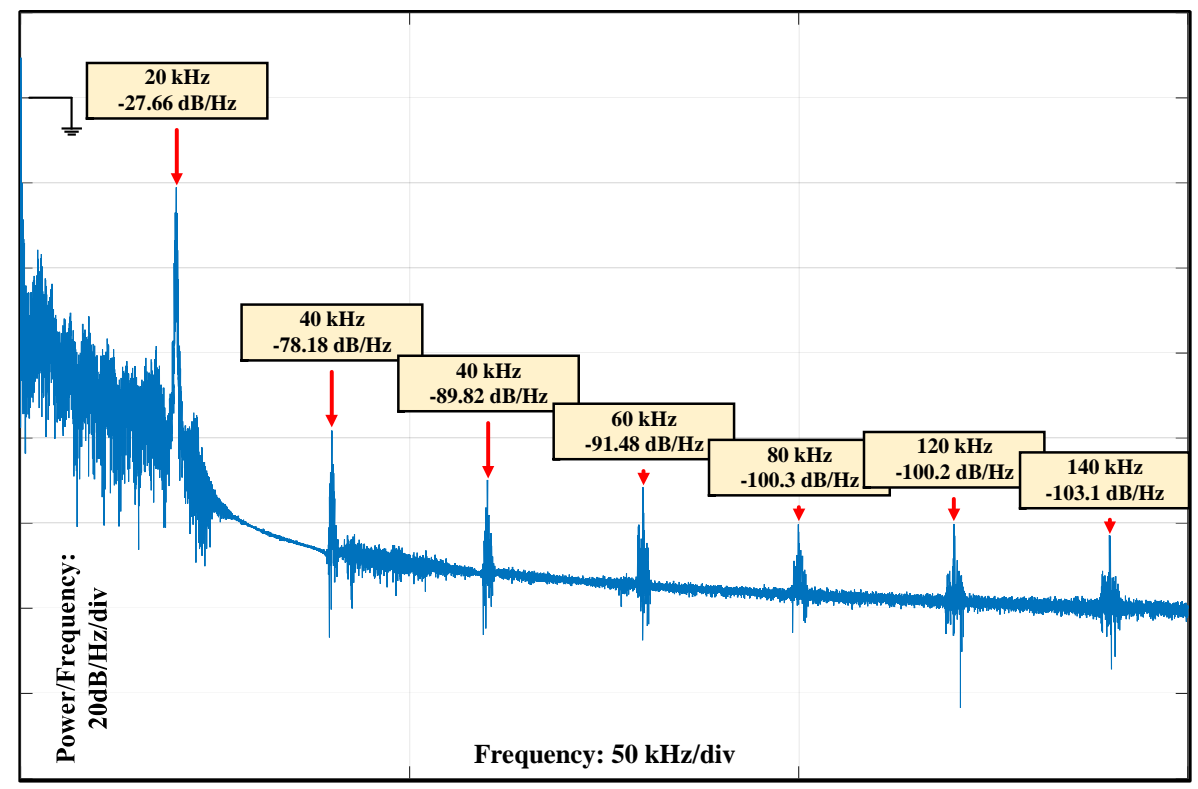

Figure 6.2: Main harmonic current power density spectrum of inverter using unipolar modulation.

( $I_{r e f}$ is fundamental peak current), and the switching frequency $f_{s}$ is $20 \mathrm{kHz}$. The modulation index, $m$, is assumed to be 0.9 . It is clear that the harmonics around the switching frequency and then multiples of the switching frequency are the topmost harmonics of the inverter output current. Consequently, the paralleled trap $L_{f} C_{f}$ is mainly limited by the harmonics around the switching frequency and the grid-side inductor $L_{21}$ is limited by double the switching frequency [222].

Assuming that the grid is an ideal sinusoidal voltage source, the transfer functions $i_{i}(s) / v_{i}(s)$ can be calculated as

$$
\begin{aligned}
& G_{u_{i} \rightarrow i_{i}}(s)=\left.\frac{i_{i}(s)}{v_{i(s)}}\right|_{v_{g}(s)=0} \\
& =\frac{\left(L_{21}+L_{f}\right) C_{f} s^{2}+1}{\left(L_{11} L_{21} C_{f}+\left(L_{11}+L_{21}\right) L_{f} C_{f}\right) s^{3}+\left(L_{11}+L_{21}\right) s}
\end{aligned}
$$

and the transfer functions $i_{g}(s) / v_{i}(s)$ of $L L C L$ filter can be expressed as 


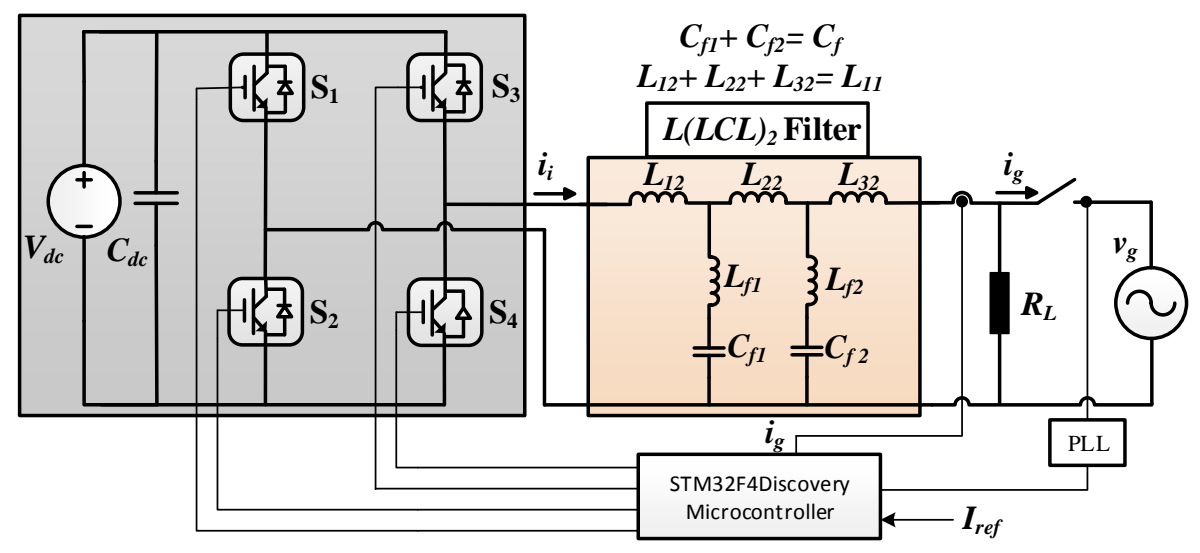

Figure 6.3: Proposed $L(L C L)_{2}$ system.

$$
\begin{aligned}
& G_{u_{i} \rightarrow i_{g}}(s)=\left.\frac{i_{g}(s)}{v_{i(s)}}\right|_{v_{g}(s)=0} \\
& =\frac{L_{f} C_{f} s^{2}+1}{\left(L_{11} L_{21} C_{f}+\left(L_{11}+L_{21}\right) L_{f} C_{f}\right) s^{3}+\left(L_{11}+L_{21}\right) s}
\end{aligned}
$$

\subsection{Proposed $L(L C L)_{2}$ Filter}

In this chapter, a new topology of the $L L C L$ filter structure is proposed, as also illustrated in Fig. 6.3, where the inverter-side inductance of the $L L C L$ filter is separated into three sections in order to allow inserting resonant traps in between them. Also, the resonant capacitor of the traditional $L L C L$ filter is divided into two capacitors to produce a new resonant branch at double the switching frequency. Consequently, the grid-side inductance of the $L L C L$ filter $\left(L_{21}\right)$ can be removed. As a result, since the total amount of the capacitor does not change, the capacitive reactive power at rated load will remain constant. Compared to the conventional $L L C L$ filter-based system, not only does the additional trap inserted between grid-side inductance not add to the control difficulties of the system, but also it reduces the size of the electromagnetic part of the system, which leads to a more efficient low pass filter. Considering $\mathrm{A}(\mathrm{s})$ and $\mathrm{M}(\mathrm{s})$ definitions as below 


$$
A(s)=\frac{Z_{2}(s) Z_{f 2}(s)}{Z_{2}(s)+Z_{f 2}(S)}, M(s)=\frac{Z_{2}(s)}{Z_{f 2}(s)}+1
$$

where $Z_{1}(s)=s L_{12}, Z_{2}(s)=s L_{22}=s L_{32}, Z_{f 1}(s)=s L_{f 1}+1 / s C_{f 1}$ and $Z_{f 2}(s)=$ $s L_{f 2}+1 / s C_{f 2}$. The transfer functions $i_{1}(s) / v_{i}(s)$ and the transfer functions $i_{g}(s) / v_{i}(s)$ of $L(L C L)_{2}$ filter can be, respectively, calculated as

$$
\begin{aligned}
G_{u_{i} \rightarrow i_{i}}(s) & =\left.\frac{i_{i}(s)}{v_{i(s)}}\right|_{v_{g}(s)=0} \\
= & \frac{A+Z_{2}+Z_{f 1}}{\left(A+Z_{2}+Z_{1}\right) Z_{f 1}+\left(A+Z_{2}\right) Z_{1}} \\
G_{u_{i} \rightarrow i_{g}}(s)= & \left.\frac{i_{g}(s)}{v_{i(s)}}\right|_{v_{g}(s)=0} \\
= & \frac{Z_{f 1}}{Z_{2}\left(M+Z_{f 1}\left(M+Z_{2}\right)+1\right)+M Z_{1} Z_{f 1}}
\end{aligned}
$$

Fig. 6.4 shows bode plots of the transfer function $i_{g}(s) / v_{i}(s)$ of both the $L(L C L)_{2}$ filter and the $L L C L$ filter, while all the other parameters are the same, except inductances of the traps. Also, $L_{11}$ in the $L L C L$ filter is divided into three parts and $C_{f}$ is divided into two capacitors. Fig. 6.5 presents bode plots of the transfer function $i_{i}(s) / v_{i}(s)$ with aforementioned parameters. The figures help in verifying that all the requirements are satisfied with the design.

It is clear that within half of the switching frequency range, the $L(L C L)_{2}$ filter has a nearly identical frequency response pattern of an $L L C L$ filter, and both resonant frequencies match the resonant frequency criteria of the low-pass filters for having a stable system. That is to say, compared to an ordinary $L L C L$ filter, the additional $C_{f 2} L_{f 2}$ branch of the $L L C L$ filter does not bring any further control worries. Fig. 6.6 shows the size of the total impedance of the $L(L C L)_{2}$ filter when frequency varies from 0 to $20 \mathrm{kHz}$. It can be seen that the impedance of the proposed filter is lower than the $L L C L$ filter, except around resonant frequencies of the filters. 


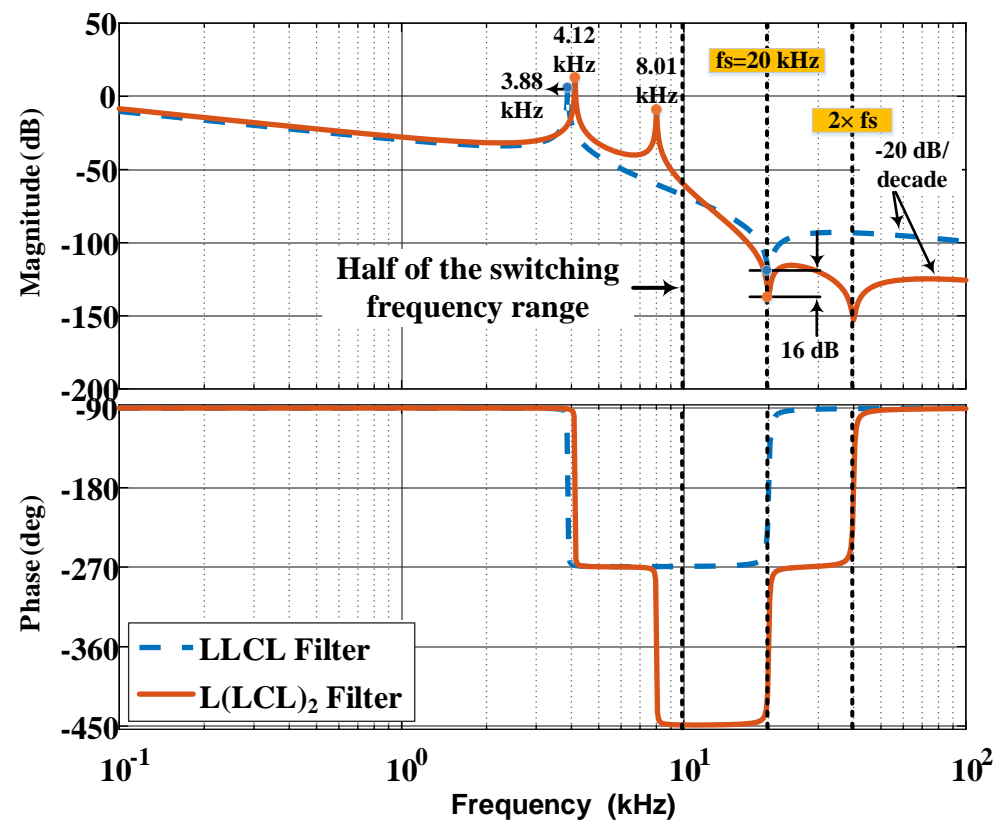

Figure 6.4: Bode plots of transfer functions $i_{g}(s) / v_{i}(s)$.

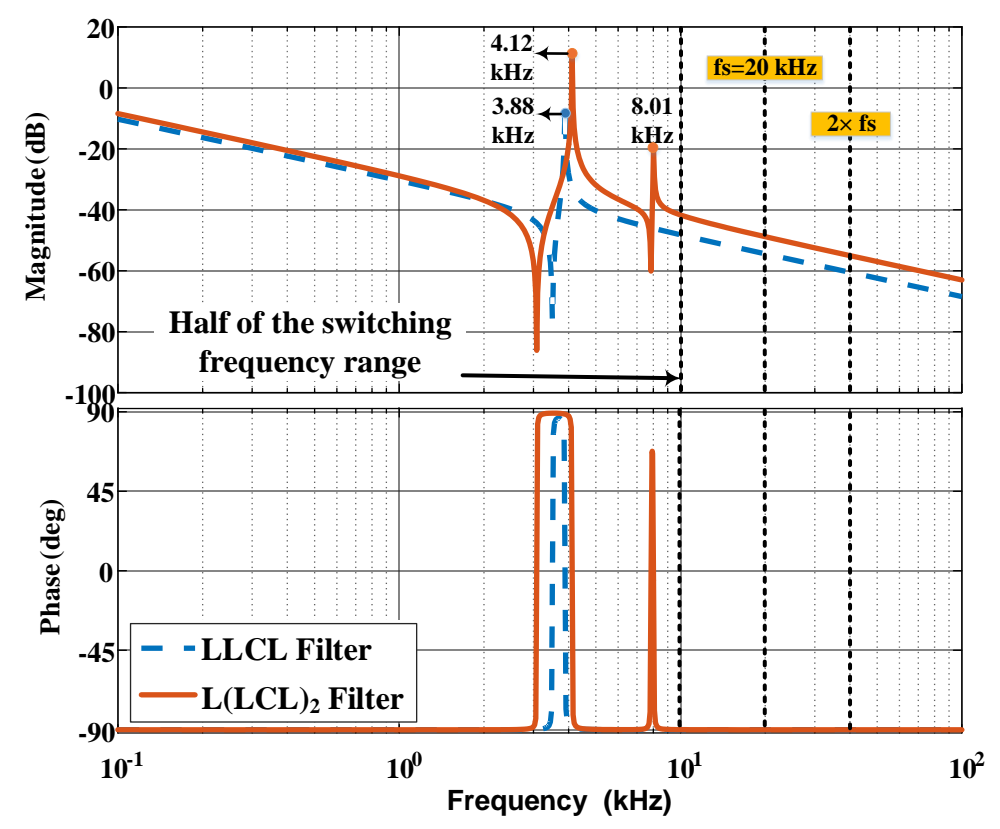

Figure 6.5: Bode plots of transfer functions $i_{i}(s) / v_{i}(s)$.

\subsubsection{Analysis of ripple current}

The time average value of the output voltage of the inverter, $v_{A V}$, can be assumed constant during the switching period $T_{s}$, if the switching frequency, $f_{s}$, is greater than the grid 


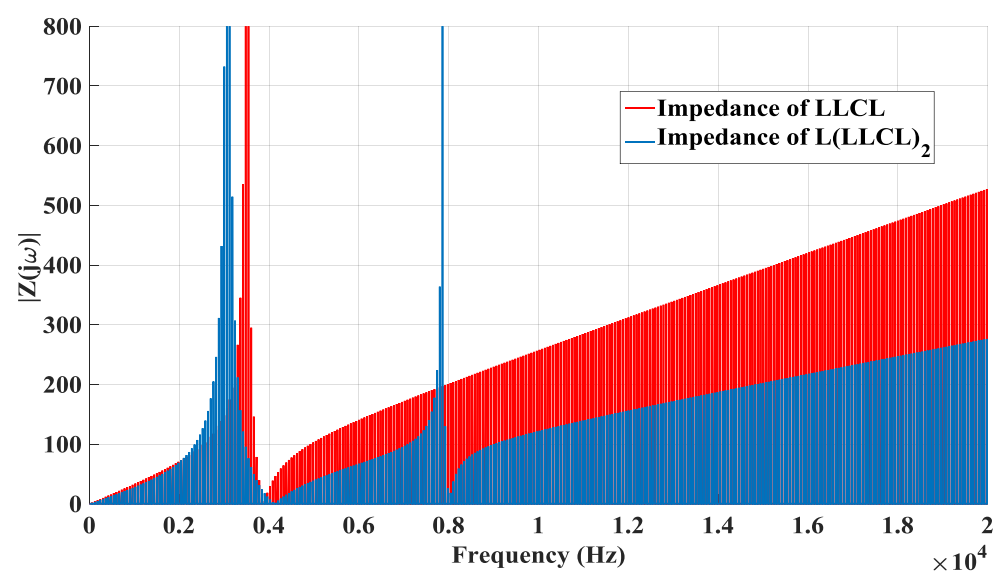

Figure 6.6: Size of the impedance of the $L L C L$ and the $L(L C L)_{2}$ filters for different frequencies.

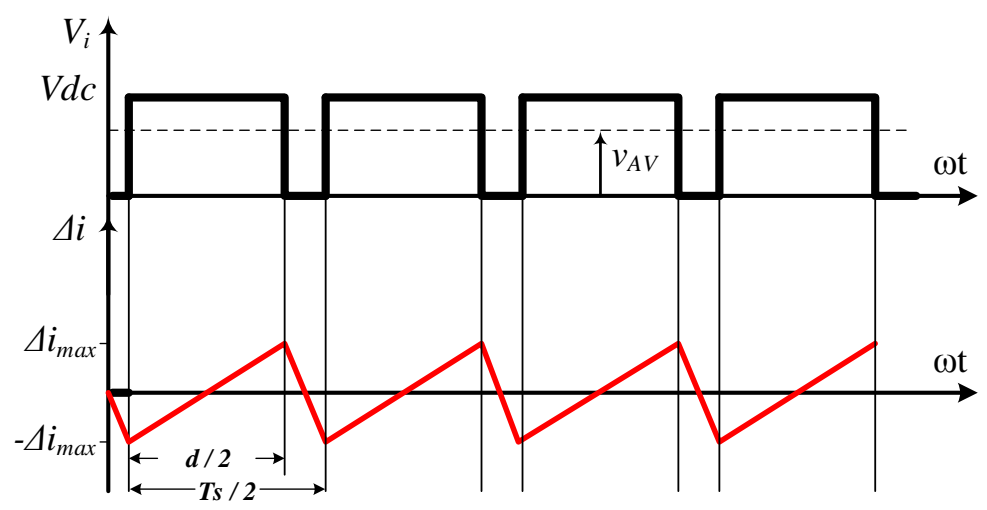

Figure 6.7: Voltage and the current output waveform of typical single-phase full-bridge inverters.

frequency, $f_{0}$.

Hence, the current of the filter inductor of the grid-tied single-phase full-bridge inverters has a symbolic curve for the duration of any period, as illustrated by the lower waveform in Fig. 6.7. In this situation, $\triangle i_{p p}$, the peak-to-peak value of the filter inductor current, which is generated by the unipolar PWM switching, can be calculated as [223]

$$
\triangle i_{p p}=2 \triangle i_{\text {max }}=\frac{V_{d c}-v_{A V}}{L} \frac{d}{2} T_{s}
$$

where $L$ is the inverter side inductor of any kind of filter (including $L C L, L L C L, L(L C L)_{2}$, 
etc.). Moreover, grid voltage $v_{g}$ is supposed to be a clean sinusoidal waveform. To make the analysis simpler, the fundamental component of the grid current in Fig.6.3 is pretended to be zero. Consequently, the fundamental component of the voltage appears on the filter inductor is similarly zero as

$$
v_{L}=v_{i}-v_{g}=0
$$

Therefore, when $0<\omega t<\pi$, equations (6.12) and (6.13) can be calculated as

$$
\begin{gathered}
v_{A V}(\omega t)=d(\omega t) V_{d c}, v_{g}(\omega t)=m \cdot V_{d c} \sin (\omega t) \\
d(\omega t)=m \cdot \sin (\omega t)
\end{gathered}
$$

and during $0<\omega t<\pi$, the peak-to-peak value of the current of the filter inductor will be [224]

$$
\triangle i_{p p}(\omega t)=\frac{V_{d c} T_{s}}{4 L}\left(1-m^{2} \cdot \sin ^{2}(\omega t)\right)
$$

\subsubsection{Parameter Design of the $L(L C L)_{2}$ Filter}

Some limitations were introduced by $[14,214]$ to be considered when designing the $L C L$ and $L L C L$ filters, which could be used in the $L(L C L)_{2}$ filter.

1. The total capacitive reactive power at rated load should be less than $5 \%$ of the nominal power and capacitors are limited by this constraint.

2. The total inductance is limited by the voltage drop for the duration of operation (less than 10\%). If not, the dc-link voltage will be required to be higher to guarantee controllability of current, which will cause greater losses in switching devices.

3. The range of the resonant frequency ought to be bigger than ten times the line frequency or one-sixth of the switching frequency (whichever is higher) and onehalf of the switching frequency, with the intention of keeping away from control 
and stability problems, which possibly caused by resonance in the upper and the lower parts of the harmonic spectrum.

4. The inverter-side inductor, $L_{12}$, is constrained by the maximum ripple current requirement (normally $\leq 40 \%$ ).

5. Considering IEEE 519-2014, the harmonics greater than the $35^{\text {th }}$ should be less than $0.3 \%$ of the rated fundamental current, if the short-circuit current of the system is less than 20 times of the fundamental grid-side current.

Considering the mentioned constraints, the $L(L C L)_{2}$ filter can be designed by using the following steps

1. With the intention of meeting a particular requirement for current ripple, the inductance can be designed from

$$
\frac{V_{d c}}{4 f_{s}\left(\alpha_{1} I_{r e f}\right)} \geq L_{11} \geq \frac{V_{d c}}{4 f_{s}\left(\alpha_{2} I_{r e f}\right)}
$$

where, $I_{r e f}$ is the rated reference peak current, $\alpha_{1}$ and $\alpha_{2}$ are the inverter-side current ripple ratio, which generally have the value of $15 \%$ and $40 \%$, respectively. This inductance is the amount of total inductance of the filter $\left(L_{11}=L_{12}+L_{22}+L_{32}\right)$, while $L_{12}=K \times L_{11}(33 \%<K<60 \%)$ and $L_{22}=L_{32}=\left(L_{11}-L_{12}\right) / 2$.

2. By selecting the absorption of reactive power, while the system is operating in rated conditions, the capacitor value can be determined.

$$
C_{f}(\text { Total })=x C_{b}
$$

where $x$ is the absorbed reactive power percentage at full-load conditions $(x<1)$. The total capacitor number is limited by (6.17)

$$
C_{\text {max }}=\frac{5 \% P_{\text {rated }}}{V_{g}^{2} \omega_{0}}
$$


Then the capacitor of each branch is almost half of the total capacitor in the $L L C L$ filter.

3. The resonant frequency of the $L_{f 1} C_{f 1}$ and the $L_{f 2} C_{f 2}$ circuits are at the switching frequency and the double of the switching frequency, respectively. Thus, $L_{f 1}$ and $L_{f 2}$ can be calculated by

$$
\frac{1}{\sqrt{L_{f 1} C_{f 1}}}=\omega_{s 1}, \frac{1}{\sqrt{L_{f 2} C_{f 2}}}=\omega_{s 2}
$$

where, $\omega_{s 1}$ is the switching frequency and $\omega_{s 2}$ is twice the switching frequency.

4. In $L L C L$ filters, grid-side inductance, $L_{21}$, is mostly used to reduce each harmonic around twice the switching frequency down to $0.3 \%$. For an $L(L C L)_{2}$ filter, due to the additional $L_{f 2} C_{f 2}$ branch, the current harmonics near the double of the switching frequency fulfill the IEEE 519-2014 standard with far more ease. Therefore, $L_{21}$ is replaced by a portion of $L_{11}$.

5. The resonant frequency can be calculated by setting the dominator of equations (6.9) to zero, after replacing " $s "$ with " $j \omega$ ". If it does not satisfy the requirement 3 , absorbed reactive power can be changed, then return to step 2. Otherwise, the tolerable current ripple can be adjusted again, then return to step 1.

6. The quality factor of each resonant circuit should be $10 \leq Q \leq 50$, that can be calculated as

$$
Q=\frac{1}{R_{f}} \sqrt{\frac{L_{f}}{C_{f}}}
$$

where $R_{f}$ is the gaped equivalent resistance of the inductors in the resonant branches $\left(L_{f 1}\right.$ and $\left.L_{f 2}\right)$.

The most important limitations, such as the voltage drop across the inductor, the capacitive reactive power, and the amplitude of the harmonic currents, should be considered, while the design procedure is iterative with the $L(L C L)_{2}$ parameter values adjusted. The algorithm for designing the $L(L C L)_{2}$ filter is shown in Fig. 6.8. 


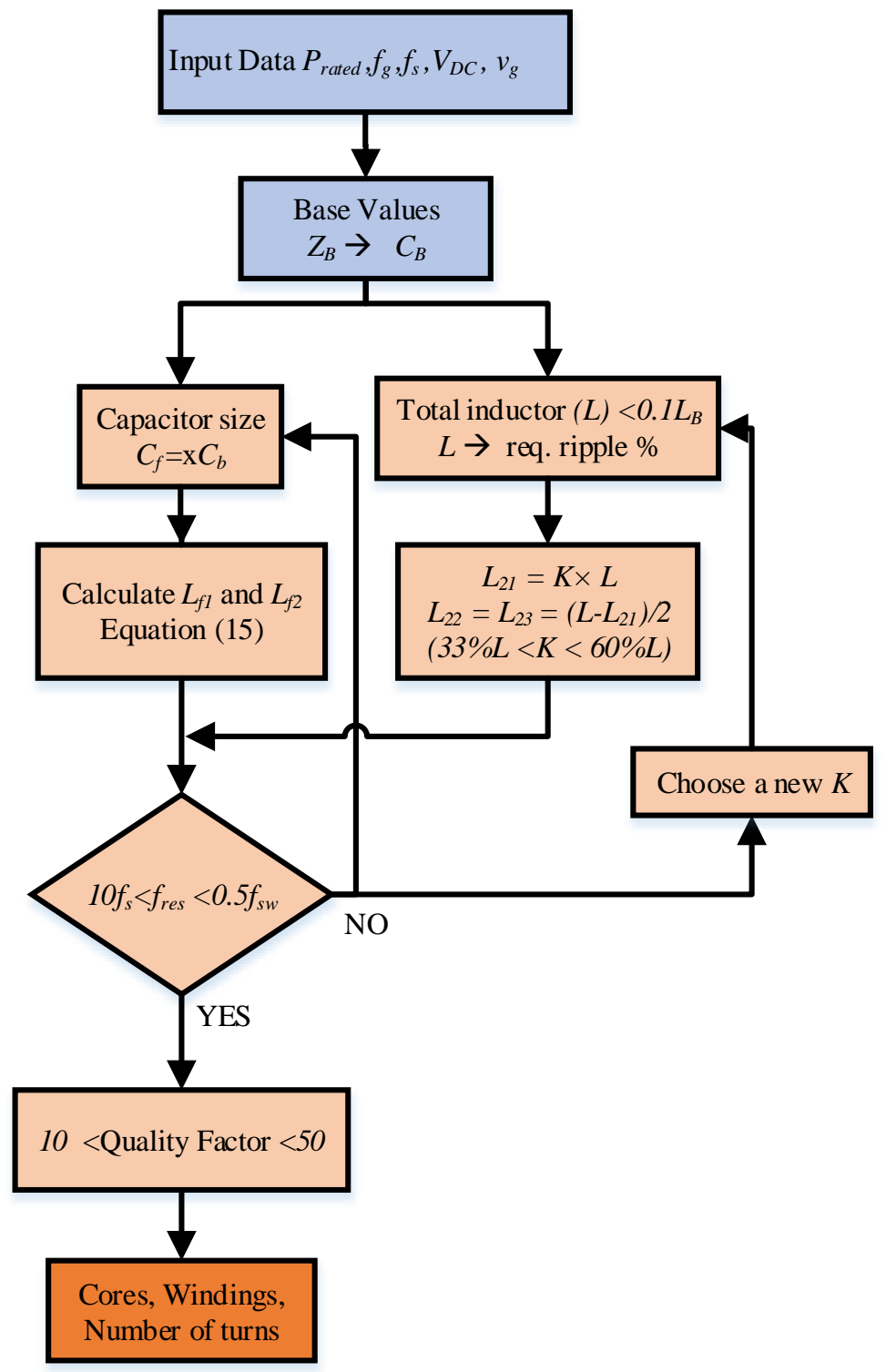

Figure 6.8: Flowchart of the parameter design procedure of $L(L C L)_{2}$ filter.

\subsubsection{Design example}

Once designing a high-order filter for power inverters, the base values of the total impedance, inductance, and capacitance should be defined as

$$
Z_{b}=\frac{v_{g}}{P_{\text {rated }}}, L_{b}=\frac{Z_{b}}{\omega_{b}}, C_{b}=\frac{1}{\omega_{b} Z_{b}}
$$


where $\omega_{b}$ is the grid frequency and $P_{\text {rated }}$ is the rated active power of the inverter. Considering the constraints addressed in section II(A), and under the condition of that $f_{s}=20 \mathrm{kHz}$, $V_{d c}=210 \mathrm{~V}, P_{\text {rated }}=700 \mathrm{~W}$, grid phase to ground voltage is $120 \mathrm{~V} / 60 \mathrm{~Hz}$. Then, the attenuation of the current harmonics focused on the design processes of the $L(L C L)_{2}$ filter, which can be derived as

1. By assuming the $7.7 \%$ impedance for the inverter-side inductor, $L_{11}$ is selected to be $4.2 \mathrm{mH}$. For an $L L C L$ filter, $L_{21}$ mostly designed on the objective to decrease each harmonic around the double of the switching frequency down to $0.3 \%$, but for the $L(L C L)_{2}$ filter, owing to the $L_{f 2} C_{f 2}$ resonate circuit, the twice of the switching frequency harmonics are approximately eliminated. Therefore, the calculated inductance for inverter-side inductance of the traditional $L L C L$ filter is split into three smaller inductances. The first part has a value of $L_{12}=2.2 \mathrm{mH}$ (about $53 \%$ of calculated inverter-side inductance of the conventional $L L C L$ filter), then $L_{22}=1 \mathrm{mH}$ and $L_{32}=1 \mathrm{mH}$. The inverter-side inductor $L_{12}$ should fulfill the requirements of (6.15). In this case, the value of inverter-side current ripple is $16.40 \%$, which is in the range between $10 \%$ and $40 \%$.

2. To achieve the limitation of the maximum absorbed reactive power at rated conditions, the total capacitance $\left(C_{f 1}+C_{f 2}\right) \leq 0.05 C_{b}$. Hence, the capacitor value is designed to be $C_{f 1}+C_{f 2}=2 \mu F$ in order to limit the reactive power to $1.55 \%$. This value can be increased to the limit of $5 \%$, if some of the constraints cannot be met.

3. The grid-side inductor of $L_{21}$ is removed in the $L(L C L)_{2}$ filter, but $L_{22}$ and $L_{23}$ are two sections of the split inductor and both of them have the value of $1 \mathrm{mH}$.

4. The consequent resonance frequency is $3.88 \mathrm{kHz}$ for the $L L C L$, and the $L(L C L)_{2}$ filter has two resonant frequencies at $4.12 \mathrm{kHz}$ and $8.01 \mathrm{kHz}$. Both resonant fre- 
Table 6.1: Size Estimation of $L L C L$ and $L(L C L)_{2}$ Filters

\begin{tabular}{c||c||c}
\hline Components & $\begin{array}{c}\text { Energy Stored in } \\
\text { the Components }(j)\end{array}$ & $\begin{array}{c}\text { Size of } \\
\text { the components }\left(\mathrm{m}^{3}\right)\end{array}$ \\
\hline \hline$L_{11}+L_{12}$ & 0.0919 & $0.0496 \times 10^{-3}$ \\
$L_{f}$ & $1.29 \times 10^{-7}$ & $0.6966 \times 10^{-10}$ \\
$C_{f}$ & 0.0144 & $0.016 \times 10^{-3}$ \\
$L_{12}+L_{22}+L_{32}$ & 0.0715 & $0.0386 \times 10^{-3}$ \\
$L_{f 1}+L_{f 2}$ & $8.09 \times 10^{-8}$ & $4.3686 \times 10^{-11}$ \\
$C_{f 1}+C_{f 2}$ & 0.0144 & $0.016 \times 10^{-3}$
\end{tabular}

quencies of the $L(L C L)_{2}$ are between one half of the switching frequency and one sixth of the switching frequency.

5. The quality factor of resonant branches is chosen to be 50 , and the equivalent resistor value of $R_{f 1}$ is $0.16 \Omega$ and $R_{f 2}$ is $0.08 \Omega$.

\subsubsection{Size Estimation of $L L C L$ and $L(L C L)_{2}$ Filters}

Exact size approximation is challenging because of different manufacturing processes and design parameters. To generate an initial estimate, however, A number of methods were proposed for the size estimation of filter inductors and capacitors based on peak energy requirements. Although final recognitions probably will not obey this linear scaling, the component choice for inductor and capacitor volume with rated energy are accurate. The scaling factors are $1.11 \mathrm{~m}^{3} / \mathrm{kj}$ for inductive component and $0.54 \mathrm{~m}^{3} / \mathrm{kj}$ for capacitive component [215], a ratio of approximately 2 to 1 , where $j$ stands for joules. In order to give an estimate of stored energy and size of the components, using the relationship $E_{c}=\frac{1}{2} C V^{2}$ and $E_{L}=\frac{1}{2} L I^{2}$, Table 6.1 is generated. By looking at Table 6.1 it is clear that the total size of the $L L C L$ filter is $0.0656 \times 10^{-3} \mathrm{~m}^{3}$ and the total size of the $L(L C L)_{2}$ filter is $0.0546 \times 10^{-3} \mathrm{~m}^{3}$. Therefore, by implementing the $L(L C L)_{2}$ filter the size of the filter is reduced by the factor of $16.8 \%$. 


\subsubsection{Efficiency comparison between the $L L C L$ and $L(L C L)_{2}$ Filters}

The proposed $L(L C L)_{2}$ filter can be more attractive than an $L L C L$ filter as the interface between the inverter and the grid requires reduced copper and magnetic materials. The power losses of the filters can be separated into:

1) High-frequency passive damping loss, which can be obtained as follows

$$
P_{d}(\text { ripple })=\frac{1}{2}\left|I_{A M}\left(n, k_{1}\right)\right|^{2} \times R_{f 1}+\left|I_{A M}\left(n, k_{2}\right)\right|^{2} \times R_{f 2}
$$

where $n= \pm 1, \pm 3, \pm 5, k_{1}=1$ and $k_{2}=2$.

Passive Damping (PD) techniques use a resistance connected in series with the capacitor in order to attenuate the $L C$ branch resonance at the cost of curtailing efficiency [225]. Lossless Active Damping (AD) methods with virtual resistors have been widely explored to suppress these oscillations. By using this technique, a resistance damping characteristic emulated in the lossless method, and no resistance is physically connected to the circuit [226]. Therefore, when using active damping method, $P_{d}$ can be set to zero.

2) Power losses in inductors, can be separated into $P_{\text {core }}$ (core loss) and $P_{\text {copper }}$ (copper loss)

$$
P_{\text {core }}=k\left(f^{\alpha} B^{\beta} V_{e}\right)
$$

where $k, \alpha$, and $\beta$ are material parameters generally found by curve fitting; $B$ is the flux density, in Tesla and $V_{e}$ is the magnetic core volume, in $\mathrm{cm}^{3}$.

$$
P_{\text {copper }}=I_{f} R_{d c}^{2}+I_{a c} R_{a c}^{2}
$$

where $I_{f}$ is the RMS value of the fundamental frequency current component and $I_{a c}$ is the RMS value of the ac-ripple current component. it can be assumed that $R_{a c}=R_{d c}$ in this study. The power loss and efficiency values of the $L(L C L)_{2}$ filter and the $L L C L$ filter for both PD and AD methods are presented in Table 6.2. It can be seen that the efficiency 
Table 6.2: Calculated Power Losses And Efficiency of The $L(L C L)_{2}$ Filter and the $L L C L$ Filters Based on Nominal Output Power of 700w.

\begin{tabular}{c|c|c}
\hline & $L L C L$ & $L(L C L)_{2}$ \\
\hline \hline$P_{d}$ & $0.0064 w$ & $0.0345 w$ \\
$P_{\text {copper }}$ & $3.4432 w$ & $2.7593 w$ \\
$P_{\text {core }}$ & $1.8238 w$ & $1.3015 w$ \\
Total Loss(PD) & $\mathbf{5 . 2 7 3 5 w}$ & $\mathbf{4 . 0 9 5 3 w}$ \\
Total Loss(AD) & $\mathbf{5 . 2 6 7 1 w}$ & $\mathbf{4 . 0 6 0 8 w}$ \\
Efficiency(PD) & $99.247 \%$ & $99.415 \%$ \\
Efficiency(AD) & $99.248 \%$ & $99.420 \%$
\end{tabular}

is increased by $0.169 \%$ (for PD method) and $0.173 \%$ (for AD method) compared to that of the $L L C L$ filter.

The total loss of the $L L C L$ and $L(L C L)_{2}$ filters are measured in our hardware prototype by means of a LaCroy WaveRunner 64Xi, $600 \mathrm{MHz}$ Oscilloscope. The measured output power was 691.55 watts for the $L L C L$ and 692.74 watts for $L(L C L)_{2}$ filter. The input power of both filters (which is the output power of the VSC) was 697.27 watts. The values in Table 6.2 are based on calculation, but the measured values of efficiencies are 99.17\% and $99.35 \%$ for $L L C L$ and $L(L C L)_{2}$, respectively. Therefore, the measured total power losses for the $L L C L$ filter and the $L(L C L)_{2}$ filter are higher in value by a factor of $8.54 \%$ and $10.63 \%$, respectively, comparing to calculated losses.

\subsubsection{Optimization of The $L(L C L)_{2}$ Parameters}

Frequent trial and error in the design procedure increase the need to conduct an optimization of $L(L C L)_{2}$ filter on Matlab software, due to the connection between the filter parameters and the device performance indexes, the optimization is based on the total harmonic distortion (THD). Although, some useful trial and error solutions with computer programs can be achieved, for instance in [227]. Changes of several performance indexes could be caused by the variation of one of the filter parameters. For building an accu- 
rate THD model, the harmonics caused by power switches and the control-loop should be taken into consideration. The current harmonics provided by closed-loop transfer functions of $L(L C L)_{2}$ filter can be expressed as follows [228] where $v_{i}(t)$ is calculated by (6.1) and the open-loop transfer function of $L(L C L)_{2}$ filter , $\mathrm{G}(\mathrm{s})$, can be calculated by (6.9). According to [228], the goal of optimization is to find the minimum values of $L_{12}, L_{22}$, and $L_{32}$ on the basis of the allowable THD. The objective function for Genetic Algorithm (GA) optimization can be expressed as

$$
\min f(x)=\left(2+\frac{1}{r}\right) L_{22}\left(x=\left[L_{22}, r, C_{f 1}, C_{f 2}\right]\right)
$$

when $L_{22}=L_{23}$ and $r=L_{22} / L_{12}$. To leave a certain margin, the restrictive value of THD can be chosen as $3 \%$ (instead of 5\%). GA is an effective method to find the optimal solution. The constraint can be expressed as $L_{12}, L_{22}$, and $L_{32}$ on the premise of the allowable THD. The objective function for GA optimization can be expressed as

$$
T H D(x)=3 \%
$$

The parameter variety can be obtained from Section III (B). On this basis, the allowed range of $L_{22}, r, C_{f 1}$ and $C_{f 2}$ are $0.311 \mathrm{mH} \leq L_{22}=L_{32} \leq 1.505 \mathrm{mH}, 0.07 \leq \mathrm{r} \leq 1.19$, $0.5 \mu F \leq C_{f 1}, C_{f 2} \leq 3 \mu F$. With the initial values of [0.908 $\mathrm{mH}, 0.63,1.75 \mu F, 1.75$ $\mu F]$, the optimized result is $[0.918 \mathrm{mH}, 0.42,0.935 \mu F, 0.941 \mu F]$. In the real world, capacitors have a series of fixed values. The one which is closest to $0.935 \mu F$ and 0.941 $\mu F$ is $1 \mu F$. Therefore, the values of the $C_{f 1}$ and the $C_{f 2}$ are chosen as $1 \mu F$. When the fixed value of $C_{f 1}$ and $C_{f 2}$ are considered, the parameters of $L(L C L)_{2}$ filter are chosen as [1.02 $\mathrm{mH}, 0.43,1 \mu F, 1 \mu F]$. Compare to step by step method, these values have $2 \%$, $2.3 \%, 0 \%$ and $0 \%$ error, respectively. These errors are within an acceptable range. 


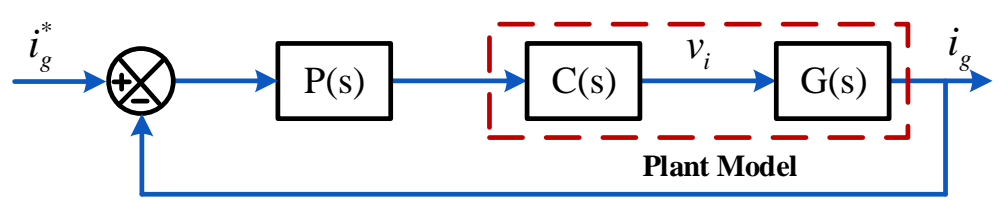

Figure 6.9: Block diagram of the current control system.

\subsubsection{Close-Loop Stability Analysis}

Many techniques are used for the analysis of the stability of a system. A popular technique is analysis of the eigenvalues to study the system stability [229,230], which is explained in this section. Fig. 6.9 shows the typical block diagram of a current controller, where $\mathrm{P}(\mathrm{s}), \mathrm{C}(\mathrm{s})$, and $\mathrm{G}(\mathrm{s})$ are the model of the controller, inverter, and the proposed filter, respectively. A conventional PI controller is adopted as $\mathrm{P}(\mathrm{s})$ in order to achieve the specified dynamics, i.e.

$$
P(s)=\frac{\left(k_{p} s+k_{I}\right)}{s} .
$$

where $k_{p}=1$ is the proportional gain and $k_{I}=100$ is integral gain. These values are designed and tuned by the trial and error method. The system time delays due to discrete operation and phasor PWM blocks are neglected, i.e., $C(s)=1$ and $G(s)$ is calculated by (6.9), thus, the closed-loop transfer function of the current control system can be written as

$$
H(s)=\frac{i_{g}(s)}{i_{g}^{*}(s)}=\frac{P(s) G(s)}{1+P(s) G(s)}
$$

The eigenvalues of (6.27) determine the dynamics of the closed-loop system. Herein, the stability of the proposed system, with respect to the variation in system matrix parameters, is studied. This study is based on a $700 \mathrm{w}$ laboratory prototype, in which parameters are summarized in Table 6.3. The eigenvalues of the system at this operating point are $\lambda_{1,2}=-119.04 \pm j 98.16, \lambda_{2,3}=-288.88 \pm j 25872.9$, and $\lambda_{4,5}=-1401.15 \pm j 50313.5$. 
Table 6.3: Designed Parameters for Experimental Setup

\begin{tabular}{c||c||c}
\hline Elements & Parameters & Values \\
\hline \hline Inverter & dc-link voltage $\left(V_{d c}\right)$ & $210 \mathrm{~V}$ \\
& Switching frequency $\left(f_{s}\right)$ & $20 \mathrm{kHz}$ \\
& Rated power $\left(P_{r a t e}\right)$ & $700 \mathrm{w}$ \\
\hline AC Grid & Grid phase voltage $\left(v_{g}\right)$ & $120 \mathrm{~V}$ \\
& Grid frequency $\left(f_{0}\right)$ & $60 \mathrm{~Hz}$ \\
\hline$L(L C L)_{2}$ filter & Inverter side inductor $\left(L_{12}\right)$ & $2.2 \mathrm{mH}$ \\
& Grid side inductors $\left(L_{22}=L_{32}\right)$ & $1 \mathrm{mH}$ \\
& Resonant circuit inductor $\left(L_{f 1}\right)$ & $63.3 \mu \mathrm{H}$ \\
& Resonant circuit inductor $\left(L_{f 2}\right)$ & $15.83 \mu \mathrm{H}$ \\
& Resonant circuit capacitors $\left(C_{f 1}=C_{f 2}\right)$ & $1 \mu \mathrm{F}$ \\
& Equivalent resistance of the inductor $\left(R_{f 1}\right)$ & $0.16 \Omega$ \\
& Equivalent resistance of the inductor $\left(R_{f 2}\right)$ & $0.08 \Omega$ \\
\hline \multirow{2}{*}{$L L C L f i l t e r$} & Converter side inductor $\left(L_{11}\right)$ & $4.2 \mathrm{mH}$ \\
& Grid side inductor $\left(L_{21}\right)$ & $1.2 \mathrm{mH}$ \\
& Resonant circuit inductor $\left(L_{f}\right)$ & $31.67 \mu \mathrm{H}$ \\
& Resonant circuit capacitor $\left(C_{f}\right)$ & $2 \mu \mathrm{F}$ \\
& Equivalent resistance of the inductor $\left(R_{f}\right)$ & $0.11 \Omega$ \\
\hline
\end{tabular}

The real parts of all six eigenvalues are negative, which signifies the linearized system's stability. However, the stability of the overall system cannot be guaranteed for all values of the filter parameters. Thus, for stability analysis, the eigenvalues of the system are plotted versus the variation of one parameter at a time, while the other nominal parameters remain constant.

It is worth mentioning that the range oscillation of all the parameters corresponds to the step by step design procedure in Section III. Furthermore, in transfer function (6.27), the system eigenvalues remain almost constant with $\pm 20 \%$ variations of the $L_{f 1}$ and the $L_{f 2}$. In fact, the variation of the $L_{f 1}$ and $L_{f 2}$ does not affect the stability of the proposed system.

The impacts of $L_{12}$ and $L_{32}$ on the eigenvalues of the system are shown in Fig. 6.10a and Fig. 6.10b, respectively, when $L_{1}$ varies from $1.39 \mathrm{mH}$ to $2.52 \mathrm{mH}$ and $L_{21}$ varies from $0.84 \mathrm{mH}$ to $1.485 \mathrm{mH}$. Also, $L_{22}$ is equal to $L_{32}$, so their variations are depicted together in Fig. 6.10b. 


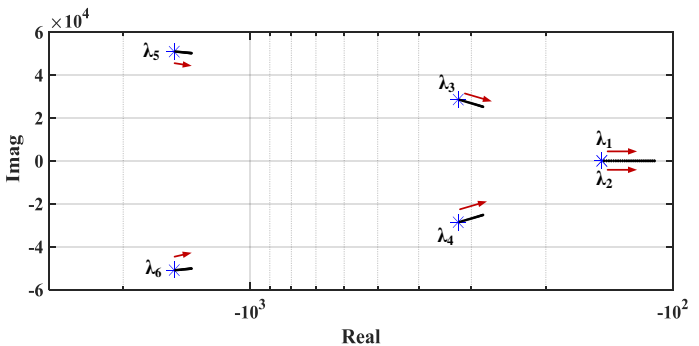

(a)

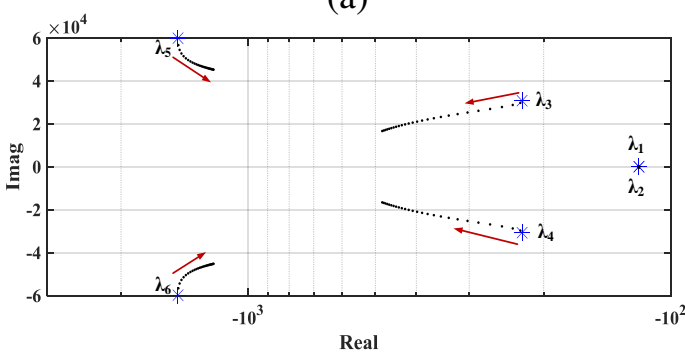

(c)

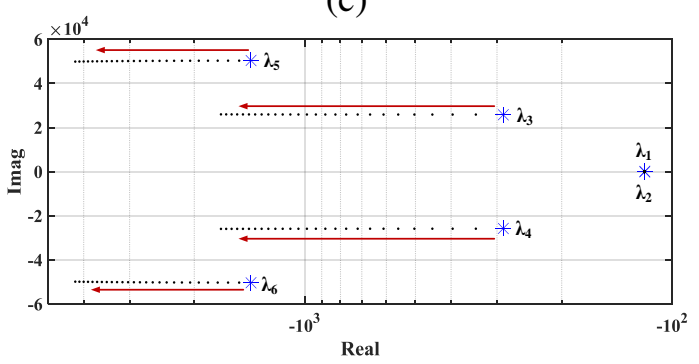

(e)

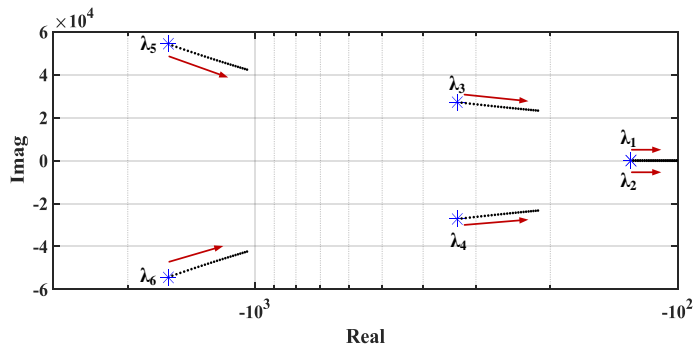

(b)

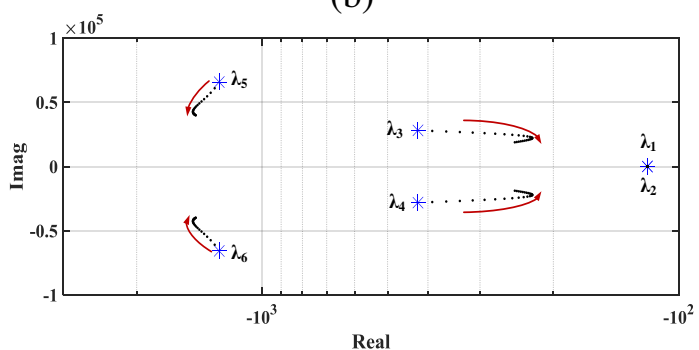

(d)

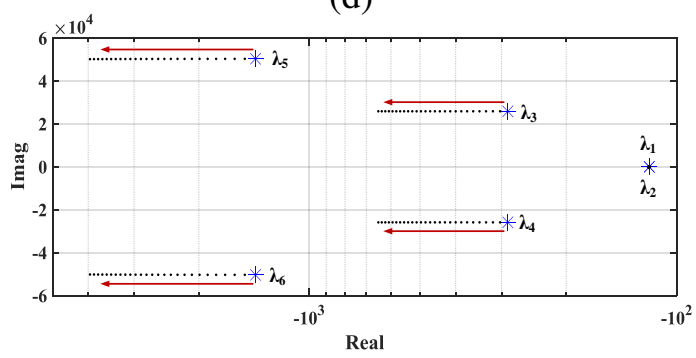

(f)

Figure 6.10: Root locus of the closed-loop transfer function of the system $\left(H_{s}\right)$ as (a) $L_{11}$ varies from $1.39 \mathrm{mH}$ to $2.52 \mathrm{mH}$ (b) $L_{22}$ and $L_{32}$ vary from $0.84 \mathrm{mH}$ to $1.485 \mathrm{mH}$ (c) $C_{f 1}$ varies from $0.5 \mu \mathrm{F}$ to $3 \mu \mathrm{F}(\mathrm{d}) C_{f 2}$ varies from $0.5 \mu \mathrm{F}$ to $3 \mu \mathrm{F}$ (e) $R_{f 1}$ varies from $0.08 \Omega$ to $0.39 \Omega$. (f) $R_{f 2}$ varies from $0.16 \Omega$ to $0.8 \Omega$.

It can be observed that the increase of the inverter-side and the grid-side inductances, displaces all complex eigenvalues toward the vertical axis of the s-plan. Thus, it is predictable that the whole system's response becomes longer and less oscillatory as $L_{12}$ and $L_{32}$ increase.

The variation of system eigenvalues by varying the $C_{f 1}$ and $C_{f 2}$ from $0.5 \mu F$ to $3 \mu F$ are shown in Figs. 6.10c and 6.10d. As it can be seen, the impact of the $C_{f 1}$ and $C_{f 2}$ on the eigenvalues $\lambda_{1}$ and $\lambda_{2}$ are almost negligible. However, Fig. 6.10c shows that the complex eigenvalues $\lambda_{3}, \lambda_{4}, \lambda_{5}$, and $\lambda_{6}$ move toward the real axis, which can reduce the 
system's natural frequencies and increases the damping factor of the system. Fig. 6.10d obviously demonstrates that all eigenvalues stay on the left half of the s-plane for the whole effective range, which presents the stability of the proposed system over the given range.

Figs. 6.10e and 6.10f illustrate the eigenvalues of the proposed system as $R_{f 1}$ and $R_{f 2}$ vary from $0.08 \Omega$ to $0.39 \Omega$ and $0.16 \Omega$ to $0.8 \Omega$, respectively. As can be seen, increasing the resistances of the filter results in moving the real part of eigenvalues $\lambda_{3}, \lambda_{4}, \lambda_{5}$, and $\lambda_{6}$ toward the left, and therefore, a faster response and less oscillatory. However, increase of the resistance increases the power losses of the system, which is usually undesirable.

\subsection{Simulation and Experimental Results}

In order to confirm the effectiveness of the proposed $L(L C L)_{2}$ filter on suppressing the current harmonics, a 700-W prototype of the single-phase full-bridge grid-tied inverter with the "STM32F4" Microcontroller is constructed. In addition, a Matlab Simulink-based study is carried out to assess the experimental analysis. The experimental parameters of the filter are the same as those for simulations listed in Table 6.3.

The experiments are evaluated and investigated under the given conditions of $f_{s}=$ $20 \mathrm{kHz}, V_{d c}=220 \mathrm{~V}, v_{g}=120 \mathrm{~V} / 60 \mathrm{~Hz}, P_{\text {rated }}=700 \mathrm{~W}$, and SPWM strategy is used in the inverter and the dc-link voltage is kept at $220 \mathrm{~V}$.

Case I is the traditional $L L C L$ filter strategy and Case II is the $L(L C L)_{2}$ filter strategy with an extra trap at $40 k H z$. Figs. $6.11-6.15$ show important system measurements that are captured by a LaCroy WaveRunner 64Xi oscilloscope in experimental tests and Matlab Simulink plots.

Figs. 6.11 and 6.12 show the grid side current-voltage waveforms and the power density spectrum of the grid-side current for cases I and II, respectively. Figs. 6.11(b) 


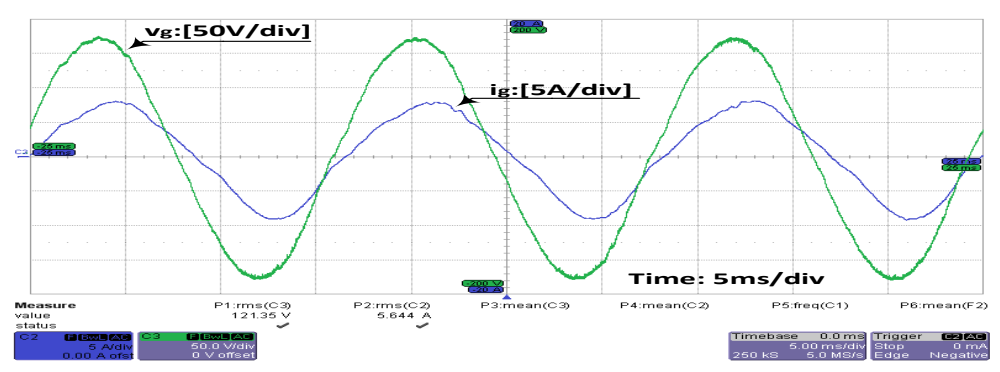

(a)

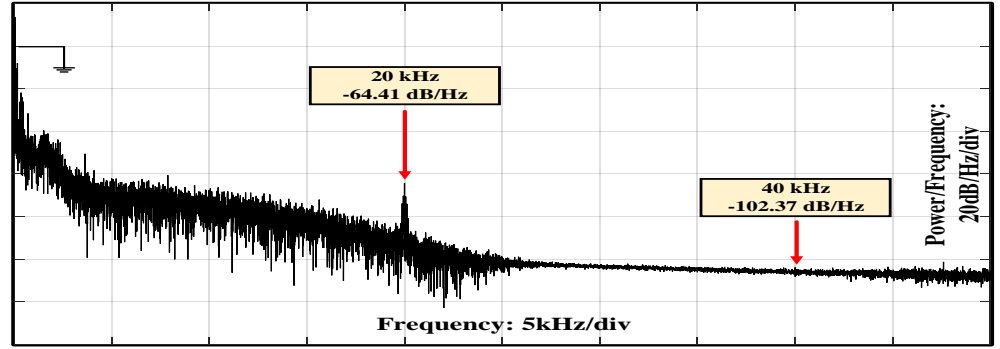

(b)

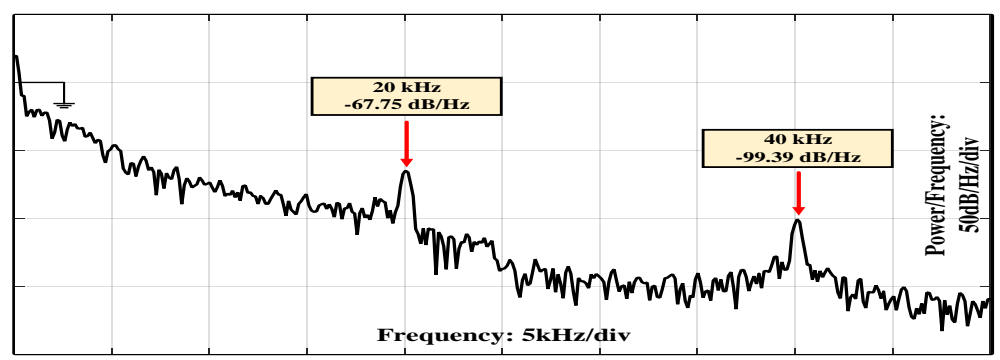

(c)

Figure 6.11: $L L C L$ filter. (a) Grid voltage and grid-side current waveforms. (b) Power density spectrum of grid-side current (Experimental setup). (c) Power density spectrum of grid-side current (Simulation)

and Fig. 6.12(b) illustrate that the amplitude of the dominant harmonic current at $20 \mathrm{kHz}$ is reduced by $8.80 \mathrm{~dB} / \mathrm{HZ}$ from case I to case II, but at $40 \mathrm{kHz}$ it increased from -102.37 $\mathrm{dB} / \mathrm{Hz}$ (case I) to $-97.01 \mathrm{~dB} / \mathrm{Hz}$ (case II). Therefore, the most dominant current harmonics are diminished even more than the previous design. However, a small increase occurred at the double of the switching frequency, but as the power density is too small (-97.01 $d B / H z)$, it can be neglected. Thus, the size of the filter is reduced, and as a result, the total loss is decreased. In addition, the performance of the filter is improved. Figs. 6.11 (c) and 6.12 (c) show the simulation results of the power density spectrum of the grid-side 


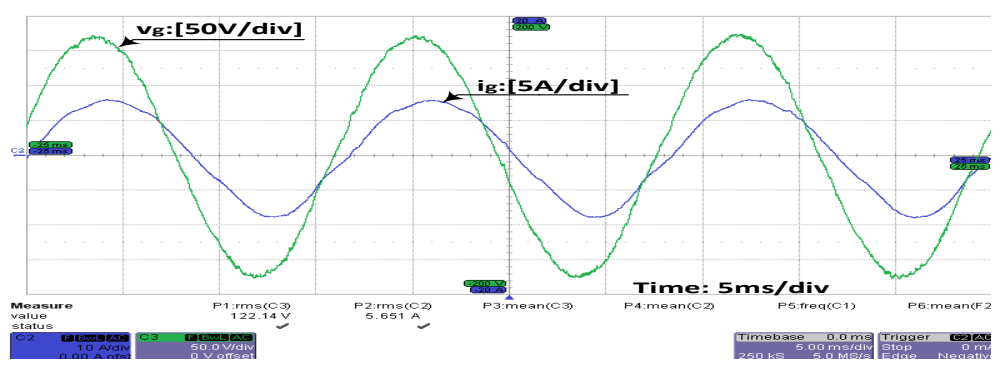

(a)

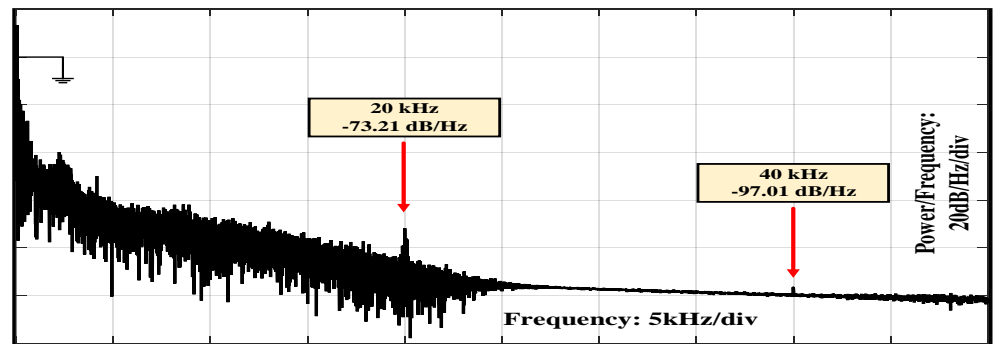

(b)

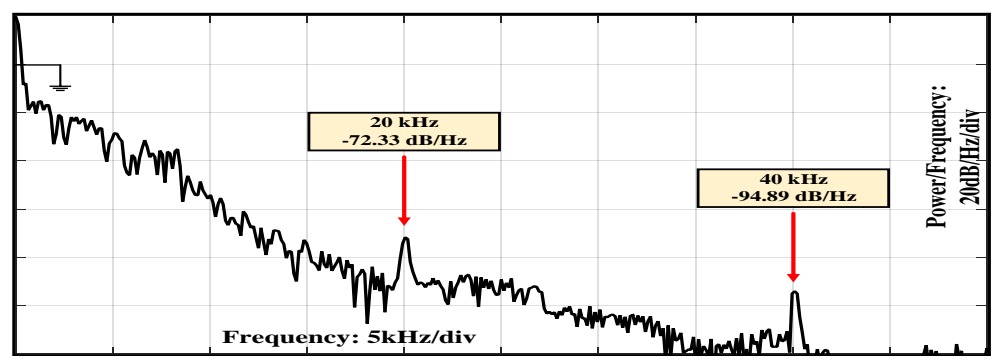

(c)

Figure 6.12: $L(L C L)_{2}$ filter. (a) Grid voltage and grid-side current waveforms. (b) Power density spectrum of grid-side current (Experimental setup). (c) Power density spectrum of grid-side current (Simulation).

current. As it can be seen, the experimental results are in accordance with the simulation graphs.

The currents flowing through the resonant branches for Case I and Case II are shown in Figs. 6.13 to 6.15 while the grid current is $5.8 \mathrm{~A}$ and the voltage is $120 \mathrm{~V}$. The magnitude of the currents in $L_{f 1} C_{f 1}$ and $L_{f 2} C_{f 2}$ traps are almost half of $L_{f} C_{f}$ trap and that is because the impedance of both traps of $L(L C L)_{2}$ filter is $2.652 k \Omega$ (at $60 \mathrm{~Hz}$ ), but the $L L C L$ filter has an impedance of $1.326 \mathrm{k} \Omega$. Also, it can be seen that the power density of current harmonics at the switching frequency and multiples of the switching frequency 


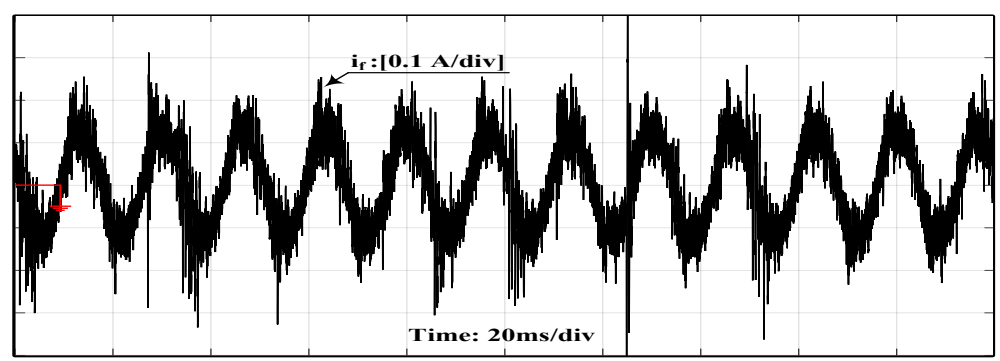

(a)

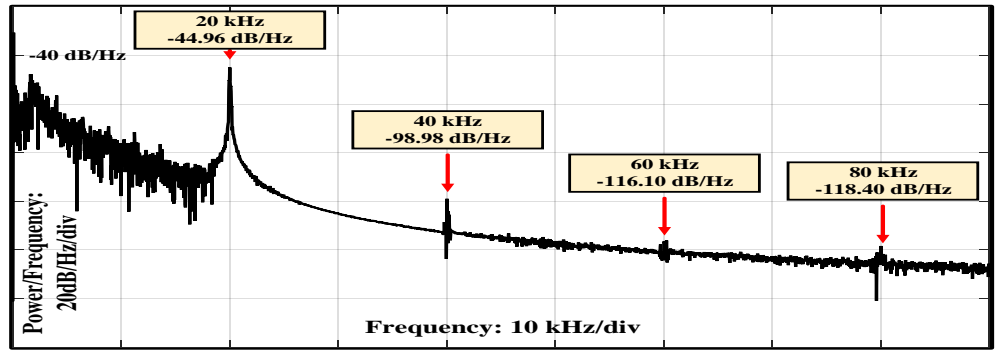

(b)

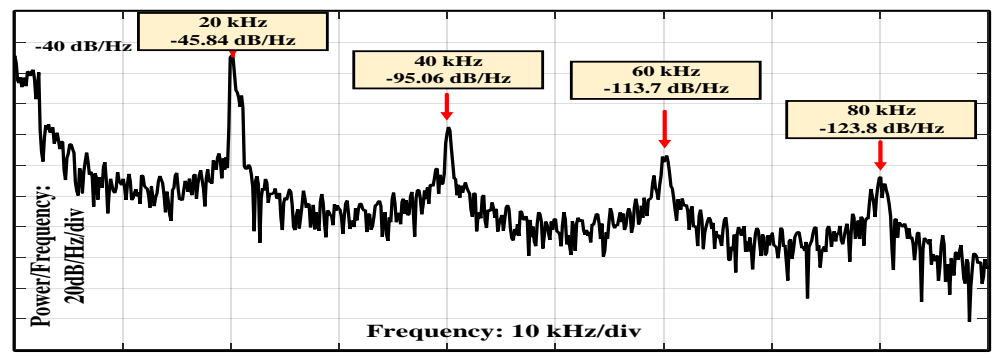

(c)

Figure 6.13: (a) $L_{f} C_{f}$ current of $L L C L$ filter . (b) Power spectral density of $L_{f} C_{f}$ current in $L L C L$ filter (Experimental setup). (b) Power spectral density of $L_{f} C_{f}$ current in $L L C L$ filter (Simulation).

are almost the same in the $L_{f} C_{f}$ branch of the $L L C L$ and $L_{f 1} C_{f 1}$ branch of the $L(L C L)_{2}$ filter. In addition to that more attenuation occurs at $L_{f 2} C_{f 2}$. Then, in Figs. 6.13(c) - 6.15 (c) simulation results are depicted which are quite close to the hardware experimental results.

The measured total harmonic distortion (THD)\% of $i_{g}$ in Cases I, and II are $3.72 \%$ and $2.94 \%$ which shows the effectiveness of the designed filter in improving the THD of the grid current.

Fig. 6.16 shows the bode plots of the $i_{g}(s) / v_{i}(s)$ transfer function for different values 


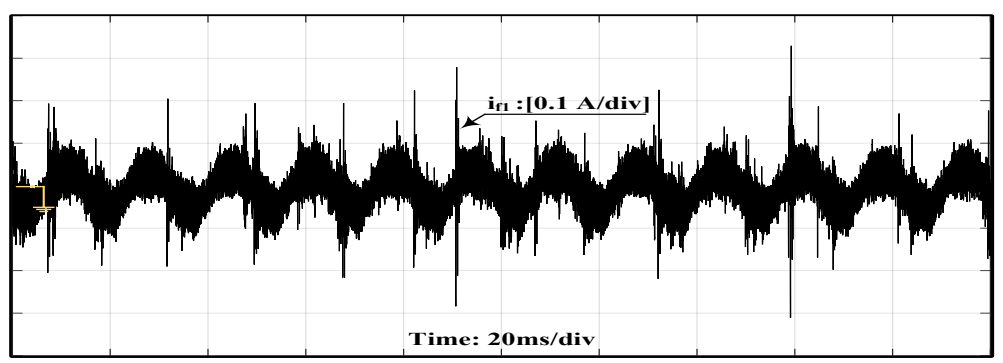

(a)

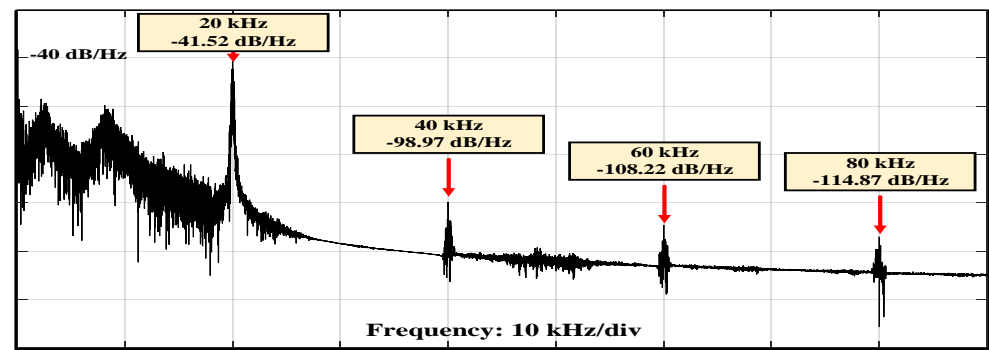

(b)

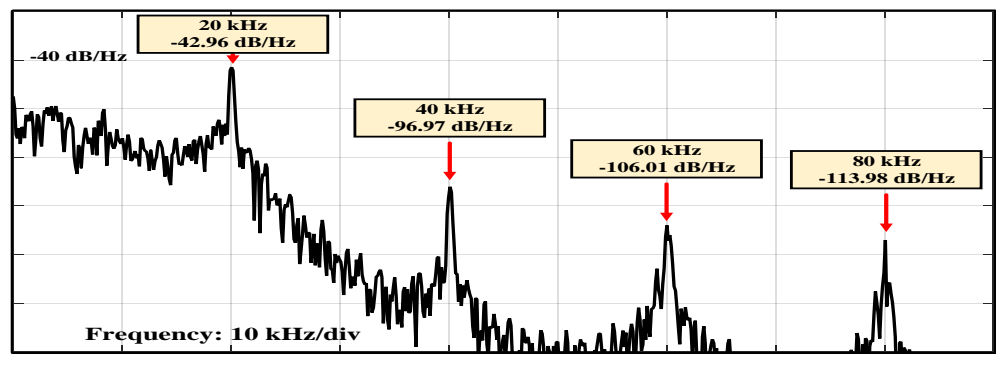

(c)

Figure 6.14: (a) Current of switching frequency trap $L_{f 1} C_{f 1}$ of $L(L C L)_{2}$ filter. (b) Power spectral density of $L_{f 1} C_{f 1}$ current in $L(L C L)_{2}$ filter (Experimental setup). (c) Power spectral density of $L_{f 1} C_{f 1}$ current in $L(L C L)_{2}$ filter (Simulation).

of the series and the resonant branch inductances. Obviously, when the values of $L_{12}, L_{22}$ and $L_{32}$ are in the range of parameter values generated by the proposed procedure, the resonant frequency variation meets the design criteria. Therefore, the system is still able to maintain stability. Furthermore, to guarantee that all current harmonic $\left(\geq 35^{\text {th }}\right)$ is less than $0.3 \%$ of the fundamental current, while it is assumed that the parameter variation of inductances of resonant branches are in a range of $\pm 20 \%$, it is essential to investigate the ripple current harmonics both at $f_{s}$ and at $2 f_{s}$ while these inductors change $\pm 20 \%$. The harmonics of current around the switching frequency with $L_{f 1} \pm 20 \%$ is $0.21 \%$ and the 


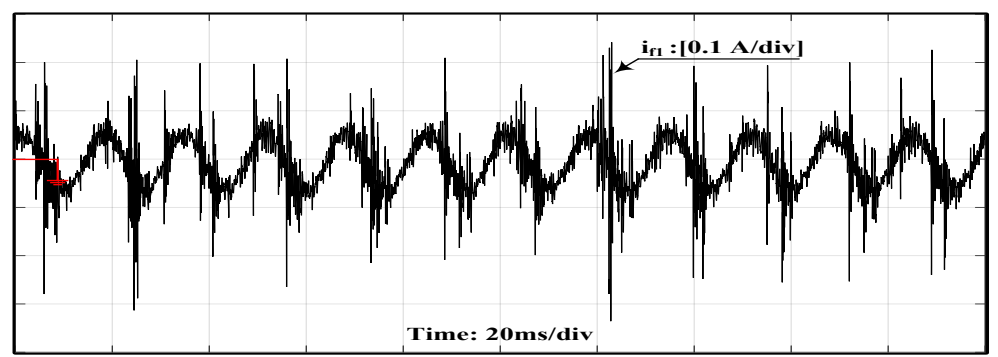

(a)

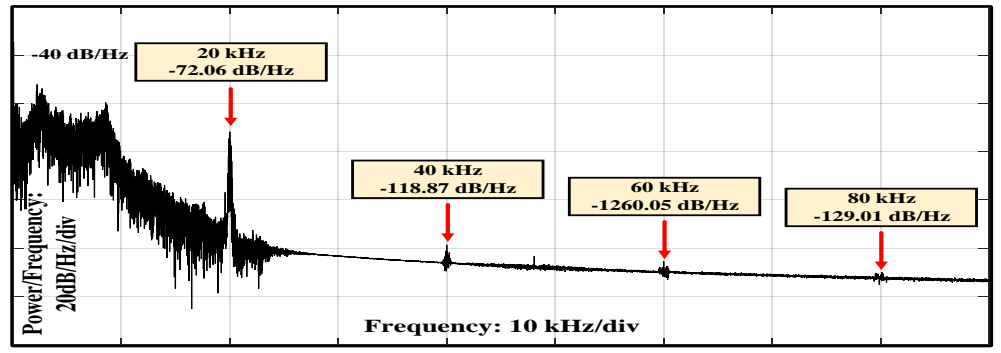

(b)

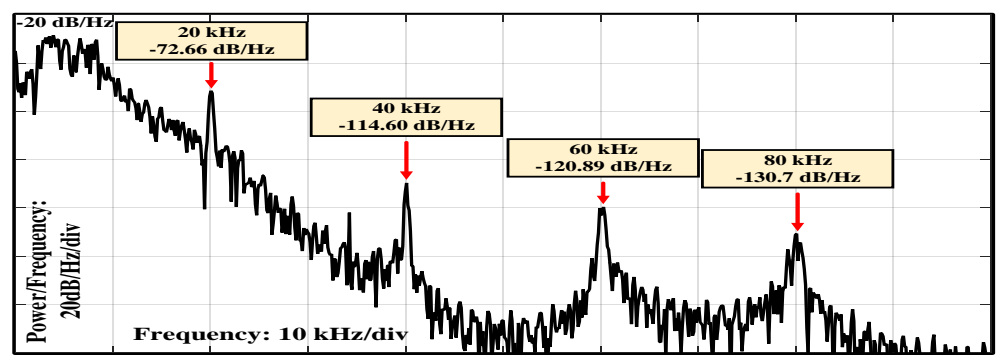

(c)

Figure 6.15: (a) Current of the double of switching frequency trap $L_{f 2} C_{f 2}$ of $L(L C L)_{2}$ filter . (b) Power spectral density of $L_{f 2} C_{f 2}$ current in $L(L C L)_{2}$ filter (Experimental setup). (c) Power spectral density of $L_{f 2} C_{f 2}$ current in $L(L C L)_{2}$ filter (Simulation).

current harmonics around double the switching frequency with $L_{f 2} \pm 20 \%$ is $0.017 \%$.

At last, the response of the developed filter is compared with that of an $L L C L$-based inverter in terms of voltage regulation. In order to do that, the simulations were run at a sampling time of $2 \mu \mathrm{s}$ and the values of Table 6.3. These values were designed such that the performance of the $L(L C L)_{2}$ filter is comparable to that of the $L L C L$ in terms of steady state performance and the dynamic response. Fig. 6.17 shows both cases when a $0.5 p u$ load is switched in at the point of grid connection on $t=0.15 \mathrm{~s}$. It can be seen that the inverter maintains a constant dc-link voltage before and after the change. Results show 


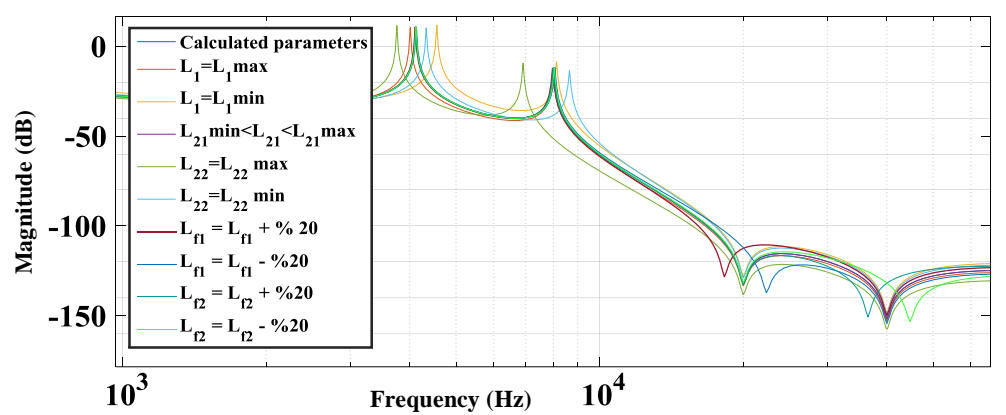

Figure 6.16: Bode plots of transfer function $i_{g}(s) / v_{i}(s)$ under different filter inductances.

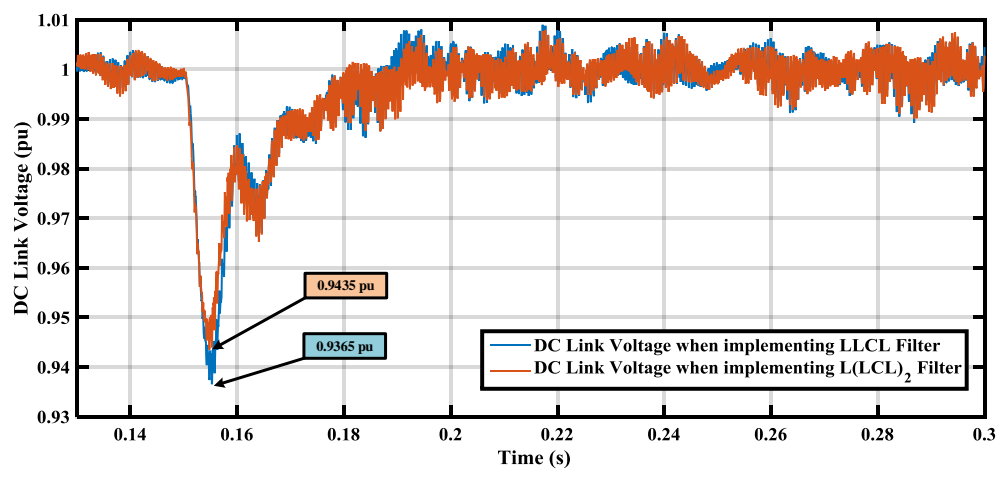

Figure 6.17: DC Link voltage when a 0.5 pu load is switched-in on $\mathrm{t}=0.15$.

that, replacing the $L L C L$ filter with an $L(L C L)_{2}$ filter improves the dynamic performance of the inverter mainly due to the reduction of dc-link voltage fluctuations.

The experimental setup of the $L(L C L)_{2}$-filter-based inverter system is shown in Fig. 6.18. For building the inductances of $L L C L$ and $L(L C L)_{2}$ filters Ferrite cores with N87 material and epoxy coating is used.

\subsection{Analysis and Discussion}

From the simulation and experimental results, the following can be seen.

1. In both cases, dominating harmonic current meets the recommendation of IEEE 519-2014 in the experiment, however, the $20 \mathrm{kHz}$ current harmonic reduced and the 


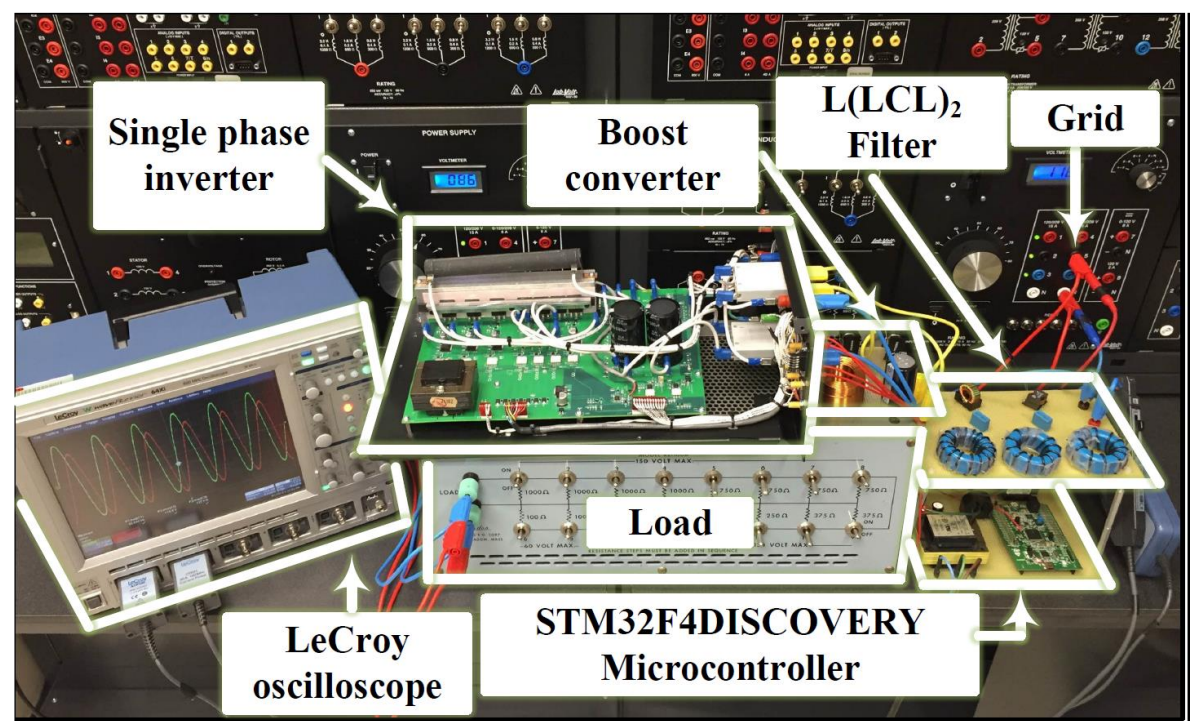

Figure 6.18: Experimental Setup

$40 \mathrm{kHz}$ current harmonic has the same value.

2. The value of the grid-side inductor is reduced in cases II, so the voltage drop during the operation and thereby the dc link voltage are the same.

3. The value of the total inductor of $L(L C L)_{2}$ filter is reduced by a factor of $22.22 \%$, compared to that of the $L L C L$ filter.

4. The reactive power in the newly designed filter is the same as the traditional $L L C L$ filter $C_{f 1}+C_{f 2}=C_{f}$.

5. In general, the experimental results are in acceptable agreement with the theoretical study, particularly with regard to the harmonic current attenuation around the switching frequency and the double of the switching frequency. 


\subsubsection{Power Loss Analysis of the $L(L C L)_{2}$ Filter components}

\subsubsection{High-frequency Damping Loss}

Ignoring the grid voltage harmonics and the dead-time effect, the most dominating highorder current harmonics contain the switching frequency and the double of the switching frequency. The damping loss in the high-frequency can be obtained as follows [22]

$$
P_{d}(\text { ripple })=\frac{1}{2}\left|I_{A M}\left(n, k_{1}\right)\right|^{2} \times R_{f 1}+\left|I_{A M}\left(n, k_{2}\right)\right|^{2} \times R_{f 2}
$$

Where $n= \pm 1, \pm 3, \pm 5, k_{1}=1$ and $k_{2}=2$.

\subsubsection{Power Losses in Inductors}

The losses of the inductor can be separated into core loss $\left(P_{\text {core }}\right)$ and copper loss $\left(P_{\text {copper }}\right)$. The core loss includes the hysteresis loss, the eddy current loss and the residual loss in the magnetic material. The copper loss includes the loss produced by the current at fundamental frequency and ripple current at the switching frequency in the windings. The power loss of inductor differs with the type of winding, type of the core material, and the switching frequency.

2-1) Copper losses calculation: In general, the proximity and skin effects have contribution in the copper loss of the windings. In the case of the grid-side inductor $L_{32}$, the high- frequency ripple current is adequately mitigated by the filter to meet the requirements, thus, the power loss will only be as a result of the fundamental frequency current. The copper loss of the series inductors excluding inverter-side inductors can be calculated as

$$
P_{\text {copper }}=I_{R M S}^{2} R_{d c}+{I_{a c}}^{2} R_{a c}
$$

where $I_{R M S}$ is the RMS value of the current in fundamental frequency and $I_{a c}$ is the RMS value of the ac-ripple current component. In equation (6.29), $R_{d c}$ and $R_{a c}$ are dc- 
resistance and the ac-resistance of the series inductances except the inverter side inductance. For the solid conductor copper with the American wire gauge (AWG) of 22, the maximum frequency for $100 \%$ skin depth is $42 \mathrm{kHz}$. Consequently, it can be assumed that $R_{a c}=R_{d c}$ in this study. The inverter-side inductor conduction loss is given by

$$
P_{L G S}=I_{R M S_{1}}^{2} R_{d c_{1}}
$$

where $R_{d c_{1}}$ is the resistance of the inverter-side inductor. and $I_{R M S_{1}}$ is the RMS inverterside inductor current, given by

$$
I_{R M S_{1}}{ }^{2}=I_{R M S}^{2}+\frac{\triangle i_{p p}{ }^{2}}{12}
$$

2-2) Core loss calculation: The core loss can be found with the satisfactory accuracy using the Steinmetz equations supplied by the manufacturer's datasheet, using the following equation

$$
P_{\text {core }}=k\left(f^{\alpha} B^{\beta} V_{e}\right)
$$

where $k, \alpha$, and $\beta$ are parameters of material typically indicated by curve fitting; $\mathrm{B}$ is the flux density, in Tesla and $V_{e}$ is the magnetic core volume, in $\mathrm{cm}^{3}$. The equation only works with a sinusoidal frequency and does not consider factors such as dc-offset.

Table 6.4: Parameters Which Are Used for Theoretical Converter Loss Calculation.

\begin{tabular}{lll}
\hline Parameter & Description & $\begin{array}{l}\text { Typical } \\
\text { Value }\end{array}$ \\
\hline$I_{R M S_{1}} L L C L$ & RMS value of the current & $5.8333 \mathrm{~A}$ \\
$I_{R M S_{1}} L(L C L)_{2}$ & RMS value of the current & $5.8336 \mathrm{~A}$ \\
$R_{F}$ & $\begin{array}{l}\text { Equivalent ON-state resis- } \\
\text { tance }\end{array}$ & $0.1 \Omega$ \\
$C_{\text {oss }}$ & $\begin{array}{l}\text { Output capacitance } \\
E_{\text {on }}+E_{\text {off }}\end{array}$ & $\begin{array}{l}\text { Switching volt-ampere } \\
\text { crossover energy losses }\end{array}$ \\
& & $0.5 \mathrm{~mJ}$ \\
\hline
\end{tabular}




\subsubsection{Inverter Loss Analysis}

The power loss of the inverter can be calculated by evaluating the switching and the conduction losses of the power switches. The loss of each power switch can be written as follow [231]

$$
\begin{aligned}
P_{S x}= & P_{S c o n}+P_{S s w}=\left[R_{F} I_{R M S_{1}}^{2}\right] \\
& +\left[\left(E_{\text {on }}+E_{\text {off }}\right)+\frac{1}{2} C_{\text {oss }} V_{\text {in }}^{2}\right] f_{s}
\end{aligned}
$$

where $P_{S c o n d}$ and $P_{S s w}$ are the conduction and switching losses of the switch $S_{x}$ respectively, $R_{F}$ is the equivalent resistance of the switch during on state, $E_{\text {on }}$ and $E_{\text {off }}$ are volt-ampere crossover energy losses during the switch turn-on and turn-off transitions; respectively, $C_{\text {oss }}$ is the output capacitance of the switch, and $V_{i n}$ is the input voltage. Typical values which are used in the inverter, are presented in Table 6.4. Using equation (6.33) and Table 6.4 the efficiency of the inverter connected to a conventional LLCL filter is $95.2359 \%$ and the efficiency of the inverter connected to the proposed $L(L C L)_{2}$ is 95.2357\%. Therefore, it can be concluded that the increased current ripple which is due to the use of $L(L C L)_{2}$ filter, has a negligible effect on the efficiency of the inverter.

The power loss values of the $L(L C L)_{2}$ filter and the $L L C L$ filter are presented in Table 6.5. It can be seen that besides high- frequency damping loss, other losses are less in the $L(L C L)_{2}$ filter. However, the total power loss is decreased by $3.12 \%$, compared to that of the $L L C L$ filter. 
Table 6.5: Power Losses of the $L(L C L)_{2}$ Filter and The $L L C L$ Filter.

\begin{tabular}{lll}
\hline & LLCL & L $(\text { LCL })_{2}$ \\
& Filter & Filter \\
\hline $\mathbf{P}_{d}$ & $0.0064 \mathrm{w}$ & $0.0345 \mathrm{w}$ \\
$\mathbf{P}_{\text {copper }}$ & $1.1840 \mathrm{w}$ & $1.1092 \mathrm{w}$ \\
$\mathbf{P}_{L G S}$ & $2.2865 \mathrm{w}$ & $1.6494 \mathrm{w}$ \\
$\mathbf{P}_{\text {core }}$ & $1.8238 \mathrm{w}$ & $1.3015 \mathrm{w}$ \\
$\mathbf{P}_{S x}$ & $33.3487 \mathrm{w}$ & $33.3501 \mathrm{w}$ \\
\hline Total & $\mathbf{3 8 . 6 4 9 4} \mathbf{w}$ & $\mathbf{3 7 . 4 4 4 7 ~ w}$ \\
Loss & &
\end{tabular}

\subsubsection{Small-signal model of the dc-link dynamics considering the in- stantaneous power for bi-directional ac/dc power flow applica- tions}

If the $L(L C L)_{2}$ filter is employed in bi-directional ac/dc power flow applications, the converter should be able to regulate dc voltage, and the instantaneous power stored in the ac side filter affects the dc side dynamics. In this section the small-signal model of the dc-link dynamics considering the instantaneous power is studied.

In high-order ac-side filters applications - e.g., $L C L, L L C L$ or $L(L C L)_{2}$ filter due to the frequency-scale separation of the high order filter dynamics and dc-link voltage dynamics, the filter has a negligible effect on the dc-link voltage dynamics. The fundamental frequency is normally higher than the bandwidth of the dc-link voltage control loop (between 20 to $50 \mathrm{~Hz})$. The high order ac-side filter acts mainly as an $\mathrm{L}$ filter $\left(L_{12}+L_{22}+L_{32}\right)$ [232].

The dc-link dynamics model can be obtained when the total instantaneous power in the storage devicesi.e., $L_{\mathrm{DC}-\mathrm{eq}}$ and $C_{\mathrm{eq}}$ is considered. The calculations on this section are based on [233] and [234]. $C_{\mathrm{eq}}$ and $L_{\mathrm{DC}-\text { eq }}$ are the equivalent capacitance and inductance reflected to the dc side VSC, respectively. The power balance across the dc-link capacitor 
can be given by

$$
\begin{gathered}
P_{\mathrm{ext}}-P_{\mathrm{loss}}-\frac{V_{\mathrm{DC}}^{2}}{R_{L}}-D\left(0.5 L_{\mathrm{DC}-\mathrm{eq}} I_{\mathrm{DC}}^{2}\right) \\
-D\left(0.5 C_{\mathrm{eq}} V_{\mathrm{DC}}^{2}\right)=P_{\mathrm{DC}}=P_{t}
\end{gathered}
$$

where $I_{\mathrm{DC}}$ is the current of the dc inductor; $P_{\mathrm{ext}}$ is the external injected power to the dc side; $P_{\text {loss }}$ is the the losses in the converter; $R_{L}$ is the VSC reflected dc side equivalent static resistance; $P_{D C}$ is the net power as shown in Fig 6.3, that is equivalent to the VSC ac-side power $P_{t}$; and the time-derivative operator is represented by $\mathrm{D}$ - i.e., $D x=$ $d(x) / d t$. Consider that $P t$ does not have the same value of the grid injected power $\left(P_{s}\right)$, because of the ac-side filter.

By using similar calculation in [233] and letting $x_{1}=V_{\mathrm{DC}}$ and $x_{2}=D V_{\mathrm{DC}}$; the power balance equation can be calculated as follows

$$
\begin{aligned}
\tilde{P}_{\text {ext }} & -\tilde{P}_{\text {loss }}-\tilde{P}_{\mathrm{DC}}-L_{\mathrm{DC}-\mathrm{eq}} C_{\mathrm{eq}} \frac{P_{\mathrm{DC}-0}}{V_{\mathrm{DC}-\text { nominal }}} \tilde{D} x_{2} \\
& -L_{\mathrm{DC}-\mathrm{eq}} \frac{P_{\mathrm{DC}-0}}{V_{\mathrm{DC}-\text { nominal }}^{2}} \tilde{D} P_{\mathrm{DC}}+L_{\mathrm{DC}-\mathrm{eq}} \frac{P_{\mathrm{DC}-0}^{2}}{V_{\mathrm{DC}-\text { nominal }}^{3}} \tilde{x}_{2} \\
& -C_{\mathrm{eq}} V_{\mathrm{DC}-\text { nominal }} \tilde{x}_{2}-\frac{2 V_{\mathrm{DC}-\text { nominal }}}{R_{p}} \tilde{x}_{1}=0 .
\end{aligned}
$$

where $\sim$ indicates the perturbed signal around the equilibrium point (E.P.) of each variable. Moreover, the equilibrium point (E.P.) can be expressed by (6.36) taken into account that all variables are static at the equilibrium situation

$$
\begin{aligned}
(\text { E.P. }) & =\left(x_{1-0}, x_{2-0}, D x_{2-0} P_{\mathrm{DC}-0}, D P_{\mathrm{DC}-0}, P_{\text {ext }-0}, P_{\text {loss }-0}\right) \\
& =\left(V_{\mathrm{DC}-\text { nominal }}, 0,0, P_{\mathrm{DC}-0}, 0, P_{\text {ext }-0}, P_{\text {loss }-0}\right)
\end{aligned}
$$

According to equation (6.35), with the purpose of reaching a linear time-invariant (LTI) model that describe the connection between the output $V_{D C}$ and the control input $I_{g}$, a formula which connects $P_{D C}$ to the control input $I_{g}$ have to be calculated. 
Therefore, studying the total power balance on the ac side is necessary for analyzing the instantaneous power of the ac-side filter. This power balance is defined as follows

$$
P_{\mathrm{DC}}=P_{t}=P_{\text {loss } \_}+P_{\text {filter } \_L}+P_{s}
$$

where $P_{\text {loss }} R$ is the total instantaneous power loss in the equivalent resistance of the inductor $L_{12}, L_{22}$ and $L_{32}, P_{\text {loss }_{L}}$ is the total instantaneous power of the ac-side inductor $\left(L_{12}+L_{22}+L_{32}\right)$, and $P_{s}$ is the total absorbed/injected instantaneous power at the point of common coupling (PCC) for stabilizing the dc-link voltage. The total absorbed instantaneous power by a single phase network can be shown by

$$
P_{\text {single phase network }}=\operatorname{Re}\left\{\vec{v}(t) \vec{i}^{*}(t)\right\}
$$

The total stored energy in the filter inductors is expressed by

$$
W_{L}=0.5\left(L_{12}+L_{22}+L_{32}\right)\left(i_{g}^{2}\right)
$$

where $i_{g}$ is the current injected into the inductors $L_{12}, L_{22}$ and $L_{32}$. As a result, (6.40) is the expression of the instantaneous power of the filter series inductors.

$$
P_{\text {filter } \_L}=\left(L_{12}+L_{22}+L_{32}\right) i_{g} D i_{g}
$$

and $P_{s}$ can be calculated by:

$$
P_{s}=I_{g} V_{g_{s}}
$$

where $V_{g_{s}}$ is the voltage space vector at the PCC. Using (6.37) - (6.41), the relationship between $P_{D C}, I_{g}$ can be shown by

$$
\begin{array}{r}
P_{\mathrm{DC}}-\left(L_{12}+L_{22}+L_{32}\right) i_{g} D i_{g}-R\left(I_{g}\right)^{2}-I_{g} V_{g_{s}} \\
=G\left(I_{g}, D I_{g}\right)=0 .
\end{array}
$$


Equation (6.42) is nonlinear, therefore the Taylor series expansion around one E.P. is applied on the dynamic function $G$ as

$$
\begin{aligned}
G\left(I_{g}, D I_{g}\right)= & \left.G\right|_{(\text {E.P. })}+\left.\frac{\partial G}{\partial I_{g}}\right|_{(\text {E.P. })} \tilde{I}_{g} \\
& +\left.\frac{\partial G}{\partial D I_{g}}\right|_{(\text {E.P. })} \tilde{D} I_{g}+\{\text { H.O.T. }\}
\end{aligned}
$$

The E.P. of (6.43) can be shown by

$$
(\text { E.P. })=\left(I_{g-0}, D I_{g-0}\right)=\left(I_{g-0}, 0\right)
$$

After mathematical manipulation, (6.45) can be reached.

$$
\tilde{P}_{\mathrm{DC}}=L I_{g-0} \tilde{D} I_{g}+R I_{g-0} \tilde{I}_{g}+V_{g_{s}} \tilde{I}_{g}
$$

In addition to $(6.45), D \tilde{P}_{\mathrm{DC}}$ is also required to find the explicit transfer function between $I_{g}$ and $V_{D C}$ using (6.35). Using (6.45), D $\tilde{P}_{\mathrm{DC}}$ and its E.P. can be given by

$$
\begin{aligned}
D P_{\mathrm{DC}} & -\left(L_{12}+L_{22}+L_{32}\right)\left(\left(D I_{g}\right)^{2}+I_{g} D^{2} I_{g}\right) \\
& -2 R I_{g} D I_{g}-V_{g_{s}} D I_{g} \\
& =H\left(I_{g}, D I_{g}, D^{2} I_{g}\right)=0 \\
(\text { E.P. }) & =\left(I_{g-0}, D I_{g-0}, D^{2} I_{g-0}\right) \\
& =\left(I_{g-0}, 0,0\right) .
\end{aligned}
$$

By implementing the Taylor series expansion on the function $\mathrm{H}$ in $(6.46), D \tilde{P}_{\mathrm{DC}}$ can be calculated by

$$
\begin{aligned}
D \tilde{P}_{\mathrm{DC}} & =\left(L_{12}+L_{22}+L_{32}\right) I_{g-0} D^{2} \tilde{I}_{g}+R I_{g-0} D \tilde{I}_{g} \\
& +V_{g_{s}} D \tilde{I}_{g}
\end{aligned}
$$


By combining the equations (6.35), (6.45), and (6.48), the relationship between $I_{g}$ and $V_{D C}$ is reached as follows

$$
\begin{aligned}
\tilde{V}_{\mathrm{DC}} & =\frac{\tilde{P}_{\text {ext }}-\tilde{P}_{\text {loss }}}{A s^{2}+B s+E}-\frac{a s^{2}+b s+e}{A s^{2}+B s+E} \tilde{I}_{g} \\
a & \triangleq-L_{\mathrm{DC}-\mathrm{eq}}\left(L_{12}+L_{22}+L_{32}\right) \frac{P_{\mathrm{DC}-0} I_{g-0}}{V_{\mathrm{DC}-\text { nominal }}^{2}} \\
b & \triangleq-\left(L_{12}+L_{22}+L_{32}\right) I_{g-0} \\
& -L_{\mathrm{DC}-\mathrm{eq}} \frac{P_{\mathrm{DC}-0}}{V_{\mathrm{DC}-\text { nominal }}^{2}}\left(R I_{g-0}+V_{g_{s}}\right) \\
e & \triangleq-R I_{g-0}-V_{g_{s}} \\
A & \triangleq L_{\mathrm{DC}-\mathrm{eq}} C_{\mathrm{eq}} \frac{P_{\mathrm{DC}-0}}{V_{\mathrm{DC}-\text { nominal }}} \\
B & \triangleq\left(C_{\mathrm{eq}} V_{\mathrm{DC}-\text { nominal }}-L_{\mathrm{DC}-\mathrm{eq}} \frac{P_{\mathrm{DC}-0}^{2}}{V_{\mathrm{DC}-\mathrm{nominal}}^{3}}\right) \\
E & \triangleq\left(\frac{2}{R_{L}} V_{\mathrm{DC}-\text { nominal }}\right)
\end{aligned}
$$

In many different references, e.g. [235,236], the effect of the dc filter is not taken into the consideration, while the ac filter instantaneous power is reflected into the dynamic analysis of the dc-link voltage. In that situation, $L_{\mathrm{DC}-\mathrm{eq}}$ is set to zero which results in (6.50) becoming a special case of (6.49). The values of a, b, e, A, B, and E are given in 
(6.50) for $L_{\mathrm{DC}-\mathrm{eq}}=0$.

$$
\begin{aligned}
a= & 0 \\
b= & -\left(L_{12}+L_{22}+L_{32}\right) I_{g-0} \\
e \triangleq & -R I_{g-0}-V_{g_{s}} \\
A= & 0 \text { then } \\
\tilde{V}_{\mathrm{DC}}= & \frac{-\left(L_{12}+L_{22}+L_{32}\right) I_{g-0} s-V_{g_{s}}-R I_{g-0}}{C_{\mathrm{eq}} V_{\mathrm{DC}-\text { nominal }} s+\frac{2}{R_{L}} V_{\mathrm{DC}-\text { nominal }}} \\
& - \text { Disturbance Signals. }= \\
B= & \frac{2}{R_{L}} V_{\mathrm{DC}-\text { nominal }} V_{\mathrm{DC}-\text { nominal }} \\
E &
\end{aligned}
$$

In (6.50), in both rectification and inversion modes, the magnitude of the linearized plant is the same. On the other hand, in the rectification mode the phase is dramatically reduced at the same power, due to the right-hand-plane (RHP) zero. If the time constant of the current controller is equal to zero, in order to have a stable transfer function $\mathrm{B}$ and $\mathrm{E}$ in (6.50) should be positive. As $C_{\mathrm{eq}}, V_{\mathrm{DC}-\text { nominal }}$ and $R_{L}$ are always positive numbers, (6.50) is always stable in bidirectional ac/dc power flow application.

\subsection{Summary}

In chapter 6 , the principles of the conventional $L L C L$ filter and parameter design of the $L(L C L)_{2}$ filters has been proposed. Since grid-side inductance $\left(L_{21}\right)$ of the $L L C L$ filter is mainly decided by the harmonic currents around double the switching frequency instead of those around the switching frequency, it has been replaced by a small trap at double the switching frequency. Compared to the $L L C L$ filter, the replacement results in reduction of the total inductance size, and hence, the total loss of the filter. The inverter-side inductance

is divided into three parts to place resonant branches in between them. Therefore, the 
$L(L C L)_{2}$ filter has lower loss and better performance at high-order harmonics attenuation. In the proposed design, the maximum power factor variation remained unchanged and the current THD has improved by $7.77 \%$.

A $700 \mathrm{~W}$ single-phase grid-tied inverter is designed to compare the characteristics of the conventional $L L C L$ filter and the suggested $L(L C L)_{2}$ filter through experimental results. In addition, the stability of the closed-loop system has been analyzed. The results validate that total loss and the value of the inductors of the $L(L C L)_{2}$ filter, reduced by a factor of $3.12 \%$ and $22.22 \%$; respectively, compared to that of the $L L C L$ filter. 


\section{CHAPTER 7}

\section{A COMBINATORIAL ADVANCED SOLUTION FOR PV INTEGRATION TO ADDRESS INTERMITTENCY AND PROVIDE INERTIAL RESPONSE}

\subsection{Overview}

Operation of a photovoltaic (PV) generating system under intermittent solar radiation is a challenging task. Furthermore, with high-penetration levels of photovoltaic energy sources being integrated into the current electric power grid, the performance of the conventional synchronous generators is being changed and grid inertial response is deteriorating. From an engineering standpoint, additional technical measures by the grid operators will be done to confirm the increasingly strict supply criteria in the new inverter dominated grid conditions. This chapter proposes a combined virtual inertia emulator (VIE) and a hybrid battery-supercapacitor-based energy storage system . VIE provides a method which is based on power devices (like inverters), which makes a compatible weak grid for integration of renewable generators of electricity. This method makes the power inverters behave more similar to synchronous machines. Consequently, the synchronous machine properties, which have described the attributes of the grid up to now, will remain active, although after integration of renewable energies. Examples of some of these properties are grid and generator interactions in the function of a remote power dispatch, transients reactions, and the electrical outcomes of a rotating bulk mass.

The hybrid energy storage system (HESS) is implemented to smooth the short-term power fluctuations and main reserve that allows renewable electricity generators such as PV to be considered very closely like regular rotating power generators. The objective of utilizing the HESS is to add/subtract power to/from the PV output in order to smooth out the high frequency fluctuations of the PV power, which may occur due to shadows of passing cloud on the PV panels. A control system designed and challenged by providing 
a solution to reduce short-term PV output variability, stabilizing the DC link voltage and avoiding short term shocks to the battery in terms of capacity and ramp rate capability.

Not only could the suggested system overcome the slow response of battery system (including dynamics of battery, controller, and converter operation) by redirecting the power surges to the supercapacitor system, but also enhance the inertial response by emulating the kinetic inertia of synchronous generator.

Control systems for the VIE and battery-supercapacitor storage system are presented in this chapter. Correspondingly simulation results are discussed to validate the effectiveness of the proposed scheme. In this chapter, Matlab Simulink software has been used for developing control designs of VIE and Hybrid Energy Storage System (HESS). Through these studies, it will be demonstrated that the recommended method is capable of achieving voltage and frequency regulation and effective management of the hybrid storage system. Since the suggested technique focuses on short term fluctuations and includes no long-term power regulation, it needs no mass storage device. Thus the method is economical. The other concerns raised by renewables (e.g., forecast accuracy, low voltage ride-through, etc.) have not been addressed within this study. The following are some of the highlights of our proposed system:

\section{Highlights:}

These are the highlights of the proposed system: 1- There is no need to control the dc link separately. It will be controlled by HESS.2- Smoothing power and enhancing system inertial response at the same time. 3- The simulation is designed to be as close as possible to real system. 3-Fast voltage regulation. 4- Lowering charge/discharge current rates of battery. 5- Reducing Current stress levels on battery. 6- Improving life span of Battery.

To the best of our knowledge, we make the first attempt in approaching the mixedhybrid battery-supercapasitor energy storage system and virtual inertia emulator to mitigate the challenges of PV intermittency (in generation side) and stability (due to interrup- 
tions and initial current of big loads).

\subsection{Problem Statement}

As an environmental friendly and renewable energy source, solar generation has recently observed accelerated proliferation throughout the world [26]. One technical challenge of adding high levels of PV generation in the electric grid is the decline of inertial response which is a consequence of incompatibilities amongst the power demand and generation in the (micro) grid [237].

Structure of the grid is changing in way that the traditional one-way vertical configuration from power plants to consumers with very powerful, high capacity, but few generators (fig. 7.1, right) is increasingly transforming to formless, intensely meshed distribution system, with lots of grid connected renewable energies that have stochastic characteristics (fig. 7.1, left)[238]. The new structure necessitates the implementation of electronic based power conditioning devices in place of synchronous generators. Fig. 7.1 shows how the frequency stabilization increases with the number of synchronous machines.

Therefore, based on the regional distribution generations and the capacity of grid, there might be a line impedance increase and short circuit power reduction. This can promote or cause the mitigation and form of different disturbances. The conventional inverters and converters are currently utilized in integration of renewable generators, which their properties varies by the executed control architecture. Regularly controlled inverters for output voltage regulation by space-vector modulators are utilized to stabilize the grid locally and provide the reactive and active power requirement of the grid. The weaknesses of this functioning attitude is the great dissimilarities of grid performance when 


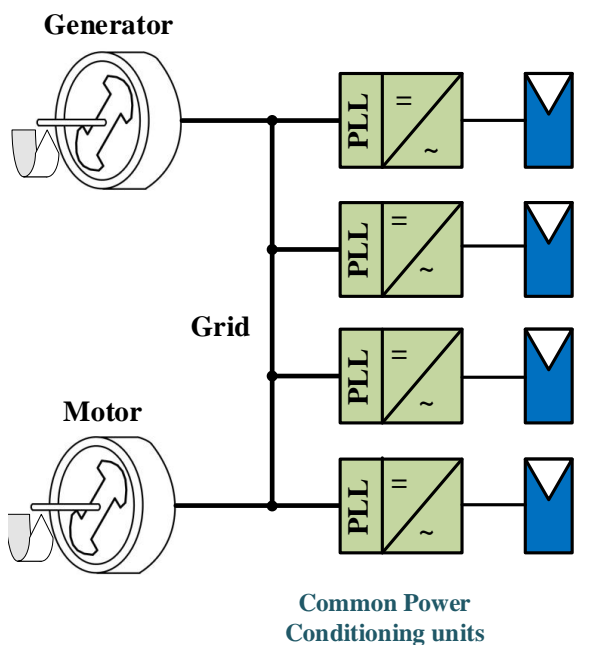

$$
\begin{gathered}
\frac{d}{d t}\left[\frac{f}{f_{0}}\right] \sim \frac{1}{t_{n}} \frac{\Delta P}{P_{\text {nom }}} \\
t_{n}=\sum m_{\text {gen }} \\
t_{n} \text { is the inertia of the grid }
\end{gathered}
$$
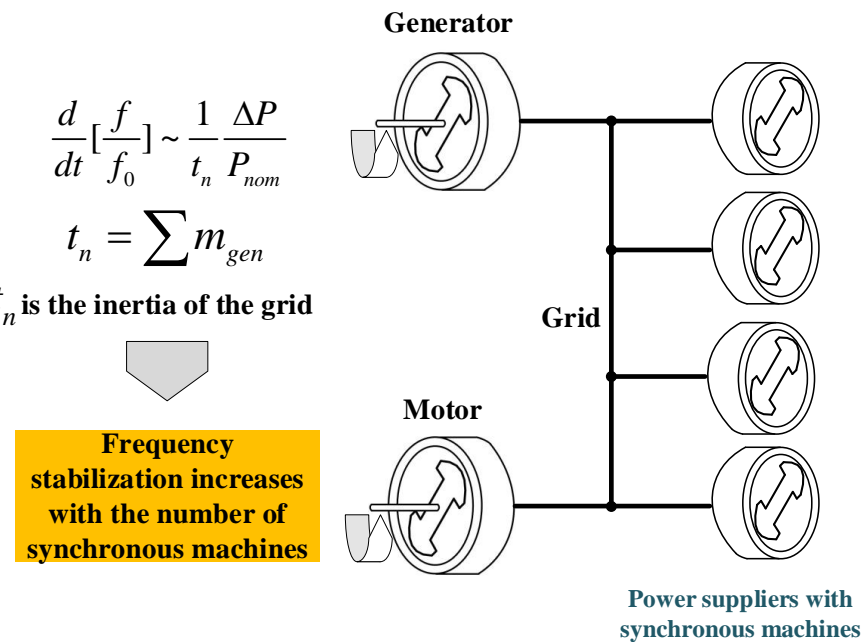

Figure 7.1: Future grid that will be dominated by power electronic inverters (left) and traditional grid dominated by synchronous machines (right)

grid connected inverters are exploited or the conventional synchronous machines are used for connecting old-fashioned prime movers to the grid [25].

Currently the dynamic and static properties of the electromechanical synchronous machines enforces the operating strategy and properties of electrical grids, repeatedly balancing the power deficit between different grid areas, and the dynamic behavior of the grid. However, this pattern is changing very fast to meet the new grid requirements.

Subject to increasing the instantaneous power or loads with large startup current, energy management and power control of a system with low rotational inertia is a vital concern. Such a high current in a short time not only requires greater rating of the power apparatuses, but also can possibly cause the system voltage and frequency to drop in the entire microgrid [239].

Therefore, it is essential to improve a method by which renewable generator inverters could be connected to any type the grid in such a way that the inverter-generator combination performs similarly to a synchronous generator. Additionally, a big change in load within a low inertia microgrid could cause a transient stability problem when it is 
islanded, and the same disturbance might pose a small-signal stability problem when it is grid connected [240]. In recent years, the concept of virtual synchronous machines has emerged as an effective method for adding virtual inertia to the power system through the control of power electronic converters [241].

Because of the stochastic nature of solar irradiation, the subsequent fluctuations in solar energy substantially handicap large-scale integration of PV into regional power grids. Subject to increasing the instantaneous power or loads with large startup current, energy management and power control of a system with low rotational inertia is a vital concern. Such a high current in a short time not only requires greater rating of the power apparatuses, but also can possibly cause the system voltage and frequency to drop in the entire microgrid [239]. What will be the effect of fluctuating real power output from renewable sources on the normal operation and power quality of the distribution system?

- Increased switching operations for line regulators, tap changers, switched-capacitors.

- Steady-state voltage regulation over the range of real power generation, especially on long feeders. Should PV generators be allowed/required to participate in voltage regulation automatically, or on the basis of reactive power dispatch or scheduling? If autonomous local automatic voltage control is allowed, can stable operation be expected when multiple PV generators are involved on the same feeder? Will fast automatic voltage controllers "fight" with slower line regulators?

- Flicker due to rapidly fluctuating voltage caused by sudden changes in real power generation.

- Transient voltage changes on sudden trip of PV generation system, especially if the system is actively participating in voltage regulation.

- Harmonics generated by the PV inverters, and possible resonant interactions of inverters with the distribution system. 
- Conductor and equipment loading due to new power flows resulting from the introduction of local power generation in the distribution system.

A possible solution for regulating the natural fluctuating output power of a PV plant is to integrate a hybrid energy storage system (HESS) that has both high energy density storage battery and high power density storage supercapacitor [7].

More than that, delivering high power in a short period of time is destructive to batteries, but it is the challenge that supercapacitor can best mitigate. In peak power situations, the supercapacitor is capable of delivering or receiving energy, therefore it can act as a load-flattening device for the battery. If this is done, the battery output power would become closer to the average load demand, hence decreasing its RMS and peak currents [23].

Finally, to the best of our knowledge, we make the first attempt in approaching the mixed-hybrid battery-supercapasitor energy storage system and virtual inertia emulator to mitigate the challenges of PV intermittency (in generation side) and stability (due to interruptions and initial current of big loads).

The chapter is organized as follows, in Section II: the description of the system is presented In Section III: the operation and modeling of the hybrid super capacitor/battery storage systems is explained. In Section IV: the control of the Virtual Inertia Emulator dynamic formulation of the synchronous generators are explained. In Section V: the simulation results are presented and discussed. Finally, in Section VI the conclusions that can be drawn out of this chapter is presented .

\subsection{Solution Approach}

The principle of VIE is based on merging the advantages of dynamic performance of inverter technology with the dynamic and static operational behavior of an electrome- 


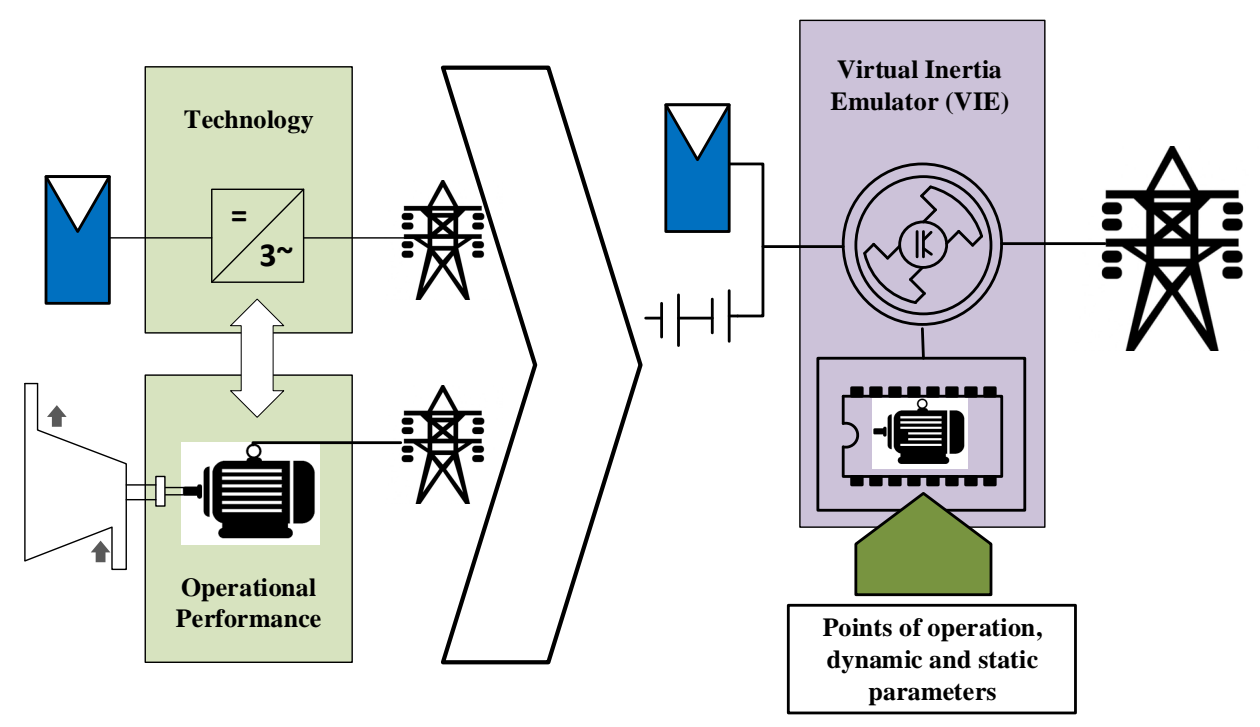

Figure 7.2: Basic idea of the Virtual Inertia Emulator (VIE) [7].

chanical synchronous machines. As illustrated in Fig. 7.2, the properties of an inverter can be programmed in such a method that it executes identical to a synchronous machine between any storage system and/or direct voltage generator and the electric grid.

The storage is connected to the dc-link of the VIE, therefore, it can be worked in a full four quadrant mode and its ac side thus matches the stator output of an electromechanical synchronous machine.

In a real electromechanical machine, the mechanical system provides the energy coupling point with its shaft energy conversion mechanism. However, the mechanical section of a VIE is not a real mechanical system, it exists only as a logical model. Nevertheless, it is completely effective electrically from a grid viewpoint, since it is modeled mathematically in real time by the designed control structure. The VIE process executes in a computer processor or microcontroller, and the physical section is the direct voltage supply circuit of the VIE inverter. In this fashion, a virtual rotating mass is shaped which is effective electrically with regard to the grid. Moreover, it can be can program easily to for any other parameters of a machine. 
Actually, the virtual shaft and the virtual pole system is accessed by manipulating the equivalent factors and parameters in the software that is running on a computer, thus can be implemented locally or through remote dispatch. When active power is required by the grid, a virtual torque on the corresponding virtual shaft must be applied. The necessary energy for this task is taken both from the dc voltage side of the VIE inverter system. Correspondingly, the energy can be supplied to the dc voltage side system by the generation unit. Similarity, when reactive power is needed to be supplied to the grid, the computer changes the virtual excitation voltage values and produce reactive power.By the virtual values of excitation voltage and torque in combination with the power storage, the synchronous machine behavior can be imitated. Close to traditional machines, reactive and active power can be initiated by voltage and frequency variations in the grid, respectively, by remote dispatch. Moreover, the power shortage or excess of the grid areas can be balanced by themselves.

\subsection{Technical Realization}

Modelling the controller for the inverters to imitate the synchronous machine, needs measurement of the current and voltage at the point of common coupling with the grid. In addition, it requires the calculation of the synchronous machine currents in real time and the feeding of the current into the grid.

The designed combination makes the generating of any current profile, such as direct current component, possible. This process is not dependent on the grid voltage but it should be within design specific limits. These current components are necessary for the modeling of the stator current of the virtual machine when a voltage drop happens in the grid. The program interfaces for the machine parameters and the fundamental component management are addressed in each cycle of the algorithm. The on-going calculation of 
the machine model in real time allows a fast response to parameter changes so that newly calculated electrical properties of the machine become effective in the grid immediately.

The controllers, which implemented in a computer program interfaces for the fundamental component management and machine parameters are addressed in real time. This ongoing calculation permits a quick response to changes of parameters in a way that newly calculated electrical properties of the virtual machine would be operational in the grid almost instantaneously.

The reference values of the three-phase current that the inverter injects into the grid, are the machine currents, which was calculated by the implemented program. If the inverter was not able to feed the calculated current value by the machine model into the grid, the properties of the system linearity would be lost. Before starting modeling our virtual inertia modelling, let us take a look at load frequency control in conventional synchronous machines, which is the base of designing of our control system.

\subsection{Different Virtual Synchronous Machine algorithms}

Virtual Synchronous Generators (VSG) or Virtual Synchronous Machines (VISMA) allows the distributed generators to function as a synchronous generator by providing damping properties and short-term inertia of typical synchronous machines. A VSG consists of power electronics inverters (and related control structure) and an energy storage system. By implementing the VSG, the DC source (or renewable generator) will be shown to the network as a synchronous generator in a viewpoint of inertia and damping property. Virtual inertia can be emulated in the weak grid by controlling the active power of the inverter in reverse relationship to the speed of the rotor [24].

Many methods for the VSG systems has been introduced to the literature since 2008. The VSG research team at Kawasaki Heavy Industries (KHIs) [242], the Institute of Elec- 


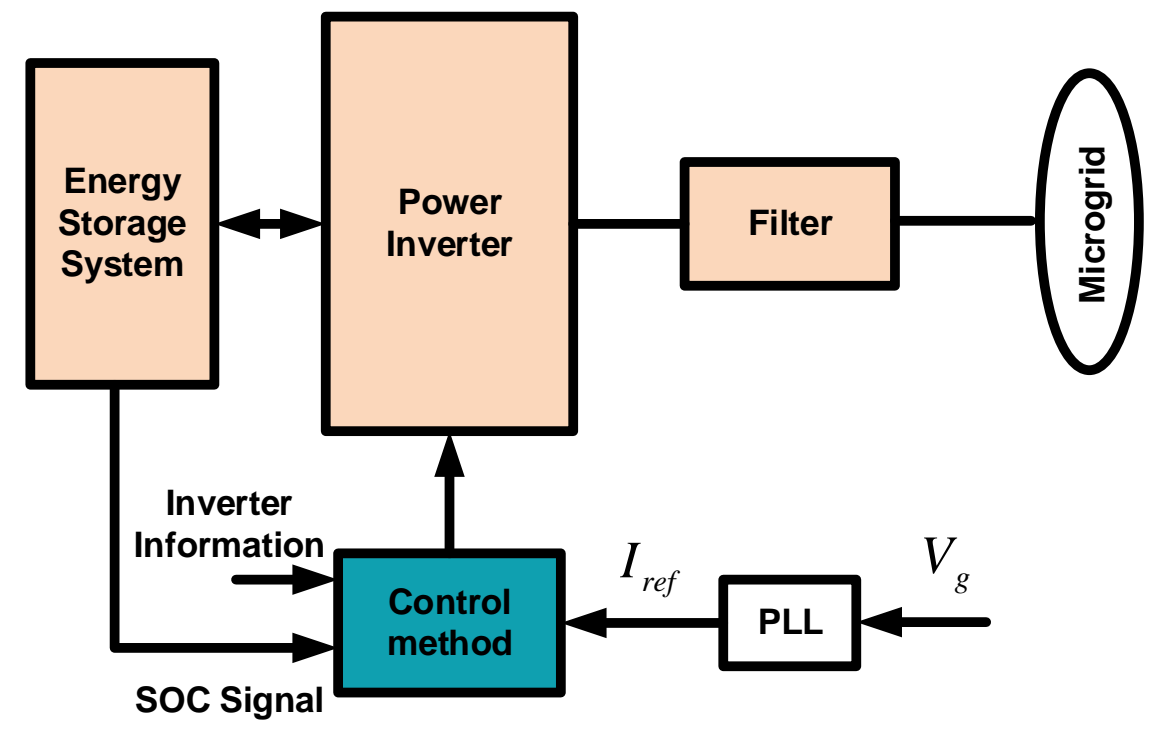

Figure 7.3: The VSG topology by VSYNC that uses the PLL in order to imitate the behavior of synchronous generator .

trical Power Eng. (IEPE) at Clausthal University of Technology in Germany [25, 243245], VSYNC project in the sixth European Research Framework program [246-249], and the ISE Laboratory in Osaka University [250-252] in Japan, are a number of the most notable groups that conduct research in the area of VSG. All of these researches proposed designs to provide dynamic characteristic. In the following section, the brief structures of some established VSG methods are described.

\subsubsection{VSG topology of VSYNC Project}

The VSYNC research group at ECN Intelligent Energy Grids define the idea of VSG system as shown in Fig. 7.3. In this model, the VSG involves of an energy storage system (connected to a DC link), a power electronic inverter, and a grid filter (mostly LCL). The resonant frequency of the filter is designed to be approximately between half of the inverter switching frequency and the nominal frequency of the grid. This topology is commonly used a current mode control for grid currents. 
Current references $\left(I_{r e f}\right)$ are delivered by the Phase-locked loop (PLL) circuit. The PLL circuits are typically used to produce an output signal with the phase is similar to the phase of an input signal or to calculate frequency. However, in this configuration it is exploited to give the reference current of the VSG by using the terminal voltage of the grid $\left(V_{g}\right)$, and the output of the PLL drive the inverter. The electromechanical characteristics of the synchronous generators are emulated by the PLL response that is very similar to the machine. As explained in [253], the phase angle reference of the rotating frame for dq control of the inverter quantities are similarly provided provide by the PLL.

A more comprehensive arrangement of the modified style of this type of VSG is illustrated in Fig. 7.4. All the information required to produce the error current signal ("i dq") is processed by the reference current block. The setting of $K_{S O C}$ must such that the signal of active power $(\mathrm{P})$ is the same as the nominal VSG output power when the deviation of SOC $(\triangle S O C)$ is at its maximum level. Correspondingly, the $K_{v}$ value must be selected so that the maximum reactive power is produced by the VSG for an identified deviation of voltage (e.g., 10\%).

In other publication based on the VSYNC research group [254], the frequency of the grid and the SOC of the batteries (which are collected by monitoring of the exchange of energy between VSG and battery pack), control the DC bus current. In this approach, the frequency is predicted by the zero crossing method, and finally, the set point of current $\left(I_{s p}\right)$ is calculated by the subsequent equation:

$$
I_{s p}=\frac{K_{1} \frac{d \Delta \omega}{d t}}{V_{D C}}
$$

where $\frac{d \Delta \omega}{d t}$ is the rate of frequency change, $K_{P}$ is expressed in $\mathrm{kgm}^{2} / \mathrm{s}^{2}$, and $K_{I}$ is a dimensionless factor. 

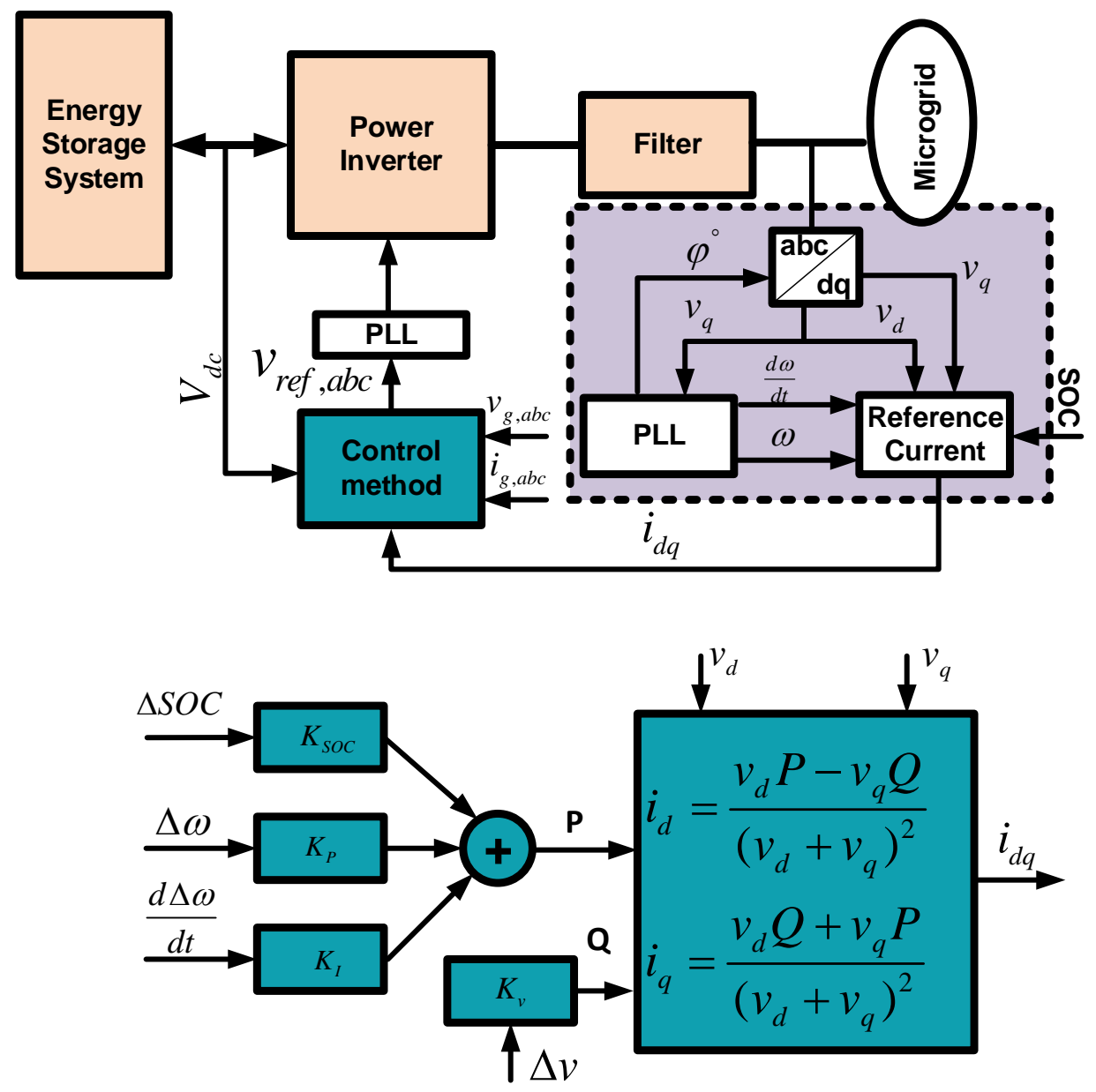

Figure 7.4: structure of the VSG by using PLL, the detailed framework (top), and the block of the reference current(below).

\subsubsection{VSG topology by IEPE}

The dynamic behavior of a simplified synchronous generator model was used as the basic of suggested idea of the VSG in [25,245]. This topology produces the reference voltage and current from the grid current and voltage, respectively. The general VSG structure is presented in Fig. 7.5.

In this scheme, the active and reactive power output of the VSG, damping effect, and the inertia value are set by regulating the parameters of the model by the parameters of 


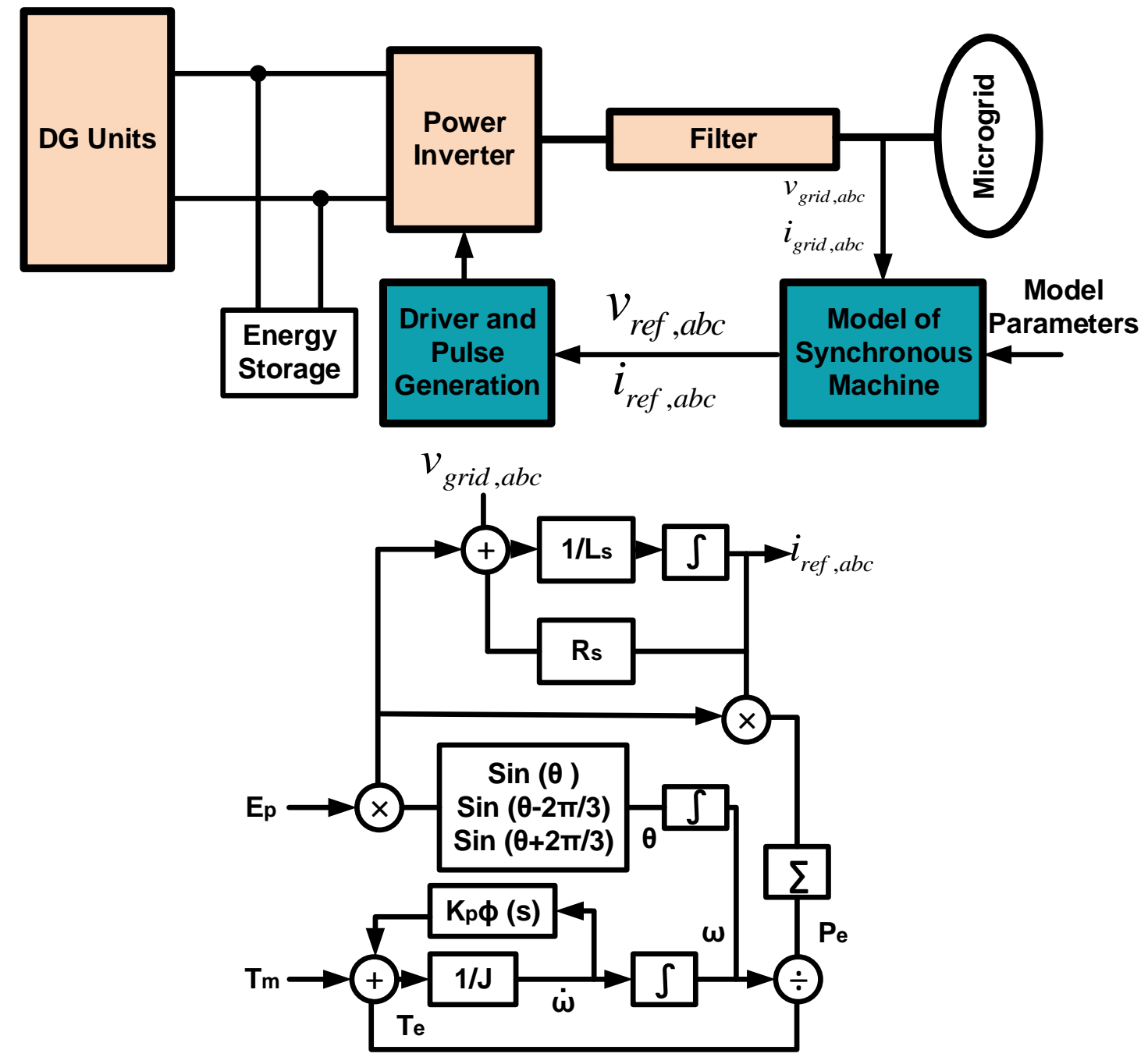

Figure 7.5: The VSG structure based on currentvoltage (voltagecurrent) model of SG: (a) VSG topology, (b) currentvoltage model of SG.

real torque and excitation of a synchronous generator in a power system. The general model of synchronous generator for producing the reference current $\left(i_{r e f, a b c}\right)$ from the signals of the grid voltage $\left(v_{g, a b c}\right)$ is illustrated in Fig. 7.5. In this figure, $\mathbf{R}_{s}$ is the stator resistance, $\mathrm{L}_{s}$ is the stator inductance, $\mathrm{J}$ is the moment of inertia, $\phi(s)$ is the phase compensation term, $\mathrm{K}_{P}$ is the mechanical damping factor, $\theta$ is the angle of rotation, $\mathrm{T}_{e}$ and $\mathrm{T}_{m}$ are the electrical and mechanical torque, and $\omega$ is the angular velocity. The phase 
compensation is implemented in order to ensure the offset of virtual damping force of the opposite phase fluctuating movement of the rotor. In spite of simplifying the excitation winding parameters, the induced electromotive force $(\mathrm{EMF})$ is calculated by the rotation angle $\theta$ and variable amplitude $\mathrm{E}_{p}$.

\subsubsection{VSG topology by ISE lab}

The proposed VSG system by ISE laboratory is presented in Fig. 7.6 [252]. In this structure, the familiar swing equation of a synchronous generator, equation (7.2), is utilized as the main tool for modeling VSG. In (7.2), $P_{\text {in }}$ is the power of prime mover (input power), $P_{\text {out }}$ is output power, $J$ is the rotors moment of inertia, $\omega$ is the virtual rotor angular speed $\left(\Delta \omega=\omega-\omega_{0}\right)$ and $\mathrm{D}$ is the damping factor.

$$
P_{\text {in }}-P_{\text {out }}=J \Delta \omega \frac{d \Delta \omega}{d t}-D \Delta \omega
$$

In each cycle of control process, the swing equation is solved. After that, an integrator receives the frequency $\omega$ to calcite the momentary frequency. Furthermore, PWM pulses are generated by using the virtual mechanical phase angle, $\theta_{m}$.

The power frequency block calculates the grid frequency and the output power, as shown in Fig. 7.6. Based on swing equation, the deviation of virtual angular velocity that is presented by $\Delta \omega_{m}$ is calculated by control block of VSG. Formerly, virtual mechanical phase angle $\left(\theta_{m}\right)$ is used to generate PWM commands.

\subsubsection{VSG topology by KHI}

An algebraic model was used as the heart of VGS model by Kawasaki Heavy Industries (KHI) [242]. The current reference based on the phasor diagram of a synchronous generator is produced by a feedback of the current in order to assure the required functionality 


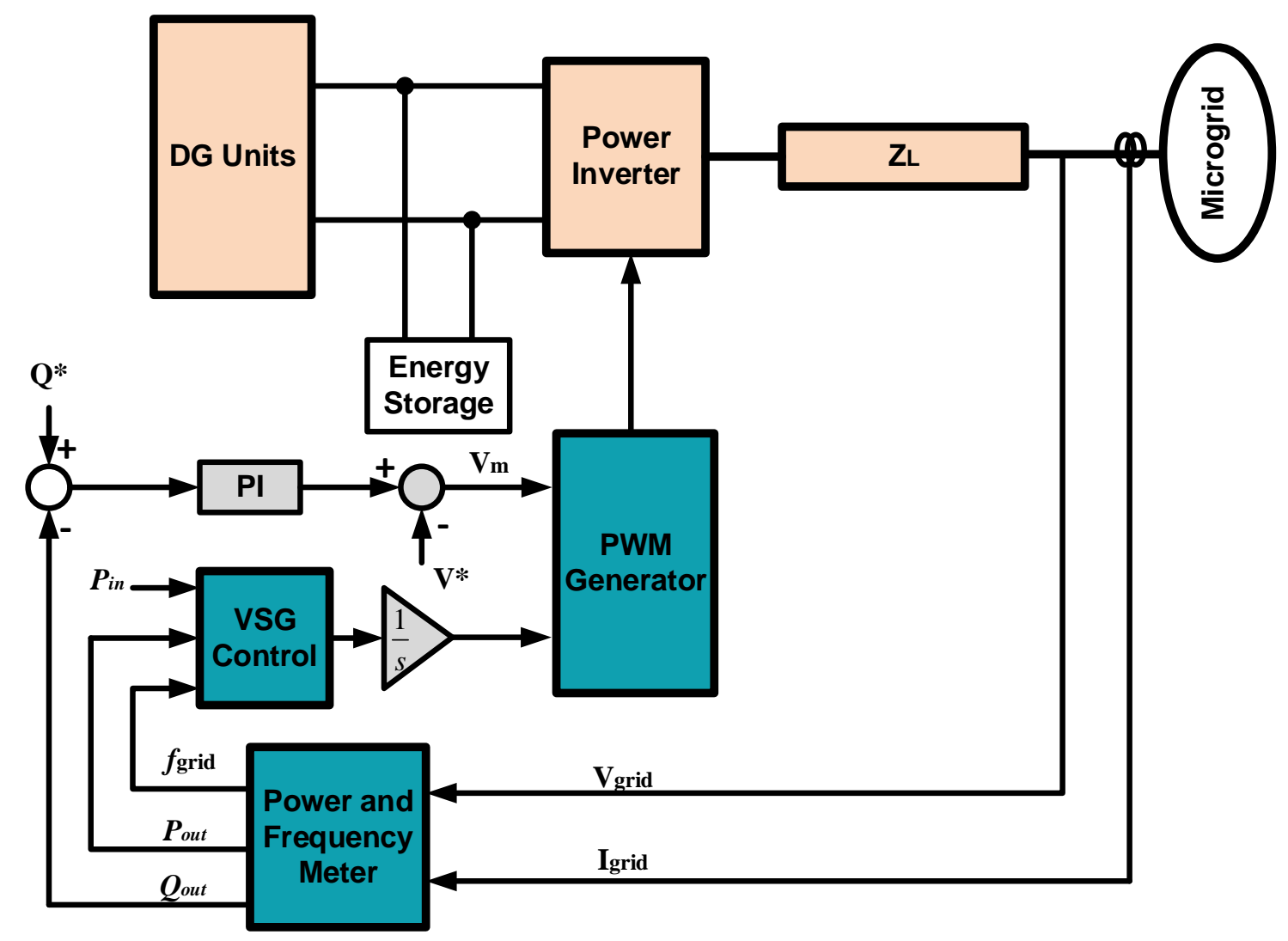

Figure 7.6: The VSG structure proposed by ISE Lab.

of the VSG when nonlinear and unbalanced load types are connected. Moreover, the mitigation of line voltage deviations and angular velocity changes are performed by two more loops. The proposed block diagram of the KHI s VSG is illustrated in Fig. 7.7. In the figure, $\Delta \omega=\omega-\omega_{R}$ and $\Delta P=P-P-0$ are the grid reactive and active power variations, respectively. $\omega_{0}$ is the grid rated frequency (angular velocity). The $\omega$ and $\omega_{R}$ are estimated and virtual rotor frequency (angular velocity) that are calculated by the PLL.

In this figure, $\Delta P=P-P-0$ and $\Delta \omega=\omega-\omega_{R}$ Rare the grid power active and power reactive deviations, respectively. $\omega_{0}$ is the nominal angular velocity (frequency) of the grid. The $\omega_{R}$ and $\omega$ are angular velocity of virtual rotor and estimated by the PLL, respectively. 


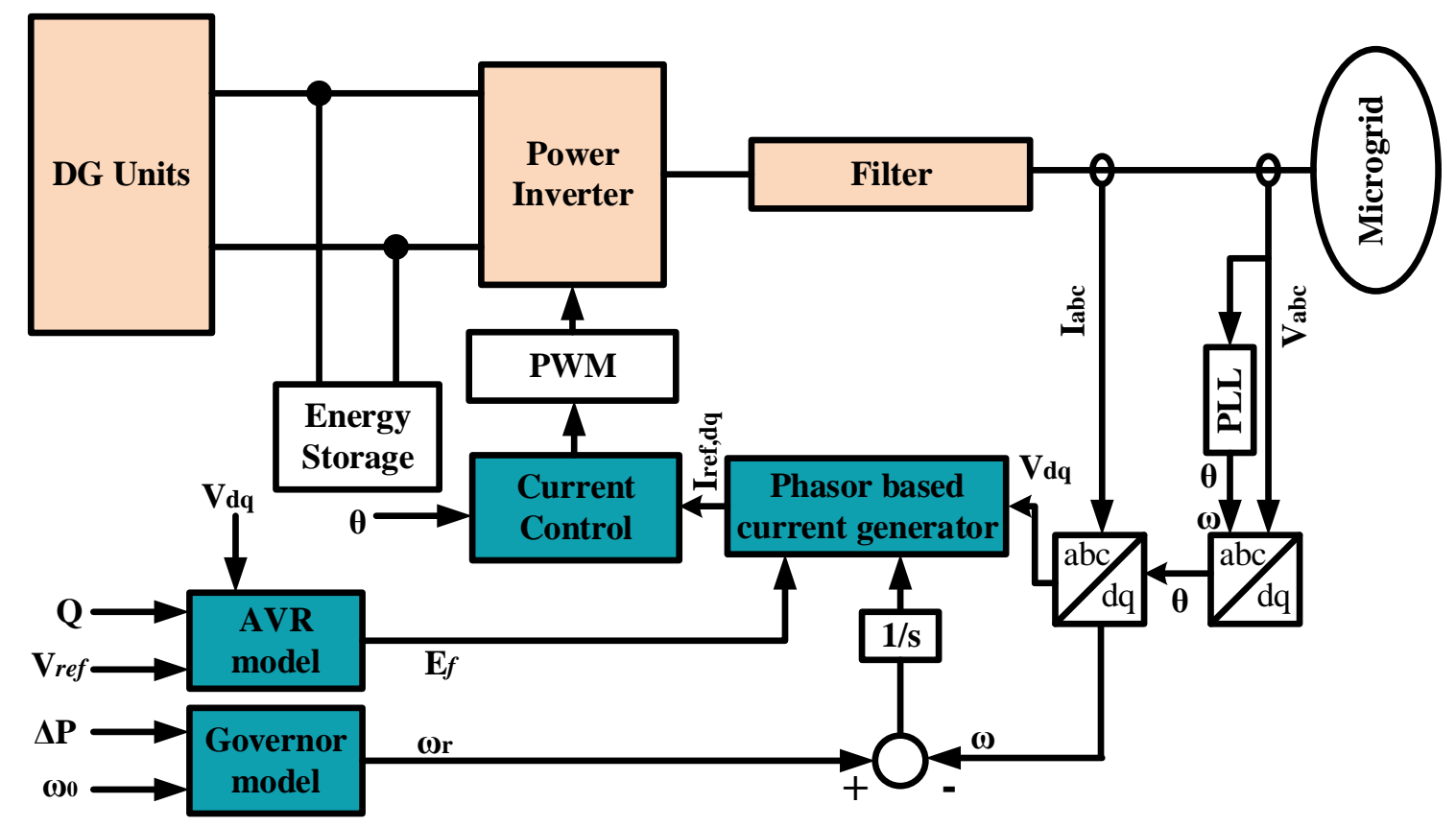

Figure 7.7: The VSG block diagram proposed by Kawasaki Heavy Industries(KHI).

\subsubsection{Typical Method of Implementation of Virtual Inertia Emulator}

\section{in Previous Studies}

The inductance of the filter, $\mathrm{L}_{f}$, has been involved in the internal impedance of the stator windings of the simulated synchronous generator by the inverter. The induced e.m.f. by the rotating rotor flux is denoted by the vector, $e=\left[e_{a}, e_{b}, e_{c}\right]^{T}$. Note that $e$ is the fundamental frequency component of the generated voltage by the inverter.

In a synchronous machine, the electromagnetic quantities consist of the three-phase currents, voltages, and flux linkages in the abc stationary frame are converted to the dq rotational frame. From the machine theory, the electrical model of the synchronous gen- 
erator can be represented by the following equations [255],

$$
\left\{\begin{array}{l}
v_{d}=\frac{d \lambda_{d}}{d t}-\lambda_{q} \cdot \frac{d \theta}{d t}-r i_{d} \\
v_{q}=\frac{d \lambda_{q}}{d t}+\lambda_{d} \cdot \frac{d \theta}{d t}-r i_{q} \\
v_{k d}=\frac{d \lambda_{k d}}{d t}+r_{k d} i_{k d} \\
v_{k q}=\frac{d \lambda_{k q}}{d t}+r_{k q} i_{k q} \\
v_{f}=\frac{d \lambda_{f}}{d t}+r_{f} i_{f}
\end{array}\right.
$$

The flux linkage in (3) is defined as

$$
\left\{\begin{array}{l}
\lambda_{d}=-L_{a d} i_{d}+L_{a d f d} i_{f}+L_{a d k d} i_{k d} \\
\lambda_{q}=-L_{a q} i_{q}+L_{a q k q} i_{k q} \\
\lambda_{k d}=-\frac{3}{2} L_{a d k d} i_{d}+L_{k d f d} i_{f}+L_{k d} i_{k d} \\
\lambda_{k q}=-\frac{3}{2} L_{a q k q} i_{q}+L_{k q} i_{k q} \\
\lambda_{k q}=-\frac{3}{2} L_{a d f d} i_{d}+L_{f d} i_{f}+L_{f d k d} i_{k d}
\end{array}\right.
$$

and the generated real power $P$ and reactive power $Q$ (as seen from the inverter legs) can be defined as

$$
\left\{\begin{array}{l}
T_{e}=\frac{3}{2}\left(\lambda_{d} i_{q}+\lambda_{q} i_{d}\right) \\
P=\frac{3}{2}\left(v_{d} i_{d}+v_{q} i_{q}\right) \\
Q=\frac{3}{2}\left(v_{q} i_{d}-v_{d} i_{q}\right)
\end{array}\right.
$$

Where $\theta$ represents the rotor angle, and $\lambda_{d}$ and $\lambda_{q}$ are the stator flux-linkages reflected in the $d q$ frame respectively. Since a balanced system has been considered in this study, the statements describing the zero-sequence winding circuit are not involved in equations (3) to (5). Also the third and fourth lines in each set of equations in (3) to (5) are used to describe the electromagnetic quantities of the damping circuits associated with the 
rotor, $\lambda_{k d, k q}, v_{k d, k q}$, and $i_{k d, k q}$. In the last line of (3) to (5), $\lambda_{f}, v_{f}$, and $i_{f}$ are the field fluxlinkage, voltage, and current, respectively; $r$ is the resistance of each winding; $T_{e}$ is the electromagnetic torque; $\mathrm{P}$ and $\mathrm{Q}$ are the real and reactive powers; and $\mathrm{L}$ values are the constant inductances. Details of the derivation of (3) to (5) can be found in [256].

$$
\left\{\begin{array}{l}
T_{m}-T_{e}-D_{p} \cdot \frac{d \theta}{d t}=J \cdot \frac{d \theta}{d t} \omega_{m} \\
\omega_{m}=\frac{d \theta}{d t}
\end{array}\right.
$$

where $T_{m}$ is the mechanical torque which can be fixed as a constant value or be ordered to follow a real power demand. In this study the second case is implemented for the purpose of the frequency control; $D_{p}$ is the damping coefficient; and $J$ is the moment of inertia required to imitate the rotational mass of a virtual rotor which is investigated through the integration of a energy storage system.

Implementing nonlinear differential equations 7.3, 7.4, 7.5, and 7.6, which should be solved in real time in the control loop, make the VIE controller very complex . Therefore, it needs the application of many different connected parts that need very high speed processors.

\subsection{VIE Implementation}

In this section step by step design process of the VIE is explained. The main idea of the design came from the Load Frequency Control (LFC) in traditional synchronous generators.

\subsubsection{Load frequency control}

This section is written based on valuable information on [257,258]. Power systems are, basically, synchronous interconnected systems throughout the world. These systems are 
typical synchronous generators that are connected in a parallel manner through the transmission and distribution line, in order to serve the load at the same frequency. This chapter defines the factors of active power and frequency control of the power system in the presence of renewables.

The power generation and load (plus losses) should be matched at every instant of time, or a frequency deviation will happen in the interconnected power system. A small mismatch will cause a small frequency deviation (for example casual load variations) and a large mismatch will cause large frequency fluctuations (for example a large power plant tripping out). The frequency deviation would be positive in case of an excess generation with respect to load or negative in case of a deficient generation with respect to load.

The difference in energy would be stored in the rotating masses of both generation and dynamic load (motors) in the system. Typically, excess mechanical energy in the prime mover (generally a turbine) that will be stored as the generator inertias and cause the acceleration of the moving masses to increase frequency.

With the purpose of understanding exactly how load frequency and control mechanism perform in a real-world interconnected system, it is essential to recognize the connection between the generation inertia (H, in MW-sec/MVA), the magnitude of the disturbances, and size of the system (small or large MW systems) that cause deviations in the frequency.

The minor fluctuations might have various mismatch causes, which can include but not limited to random alterations in load, improper forecasts of load against power dispatch system, fast or slow ramps that are not in synchronism with load variations, and performance of AGC (automatic generation control) causes some differentials that will be corrected eventually. 


\subsubsection{System Modeling}

The typical swing equation for a single generator that is operating by a governor droop implementation is presented. This can be extended to several generators working in parallel. The AGC control is explained by using the two-area model. The concept of system inertia, system droop, and system frequency response to a disturbance are discussed in relation to the area models.

\subsubsection{Dynamics of the System}

Any difference between electromagnetic and mechanical torque will cause acceleration (deceleration) synchronous generator and the prime mover (turbine). Swing equations describes the differential equation between the mechanical and electromagnetic torque:

$$
J \frac{d \omega}{d t}=T_{m}-T_{e}
$$

In $7.7, J$ is the combined moment of inertia of the rotating mass $\left(k g \times m^{2}\right), \omega$ is the angular velocity of the rotor (mech.rad/s); $T_{e}$ is the electromagnetic torque $(N \times m), T_{m}$ is the mechanical torque $(N \times m)$, and $t$ is the time $(s)$.

The inertia constant $(\mathrm{H})$ that is the kinetic energy of the rotor that is stored in rotating mass, (in Joule $=$ Watt $\times$ second) can be defined by normalizing and expressing the equation (7.7) in per unit (p.u.) referred to the generator base rating ( $S_{b}$ in VA):

$$
H=\frac{1}{2} \cdot \frac{J \omega_{0 m}^{2}}{S_{b}}(s)
$$

In (7.8), $\omega_{0 m}$ is the rated angular velocity of the rotor $($ mech.rad/s). Stating the moment of inertia $\mathbf{J}$ from (7.8) as

$$
J=\frac{2 H S_{b}}{\omega_{o m}^{2}}
$$




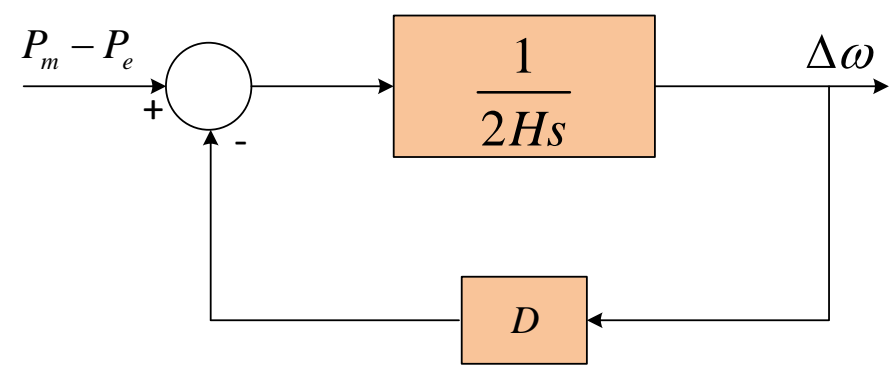

Figure 7.8: Block diagram of the system dynamics plus load damping

and replacing in equation (7.7) obtain

$$
\frac{2 H}{\omega_{0 m}^{2}} S_{b} \frac{d \omega_{m}}{d t}=T_{m}-T_{e}
$$

The difference between the electromagnetic and mechanical torques is represented in the right-hand side of equation (7.10), which is expressed in p.u., and is almost equivalent to the difference between the electrical output power and mechanical input power.

If a disturbance happens in the system (and therefore the system frequency deviates), each of the generators in the system faces a decelerating or accelerating torque. By considering the electromechanical model of the generator:

$$
\begin{gathered}
2 H \frac{d \omega}{d t}+D \omega=T_{m}-T_{e} \approx P_{m}-P_{e} \\
\frac{d \delta}{d t}=\omega_{0} \omega
\end{gathered}
$$

where $2 \mathrm{H}=\mathrm{M}$, and $\mathrm{M}$ is mechanical starting time. Term $\mathrm{D}$ that is the self-regulation of the load in all synchronous areas is typically supposed to be $1 \%$ / Hz. This means that a load reduction of $1 \%$ happens in case of a frequency decrease of $1 \mathrm{~Hz}$. Therefore, in the equation $\mathrm{D}=1$ if load damping is taken.

The block diagram of the dynamics of the system is shown in Figure 7.8. Simplifying the block diagram, the two blocks can be combined into a single forward block using $1 /(2 \mathrm{Hs}+\mathrm{D})$. 


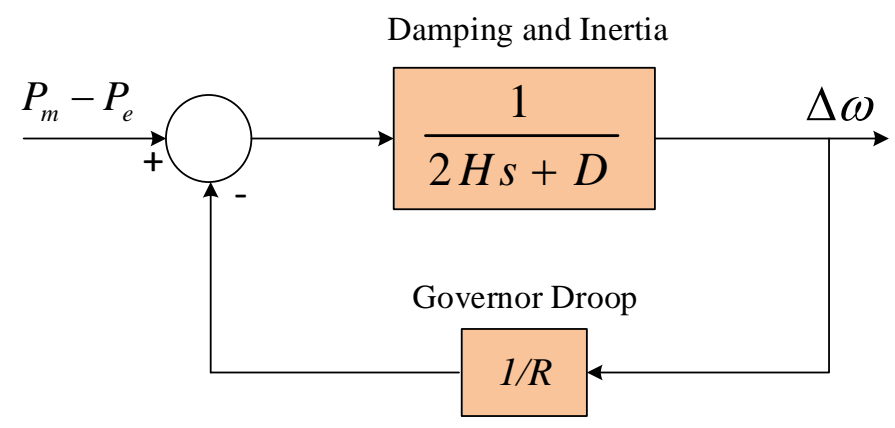

Figure 7.9: Block diagram of the system dynamics plus load damping

The regulation of the unit is defined as droop $\mathrm{R}$ and equals

$$
R=\frac{\Delta \omega}{\Delta P}
$$

The effect of the droop on the regulation of frequency is provided in the block diagram in Fig. 7.9.

\subsubsection{Modelling the Prime Mover}

The prime mover, as the source of mechanical power input, can be gas turbines, hydraulic turbines at waterfalls, and steam turbines. The model for the turbine should give the relationship between the changes in mechanical power output $\Delta P_{m}$ to variations in position of the steam valve $\Delta P_{v}$. Various kinds of turbines have wide different characteristics. Based on recommendations in [258] and [257], the simplest and most practical model for the non-reheat steam turbine prime mover can be estimated by a single time constant $\tau_{T}$ in the range of 0.2 to 20 seconds, which results in the following transfer function:

$$
G_{T}(s)=\frac{\Delta P_{m}(s)}{\Delta P_{V}(s)}=\frac{1}{1+\tau_{T} s}
$$

A simple non-reheat turbine block diagram is illustrated in Fig 7.10. 


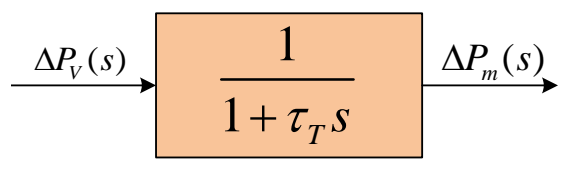

Figure 7.10: Block diagram of simple non-reheat steam turbine

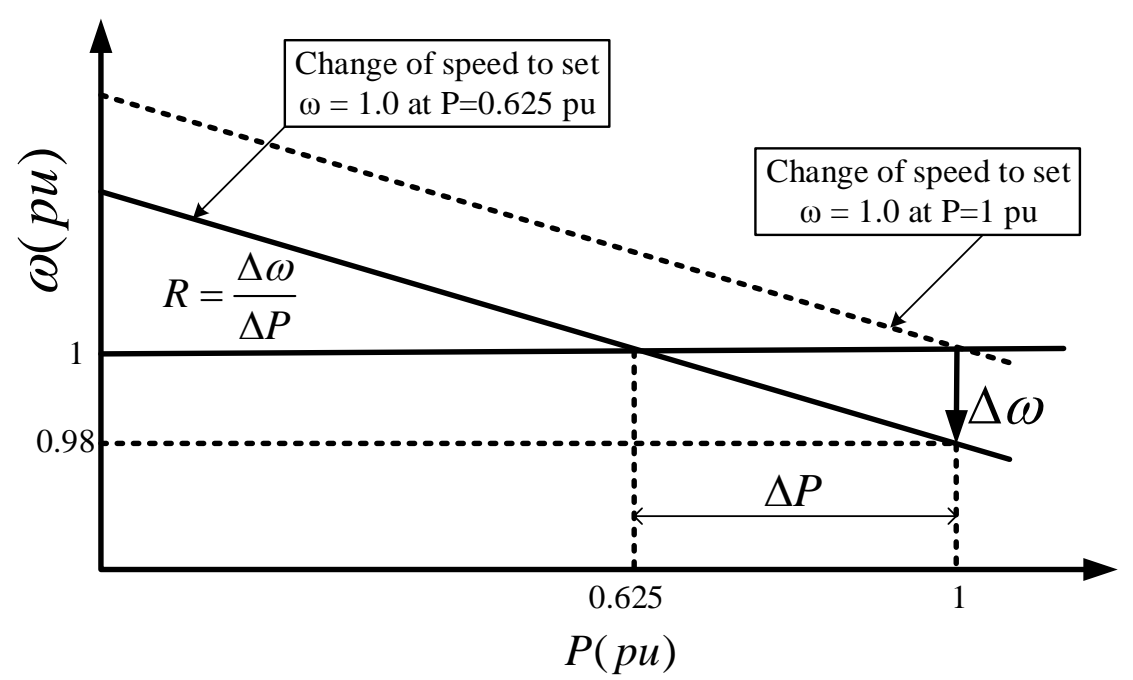

Figure 7.11: Governor steady-state speed characteristics.

\subsubsection{Modelling the Governor}

Suppose that the electrical load of the generator is increased suddenly. This results in the excess of electrical power load in comparison to the mechanical power input. The kinetic energy stored in the rotating system provides the deficiency of power input and power output. The kinetic energy reduction in the system results in the decrease of turbine speed and, subsequently, the generator frequency reduction. In a synchronous generator, the speed change is detected by the governor of the turbine which regulates the input value of the turbine to modifies the mechanical power output in order to set the turbine speed to a different steady-state. Most recent governors execute electronic devices to identify changes in speed. The governors permit a drop in speed as the load increase. The characteristics of steady-state operation of a typical governor is illustrated in Figure 7.11.

The slope of the graph denotes the speed regulation $(R)$. Standard speed regulation of 


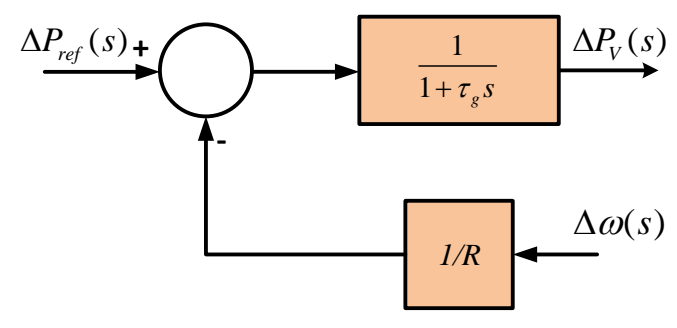

Figure 7.12: Block diagram of speed governing system for steam turbine.

governors, from zero to full load, are 5-6 percent. The mechanism of speed governor is like a comparator with the output is $\Delta P_{g}$, which is the variance of the reference set power of $\Delta P_{\text {ref }}$ and the power $\frac{1}{R} \Delta \omega$ as given from speed characteristics of the governor:

$$
\Delta P_{g}=\Delta P_{r e f}-\frac{1}{R} \Delta \omega
$$

equation (7.15) in s-domain will be

$$
\Delta P_{g}(s)=\Delta P_{r e f}(s)-\frac{1}{R} \Delta \Omega(s)
$$

by considering a linear relationship and assuming a time constant $\tau_{g}$, the following sdomain relation can be given:

$$
\Delta P_{V}(s)=\frac{1}{1+\tau_{g}} \Delta P_{g}(s)
$$

The block diagram of equations (7.16) and (7.17) are represented in Figure 7.12. The block diagrams of Figures 7.9, 7.11, and 7.10, are combined and the result is shown in Fig 7.13 . This figure is the block diagram of the load frequency control of an isolated power generator.

As the VIE process executes in a computer processor or microcontroller, and the physical section is the direct voltage supply circuit of the VIE inverter, in order to reduce the computational calculations, and have a close model to real LFC, the proposed VIE controller is shown in Fig 7.14. In this fashion, a virtual rotating mass is shaped, which is 


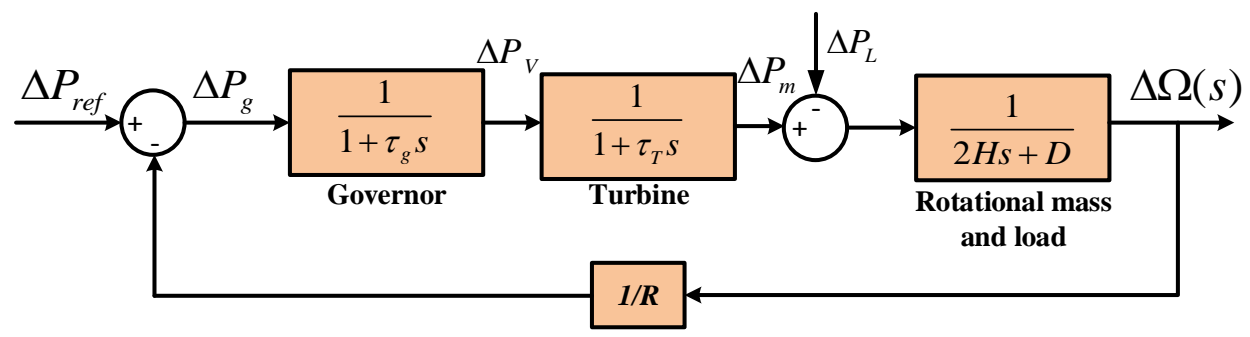

Figure 7.13: Load Frequency control block diagram of an isolated power system.

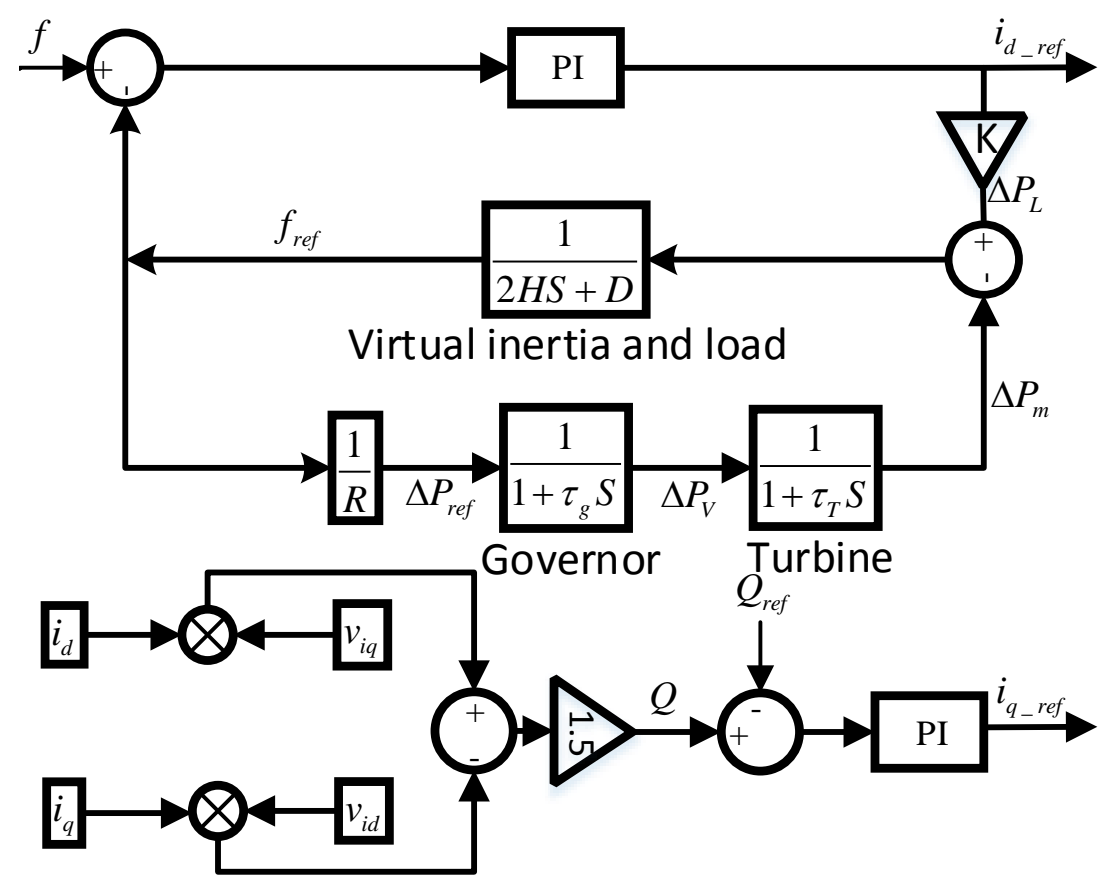

Figure 7.14: Schematic diagram of the VIE model.

effective electrically with regard to the grid. This VIE controller need much more simpler calculations and does not have complex differential equations of the previous studies.

The VIE implemented to meet the objective of maintaining or increase the frequency of grid-connected inverter based renewables to its nominal value. When the self-regulation of the power system is not sufficient to establish a stable state, the frequency of the system will decay continuously until automatic under-frequency load shedding (UFLS) arrests it and reestablish the balance between load and generation during the time constraints in 
Table 7.1: Performance of Battery vs SC

\begin{tabular}{|c|c|c|}
\hline & Supercapacitor & Lead Acid Battery \\
\hline Specific Power Density & $<10,000 \mathrm{~W} / \mathrm{kg}$ & $<1,000 \mathrm{~W} / \mathrm{kg}$ \\
\hline Specific Energy Density & $1-10 \mathrm{Wh} / \mathrm{kg}$ & $10-100 \mathrm{Wh} / \mathrm{kg}$ \\
\hline Discharge Time & $0.3-30 \mathrm{~s}$ & $0.3-3 \mathrm{~h}$ \\
\hline Fast Charge Time & $0.3-30 \mathrm{~s}$ & $1-5 \mathrm{~h}$ \\
\hline Cycle Life & $>500,000$ & 1000 \\
\hline Charge/Discharge Efficiency & $85 \%-98 \%$ & $70 \%-85 \%$ \\
\hline
\end{tabular}

order to prevent the system collapse.

\subsection{Power Smoothing and HESS}

This section describes an algorithm that is designed to decrease the effects of the unpredictability of photovoltaic (PV) power output by exploiting a hybrid battery and supercapacitor storage system (HESS). The main function of the HESS is to add/subtract power to/from the PV output with the purpose of smoothing out the fluctuating power output of PV plants that happens for the duration of shadows of passing cloud on the PV array. The control method is challenged by its ability to reduce short-term PV output variability [259].

The classic energy storage system in standalone PV structure is lead acid batteries. Batteries have low power density and high energy density but, giving low discharge and charge rates. The supercapacitors (SCs) supplies energy using static charge. Compared to the batteries, SC possesses low energy density but high power density, giving high discharge and charge rates. In Table 7.1, the performance of battery and the SC are compared [256].

Passing clouds may cause fast oscillations in the grid voltage, possibly producing following problems: too many operation of load tap changers, voltage regulators, and capacitor banks. For this reason, some codes and standards have been suggested in order to 
regulate the variability of a distributed PV system. For instance, the Puerto Rico Electric Power Authority proposed a constraint to limit the ramping of the PV systems to $10 \%$ per minute.

For a single ramp, the maximum compensation amount of energy is defined by the time that is needed to power ramp from rated power to minimum (or zero) power rapidly or the reverse. It worth mentioning that the batterys time constant is in range of seconds but for the supercapacitor it can be as low as milli seconds or even less. However, a ramp can happen in both directions, therefore, state-of-charge (SOC) of the energy storage system (ESS) would usually be kept close to $50 \%$ to fulfill both charge and discharge requirements. Though, it cause the nominal capacity to double. Owing to the battery inefficiencies a real system would need a battery with a practical size of $25 \%$ to $33 \%$ of the PV array AC size.

Some of the most important energy storage system specifications for smoothing PV power in the literature are as follows [260]:

- Energy Capacity: Practical capacity of the battery bank in a HEES should be between $25 \%$ and $33 \%$ of the rated power of the PV array. It is essential that the energy capacity range is the "useable capacity" of the HESS. For instance, the usable capacity of an HESS with $80 \%$ of maximum depth of discharge of rated capacity, would be the rated capacity $\times 0.8$.

- Power Rating: The HESS is rated equal to the rated output of a PV array for this application. For instance, a $500 \mathrm{~W}$-AC PV system must have a $500 \mathrm{~W}$-AC ESS, which indicates that the rate discharge/charge of HESS is three to four times of the capacity of energy of the HESS.

- Controls: The ESS should be able to handle frequent change between charge and discharge regularly within a few milliseconds. 
However, since our suggested technique focuses on short term fluctuations and includes no long-term power regulation, it needs no mass storage device. In the renewable energy systems with battery alone as energy storage, the PV power produced continuously changes in relation to the changes of temperature and irradiance. When this highly fluctuating and intermittent imbalance power is given to the battery, the battery experiences repeated charging and discharging operations. It increases stress on the battery and it may have harmful effect on the lifetime and performance of the battery. To prevent this, additional energy storage element, super capacitors, are connected to the grid using bidirectional buckboost converter. Since the super capacitor can react faster to quick fluctuations, the stress on the battery can be reduced [261]. Supercapacitors offer a viable solution for power quality upgrading and energy sustainability, as an energy storage device with low power loss, long cyclic life, and high energy density [262]. The Hybrid Energy Storage System (HESS) charges and discharges according to the PV power generation to keep the dc grid voltage constant. The initial SOC of both the battery and super capacitor is set as $50 \%$.

\subsection{Modeling of the Energy Storage System}

An exact model of a hybrid energy storage system for PV power smoothing study is needed to accurately model the whole system. The detailed supercapacitor bank and battery bank models which utilized in our simulation platform are presented in this section [263].

\subsubsection{Modeling the Supercapacitor Bank}

Numerous supercapacitors models have been recommended in the literature so far based on different applications [264-266]. Resistive capacitive networks, achieved by fre- 


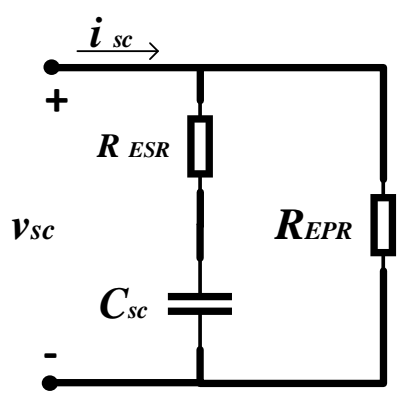

(a)

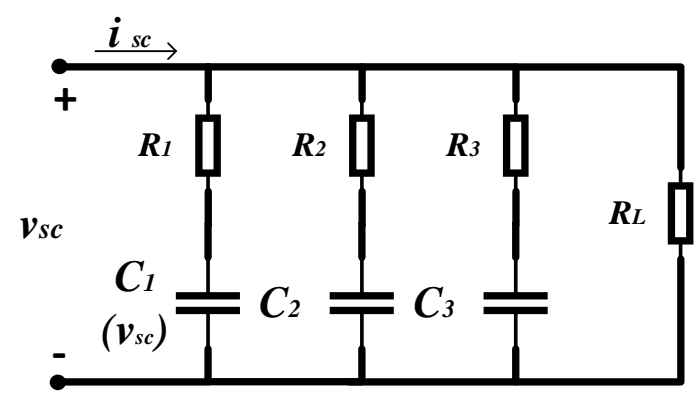

(b)

Figure 7.15: Equivalent circuit of supercapacitor: (a) first order model,(b) third order model by implementing a voltage dependent branch.

quency domain or time domain analysis, typically demonstrate supercapacitors. The most widespread supercapacitor equivalent circuits are shown in Fig 7.15 and Fig 7.16 . The traditional first order estimate model of the supercapacitor is shown in Fig 7.15(a). This model is have three modules: the equivalent resistance $R_{E P R}$, which is a parallel resistor and characterizes the effect of leakage current or the self-discharging, the capacitance $C_{S C}$, which is the capacitance of the supercapacitor owing to the double layer effect, and the resistance $R_{E S R}$, which is the equivalent series resistance and simulates the voltage drop and ohmic loss of the supercapacitor throughout the discharging and charging procedure.

The parameters of the first order model can be easily extracted and the model can be found in the datasheet of the manufacturers, however, the nonlinear fall and rise of the voltage of the supercapacitor, specifically throughout fast discharge and charge, cannot be captured. Consequently, a better-detailed model is shown in Fig 7.15 (b) that is a third order model for supercapacitor using a voltage-dependent differential capacitor in the first branch. In order to reveal the internal charge distribution procedure, each of the branches has a different time constant that will cause easy parameter measurements. The voltagedependent capacitor branch (first branch), shows the instantaneous performance of the 


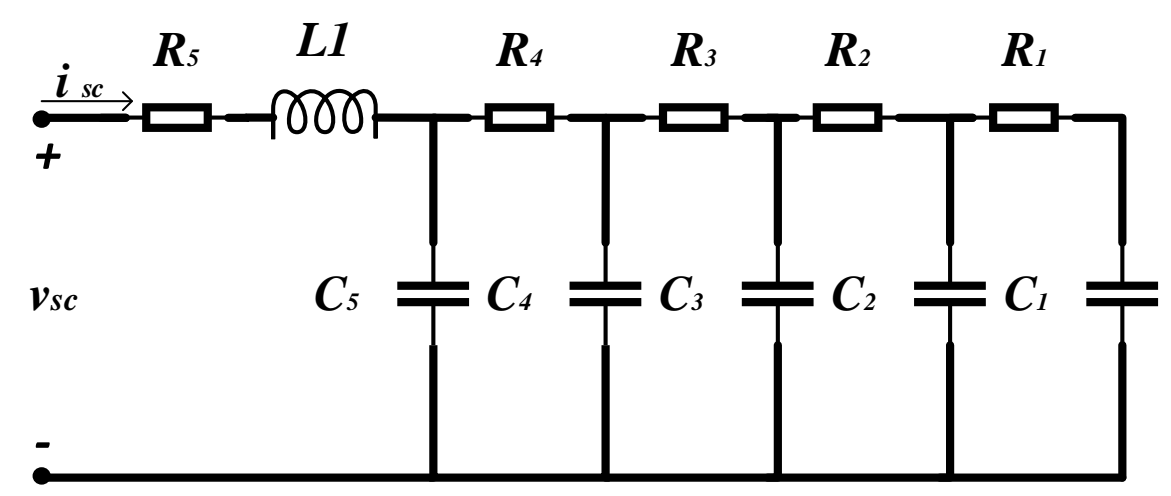

Figure 7.16: Models for supercapacitor bank with a sixth order equivalent circuit.

supercapacitor (in time range of seconds), whereas the second branch determine terminal behavior in the range of minutes and third branch for times longer than 10 minutes. In addition, the last branch has a leakage resistor that models the self-discharge characteristic. Although this model is good for voltages beyond $40 \%$ of the rated dc link voltage and for small frequencies, its accuracy is not enough for lower voltage ranges and high frequencies.

Fig. 7.16 presents a sixth order supercapacitor model that uses nonlinear least-squares fitting and impedance spectroscopy technique. In the electrochemical impedance spectroscopy, method the analysis of the electrochemical cells complex impedance in a widespread range of frequencies from millihertz to megahertz is allowed. This model does not have a unique topology of the circuit for each order, although, it has a very good precision in various applications since it properly spreads each branchs time constant [265]. The time step of the simulation should be less than double of the fastest branch time constant.

The simulation comprises a $6.4 \mathrm{~F}$ supercapacitor bank that supplies high power to the pulsed load instantaneously, so it functions as an energy buffer. The supercapacitor bank is composed of 10 Maxwell's 58-F, 16-V modules and rated $80 \mathrm{~V}$. The specifications of supercapacitor bank are listed in Table 7.2. 
Table 7.2: Supercapacitor bank Specification.

\begin{tabular}{|c|c|}
\hline Parameter & Specification \\
\hline Type & Maxwell (BMOD0058) \\
\hline Rated Capacity & $6.4 \mathrm{~F}$ \\
\hline Maximum Voltage & $80 \mathrm{~V}$ \\
\hline Number of Cells & 10 \\
\hline
\end{tabular}

Table 7.3: Parameters of the sixth order model of the $6.4 \mathrm{~F}$ supercapacitor bank.

\begin{tabular}{|c|c|c|c|}
\hline R1 & $1.02069 \omega$ & C1 & $0.9193 \mathrm{~F}$ \\
\hline R2 & $0.17034 \omega$ & C2 & $1.5428 \mathrm{~F}$ \\
\hline R3 & $0.05069 \omega$ & C3 & $0.5481 \mathrm{~F}$ \\
\hline R4 & $0.05862 \omega$ & C4 & $0.0594 \mathrm{~F}$ \\
\hline R5 & $0.22828 \omega$ & C5 & $0.0008 \mathrm{~F}$ \\
\hline L & $2.2413 \mathrm{E}-3 \mathrm{mH}$ & \multicolumn{2}{|c}{} \\
\cline { 1 - 2 } & &
\end{tabular}

Because the dynamic and the transient features of the dc link in the system is our main purpose, we used the sixth order supercapacitor bank model in our simulation platform. The parameters of the detailed model of a $6.4 \mathrm{~F}$ supercapacitor are presented in Table 7.3.

The total supercapacitor energy that can be supplied is expressed by [239]

$$
E=\frac{1}{2} C\left(V_{\max }^{2}-V_{\min }^{2}\right)
$$

where $V_{\min }$ is its minimum voltage and $V_{\max }$ is the maximum voltage of a supercapacitor bank throughout a pulse load. The maximum variation of dc bus voltage is considered to $5 \%$ in the proposed system.

\subsubsection{Modeling of the Battery Bank}

The lead-acid battery is a nonlinear and complex device. The lead-acid batteries modeling for dynamic operation and transient investigation need the analysis of the battery parameters dependence on the battery storage capacity, battery state of charge, internal heating losses, and self-discharge. In [267,268] an electrical-analytical model for battery is provided that has sufficient precision for a battery energy storage system. This model 
Table 7.4: Parameters of the battery model.

\begin{tabular}{|c|c|}
\hline Parameter & Specification \\
\hline Internal Voltage $\left(U_{0}\right)$ & $60 \mathrm{~V}$ \\
\hline Battery Capacity $(\mathrm{Q})$ & $60 \mathrm{Ah}$ \\
\hline Internal Resistance $\left(R_{\text {batt }}\right)$ & $0.01 \mathrm{\omega}$ \\
\hline Exponential Zone Voltage $(\mathrm{A})$ & $61.0855 \mathrm{~V}$ \\
\hline Exponential Zone Time Constant Inverse $(\mathrm{B})$ & $0.2(\mathrm{Ah})^{-1}$ \\
\hline
\end{tabular}

considered that the voltage of the battery terminals is related to the charge reduction of the battery and internal voltage sue to equation (7.19).

$$
U(q)=U_{0}-K\left(\frac{Q}{Q-q}\right)+A e^{-B q} V_{\text {batt }}=U(q)-R_{\text {batt }} \frac{d q}{d t}
$$

where $U_{0}$ is the internal voltage of the battery, $\mathrm{K}$ is the polarization constant, $\mathrm{A}$ is the exponential zone amplitude of voltage, $\mathrm{Q}$ is the battery capacity, $R_{\text {batt }}$ is the internal resistance of the battery, and B is the exponential zone time constant inverse. The parameters of the detailed model of lead-acid battery bank that is implemented in our simulation model is presented in Table 7.4.

\subsection{Design of Controllers for the HESS}

In the charging and discharging (buck and boost mode) of bidirectional dc to dc converter a merged controller can be used [256]. This means that a single controller can control both of the switched. In this chapter, the boost mode is considered to design the controller.

The block diagram of control strategy of the hybrid battery-supercapacitor is shown in Fig. 7.17. The basic idea of this control strategy is that the battery supports slow transients, whereas supercapacitor supports fast transients. Therefore, the average DC link voltage $\left(V_{d c}\right)$ is compared with reference voltage $\left(V_{r e f}\right)$ and passed through compensator. The compensator gives the total current that is to be supplied from HESS. This reference current $\left(I_{r e f}\right)$ is multiplied by the reference of dc-link voltage to give total power that is 


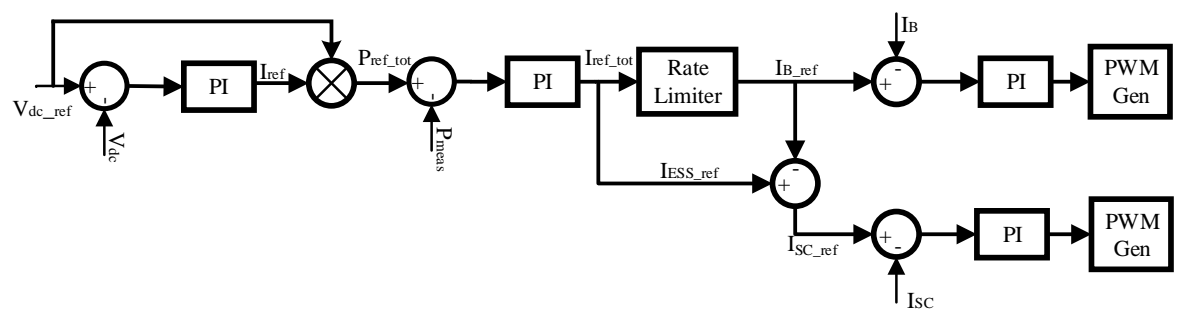

Figure 7.17: Control system designed for a HESS.

to be supplied from HESS $\left(P_{\text {ref }}\right.$ otal $)$. This total power gives is compared with the measured power $\left(P_{\text {meas }}\right)$ and gives the total reference current $\left(I_{r e f_{t} o t}\right)$, which is divided into average current component and dynamic current component using a rate limiter as shown in Fig. 7.17. The average component is given as reference $\left(I_{B-r e f}\right)$ to the battery current control loop, whereas dynamic power component is given as reference $\left(I_{S C-r e f}\right)$ to supercapacitor current control loop.

The supercapacitor has a quicker discharge and charge rates than the battery, therefore, the proportional-integral controllers (PI controllers) are adjusted based on the supercapacitor stage of power. In order to prevent oscillations, the bandwidth (BW) of inner current loop of supercapacitor can not be exceeded by sixth of the switching frequency $\left(f_{s w} / 6\right)$ [269]. With the intention of diverting the transient and fast changing to supercapacitor, the current loop BW of the battery is kept less than current loop BW of supercapacitor, for example at $f_{s w} / 10$. For operation in a stable mode, the BW of the converter is kept much smaller than the right half plane zero frequency of supercapacitor [270]. The calculation of the right half plane zero (RHPZ) frequency is as follows:

$$
f_{\mathrm{RHPZ}}=\frac{\left(1-D_{S}\right)^{2} R}{2 \pi L_{S}} .
$$

The control loop of current is faster than the control loop of the voltage; consequently, the BW of the control loop of voltage is kept less than control loop of current of supercapacitor. The switching frequency is assumed $10 \mathrm{kHz}$ in this study. 


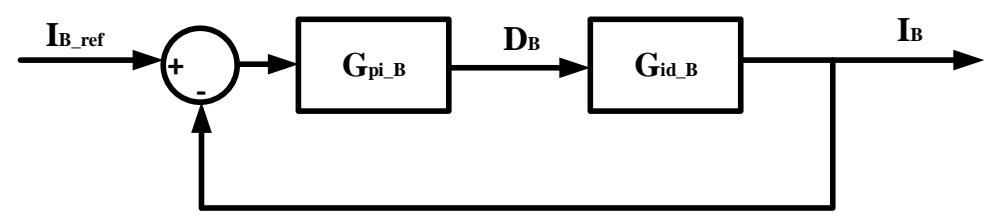

Figure 7.18: The battery bank controller block diagram.

\subsubsection{Design of Current Control Loop of Battery}

Fig. 7.18 illustrates the battery bank controller block diagram. The transfer function of control current loop is given as [271]

$$
G_{\mathrm{id} \_B}=\frac{\tilde{i}_{B}}{\tilde{d}_{B}}=\frac{V_{o} C s+2 \frac{V_{o}}{R}}{L_{B} C s^{2}+\frac{L_{B}}{R} s+\left(1-D_{B}\right)^{2}}
$$

where $\tilde{d}_{B}$ and $\tilde{i}$ are duty ratio of battery converter and small perturbations in current of battery, respectively. $V_{0}$ is dc grid voltage, $\mathrm{C}$ is dc link capacitor, $\mathrm{R}$ is load resistance, $L_{B}$ is the battery inductance, and $D_{B}$ is the duty ratio of the battery dc-dc converter. The current control loop transfer function can be given by

$$
G_{\mathrm{pi} \_}=K_{p-B}+\frac{K_{i_{-} B}}{s}
$$

\subsubsection{Design of Current Control Loop of SC}

Fig. 7.19 illustrates the SC bank controller block diagram. The reference current of supercapacitor $\left(I_{S C-r e f}\right)$ is created by the control loop of voltage. This reference current is provided to control loop of current. The transfer function of current control loop can be given by [271]

$$
G_{\mathrm{id}_{-} S}=\frac{\tilde{i}_{S}}{\tilde{d}_{S}}=\frac{V_{o} C s+2 \frac{V_{o}}{R}}{L_{S} C s^{2}+\frac{L_{S}}{R} s+\left(1-D_{S}\right)^{2}}
$$




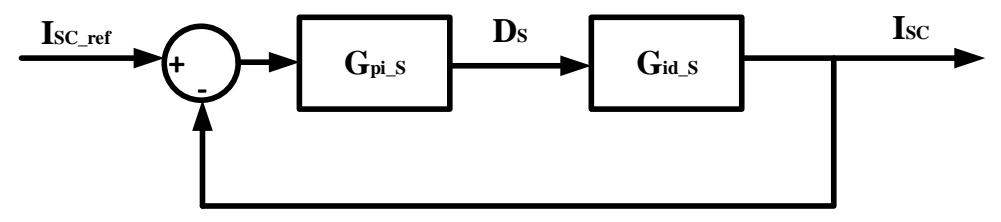

Figure 7.19: The SC bank controller block diagram.

where $\tilde{d}_{s}$ and $\tilde{i_{s}}$ are duty ratio of supercapacitor converter and small perturbations in current of supercapacitor, respectively. $L_{s}$ is the SC inductance, and $D_{s}$ is the duty ratio of the dc-dc converter of the SC.

The transfer function of control loop current can be given by

$$
G_{\mathrm{pi} \_}=K_{p_{-} S}+\frac{K_{i_{-} S}}{s}
$$

\subsubsection{Design of Rate Limiter}

The Rate Limiter block in Fig7.17 performs as a limiter of the first derivative of the signal passing through it. The variations of the output signal is never faster than the indicated limit. The calculation of the derivative is as follows [272]:

$$
\text { rate }=\frac{u(i)-y(i-1)}{t(i)-t(i-1)}
$$

$\mathrm{t}(\mathrm{i})$ and $\mathrm{u}(\mathrm{i})$ are the time and the current block input, and $\mathrm{t}(\mathrm{i} 1)$ and $\mathrm{y}(\mathrm{i} 1)$ are the time and output at the previous step. The output is controlled by matching the rate of change to the Rising slew rate and Falling slew rate parameters[272]:

If the Rising slew rate parameter $(\mathrm{R})$ is less than the rate of change, the output will be:

$$
y(i)=\Delta t \cdot R+y(i-1) .
$$


If the Falling slew rate parameter $(\mathrm{F})$ is bigger than the rate of change, the output will be

$$
y(i)=\Delta t . F+y(i-1) .
$$

If rate of change is between the values of $\mathrm{F}$ and $\mathrm{R}$, the output change will be equal to the input changes:

$$
y(i)=u(i)
$$

The block output at the start time $(\mathrm{t}=0)$ is considered as if rate of change is not inside the bounds of $\mathrm{F}$ and $\mathrm{R}$. At the start time $(\mathrm{t}=0)$, the rate is calculated as follows:

$$
\text { rate }=\frac{u(0)-y(-1)}{t_{s}}
$$

where $t_{s}$ is the sample time of simulation.

\subsection{System Description}

As illustrated in Fig. 7.20, to allow the frequency control function of the PV system a HESS is added to the grid-tied inverter. The inverter is controlled to imitate a synchronous generator in its ability to deliver inertia to the electric power grid. This form of inverter control is also known as VIE. In this chapter, the topology of a VIE principally contains VIE controller with governor, turbine and iertia model, provided in Fig. 7.14, the voltage controller, and the output filter, as shown in the dotted box in Fig. 7.20. In the VIE, the stator terminal voltage of the virtual synchronous generator, $v=\left[v_{a}, v_{b}, v_{c}\right]^{T}$, are represented by the voltages across the filter capacitors.

A grid-connected PV system consisting of PV panel, battery, and supercapacitor arrangement with a virtual inertia emulator and their related controllers is shown in Fig. 7.20. The PV panel is connected to dc bus using a boost converter. Here, the boost converter is used to extract the maximum power from PV panel by using maximum power 


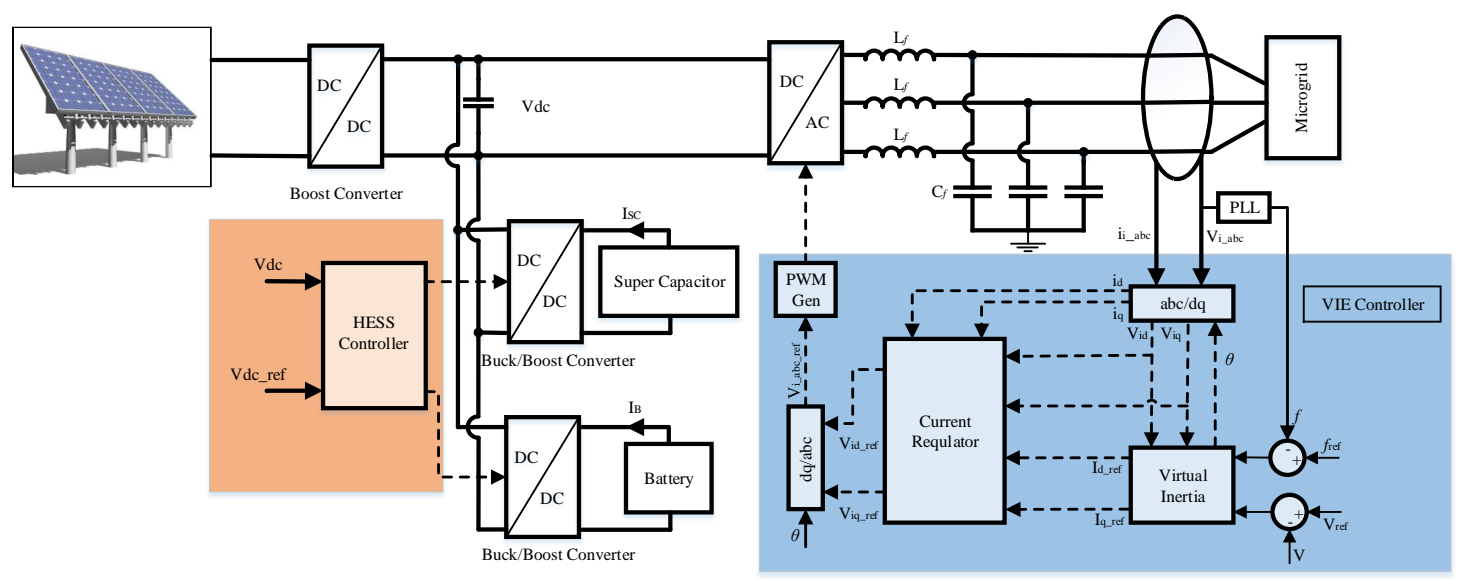

Figure 7.20: An overview of the proposed system a)Hybrid Energy Storage Controller b)Virtual Energy Inertia Controller

point tracking (MPPT) algorithm. HESS is connected to dc bus using bidirectional dc/dc converters. HESS is used to maintain the constant dc bus voltage $\left(\mathrm{V}_{d c}\right)$ due to intermittency of solar irradiance.

The grid-interfaced inverter is controlled to convert the DC to alternating current and regulates the real and reactive powers flowing into the grid instantaneously and autonomously. The inverter controller can be designed to simulate traditional generators, as is the case to be presented in this chapter.

\subsection{Simulation Results}

To confirm the usefulness of the proposed control configurations, a grid connected PV system with VIE and HESS controller was modeled in Matlab Simulink. The PV system consists of $500 \mathrm{~W}$ generating modules. The PV model uses the simulated solar irradiation data with high fluctuation in 15 seconds (Fig 7.21). The study conducted in two scenarios. In the first scenario, the high ramp rate fluctuations of $\mathrm{PV}$ is modeled and the effect of designed HESS controllers was analyzed. In this scenario, the load remained unchanged. In the second scenario, it was assumed that the PV has a constant output and a 1.2 pu load 


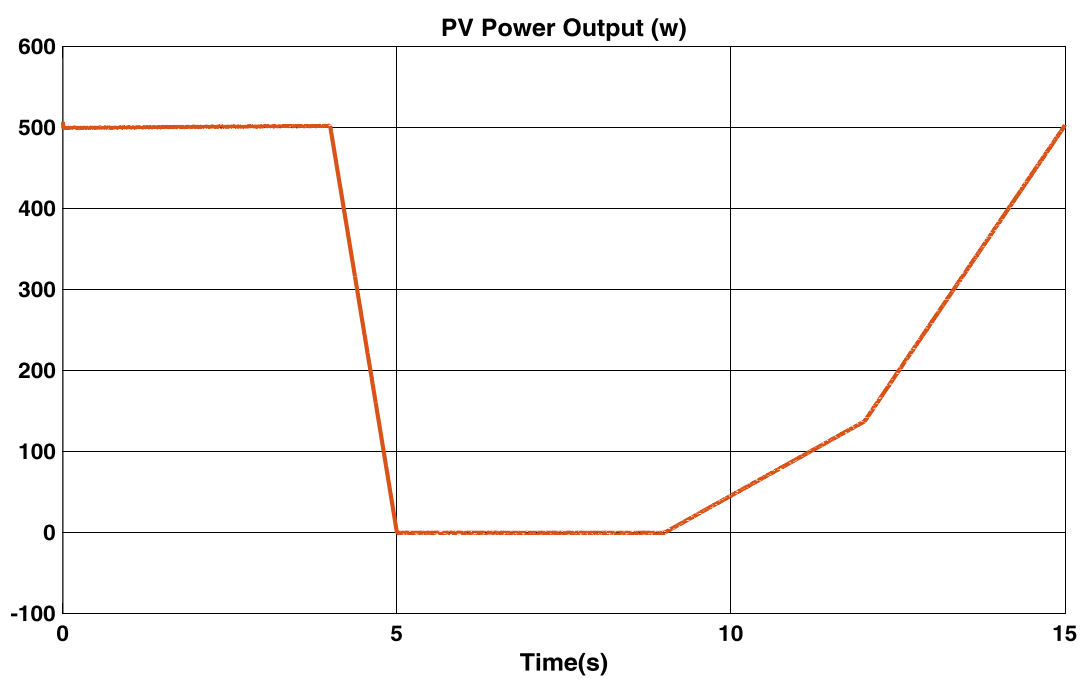

Figure 7.21: The output power of photovoltaic arrays .

connected at the point of common coupling (PCC). The dc bus has been regulated to a constant voltage of $320 \mathrm{~V}$.

Furthermore, the state of charge of battery has initially adjusted at $50 \%$. In this model a 500w three level bridge inverter with a carrier-based three level PWM controller has been implemented. The dc link has used a 6 milli farad capacitor. The sampling time of the simulation is set to $25 \times 10^{-6}$ seconds. The values of the $\mathrm{LC}$ filter are $\mathrm{L}=1 \mathrm{mH}$ and $\mathrm{C}=6.15 \mu \mathrm{F}$. In this model a generic battery that model most popular battery types has been used. The rated capacity of the batteries are $12 \mathrm{Ah}$, with nominal voltage of $12 \mathrm{~V}$. The number of series batteries are 5. The supercapacitor model in this simulation is a generic model of Electric Double Layer Capacitors (EDLCs) with rated capacity of $58 \mathrm{~F}$ and rated voltage of $16 \mathrm{~V}$. The capacitor bank consists of two sets of five series capacitors in parallel. 


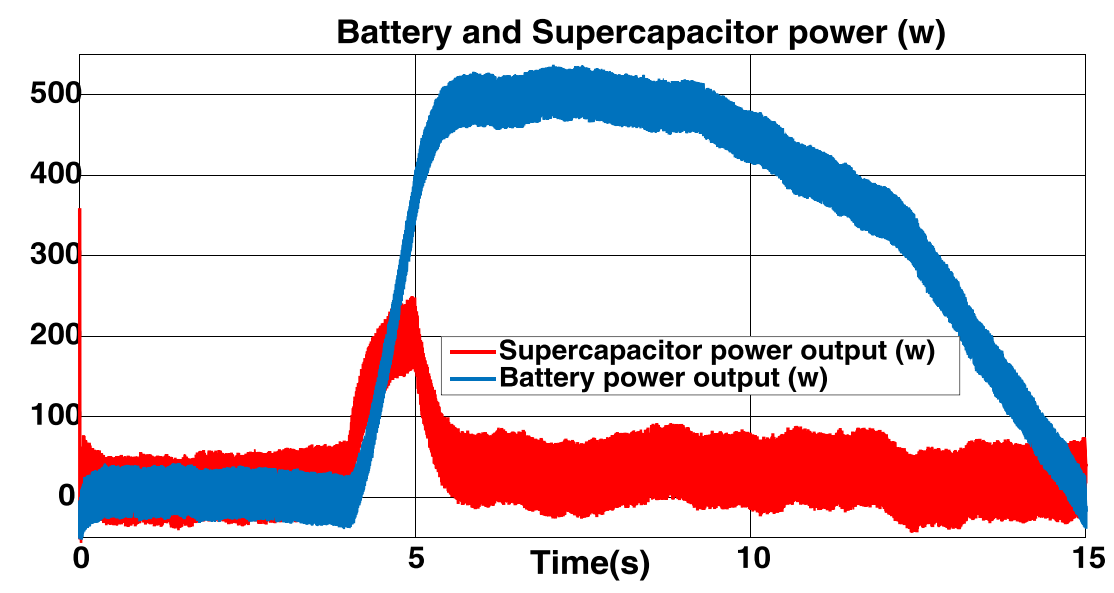

Figure 7.22: Battery and Supercapacitor power output.

\subsubsection{Performance of HESS in high ramp PV output fluctuations}

In the first part of the simulation, the load was considered constant and the mitigation of the solar intermittency is the purpose of the study. The battery and supercapacitor charges and discharges according to the PV intermittency to keep the dc grid voltage constant. The terminal voltage of the battery and supercapacitor will fluctuate depends on the PV output power imbalance nature. The power output of the battery and supercapacitors in the combined energy storage mode is illustrated in the Fig 7.22. It can be observed from this figure that the high-frequency rise and fall appearing in Fig. 7.21 are mostly accommodated by the super-capacitors. In this study, the worst case scenario was considered by a surge change in power output at $\mathrm{t}=4 \mathrm{~s}$. At this time, the PV output became zero in one second. At this time supercapacitor compensates the high power fluctuation ramp rate by injecting power in a short period of time. Then the power output remained zero from $t=5 \mathrm{~s}$ to $\mathrm{t}=9 \mathrm{~s}$.

As it can be seen, the battery start injecting power with a lower ramp rate at $t=4 \mathrm{~s}$ and then continue injecting power at an approximately constant power of 500 watts until $t=9 \mathrm{~s}$. As the PV power output start increasing, the battery power decreases with a slow 
rate of $50 \mathrm{w}$ per seconds until $\mathrm{t}=12 \mathrm{~s}$. From $\mathrm{t}=12 \mathrm{~s}$ to $\mathrm{t}=15 \mathrm{~s}$, the battery power output rate increases to $116.67 \mathrm{w}$ per seconds, and finally it reaches zero at $\mathrm{t}=15 \mathrm{~s}$. A supercapacitor does not have any activity after the high PV power drop of 500w per seconds. Therefore, currents with low variations are taken by the battery units within the HESS. Using HESS has another advantage of increasing the life expectancy of the battery pack by directing the high-frequency fluctuation to the supercapacitor. Fig. 7.23 illustrates the SOC of the battery bank and supercapacitor bank during short term operation of the system. When the PV power output is maximum, during $0 s<t<4 s$, SOC of both battery and supercapacitor are increasing. At $\mathrm{t}=4 \mathrm{~s}$, the PV power output ramp down sharply and reaches Ow in one second. The SOC of the battery stop increasing in less than a second and the battery discharges from $t=5 \mathrm{~s}$ to $\mathrm{t}=12 \mathrm{~s}$. On the other side, the supercapacitor discharges $0.843 \%$, but start charging again since it is only responsible for the fast ramp rates. The battery starts changing after $t=15 \mathrm{~s}$, when the PV power output increased enough to meet the load.

The main purpose of HESS is to keep the dc-link voltage constant. Fig. 7.24 shows that the dc-link voltage variation during the PV fluctuations is an acceptable range of $\pm 5 \%$. To be more precise, the dc-link voltage drops only $1 \%$ or $3.1 \mathrm{v}$ during the time that PV output drops from maximum power to zero. Fig. 7.25 illustrates the total power output of the HESS. This figure shows how the sum of battery and supercapacitor, with a maximum of $6 \%$ error or $30 \mathrm{w}$, is equal to the power deficiency of the PV power.

\subsubsection{Dynamic operation of Virtual Inertia Emulator}

For the second part of the simulation, the DC voltage is considered as constant and the effect of a pulsed load connecting into the grid is analyzed. In this simulation, the incremental load change occurs at $\mathrm{t}=2 \mathrm{~s}$, and its value changes from $500 \mathrm{w}$ to $1100 \mathrm{w}$. This load 


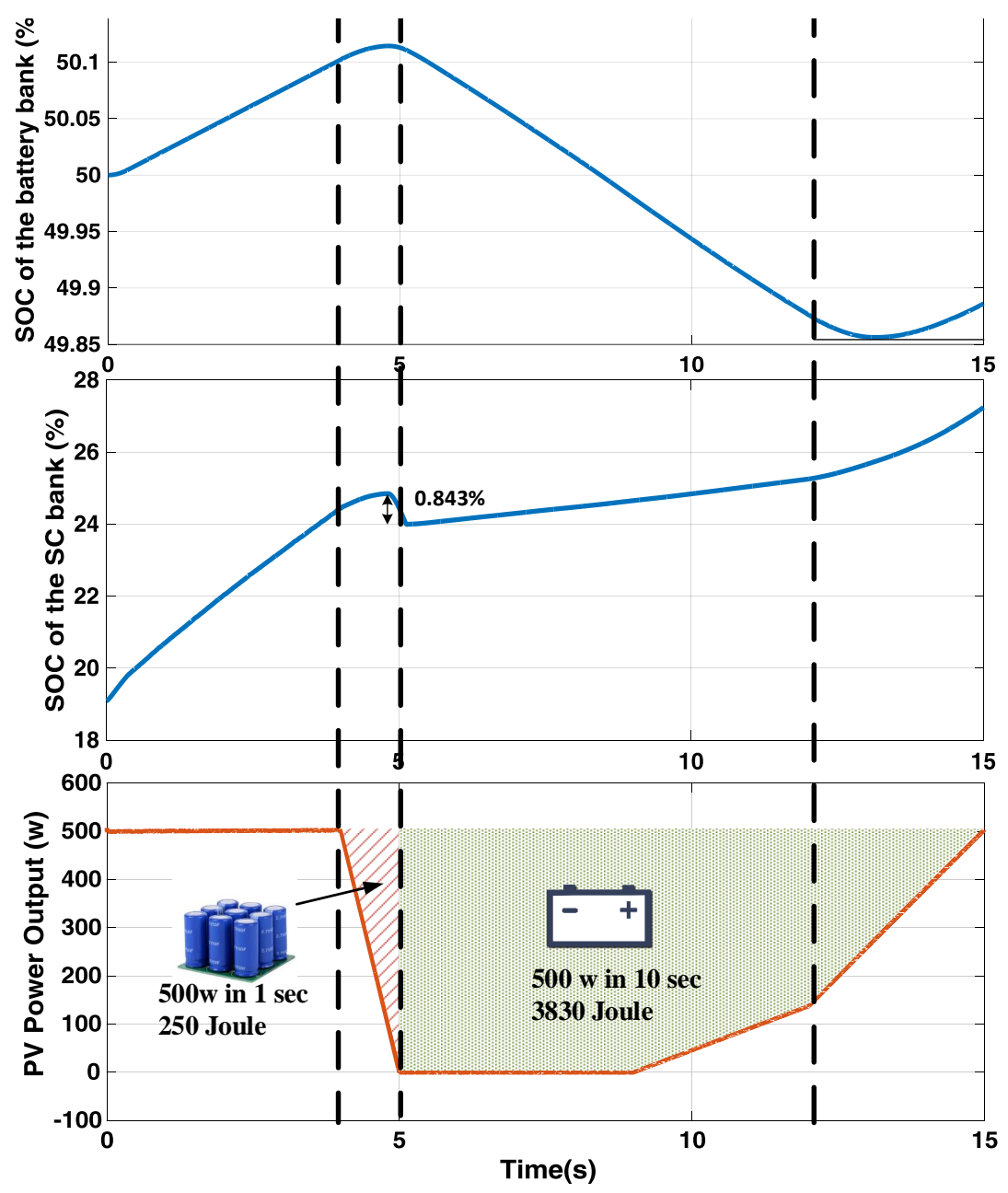

Figure 7.23: SOC of the battery banks and Supercapacitor bank during intermittencies.

will be disconnected at $\mathrm{t}=2.5 \mathrm{~s}$ which causes frequency variations at the time of connection and disconnection. In this case, the VIE-based control scheme delivers inertial response support. For this study, we assume that the inertia constant $(\mathrm{H})$ has four different values of $\mathrm{H}=10 \mathrm{j} / \mathrm{VA}, \mathrm{H}=30 \mathrm{j} / \mathrm{VA}, \mathrm{H}=50 \mathrm{j} / \mathrm{VA}$, and $\mathrm{H}=70 \mathrm{j} / \mathrm{VA}$ and the frequency variations are illustrated for all four values in Fig 7.26. With higher values of $\mathrm{H}$, the frequency at the point of common coupling (POC) dropped with a smaller amount. When the $\mathrm{H}$ has a low value of 10 , the frequency drops to $59.83 \mathrm{~Hz}$ at $\mathrm{t}=2 \mathrm{~s}$. However, by increasing the inertia constant the frequency have fewer fluctuations, for example, by the highest inertia 


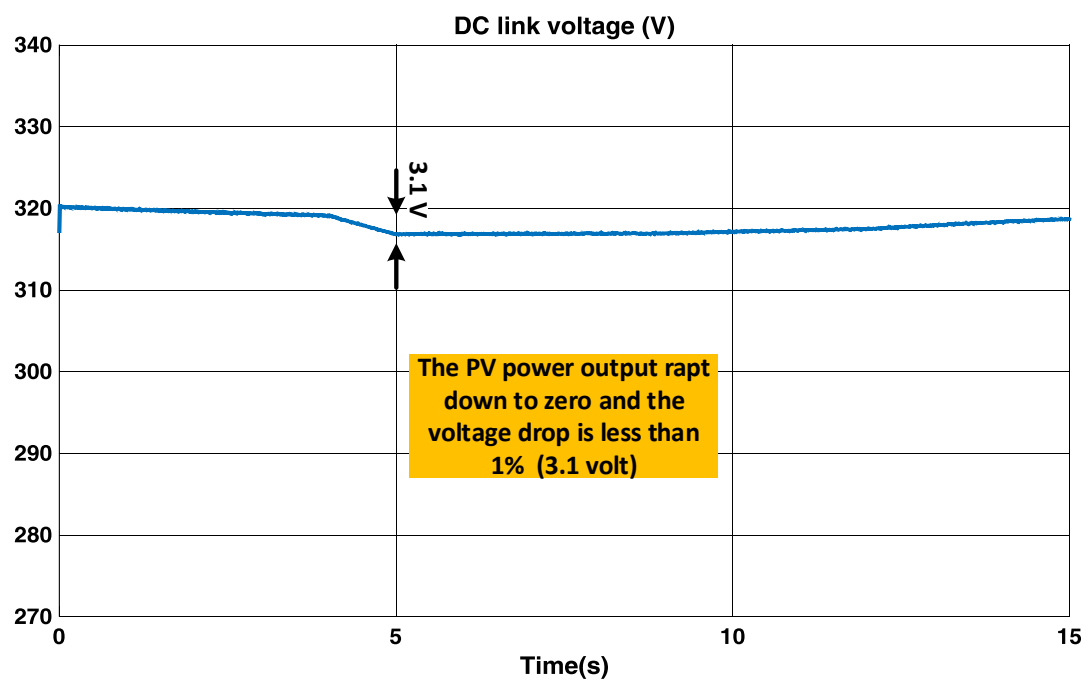

Figure 7.24: DC link voltage variation by solar intermittency.

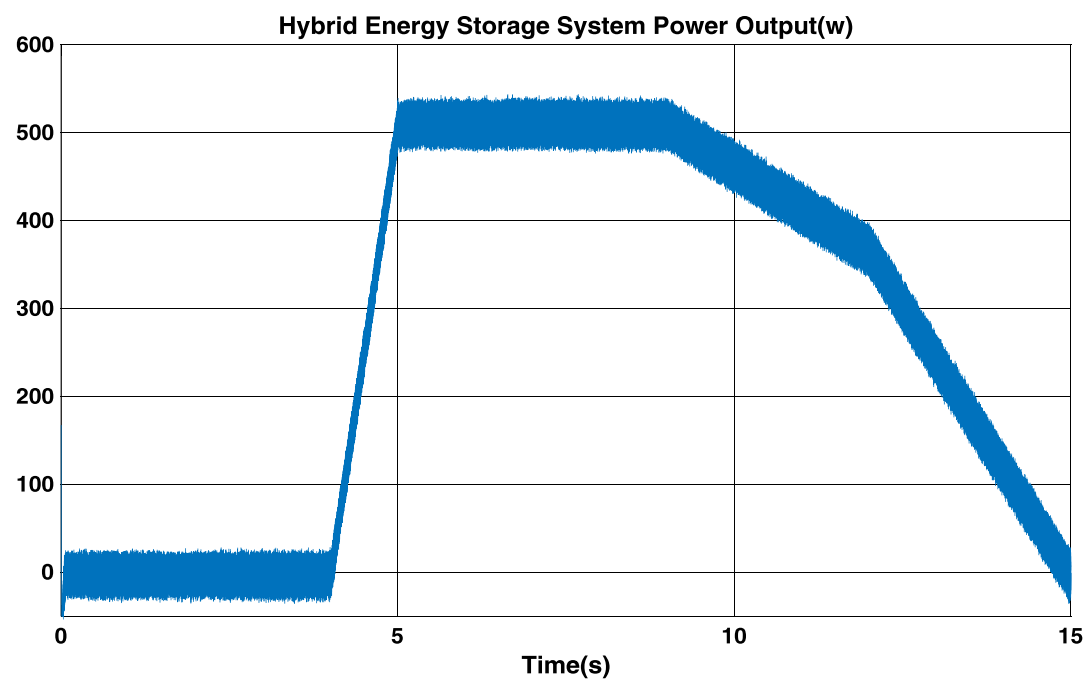

Figure 7.25: Total power output of Hybrid Energy Storage System (HESS).

constant $(\mathrm{H}=70 \mathrm{j} / \mathrm{VA})$, the frequency drops to $59.88 \mathrm{~Hz}$ and the error have been improved from $0.17 \mathrm{~Hz}$ to $0.12 \mathrm{~Hz}$. At $\mathrm{t}=2.5 \mathrm{~s}$, the lowest inertia does not result in the highest frequency deviation, however, when $\mathrm{H}=70 \mathrm{j} / \mathrm{VA}$ (highest value of this study), the frequency has an overshoot of $60.10 \mathrm{~Hz}$, which is less than other cases.

The simulation results of reference currents and voltages in synchronously rotating 


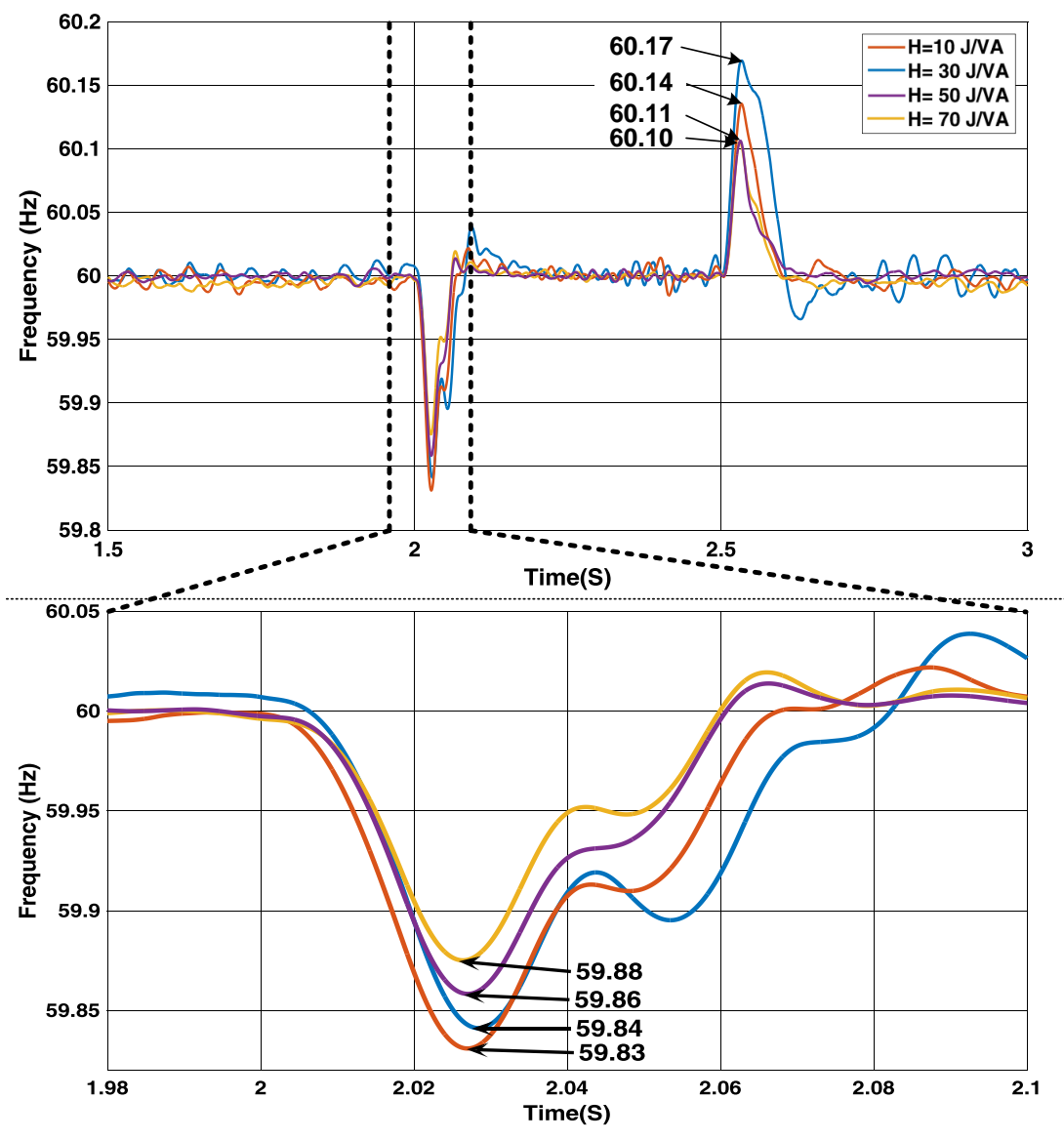

Figure 7.26: Variation of Frequency at POC while a big load connects at $t=1 \mathrm{~s}$ and disconnects at $\mathrm{t}=2.5 \mathrm{~s}$.

reference frame are shown in Fig 7.27. It can be seen how the per unit values of the $I_{d}$ and $I_{q}$ change to maintain the voltage and frequency constant when $2 \mathrm{~s} \leq \mathrm{t} \leq 2.5 \mathrm{~s}$.

Fig 7.28 shows the variation of active and reactive power during pulsed load event. At $\mathrm{t}=2 \mathrm{~s}$ and $\mathrm{H}=70 \mathrm{j} / \mathrm{VA}$, both active and reactive power are increased to mitigate the load increase. Active power rises from $411 \mathrm{w}$ to $437.9 \mathrm{w}$ and reactive power surges from zero to 47.55 Var to compensate the voltage drop. After a peak of $437.9 \mathrm{w}$ and providing enough inertia to the grid, active power starts decreasing to $371.5 \mathrm{w}$ at $\mathrm{t}=2.5 \mathrm{~s}$. It worth mentioning that the PV provides $45.5 \%$ of the load and the rest is injected by the grid. In in contrast to active power, reactive power increases to 101.6Var during pulsed load event to keep the 


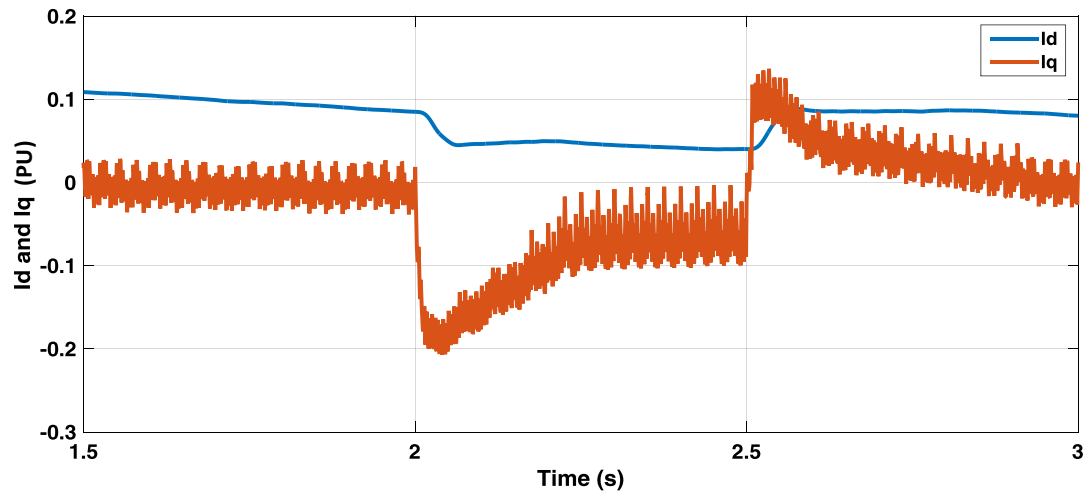

Figure 7.27: Variation of IdIq references while a big load changes at $\mathrm{t}=1 \mathrm{~s}$.
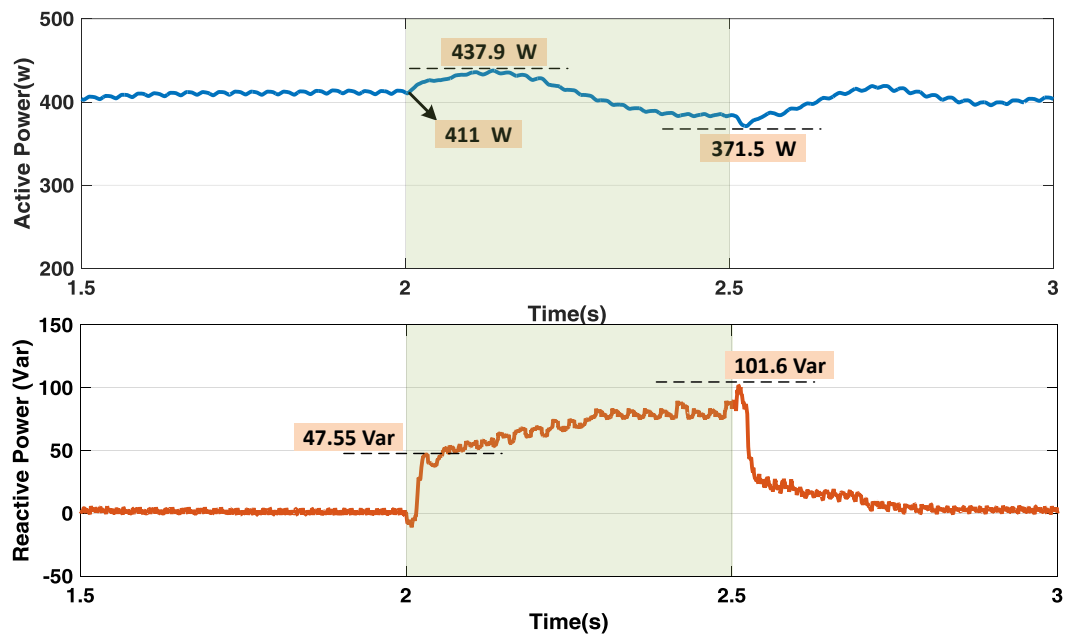

Figure 7.28: Active and Reactive power variation during pulsed load event.

voltage at acceptable 5\% range.

As it can be seen in Fig. 7.29, implementing virtual inertia emulator helps the voltage remain constant during $2 \mathrm{~s} \leq \mathrm{t} \leq 2.5 \mathrm{~s}$. The RMS voltage without VIE drops to $110.8 \mathrm{v}$ at $\mathrm{t}=2 \mathrm{~s}$, and has an overshoot of $127.6 \mathrm{v}$ at $\mathrm{t}=2.5$. However, these vales are $118.9 \mathrm{v}$ and $121.1 \mathrm{v}$ when VIE is applied to the system. Therefore, the voltage violation improves by a factor of $6.75 \%$ at $\mathrm{t}=2$ and $5.41 \%$ at $\mathrm{t}=2.5 \mathrm{~s}$. 


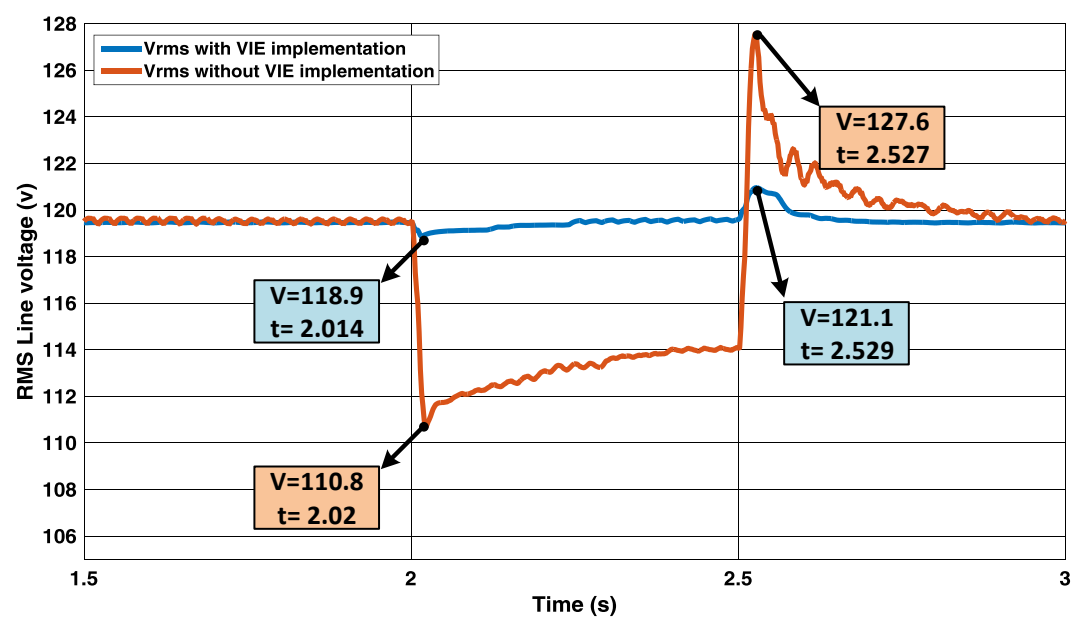

Figure 7.29: Voltage variation when a puled load is switched in.

\subsection{Summary}

The VIE idea as offered in this chapter makes it easier to connect renewable electrical generators, such as PV into weak grids. Owing to the similarity of the proposed system to the electromechanical synchronous machine: 1- all the usual dynamic and static properties of the conventional grid operation is possible. 2- Parallel operation with the grid is possible with any number of generators and without supplementary lines for communication. Another objective of the VIE development is to create a power station by designing control system of a virtual machine. The proposed system also offers the advantages of changing the parameters of the system throughout the operation.

Moreover, a hybrid energy storage system (HESS) including a battery energy storage system (BESS) and a super-capacitor is assessed in this study. This system is designed to accommodate fast solar power fluctuations because of the intermittent nature of irradiance situations. Also, as conventional generators are replaced by high-penetration levels of renewable energies (mostly PV generation), the existing rotating kinetic energy in the power system will be notably reduced, causing in a loss of system inertia. A PV sys- 
tem with Virtual Inertia Emulator system equipped with a HESS was investigated in this chapter to help improve system inertial response.

Control systems for the VIE and DC-DC converters of the HESS are designed which have the following advantages: 1) fast voltage regulation; 2) smoothing the PV output power 3) lowering charge/discharge current rates of battery; 4) reducing current stress levels on battery; 5) improving life span of battery; and 6) enhancing system inertial response. 


\section{CHAPTER 8}

\section{CONCLUSION AND FUTURE WORK}

\subsection{Conclusion}

The integration of renewable energy generators with has been of extreme attention to the researchers and power system operators recently. Universities and other research centers are still performing cutting-edge studies to find and evaluate the opportunities for enhancing the large utilization of renewable energy sources. However, grid integration of large these sources of energy, particularly in the weak grid can cause the undesirable effects on the legacy grid performance in many considerable ways. Some examples of these effects could be frequency and voltage stability, significant harmonics injection, and increasing the number of capacitor bank and voltage regulator operations. To decrease the influence of these threats, this dissertation has developed the advanced methods for renewable energy integration into the grid addressing intermittency, harmonics, and inertial response. In this dissertation, several features related to the control, design, implementation, and energy management of the Microgrid systems with renewable energy sources are presented.

To fulfill the objectives of this dissertation, first, a comprehensive review on challenges and developments in grid-connected photovoltaic systems is provided. Many international and North American organizations such as 1547.8 group and IEEE 1547.7 are defining modeling recommendations and methodologies for renewable energy interconnection. Photovoltaic inverter manufacturers, utilities, and other involved area experts are focused on designing improved smart control strategies for PV inverters. However, there are still numerous gaps in the dynamic analysis of grid-connected PV performance. The increase of large-scale PV-penetration circumstances needs a more thorough investigation of these gaps and systematic interconnection studies. Several technical challenges and required fundamentals for implementation of renewable energies in the grid was provided in 
this study. Secure, safe, and economic proper operation of the power network at voltage and frequency violations for different countries are specified by the grid codes.

Inverters can be considered as the brain of a PV system. The inverter topologies are classified based on the number of power processing steps, the position of capacitors for decoupling the power, if they utilize transformers or not, and kinds of grid interface. Moreover, many control methods have been suggested by researchers to control the MPPT, current, voltage, active and reactive power. An overview of these controllers was discussed in this dissertation.

Higher solar integration requires implementation of battery (and super-capacitor) energy storage systems to compensate high energy (and high power) fluctuations caused by stochastic nature of renewable resources. A brief description of energy storage systems as a whole, problems that are presented with the large-scale integration of PV renewable energy, and the uses of energy storage systems to provide a means to resolve those problems were similarly discussed in this dissertation.

Furthermore, to assess the effects of renewable energies on the reduction of GHG, this dissertation presents an analysis of carbon tax, as strategy option, for encouraging the use of renewable-based DGs and evaluate the cost-effectiveness of the system in terms of GHG mitigation potentials. Different scenarios include different types of RES and different penetration levels of the distributed generation. An economic study for the NPC with both WT and PV+WT was also conducted which shows that, through imposing appropriate taxes on $\mathrm{Co} 2$ and setting remunerative "buy back" prices by the government, there are remarkable potentials of $\mathrm{Co} 2$ mitigation along with $\mathrm{COE}$ reduction. Similarly, technical analysis was accompanied by both HOMER and Matlab Simulink to show that the proposed Renewable energy system can be used in the real world.

In summary, there are enough prospects for renewable-based DG generation in existence of appropriate policy allocation for GHG emission penalties and Market Price 
Referent (MPR), as well as technical measures, in order them to be competitive with the grid and therefore keep away from environmental emissions from the centralized grid. It is suggested that further studies be performed on offshore wind turbines in combination with subsea transmission power supplies.

According to a nonlinear current-voltage characteristic of Photovoltaics (PV) we need to track maximum power output of PV generation units instantly. This study introduces a non-complicated method for tracking the maximum power point without any previous knowledge of the physical parameters linked with a Grid-Connected photovoltaic system using artificial neural networks (ANN) modeling. The productivity of the suggested Artificial Neural Network structures for the MPPT control and the forecast of Duty Cycle of DC-DC boost converter has been presented. Since the duty cycle is directly achieved by using ANN, the proposed system does not need complicated processes and cutting-edge power electronic control units. The results show that the ANN is sufficiently accurate and can identify the duty cycle under different solar irradiance.

In order to analyze the voltage profile and power quality due to grid-tied PV, a comprehensive study was conducted on a 1.1MW PV power plant, tied to a distribution feeder network in Miami, Florida. Power quality monitoring results of the Photovoltaic power plant (as our testbed) installed at Florida International University. Site description and list of components are provided. Base on the analysis, steady state voltage violations have occurred on Jun 20 to Jul 16 (Over-voltages). However, some spikes happen during the period of study, which was not because of PV installation. Based on ITIC curves momentary voltage spikes can cause problems for sensitive electric devices and computers, but not to the grid.Voltage THD Violation only happens on phase and they were below 5.3\%. Voltage THDs may not cause major problems in the Feeder. However, current THD has an inverse relationship with PV power production. When PV production is lower than 451kW, current THDs exceed 5\% limit the IEEE 1547. 
A system model and data from the plant's data acquisition unit and power quality recorder was constructed. Multiple use-cases and scenarios were delineated for the two studies. Power quality issues were studied using high-resolution data for current and voltage THDs based on real measurements. It was concluded that no problematic issues persisted at the existing penetration level of $1.1 \mathrm{MW}$. Current THDs over $5 \%$ has been increased when the power output is less than $451 \mathrm{~kW}$ and it has a tight connection to the output power. Voltage profile analyses for steady-state and time-series scenarios revealed that at $60 \%$ penetration level, significant impacts due to voltage deviation and feeder losses could be observed. Further, the number of switching operations for voltage regulators increases dramatically when PV inverters operate in Volt/VAr control mode, followed by Volt/Watt, and finally Power Factor. Although unity power factor causes the least number of operations, the grid codes require the use of Volt/VAr mode for inverter control. Hence, strategies to mitigate these impacts are required.

In order to reduce the influence of the grid harmonic currents and voltages, harmonic compensation is regularly implemented for a grid-tied inverter. In this dissertation, the principles of the conventional $L L C L$ filter and parameter design of the $L(L C L)_{2}$ filters has been proposed. Since grid-side inductance $\left(L_{21}\right)$ of the $L L C L$ filter is mainly decided by the harmonic currents around double the switching frequency instead of those around the switching frequency, it has been replaced by a small trap at double the switching frequency. Compared to the $L L C L$ filter, the replacement results in a reduction of the total inductance size, and hence, the total loss of the filter. The inverter-side inductance is divided into three parts to place resonant branches in between them. Therefore, the $L(L C L)_{2}$ filter has lower loss and better performance at high-order harmonics attenuation. In the proposed design, the maximum power factor variation remained unchanged and the current THD has improved by $7.77 \%$.

A $700 \mathrm{~W}$ single-phase grid-tied inverter is designed to compare the characteristics 
of the conventional $L L C L$ filter and the suggested $L(L C L)_{2}$ filter through experimental results. In addition, the stability of the closed-loop system has been analyzed. The results validate that total loss and the value of the inductors of the $L(L C L)_{2}$ filter, reduced by a factor of $3.12 \%$ and $22.22 \%$; respectively, compared to that of the $L L C L$ filter.

Finally, a hybrid energy storage system (HESS) including a battery energy storage system (BESS) and a super-capacitor is assessed in this dissertation. This system is designed to accommodate fast solar power fluctuations because of the intermittent nature of irradiance situations. Also, as conventional generators are replaced by high-penetration levels of renewable energies (mostly PV generation), the existing rotating kinetic energy in the power system will be notably reduced, causing a loss of system inertia. A PV system with Virtual Inertia Emulator system equipped with a HESS was investigated in this chapter 7 to help improve system inertial response.

Control systems for the VIE and DC-DC converters of the HESS are designed which have the following advantages: 1) fast voltage regulation; 2) smoothing the PV output power 3) lowering charge/discharge current rates of battery; 4) reducing current stress levels on battery; 5) improving lifespan of battery, and 6) enhancing system inertial response.

\subsection{Future Work}

The topics covered in this dissertation illustrated the new challenges in real-time control and optimal operation of smart grid system with integration of large renewable energy sources. Due to essential needs for understanding, recognizing and solving probable issues and obstacles of future power systems, it recommended to consider the following topics as the future works:

1. There are several interesting future research areas for the study presented in chapter 7. Fog computing applications as one of the most useful techniques for the future 
smart grid development. Virtual energy storing can be proposed to keep supply and demand curve all through peak hours. For this purpose, optimization (realtime and online), the control flow of energy, and prediction will be done using Fog framework. Moreover, Controller optimization for virtual power flow which will operate in both islanded mode and normal mode with any intermittencies of renewable power generation.

2. Considering further expansion of the $L(L C L)_{2}$ filter the proposed filter in chapter 6 can pave the path for the utilization of this filter as an output filter for newly introduced power electronic devices such as solid state transformers (SST). These multilevel converters are a combination of high power semiconductor units, traditional high-frequency transformers and a control mechanism to provide a flexible control for powering the distribution networks.

3. The concept of HESS in chapter 7 focuses on short term fluctuations and includes no long-term power regulation. For longer term variations in PV output, a new control system can be presented which includes the SOC for the battery and supercapacitor. 


\section{BIBLIOGRAPHY}

[1] "Ieee approved draft standard conformance test procedures for equipment interconnecting distributed resources with electric power systems amendment 1," IEEE P1547.1a/D5, December. 2014, pp. 1-19, May 2015.

[2] J. Cotrell, W. Pratt, J. Cotrell, and W. Pratt, "Ten year power plant site plan 2017 2026," Florida Power \& Light Company, Tech. Rep., 2017.

[3] "Ieee draft recommended practice for establishing methods and procedures that provide supplemental support for implementation strategies for expanded use of ieee standard 1547,"' IEEE P1547.8/D8, July 2014, pp. 1-176, June 2014.

[4] H. Athari, M. Niroomand, and M. Ataei, "Review and classification of control systems in grid-tied inverters," Renewable and Sustainable Energy Reviews, vol. 72, pp. 1167 - 1176, 2017. [Online]. Available: http: //www.sciencedirect.com/science/article/pii/S1364032116306839

[5] Z. Tang and B. Akin, "Suppression of dead-time distortion through revised repetitive controller in pmsm drives," IEEE Transactions on Energy Conversion, vol. 32, no. 3, pp. 918-930, Sept 2017.

[6] F. Blaabjerg, R. Teodorescu, M. Liserre, and A. V. Timbus, "Overview of control and grid synchronization for distributed power generation systems," IEEE Transactions on Industrial Electronics, vol. 53, no. 5, pp. 1398-1409, Oct 2006.

[7] A. Mohamed, V. Salehi, and O. Mohammed, "Real-time energy management algorithm for mitigation of pulse loads in hybrid microgrids," Smart Grid, IEEE Transactions on, vol. 3, no. 4, pp. 1911-1922, Dec 2012.

[8] A. Anzalchi, M. Moghaddami, A. Moghadasi, M. M. Pour, and A. Sarwat, "Design and analysis of a higher order power filter for grid-connected renewable energy systems," IEEE Transactions on Industry Applications, vol. PP, no. 99, pp. 1-1, 2017.

[9] A. Anzalchi, M. Moghaddami, A. Moghaddasi, A. I. Sarwat, and A. K. Rathore, "A new topology of higher order power filter for single-phase grid-tied voltagesource inverters," IEEE Transactions on Industrial Electronics, vol. 63, no. 12, pp. 7511-7522, Dec 2016.

[10] C. SANKARAN, Power Quality. Library of Congress Cataloging-in-Publication Data: CRC PRESS, 2002. 
[11] A. Anzalchi and A.Sarwat, "Artificial neural network based duty cycle estimation for maximum power point tracking in photovoltaic systems," in SoutheastCon 2015, April 2015, pp. 1-5.

[12] N. Harischandrappa and A. K. S. Bhat, "A fixed-frequency lcl -type series resonant converter with a capacitive output filter using a modified gating scheme," IEEE Transactions on Industry Applications, vol. 50, no. 6, pp. 4056-4064, Nov 2014.

[13] M. Lindgren and J. Svensson, "Connecting fast switching voltage source converters to the grid-harmonic distortion and its reduction," in Stockholm Power Tech. Conf., June 18-22 1995, pp. 191-195.

[14] M. Liserre, F. Blaabjerg, and S. Hansen, "Design and control of an lcl-filter-based three-phase active rectifier," Industry Applications, IEEE Transactions on, vol. 41, no. 5, pp. 1281-1291, Sept 2005.

[15] I. Gabe, V. Montagner, and H. Pinheiro, "Design and implementation of a robust current controller for vsi connected to the grid through an lcl filter," Power Electronics, IEEE Transactions on, vol. 24, no. 6, pp. 1444-1452, June 2009.

[16] A. Reznik, M. G. Sim?es, A. Al-Durra, and S. M. Muyeen, "Lcl filter design and performance analysis for grid-interconnected systems," IEEE Transactions on Industry Applications, vol. 50, no. 2, pp. 1225-1232, March 2014.

[17] A. Houari, H. Renaudineau, J. P. Martin, B. Nahid-Mobarakeh, S. Pierfederici, and F. Meibody-Tabar, "Large-signal stabilization of ac grid supplying voltage-source converters with lcl-filters," IEEE Transactions on Industry Applications, vol. 51, no. 1, pp. 702-711, Jan 2015.

[18] A. Khodaei, "Resiliency-oriented microgrid optimal scheduling," IEEE Transactions on Smart Grid, vol. 5, no. 4, pp. 1584-1591, July 2014.

[19] Y. V. P. Kumar and R. Bhimasingu, "Improving resiliency in renewable energy based green microgrids using virtual synchronous machines controlled inverter," in 2015 IEEE Innovative Smart Grid Technologies - Asia (ISGT ASIA), Nov 2015, pp. 1-6.

[20] Y. Zheng, "Virtual inertia emulation in islanded microgrids with energy storage system," Master's thesis. 
[22] W. Wu, Y. He, T. Tang, and F. Blaabjerg, "A new design method for the passive damped lcl and llcl filter-based single-phase grid-tied inverter," Industrial Electronics, IEEE Transactions on, vol. 60, no. 10, pp. 4339-4350, Oct 2013.

[23] F. Garcia, A. Ferreira, and J. Pomilio, "Control strategy for battery-ultracapacitor hybrid energy storage system," in Applied Power Electronics Conference and Exposition, 2009. APEC 2009. Twenty-Fourth Annual IEEE, Feb 2009, pp. 826-832.

[24] H. Bevrani, T. Ise, and Y. Miura, "Virtual synchronous generators: A survey and new perspectives," International Journal of Electrical Power and Energy Systems, vol. 54, pp. 244 - 254, 2014. [Online]. Available: http://www.sciencedirect.com/science/article/pii/S0142061513003062

[25] Y. Chen, R. Hesse, D. Turschner, and H. P. Beck, "Improving the grid power quality using virtual synchronous machines," in 2011 International Conference on Power Engineering, Energy and Electrical Drives, May 2011, pp. 1-6.

[26] A. Anzalchi and A. Sarwat, "Analysis of carbon tax as an incentive toward building sustainable grid with renewable energy utilization," in Green Technologies Conference (GreenTech), 2015 Seventh Annual IEEE, April 2015, pp. 103-109.

[27] M. Rahman and A. Oo, "Distributed multi-agent based coordinated power management and control strategy for microgrids with distributed energy resources," Energy Conversion and Management, vol. 139, pp. 20 32, 2017. [Online]. Available: http://www.sciencedirect.com/science/article/ pii/S0196890417301255

[28] P. Ifaei, A. Karbassi, G. Jacome, and C. Yoo, "A systematic approach of bottom-up assessment methodology for an optimal design of hybrid solar/wind energy resources case study at middle east region," Energy Conversion and Management, vol. 145, pp. 138 - 157, 2017. [Online]. Available: http://www.sciencedirect.com/science/article/pii/S019689041730417X

[29] B. Ye, K. Zhang, J. Jiang, L. Miao, and J. Li, "Towards a 90future: A case study of an island in the south china sea," Energy Conversion and Management, vol. 142, pp. 28 - 41, 2017. [Online]. Available: http://www.sciencedirect.com/science/article/pii/S0196890417302522

[30] A. Cabrera-Tobar, E. Bullich-Massagu, M. Arags-Pealba, and O. GomisBellmunt, "Review of advanced grid requirements for the integration of large scale photovoltaic power plants in the transmission system," Renewable and 
Sustainable Energy Reviews, vol. 62, pp. 971 - 987, 2016. [Online]. Available: http://www.sciencedirect.com/science/article/pii/S136403211630154X

[31] A. S. Mundada, Y. Nilsiam, and J. M. Pearce, "A review of technical requirements for plug-and-play solar photovoltaic microinverter systems in the united states," Solar Energy, vol. 135, pp. 455 - 470, 2016. [Online]. Available: http://www.sciencedirect.com/science/article/pii/S0038092X16301815

[32] M. A. Eltawil and Z. Zhao, "Grid-connected photovoltaic power systems: Technical and potential problemsa review," Renewable and Sustainable Energy Reviews, vol. 14, no. 1, pp. 112 - 129, 2010. [Online]. Available: http://www.sciencedirect.com/science/article/pii/S1364032109001749

[33] J. Jana, H. Saha, and K. D. Bhattacharya, "A review of inverter topologies for single-phase grid-connected photovoltaic systems," Renewable and Sustainable Energy Reviews, vol. 72, pp. 1256 - 1270, 2017. [Online]. Available: http://www.sciencedirect.com/science/article/pii/S1364032116306943

[34] H. de Faria, F. B. Trigoso, and J. A. Cavalcanti, "Review of distributed generation with photovoltaic grid connected systems in brazil: Challenges and prospects," Renewable and Sustainable Energy Reviews, vol. 75, pp. 469 - 475, 2017. [Online]. Available: http://www.sciencedirect.com/science/article/pii/S1364032116307407

[35] N. Sommerfeldt and H. Madani, "Revisiting the techno-economic analysis process for building-mounted, grid-connected solar photovoltaic systems: Part one review," Renewable and Sustainable Energy Reviews, vol. 74, pp. 1379 1393, 2017. [Online]. Available: http://www.sciencedirect.com/science/article/pii/ S1364032116310048

[36] C. Lupangu and R. Bansal, "A review of technical issues on the development of solar photovoltaic systems," Renewable and Sustainable Energy Reviews, vol. 73, pp. 950 - 965, 2017. [Online]. Available: http://www.sciencedirect.com/science/ article/pii/S1364032117302022

[37] W. Xiao, M. S. E. Moursi, O. Khan, and D. Infield, "Review of grid-tied converter topologies used in photovoltaic systems," IET Renewable Power Generation, vol. 10, no. 10, pp. 1543-1551, 2016.

[38] Y. K. Wu, J. H. Lin, and H. J. Lin, "Standards and guidelines for grid-connected photovoltaic generation systems: A review and comparison," IEEE Transactions on Industry Applications, vol. 53, no. 4, pp. 3205-3216, July 2017. 
[39] S. C. Smith, P. K. Sen, B. Kroposki, and K. Malmedal, "Renewable energy and energy storage systems in rural electrical power systems: Issues, challenges and application guidelines," in 2010 IEEE Rural Electric Power Conference (REPC), May 2010, pp. B4-B4-7.

[40] S. D. G. Jayasinghe, D. M. Vilathgamuwa, and U. K. Madawala, "Dual inverter based battery energy storage system for grid connected photovoltaic systems," in IECON 2010 - 36th Annual Conference on IEEE Industrial Electronics Society, Nov 2010, pp. 3275-3280.

[41] V. Fthenakis, "Sustainability of photovoltaics: The case for thin-film solar cells," Renewable and Sustainable Energy Reviews, vol. 13, no. 9, pp. 2746 2750, 2009. [Online]. Available: http://www.sciencedirect.com/science/article/pii/ S1364032109000896

[42] Y. P. Agalgaonkar and D. J. Hammerstrom, "Evaluation of smart grid technologies employed for system reliability improvement: Pacific northwest smart grid demonstration experience," IEEE Power and Energy Technology Systems Journal, vol. PP, no. 99, pp. 1-1, 2017.

[43] S. Lakshminarayana, T. Q. S. Quek, and H. V. Poor, "Combining cooperation and storage for the integration of renewable energy in smart grids," in 2014 IEEE Conference on Computer Communications Workshops (INFOCOM WKSHPS), April 2014, pp. 622-627.

[44] Y. Tang and R. Ayyanar, "Methodology of automated protection analysis for large distribution feeders with high penetration of photovoltaic systems," IEEE Power and Energy Technology Systems Journal, vol. 4, no. 1, pp. 1-9, March 2017.

[45] R. K. Varma, S. A. Rahman, V. Atodaria, S. Mohan, and T. Vanderheide, "Technique for fast detection of short circuit current in pv distributed generator," IEEE Power and Energy Technology Systems Journal, vol. 3, no. 4, pp. 155-165, Dec 2016.

[46] S. Bu, F. R. Yu, P. X. Liu, and P. Zhang, "Distributed scheduling in smart grid communications with dynamic power demands and intermittent renewable energy resources," in 2011 IEEE International Conference on Communications Workshops (ICC), June 2011, pp. 1-5.

[47] O. M. Toledo, D. O. Filho, and A. S. A. C. Diniz, "Distributed photovoltaic generation and energy storage systems: A review," Renewable and Sustainable 
Energy Reviews, vol. 14, no. 1, pp. 506 - 511, 2010. [Online]. Available: http://www.sciencedirect.com/science/article/pii/S136403210900207X

[48] H. Ibrahim, A. Ilinca, and J. Perron, "Energy storage systemscharacteristics and comparisons," Renewable and Sustainable Energy Reviews, vol. 12, no. 5, pp. 1221 - 1250, 2008. [Online]. Available: http://www.sciencedirect.com/science/ article/pii/S1364032107000238

[49] T. Nikolakakis and V. Fthenakis, "The optimum mix of electricity from windand solar-sources in conventional power systems: Evaluating the case for new york state," Energy Policy, vol. 39, no. 11, pp. 6972 - 6980, 2011, asian Energy Security. [Online]. Available: http://www.sciencedirect.com/science/article/pii/ S0301421511004526

[50] N. H. Afgan, D. A. Gobaisi, M. G. Carvalho, and M. Cumo, "Sustainable energy development," Renewable and Sustainable Energy Reviews, vol. 2, no. 3, pp. 235 - 286, 1998. [Online]. Available: http://www.sciencedirect.com/science/article/ pii/S1364032198000021

[51] B. Gohla-Neudecker, U. Wagner, and T. Hamacher, "Analysis of renewable energy grid integration by range extension technologies of bevs," in 2013 International Conference on Clean Electrical Power (ICCEP), June 2013, pp. 155-162.

[52] P. Denholm and R. M. Margolis, "Evaluating the limits of solar photovoltaics (pv) in electric power systems utilizing energy storage and other enabling technologies," Energy Policy, vol. 35, no. 9, pp. 4424 - 4433, 2007. [Online]. Available: http://www.sciencedirect.com/science/article/pii/S030142150700095X

[53] N. Papanikolaou, A. Kyritsis, M. Loupis, C. Tzotzos, and E. Zoga, "Design considerations for single-phase line frequency transformers applied at photovoltaic systems," IEEE Power and Energy Technology Systems Journal, vol. 2, no. 3, pp. 82-93, Sept 2015.

[54] B. Guha, R. J. Haddad, and Y. Kalaani, "Voltage ripple-based passive islanding detection technique for grid-connected photovoltaic inverters," IEEE Power and Energy Technology Systems Journal, vol. 3, no. 4, pp. 143-154, Dec 2016.

[55] S. B. Kjaer, J. K. Pedersen, and F. Blaabjerg, "A review of single-phase gridconnected inverters for photovoltaic modules," IEEE Transactions on Industry Applications, vol. 41, no. 5, pp. 1292-1306, Sept 2005. 
[56] M. Meinhardt and G. Cramer, "Past, present and future of grid connected photovoltaic- and hybrid-power-systems," in 2000 Power Engineering Society Summer Meeting (Cat. No.00CH37134), vol. 2, 2000, pp. 1283-1288 vol. 2.

[57] M. Nagao and K. Harada, "Power flow of photovoltaic system using buck-boost pwm power inverter," in Proceedings of Second International Conference on Power Electronics and Drive Systems, vol. 1, May 1997, pp. 144-149 vol.1.

[58] M. A. Moonem and H. Krishnaswami, "Control and configuration of three-level dual-active bridge dc-dc converter as a front-end interface for photovoltaic system," in 2014 IEEE Applied Power Electronics Conference and Exposition - APEC 2014, March 2014, pp. 3017-3020.

[59] B.-R. Lin and T.-Y. Yang, "Implementation of active power filter with asymmetrical inverter legs for harmonic and reactive power compensation," Electric Power Systems Research, vol. 73, no. 2, pp. 227 - 237, 2005. [Online]. Available: http://www.sciencedirect.com/science/article/pii/S0378779604001828

[60] H. Yamamoto, C. Y. Inaba, E. Hiraki, and M. Nakaoka, "Multifunctional digitallycontrolled bidirectional three-phase soft-switching pwm converter," in 2000 Eighth International Conference on Power Electronics and Variable Speed Drives (IEE Conf. Publ. No. 475), 2000, pp. 86-90.

[61] S. Chandhaket, A. Yoshida, H. Eiji, M. Nakamura, Y. Konishi, and M. Nakaoka, "Multi-functional digitally-controlled bidirectional interactive three-phase softswitching pwm converter with resonant snubbers," in 2001 IEEE 32nd Annual Power Electronics Specialists Conference (IEEE Cat. No.01CH37230), vol. 2, 2001, pp. 589-593 vol.2.

[62] A. Anzalchi, M. Moghaddami, A. Moghaddasi, M. M. Pour, and A. Sarwat, "A modified higher order power filter for grid-connected renewable energy systems," in 2016 IEEE/IAS 52nd Industrial and Commercial Power Systems Technical Conference (I\&CPS), May 2016, pp. 1-9.

[63] N. D. Tuyen and G. Fujita, "Pv-active power filter combination supplies power to nonlinear load and compensates utility current," IEEE Power and Energy Technology Systems Journal, vol. 2, no. 1, pp. 32-42, March 2015.

[64] L. Liu, H. Li, and Y. Xue, "A coordinated active and reactive power control strategy for grid-connected cascaded photovoltaic (pv) system in high voltage high power applications," in 2013 Twenty-Eighth Annual IEEE Applied Power Electronics Conference and Exposition (APEC), March 2013, pp. 1301-1308. 
[65] E. Babaei and S. H. Hosseini, "New cascaded multilevel inverter topology with minimum number of switches," Energy Conversion and Management, vol. 50, no. 11, pp. 2761 - 2767, 2009. [Online]. Available: http: //www.sciencedirect.com/science/article/pii/S0196890409002489

[66] I. Colak, E. Kabalci, and R. Bayindir, "Review of multilevel voltage source inverter topologies and control schemes," Energy Conversion and Management, vol. 52, no. 2, pp. 1114 - 1128, 2011. [Online]. Available: http://www.sciencedirect.com/science/article/pii/S0196890410004085

[67] S. W. Mohod and M. V. Aware, "Micro wind power generator with battery energy storage for critical load," IEEE Systems Journal, vol. 6, no. 1, pp. 118-125, March 2012.

[68] M. I. Marei, E. F. El-Saadany, and M. M. A. Salama, "A novel control algorithm for the dg interface to mitigate power quality problems," IEEE Transactions on Power Delivery, vol. 19, no. 3, pp. 1384-1392, July 2004.

[69] P. T. Cheng, C. A. Chen, T. L. Lee, and S. Y. Kuo, "A cooperative imbalance compensation method for distributed-generation interface converters," IEEE Transactions on Industry Applications, vol. 45, no. 2, pp. 805-815, March 2009.

[70] Z. Lv, A. Luo, C. Wu, Z. Shuai, and Z. Kang, "A research of microgrid energy supply and filtering system based on inverter multiplexing," in 2009 International Conference on Sustainable Power Generation and Supply, April 2009, pp. 1-7.

[71] B. Han, B. Bae, H. Kim, and S. Baek, "Combined operation of unified powerquality conditioner with distributed generation," IEEE Transactions on Power Delivery, vol. 21, no. 1, pp. 330-338, Jan 2006.

[72] Y. Li, D. M. Vilathgamuwa, and P. C. Loh, "Microgrid power quality enhancement using a three-phase four-wire grid-interfacing compensator," IEEE Transactions on Industry Applications, vol. 41, no. 6, pp. 1707-1719, Nov 2005.

[73] F. Wang, J. L. Duarte, and M. A. M. Hendrix, "Grid-interfacing converter systems with enhanced voltage quality for microgrid application - concept and implementation," IEEE Transactions on Power Electronics, vol. 26, no. 12, pp. 3501-3513, Dec 2011.

[74] X. Yu and A. M. Khambadkone, "Multi-functional power converter building block to facilitate the connection of micro-grid," in 2008 11th Workshop on Control and Modeling for Power Electronics, Aug 2008, pp. 1-6. 
[75] S. Dasgupta, S. N. Mohan, S. K. Sahoo, and S. K. Panda, "A lyapunov function based current controller to control active and reactive power flow in a three phase grid connected pv inverter under generalized grid voltage conditions," in 8th International Conference on Power Electronics - ECCE Asia, May 2011, pp. 11101117.

[76] L. Cheng, R. Cheung, and K. H. Leung, "Advanced photovoltaic inverter with additional active power line conditioning capability," in PESC97. Record 28th Annual IEEE Power Electronics Specialists Conference. Formerly Power Conditioning Specialists Conference 1970-71. Power Processing and Electronic Specialists Conference 1972, vol. 1, Jun 1997, pp. 279-283 vol.1.

[77] S. Naderi, E. Pouresmaeil, and W. D. Gao, "The frequency-independent control method for distributed generation systems," Applied Energy, vol. 96, pp. 272 - 280, 2012, smart Grids. [Online]. Available: http://www.sciencedirect.com/ science/article/pii/S0306261911006374

[78] R. R. Sawant and M. C. Chandorkar, "A multifunctional four-leg grid-connected compensator," IEEE Transactions on Industry Applications, vol. 45, no. 1, pp. 249259, Jan 2009.

[79] F. Wang, J. L. Duarte, and M. A. M. Hendrix, "Control of grid-interfacing inverters with integrated voltage unbalance correction," in 2008 IEEE Power Electronics Specialists Conference, June 2008, pp. 310-316.

[80] R. Majumder, A. Ghosh, G. Ledwich, and F. Zare, "Load sharing and power quality enhanced operation of a distributed microgrid," IET Renewable Power Generation, vol. 3, no. 2, pp. 109-119, June 2009.

[81] C. J. Gajanayake, D. M. Vilathgamuwa, P. C. Loh, R. Teodorescu, and F. Blaabjerg, "Z-source-inverter-based flexible distributed generation system solution for grid power quality improvement," IEEE Transactions on Energy Conversion, vol. 24, no. 3, pp. 695-704, Sept 2009.

[82] G. Tsengenes and G. Adamidis, "A multi-function grid connected pv system with three level npc inverter and voltage oriented control," Solar Energy, vol. 85, no. 11, pp. 2595 - 2610, 2011. [Online]. Available: http://www.sciencedirect.com/science/article/pii/S0038092X11002593

[83] M. Saitou and T. Shimizu, "Generalized theory of instantaneous active and reactive powers in single-phase circuits based on hilbert transform," in 2002 IEEE 
33rd Annual IEEE Power Electronics Specialists Conference. Proceedings (Cat. No.02CH37289), vol. 3, 2002, pp. 1419-1424 vol.3.

[84] M. T. Abolhassani, P. Enjeti, and H. Toliyat, "Integrated doubly fed electric alternator/active filter (idea), a viable power quality solution, for wind energy conversion systems," IEEE Transactions on Energy Conversion, vol. 23, no. 2, pp. 642-650, June 2008.

[85] T. F. Wu, C. L. Shen, C. H. Chang, and J. Y. Chiu, "A 1 phi;3w grid-connection pv power inverter with partial active power filter," in 2002 IEEE 33rd Annual IEEE Power Electronics Specialists Conference. Proceedings (Cat. No.02CH37289), vol. 3, 2002, pp. 1512-1517 vol.3.

[86] J. He, M. S. Munir, and Y. W. Li, "Opportunities for power quality improvement through dg-grid interfacing converters," in The 2010 International Power Electronics Conference - ECCE ASIA -, June 2010, pp. 1657-1664.

[87] H. Yu, J. Pan, and A. Xiang, "A multi-function grid-connected pv system with reactive power compensation for the grid," Solar Energy, vol. 79, no. 1, pp. 101 106, 2005. [Online]. Available: http://www.sciencedirect.com/science/article/pii/ S0038092X04003019

[88] S. Kim, G. Yoo, and J. Song, "A bifunctional utility connected photovoltaic system with power factor correction and ups facility," in Conference Record of the Twenty Fifth IEEE Photovoltaic Specialists Conference - 1996, May 1996, pp. 1363-1368.

[89] R. I. Bojoi, L. R. Limongi, D. Roiu, and A. Tenconi, "Enhanced power quality control strategy for single-phase inverters in distributed generation systems," IEEE Transactions on Power Electronics, vol. 26, no. 3, pp. 798-806, March 2011.

[90] M. Cirrincione, M. Pucci, and G. Vitale, "A single-phase dg generation unit with shunt active power filter capability by adaptive neural filtering," IEEE Transactions on Industrial Electronics, vol. 55, no. 5, pp. 2093-2110, May 2008.

[91] K. J. P. Macken, K. Vanthournout, J. V. den Keybus, G. Deconinck, and R. J. M. Belmans, "Distributed control of renewable generation units with integrated active filter," IEEE Transactions on Power Electronics, vol. 19, no. 5, pp. 1353-1360, Sept 2004.

[92] S. H. Hosseini, S. Danyali, and A. Y. Goharrizi, "Single stage single phase seriesgrid connected pv system for voltage compensation and power supply," in 2009 IEEE Power Energy Society General Meeting, July 2009, pp. 1-7. 
[93] S. Dasgupta, S. K. Sahoo, S. K. Panda, and G. A. J. Amaratunga, "Single-phase inverter-control techniques for interfacing renewable energy sources with microgrid - part ii: Series-connected inverter topology to mitigate voltage-related problems along with active power flow control," IEEE Transactions on Power Electronics, vol. 26, no. 3, pp. 732-746, March 2011.

[94] B.-R. Lin and T.-Y. Yang, "Active power filter with asymmetrical inverter legs for harmonic and reactive power compensation," in Circuits and Systems, 2004. MWSCAS '04. The 2004 47th Midwest Symposium on, vol. 2, July 2004, pp. II285-II-288 vol.2.

[95] R. D. Patidar, S. P. Singh, and D. K. Khatod, "Single-phase single-stage gridinteractive photovoltaic system with active filter functions," in IEEE PES General Meeting, July 2010, pp. 1-7.

[96] K. Hirachi, T. Mii, T. Nakashiba, K. G. D. Laknath, and M. Nakaoka, "Utilityinteractive multi-functional bidirectional converter for solar photovoltaic power conditioner with energy storage batteries," in Proceedings of the 1996 IEEE IECON. 22nd International Conference on Industrial Electronics, Control, and Instrumentation, vol. 3, Aug 1996, pp. 1693-1698 vol.3.

[97] S. Dasgupta, S. K. Sahoo, and S. K. Panda, "Single-phase inverter control techniques for interfacing renewable energy sources with microgrid - part i: Parallelconnected inverter topology with active and reactive power flow control along with grid current shaping," IEEE Transactions on Power Electronics, vol. 26, no. 3, pp. 717-731, March 2011.

[98] H. R. Seo, S. J. Jang, G. H. Kim, M. Park, and I. K. Yu, "Hardware based performance analysis of a multi-function single-phase pv-af system," in 2009 IEEE Energy Conversion Congress and Exposition, Sept 2009, pp. 2213-2217.

[99] Y. E. Wu and C. L. Shen, "Implementation of a dc power system with pv gridconnection and active power filtering," in The 2nd International Symposium on Power Electronics for Distributed Generation Systems, June 2010, pp. 116-121.

[100] T. F. Wu, H. S. Nien, H. M. Hsieh, and C. L. Shen, "Pv power injection and active power filtering with amplitude-clamping and amplitude-scaling algorithms," IEEE Transactions on Industry Applications, vol. 43, no. 3, pp. 731-741, May 2007.

[101] T. F. Wu, C. H. Chang, and Y. K. Chen, "A multi-function photovoltaic power supply system with grid-connection and power factor correction features," in 2000 
IEEE 31st Annual Power Electronics Specialists Conference. Conference Proceedings (Cat. No.00CH37018), vol. 3, 2000, pp. 1185-1190 vol.3.

[102] S. Sladi, B. Barii, and M. Sokovi, "Cost-effective power converter for thin film solar cell technology and improved power quality," Journal of Materials Processing Technology, vol. 201, no. 13, pp. 786 - 790, 2008, 10th International Conference on Advances in Materials and Processing TechnologiesAMPT 2007. [Online]. Available: http://www.sciencedirect.com/ science/article/pii/S0924013607013052

[103] S. J. Chiang, K. T. Chang, and C. Y. Yen, "Residential photovoltaic energy storage system," IEEE Transactions on Industrial Electronics, vol. 45, no. 3, pp. 385-394, Jun 1998.

[104] Y.-C. Kuo, T.-J. Liang, and J.-F. Chen, "A high-efficiency single-phase three-wire photovoltaic energy conversion system," IEEE Transactions on Industrial Electronics, vol. 50, no. 1, pp. 116-122, Feb 2003.

[105] A. Chaitanakulwat, V. Kinnares, and N. Thungsuk, "Single-phase grid-connected photovoltaic system with active power filter functionality," in 2012 15th International Conference on Electrical Machines and Systems (ICEMS), Oct 2012, pp. $1-3$.

[106] H. Calleja and H. Jimenez, "Performance of a grid connected $\{P V\}$ system used as active filter," Energy Conversion and Management, vol. 45, no. 1516, pp. 2417 - 2428, 2004. [Online]. Available: http://www.sciencedirect.com/science/article/ pii/S0196890403003613

[107] R. A. Mastromauro, M. Liserre, and A. Dell'Aquila, "Pv system power quality enhancement by means of a voltage controlled shunt-converter," in 2008 IEEE Power Electronics Specialists Conference, June 2008, pp. 2358-2363.

[108] Y.-C. Kuo, T.-J. Liang, and J.-F. Chen, "Novel maximum-power-point-tracking controller for photovoltaic energy conversion system," IEEE Transactions on Industrial Electronics, vol. 48, no. 3, pp. 594-601, Jun 2001.

[109] T. F. Wu, H. S. Nei, C. L. Shen, and G. F. Li, "A single-phase two-wire gridconnection pv inverter with active power filtering and nonlinear inductance consideration," in Applied Power Electronics Conference and Exposition, 2004. APEC '04. Nineteenth Annual IEEE, vol. 3, 2004, pp. 1566-1571 Vol.3. 
[110] N. Altin and S. Ozdemir, "Three-phase three-level grid interactive inverter with fuzzy logic based maximum power point tracking controller," Energy Conversion and Management, vol. 69, pp. 17 - 26, 2013. [Online]. Available: http://www.sciencedirect.com/science/article/pii/S0196890413000320

[111] N. Altin and brahim Sefa, "dspace based adaptive neuro-fuzzy controller of grid interactive inverter," Energy Conversion and Management, vol. 56, pp. 130 139, 2012. [Online]. Available: http://www.sciencedirect.com/science/article/pii/ S0196890411003359

[112] J. P. Ram, T. S. Babu, and N. Rajasekar, "A comprehensive review on solar $\{\mathrm{PV}\}$ maximum power point tracking techniques," Renewable and Sustainable Energy Reviews, vol. 67, pp. 826 - 847, 2017. [Online]. Available: http://www.sciencedirect.com/science/article/pii/S1364032116305706

[113] G. Mokhtari, A. Ghosh, G. Nourbakhsh, and G. Ledwich, "Smart robust resources control in lv network to deal with voltage rise issue," IEEE Transactions on Sustainable Energy, vol. 4, no. 4, pp. 1043-1050, Oct 2013.

[114] K. Turitsyn, P. Sulc, S. Backhaus, and M. Chertkov, "Local control of reactive power by distributed photovoltaic generators," in 2010 First IEEE International Conference on Smart Grid Communications, Oct 2010, pp. 79-84.

[115] R. Tonkoski, L. A. C. Lopes, and T. H. M. El-Fouly, "Coordinated active power curtailment of grid connected pv inverters for overvoltage prevention," IEEE Transactions on Sustainable Energy, vol. 2, no. 2, pp. 139-147, April 2011.

[116] E. Demirok, D. Sera, R. Teodorescu, P. Rodriguez, and U. Borup, "Evaluation of the voltage support strategies for the low voltage grid connected pv generators," in 2010 IEEE Energy Conversion Congress and Exposition, Sept 2010, pp. 710-717.

[117] E. Demirok, P. C. Gonzlez, K. H. B. Frederiksen, D. Sera, P. Rodriguez, and R. Teodorescu, "Local reactive power control methods for overvoltage prevention of distributed solar inverters in low-voltage grids," IEEE Journal of Photovoltaics, vol. 1, no. 2, pp. 174-182, Oct 2011.

[118] M. Braun, T. Stetz, R. Brndlinger, C. Mayr, K. Ogimoto, H. Hatta, H. Kobayashi, B. Kroposki, B. Mather, M. Coddington, K. Lynn, G. Graditi, A. Woyte, and I. MacGill, "Is the distribution grid ready to accept large-scale photovoltaic deployment? state of the art, progress, and future prospects," Progress in Photovoltaics: Research and Applications, vol. 20, no. 6, pp. 681-697, 2012. [Online]. Available: http://dx.doi.org/10.1002/pip.1204 
[119] R. Tonkoski, L. A. C. Lopes, and T. H. M. EL-Fouly, "Droop-based active power curtailment for overvoltage prevention in grid connected pv inverters," in 2010 IEEE International Symposium on Industrial Electronics, July 2010, pp. 23882393.

[120] K. Tanaka, M. Oshiro, S. Toma, A. Yona, T. Senjyu, T. Funabashi, and C. H. Kim, "Decentralised control of voltage in distribution systems by distributed generators," IET Generation, Transmission Distribution, vol. 4, no. 11, pp. 1251-1260, November 2010.

[121] S. Weckx, C. Gonzalez, and J. Driesen, "Combined central and local active and reactive power control of pv inverters," IEEE Transactions on Sustainable Energy, vol. 5, no. 3, pp. 776-784, July 2014.

[122] L. Liu, H. Li, Y. Xue, and W. Liu, "Decoupled active and reactive power control for large-scale grid-connected photovoltaic systems using cascaded modular multilevel converters," IEEE Transactions on Power Electronics, vol. 30, no. 1, pp. 176-187, Jan 2015.

[123] P. Monica and M. Kowsalya, "Control strategies of parallel operated inverters in renewable energy application: A review," Renewable and Sustainable Energy Reviews, vol. 65, pp. 885 - 901, 2016. [Online]. Available: http://www.sciencedirect.com/science/article/pii/S1364032116303057

[124] S. Ozdemir, "Z-source t-type inverter for renewable energy systems with proportional resonant controller," International Journal of Hydrogen Energy, vol. 41, no. 29, pp. 12591 - 12602, 2016, special Issue on 3rd European Conference on Renewable Energy Systems (ECRES2015), 710 October 2015, Kemer, Antalya, Turkey. [Online]. Available: http://www.sciencedirect.com/ science/article/pii/S0360319915316360

[125] R. Dufo-Lpez, L. A. Fernndez-Jimnez, I. J. Ramrez-Rosado, J. S. Artal-Sevil, J. A. Domnguez-Navarro, and J. L. Bernal-Agustn, "Daily operation optimisation of hybrid stand-alone system by model predictive control considering ageing model," Energy Conversion and Management, vol. 134, pp. 167 - 177, 2017. [Online]. Available: http://www.sciencedirect.com/science/article/pii/S0196890416311220

[126] G. A. Ramos, R. Costa-Castelló, and J. M. Olm, Repetitive Control. Berlin, Heidelberg: Springer Berlin Heidelberg, 2013, pp. 5-12. [Online]. Available: https://doi.org/10.1007/978-3-642-37778-5_2 
[127] A. Anzalchi, M. M. Pour, and A. Sarwat, "A combinatorial approach for addressing intermittency and providing inertial response in a grid-connected photovoltaic system," in 2016 IEEE Power and Energy Society General Meeting (PESGM), July 2016, pp. 1-5.

[128] M. D. Al-falahi, S. Jayasinghe, and H. Enshaei, "A review on recent size optimization methodologies for standalone solar and wind hybrid renewable energy system," Energy Conversion and Management, vol. 143, pp. 252 274, 2017. [Online]. Available: http://www.sciencedirect.com/science/article/pii/ S0196890417303230

[129] A. Derrouazin, M. Aillerie, N. Mekkakia-Maaza, and J.-P. Charles, "Multi inputoutput fuzzy logic smart controller for a residential hybrid solar-wind-storage energy system," Energy Conversion and Management, vol. 148, pp. 238 250, 2017. [Online]. Available: http://www.sciencedirect.com/science/article/pii/ S0196890417304958

[130] Z. Wu, X. P. Zhang, J. Brandt, S. Y. Zhou, and J. N. LI, “Three control approaches for optimized energy flow with home energy management system," IEEE Power and Energy Technology Systems Journal, vol. 2, no. 1, pp. 21-31, March 2015.

[131] A. Castillo and D. F. Gayme, "Grid-scale energy storage applications in renewable energy integration: A survey," Energy Conversion and Management, vol. 87, pp. 885 - 894, 2014. [Online]. Available: http://www.sciencedirect.com/science/ article/pii/S0196890414007018

[132] Y. Iwafune, T. Ikegami, J. G. da Silva Fonseca, T. Oozeki, and K. Ogimoto, "Cooperative home energy management using batteries for a photovoltaic system considering the diversity of households," Energy Conversion and Management, vol. 96, pp. 322 - 329, 2015. [Online]. Available: http: //www.sciencedirect.com/science/article/pii/S0196890415002046

[133] J. Vandewalle, K. Bruninx, and W. Dhaeseleer, "Effects of large-scale power to gas conversion on the power, gas and carbon sectors and their interactions," Energy Conversion and Management, vol. 94, pp. 28 - 39, 2015. [Online]. Available: http://www.sciencedirect.com/science/article/pii/S0196890415000424

[134] R. Dufo-Lpez and J. L. Bernal-Agustn, "Techno-economic analysis of gridconnected battery storage," Energy Conversion and Management, vol. 91, pp. 394 - 404, 2015. [Online]. Available: http://www.sciencedirect.com/science/article/ pii/S0196890414010772 
[135] R. Amirante, E. Cassone, E. Distaso, and P. Tamburrano, "Overview on recent developments in energy storage: Mechanical, electrochemical and hydrogen technologies," Energy Conversion and Management, vol. 132, pp. 372 387, 2017. [Online]. Available: http://www.sciencedirect.com/science/article/pii/ S019689041631055X

[136] L. Oliveira, M. Messagie, J. Mertens, H. Laget, T. Coosemans, and J. V. Mierlo, "Environmental performance of electricity storage systems for grid applications, a life cycle approach," Energy Conversion and Management, vol. 101, pp. 326 - 335, 2015. [Online]. Available: http: //www.sciencedirect.com/science/article/pii/S0196890415005282

[137] W. Ji, Y. Zhou, Y. Sun, W. Zhang, B. An, and J. Wang, "Thermodynamic analysis of a novel hybrid wind-solar-compressed air energy storage system," Energy Conversion and Management, vol. 142, pp. 176 - 187, 2017. [Online]. Available: http://www.sciencedirect.com/science/article/pii/S019689041730153X

[138] A. N. Ghalelou, A. P. Fakhri, S. Nojavan, M. Majidi, and H. Hatami, "A stochastic self-scheduling program for compressed air energy storage (caes) of renewable energy sources (ress) based on a demand response mechanism," Energy Conversion and Management, vol. 120, pp. 388 - 396, 2016. [Online]. Available: http://www.sciencedirect.com/science/article/pii/S0196890416303399

[139] S. Amodeo, H. Chiacchiarini, J. Solsona, and C. Busada, "High-performance sensorless nonlinear power control of a flywheel energy storage system," Energy Conversion and Management, vol. 50, no. 7, pp. 1722 - 1729, 2009. [Online]. Available: http://www.sciencedirect.com/science/article/pii/S0196890409000983

[140] S. Tripathy, "Simulation of flywheel energy storage system for city buses," Energy Conversion and Management, vol. 33, no. 4, pp. 243 - 250, 1992. [Online]. Available: http://www.sciencedirect.com/science/article/pii/019689049290114C

[141] K. Itani, A. D. Bernardinis, Z. Khatir, and A. Jammal, "Comparative analysis of two hybrid energy storage systems used in a two front wheel driven electric vehicle during extreme start-up and regenerative braking operations," Energy Conversion and Management, vol. 144, pp. 69 - 87, 2017. [Online]. Available: http://www.sciencedirect.com/science/article/pii/S0196890417303461

[142] S. Koohi-Kamali and N. A. Rahim, "Coordinated control of smart microgrid during and after islanding operation to prevent under frequency load shedding using energy storage system," Energy Conversion and Management, vol. 127, 
pp. 623 - 646, 2016. [Online]. Available: http://www.sciencedirect.com/science/ article/pii/S0196890416308512

[143] S. Singh, M. Singh, and S. C. Kaushik, "Feasibility study of an islanded microgrid in rural area consisting of pv, wind, biomass and battery energy storage system," Energy Conversion and Management, vol. 128, pp. 178 - 190, 2016. [Online]. Available: http://www.sciencedirect.com/science/article/pii/S0196890416308366

[144] K. H. Chua, Y. S. Lim, and S. Morris, "Cost-benefit assessment of energy storage for utility and customers: A case study in malaysia," Energy Conversion and Management, vol. 106, pp. 1071 - 1081, 2015. [Online]. Available: http://www.sciencedirect.com/science/article/pii/S0196890415009632

[145] A. Colmenar-Santos, A.-R. Linares-Mena, J. F. Velzquez, and D. Borge-Diez, "Energy-efficient three-phase bidirectional converter for grid-connected storage applications," Energy Conversion and Management, vol. 127, pp. 599 611, 2016. [Online]. Available: http://www.sciencedirect.com/science/article/pii/ S0196890416308378

[146] K. Wu, H. Zhou, S. An, and T. Huang, "Optimal coordinate operation control for windphotovoltaicbattery storage power-generation units," Energy Conversion and Management, vol. 90, pp. 466 - 475, 2015. [Online]. Available: http://www.sciencedirect.com/science/article/pii/S0196890414009959

[147] K. Kusakana, "Feasibility analysis of river off-grid hydrokinetic systems with pumped hydro storage in rural applications," Energy Conversion and Management, vol. 96, pp. 352 - 362, 2015. [Online]. Available: http: //www.sciencedirect.com/science/article/pii/S0196890415002125

[148] J. Devotta and M. Rabbani, "Application of superconducting magnetic energy storage unit in multi-machine power systems," Energy Conversion and Management, vol. 41, no. 5, pp. 493 - 504, 2000. [Online]. Available: http://www.sciencedirect.com/science/article/pii/S0196890499001004

[149] J. Kondoh, I. Ishii, H. Yamaguchi, A. Murata, K. Otani, K. Sakuta, N. Higuchi, S. Sekine, and M. Kamimoto, "Electrical energy storage systems for energy networks," Energy Conversion and Management, vol. 41, no. 17, pp. 1863 1874, 2000. [Online]. Available: http://www.sciencedirect.com/science/article/pii/ S0196890400000285

[150] H. Shayeghi, H. Shayanfar, and A. Jalili, "Load frequency control strategies: A state-of-the-art survey for the researcher," Energy Conversion and Management, 
vol. 50, no. 2, pp. 344 - 353, 2009. [Online]. Available: http://www.sciencedirect. com/science/article/pii/S0196890408003567

[151] S. Pothiya and I. Ngamroo, "Optimal fuzzy logic-based pid controller for loadfrequency control including superconducting magnetic energy storage units," Energy Conversion and Management, vol. 49, no. 10, pp. 2833 2838, 2008. [Online]. Available: http://www.sciencedirect.com/science/article/pii/ S0196890408000952

[152] M. G. Molina, P. E. Mercado, and E. H. Watanabe, "Static synchronous compensator with superconducting magnetic energy storage for high power utility applications," Energy Conversion and Management, vol. 48, no. 8, pp. 2316 2331, 2007. [Online]. Available: http://www.sciencedirect.com/science/article/pii/ S0196890407000714

[153] M. T. Ansari and S. Velusami, "Dual mode linguistic hedge fuzzy logic controller for an isolated winddiesel hybrid power system with superconducting magnetic energy storage unit," Energy Conversion and Management, vol. 51, no. 1, pp. 169 - 181, 2010. [Online]. Available: http://www.sciencedirect.com/science/article/ pii/S0196890409003501

[154] I. Ngamroo, "An optimization technique of robust load frequency stabilizer for superconducting magnetic energy storage," Energy Conversion and Management, vol. 46, no. 18, pp. 3060 - 3090, 2005. [Online]. Available: http: //www.sciencedirect.com/science/article/pii/S0196890405000531

[155] S. Tripathy, "Dynamic simulation of hybrid wind-diesel power generation system with superconducting magnetic energy storage," Energy Conversion and Management, vol. 38, no. 9, pp. 919 - 930, 1997. [Online]. Available: http://www.sciencedirect.com/science/article/pii/S0196890496000933

[156] H. Wen and B. Su, "Operating modes and practical power flow analysis of bidirectional isolated power interface for distributed power systems," Energy Conversion and Management, vol. 111, pp. 229 - 238, 2016. [Online]. Available: http://www.sciencedirect.com/science/article/pii/S0196890415011802

[157] C. Yin, H. Wu, F. Locment, and M. Sechilariu, "Energy management of \{DC $\}$ microgrid based on photovoltaic combined with diesel generator and supercapacitor," Energy Conversion and Management, vol. 132, pp. 14 27, 2017. [Online]. Available: http://www.sciencedirect.com/science/article/pii/ S0196890416310135 
[158] J. P. Torreglosa, P. Garca-Trivio, L. M. Fernndez-Ramirez, and F. Jurado, "Decentralized energy management strategy based on predictive controllers for a medium voltage direct current photovoltaic electric vehicle charging station," Energy Conversion and Management, vol. 108, pp. 1 - 13, 2016. [Online]. Available: http://www.sciencedirect.com/science/article/pii/S0196890415009966

[159] D. Pavkovi, M. Lobrovi, M. Hrgeti, and A. Komljenovi, "A design of cascade control system and adaptive load compensator for battery/ultracapacitor hybrid energy storage-based direct current microgrid," Energy Conversion and Management, vol. 114, pp. 154 - 167, 2016. [Online]. Available: http://www.sciencedirect.com/science/article/pii/S0196890416300280

[160] P. K. Ray, S. R. Mohanty, and N. Kishor, "Proportionalintegral controller based small-signal analysis of hybrid distributed generation systems," Energy Conversion and Management, vol. 52, no. 4, pp. 1943 - 1954, 2011. [Online]. Available: http://www.sciencedirect.com/science/article/pii/S019689041000511X

[161] A. Choudar, D. Boukhetala, S. Barkat, and J.-M. Brucker, "A local energy management of a hybrid pv-storage based distributed generation for microgrids," Energy Conversion and Management, vol. 90, pp. 21 - 33, 2015. [Online]. Available: http://www.sciencedirect.com/science/article/pii/S0196890414009625

[162] J. Jurasz, "Modeling and forecasting energy flow between national power grid and a solarwindpumped-hydroelectricity (pvwtpsh) energy source," Energy Conversion and Management, vol. 136, pp. 382 - 394, 2017. [Online]. Available: http://www.sciencedirect.com/science/article/pii/S0196890417300407

[163] B. K. Paul Denholm, Erik Ela and M. Milligan, "The role of energy storage with renewable electricity generation," Technical Report NREL, vol. PP, no. TP-6A247187, January 2010.

[164] M. Motalleb, M. Thornton, E. Reihani, and R. Ghorbani, "Providing frequency regulation reserve services using demand response scheduling," Energy Conversion and Management, vol. 124, pp. 439 - 452, 2016. [Online]. Available: http://www.sciencedirect.com/science/article/pii/S0196890416306276

[165] "Application of battery-based storage systems in household-demand smoothening in electricity-distribution grids," Energy Conversion and Management, vol. 65, pp. 272 - 284, 2013, global Conference on Renewable energy and Energy Efficiency for Desert Regions 2011. 
[166] T. Ma, C. R. Lashway, Y. Song, and O. Mohammed, "Optimal renewable energy farm and energy storage sizing method for future hybrid power system," in 2014 17th International Conference on Electrical Machines and Systems (ICEMS), Oct 2014, pp. 2827-2832.

[167] N.-K. C. Nair and N. Garimella, "Battery energy storage systems: Assessment for small-scale renewable energy integration," Energy and Buildings, vol. 42, no. 11, pp. 2124 - 2130, 2010. [Online]. Available: http://www.sciencedirect.com/ science/article/pii/S0378778810002185

[168] E. L. Cascio, D. Borelli, F. Devia, and C. Schenone, "Future distributed generation: An operational multi-objective optimization model for integrated small scale urban electrical, thermal and gas grids," Energy Conversion and Management, vol. 143, pp. 348 - 359, 2017. [Online]. Available: http://www.sciencedirect.com/science/article/pii/S0196890417303114

[169] Z. Huang, X. Li, M. Mahboub, K. M. Hanson, V. M. Nichols, H. Le, M. L. Tang, and C. J. Bardeen, "Hybrid moleculenanocrystal photon upconversion across the visible and near-infrared," Nano Letters, vol. 15, no. 8, pp. 5552-5557, 2015, pMID: 26161875. [Online]. Available: http: //dx.doi.org/10.1021/acs.nanolett.5b02130

[170] E. Ackerman, Japan Demoes Wireless Power Transmission for Space-Based Solar Farms, 2015 (accessed August 21, 2017). [Online]. Available: http://spectrum.ieee.org/energywise/green-tech/solar/ japan-demoes-wireless-power-transmission-for-spacebased-solar-farms

[171] L. Wang, T. H. Yeh, W. J. Lee, and Z. Chen, "Benefit evaluation of wind turbine generators in wind farms using capacity-factor analysis and economic-cost methods," IEEE Transactions on Power Systems, vol. 24, no. 2, pp. 692-704, May 2009.

[172] A. Demiroren and U. Yilmaz, "Analysis of change in electric energy cost with using renewable energy sources in gkceada, turkey: An island example," Renewable and Sustainable Energy Reviews, vol. 14, no. 1, pp. 323 - 333, 2010. [Online]. Available: http://www.sciencedirect.com/science/article/pii/S1364032109001348

[173] P. Bajpai, N. P. Prakshan, and N. K. Kishore, "Renewable hybrid stand-alone telecom power system modeling and analysis," in TENCON 2009 - 2009 IEEE Region 10 Conference, Jan 2009, pp. 1-6.

[174] G. O. Cimuca, C. Saudemont, B. Robyns, and M. M. Radulescu, "Control and performance evaluation of a flywheel energy-storage system associated to a variable- 
speed wind generator," IEEE Transactions on Industrial Electronics, vol. 53, no. 4, pp. 1074-1085, June 2006.

[175] J. Kaldellis, P. Koronakis, and K. Kavadias, "Energy balance analysis of a stand-alone photovoltaic system, including variable system reliability impact," Renewable Energy, vol. 29, no. 7, pp. 1161 - 1180, 2004. [Online]. Available: http://www.sciencedirect.com/science/article/pii/S0960148103003884

[176] J. L. Bernal-Agustn, R. Dufo-Lpez, and D. M. Rivas-Ascaso, "Design of isolated hybrid systems minimizing costs and pollutant emissions," Renewable Energy, vol. 31, no. 14, pp. 2227 - 2244, 2006. [Online]. Available: http://www.sciencedirect.com/science/article/pii/S0960148105003356

[177] S. Shaahid and M. Elhadidy, "Economic analysis of hybrid photovoltaicdieselbattery power systems for residential loads in hot regionsa step to clean future," Renewable and Sustainable Energy Reviews, vol. 12, no. 2, pp. 488 - 503, 2008. [Online]. Available: http://www.sciencedirect.com/science/article/pii/S136403210600116X

[178] J. Cotrell, W. Pratt, J. Cotrell, and W. Pratt, "Modelling the feasibility of using fuel cells and hydrogen internal combustion engines in remote renewable energy system,” National Renewable Energy Laboratory (NREL), Tech. Rep., 2003.

[179] A. Gupta, R. P. Saini, and M. P. Sharma, "Design of an optimal hybrid energy system model for remote rural area power generation," in 2007 International Conference on Electrical Engineering, April 2007, pp. 1-6.

[180] G. M. Masters, Renewable and Efficient Electric Power Systems. Hoboken, NJ: John Wiley \& Sons, 2013.

[181] C. S. Kevin I.C. Donaldson, "Renewables portfolio standard 2008-2010 procurement verification," California Energy Commission, Tech. Rep., 2013.

[182] A. Anzalchi and B. Mozafari, "Wind-pv-grid connected hybrid renewable system in kish island," International Review on Modelling and Simulations (I. RE. MO. S.), vol. 4, no. 6, 2011.

[183] S. Karki, M. D. Mann, and H. Salehfar, "Substitution and price effects of carbon tax on co2 emissions reduction from distributed energy sources," in 2006 Power Systems Advanced Metering, Protection, Control, Communication, and Distributed Resources, March 2006, pp. 236-243. 
[184] M. A. Salmani, A. Anzalchi, and S. Salmani, "Virtual power plant: New solution for managing distributed generations in decentralized power systems," in $2010 \mathrm{In}$ ternational Conference on Management and Service Science, Aug 2010, pp. 1-6.

[185] A. K. Srivastava, A. A. Kumar, and N. N. Schulz, "Impact of distributed generations with energy storage devices on the electric grid," IEEE Systems Journal, vol. 6, no. 1, pp. 110-117, March 2012.

[186] S. Moury, M. N. Khandoker, and S. M. Haider, "Feasibility study of solar pv arrays in grid connected cellular bts sites," in 2012 International Conference on Advances in Power Conversion and Energy Technologies (APCET), Aug 2012, pp. 1-5.

[187] "Detailed model of a 100-kw grid-connected pv array and wind farm (dfig phasor model)," MathWorks ${ }^{\circledR}$ SimPower Systems, Jan 2014. [Online]. Available: http://www.mathworks.com/

[188] F. Liu, S. Duan, F. Liu, B. Liu, and Y. Kang, "A variable step size inc mppt method for pv systems," IEEE Transactions on Industrial Electronics, vol. 55, no. 7, pp. 2622-2628, July 2008.

[189] A. Anzalchi and B. Mozafari, "Wind-pv-grid connected hybrid renewable system in kish island," vol. 4, 122011.

[190] M. Berrera, A. Dolara, R. Faranda, and S. Leva, "Experimental test of seven widely-adopted mppt algorithms," in 2009 IEEE Bucharest PowerTech, June 2009, pp. 1-8.

[191] S. Sreekanth and I. J. Raglend, "A comparitive and analytical study of various incremental algorithms applied in solar cell," in 2012 International Conference on Computing, Electronics and Electrical Technologies (ICCEET), March 2012, pp. 452-456.

[192] E. M. Ahmed and M. Shoyama, "Modified adaptive variable step-size mppt basedon single current sensor," in TENCON 2010 - 2010 IEEE Region 10 Conference, Nov 2010, pp. 1235-1240.

[193] W. Xiao and W. G. Dunford, "A modified adaptive hill climbing mppt method for photovoltaic power systems," in 2004 IEEE 35th Annual Power Electronics Specialists Conference (IEEE Cat. No.04CH37551), vol. 3, June 2004, pp. 19571963 Vol.3. 
[194] C. S. Chin, Y. K. Chin, B. L. Chua, A. Kiring, and K. T. K. Teo, "Fuzzy logic based mppt for pv array under partially shaded conditions," in 2012 International Conference on Advanced Computer Science Applications and Technologies (ACSAT), Nov 2012, pp. 133-138.

[195] M. Boztepe, F. Guinjoan, G. Velasco-Quesada, S. Silvestre, A. Chouder, and E. Karatepe, "Global mppt scheme for photovoltaic string inverters based on restricted voltage window search algorithm," IEEE Transactions on Industrial Electronics, vol. 61, no. 7, pp. 3302-3312, July 2014.

[196] T. A. Ocran, J. Cao, B. Cao, and X. Sun, "Artificial neural network maximum power point tracker for solar electric vehicle," Tsinghua Science and Technology, vol. 10, no. 2, pp. 204 - 208, 2005. [Online]. Available: http://www.sciencedirect.com/science/article/pii/S1007021405700559

[197] B. Bendib, F. Krim, H. Belmili, M. F. Almi, and S. Bolouma, "An intelligent mppt approach based on neural-network voltage estimator and fuzzy controller, applied to a stand-alone pv system," in 2014 IEEE 23rd International Symposium on Industrial Electronics (ISIE), June 2014, pp. 404-409.

[198] Syafaruddin, E. Karatepe, and T. Hiyama, "Artificial neural network-polar coordinated fuzzy controller based maximum power point tracking control under partially shaded conditions," IET Renewable Power Generation, vol. 3, no. 2, pp. 239-253, June 2009.

[199] F. Giraud and Z. M. Salameh, "Analysis of the effects of a passing cloud on a gridinteractive photovoltaic system with battery storage using neural networks," IEEE Transactions on Energy Conversion, vol. 14, no. 4, pp. 1572-1577, Dec 1999.

[200] J. Liang, S. K. K. Ng, G. Kendall, and J. W. M. Cheng, "Load signature study part i: Basic concept, structure, and methodology," IEEE Transactions on Power Delivery, vol. 25, no. 2, pp. 551-560, April 2010.

[201] N. Golovanov, G. C. Lazaroiu, M. Roscia, and D. Zaninelli, "Power quality assessment in small scale renewable energy sources supplying distribution systems," Energies, vol. 6, no. 2, pp. 634-645, 2013. [Online]. Available: http://www.mdpi.com/1996-1073/6/2/634

[202] A. Honrubia-Escribano, T. Garcia-Sanchez, E. Gomez-Lazaro, E. Muljadi, and A. Molina-Garcia, "Power quality surveys of photovoltaic power plants: characterisation and analysis of grid-code requirements," IET Renewable Power Generation, vol. 9, no. 5, pp. 466-473, 2015. 
[203] A. R. Oliva, J. C. Balda, D. W. McNabb, and R. D. Richardson, "Power-quality monitoring of a pv generator," IEEE Transactions on Energy Conversion, vol. 13, no. 2, pp. 188-193, Jun 1998.

[204] S. Patra, N. Kishor, S. R. Mohanty, and P. K. Ray, "Power quality assessment in 3-phase grid connected pv system with single and dual stage circuits," International Journal of Electrical Power \& Energy Systems, vol. 75, pp. $275-$ 288, 2016. [Online]. Available: http://www.sciencedirect.com/science/article/pii/ S0142061515003968

[205] A. Khalilnejad, A. Abbaspour, and A. Sarwat, "Multi-level optimization approach for directly coupled photovoltaic-electrolyser system," International Journal of Hydrogen Energy, In Press, pp. -, May 2016. [Online]. Available: http://www.sciencedirect.com/science/article/pii/S0360319915313938

[206] X. Fu, S. Li, and I. Jaithwa, "Implement optimal vector control for lcl-filter-based grid-connected converters by using recurrent neural networks," IEEE Trans. Ind. Electron., vol. 62, no. 7, pp. 4443-4454, July 2015.

[207] D. Yang, X. Ruan, and H. Wu, "A real-time computation method with dual sampling mode to improve the current control performance of the lcl -type gridconnected inverter," IEEE Trans. Ind. Electron., vol. 62, no. 7, pp. 4563-4572, July 2015.

[208] X. Zhang, Y. Wang, C. Yu, L. Guo, and R. Cao, "Hysteresis model predictive control for high-power grid-connected inverters with output lcl filter," IEEE Trans. Ind. Electron., vol. 63, no. 1, pp. 246-256, Jan 2016.

[209] Y. Jiao, S. Lu, and F. Lee, "Switching performance optimization of a high power high frequency three-level active neutral point clamped phase leg," Power Electronics, IEEE Transactions on, vol. 29, no. 7, pp. 3255-3266, July 2014.

[210] W. Wu, P. Geng, X. Wang, Y. Ye, and T. Tang, "A novel control method for dual mode time-sharing cascaded sinusoidal inverter," in Industrial Electronics, 2008. IECON 2008. 34th Annual Conference of IEEE, Nov 2008, pp. 479-483.

[211] N. Ahmed, H. W. Lee, and M. Nakaoka, "Dual-mode time-sharing sinewavemodulation soft switching boost full-bridge one-stage power conditioner without electrolytic capacitor dc link," Industry Applications, IEEE Transactions on, vol. 43, no. 3, pp. 805-813, May 2007. 
[212] S. Saridakis, E. Koutroulis, and F. Blaabjerg, "Optimization of sic-based h5 and conergy-npc transformerless pv inverters," in Energy Conversion Congress and Exposition (ECCE), 2013 IEEE, Sept 2013, pp. 4732-4739.

[213] P. Channegowda and V. John, "Filter optimization for grid interactive voltage source inverters," Industrial Electronics, IEEE Transactions on, vol. 57, no. 12, pp. 4106-4114, Dec 2010.

[214] W. Wu, Y. He, and F. Blaabjerg, "An llcl power filter for single-phase grid-tied inverter," Power Electronics, IEEE Transactions on, vol. 27, no. 2, pp. 782-789, Feb 2012.

[215] J. Bloemink and T. Green, "Reducing passive filter sizes with tuned traps for distribution level power electronics," in Power Electronics and Applications (EPE 2011), Proceedings of the 2011-14th European Conference on, Aug 2011, pp. 1-9.

[216] J. Huang and H. Shi, "A hybrid filter for the suppression of common-mode voltage and differential-mode harmonics in three-phase inverters with cppm," IEEE Transactions on Industrial Electronics, vol. 62, no. 7, pp. 3991-4000, July 2015.

[217] Y. Liu, W. Wu, Y. He, Z. Lin, F. Blaabjerg, and H. S. H. Chung, "An efficient and robust hybrid damper for lcl - or llcl -based grid-tied inverter with strong grid-side harmonic voltage effect rejection," IEEE Trans. Ind. Electron., vol. 63, no. 2, pp. 926-936, Feb 2016.

[218] J. Ji, W. Wu, Y. He, Z. Lin, F. Blaabjerg, and H.-H. Chung, "A simple differential mode emi suppressor for the llcl -filter-based single-phase grid-tied transformerless inverter," Industrial Electronics, IEEE Transactions on, vol. 62, no. 7, pp. 41414147, July 2015.

[219] D. Pan, X. Ruan, C. Bao, W. Li, and X. Wang, "Optimized controller design for lcl -type grid-connected inverter to achieve high robustness against grid-impedance variation," IEEE Trans. Ind. Electron., vol. 62, no. 3, pp. 1537-1547, March 2015.

[220] "Ieee recommended practice and requirements for harmonic control in electric power systems," IEEE Std 519-2014 (Revision of IEEE Std 519-1992), pp. 1-29, June 2014.

[221] "Ieee application guide for ieee std $1547(\mathrm{tm})$, ieee standard for interconnecting distributed resources with electric power systems," IEEE Std 1547.2-2008, pp. 1217, April 2009. 
[222] B. Bolsens, K. De Brabandere, J. Van den Keybus, J. Driesen, and R. Belmans, "Model-based generation of low distortion currents in grid-coupled pwm-inverters using an lcl output filter," Power Electronics, IEEE Transactions on, vol. 21, no. 4, pp. 1032-1040, July 2006.

[223] G. r. Zhu, H. Wang, B. Liang, S. C. Tan, and J. Jiang, "Enhanced single-phase full-bridge inverter with minimal low-frequency current ripple," IEEE Trans. Ind. Electron., vol. 63, no. 2, pp. 937-943, Feb 2016.

[224] G. Grandi, J. Loncarski, and O. Dordevic, "Analysis and comparison of peak-topeak current ripple in two-level and multilevel pwm inverters," IEEE Trans. Ind. Electron., vol. 62, no. 5, pp. 2721-2730, May 2015.

[225] S. Sridharan and P. T. Krein, in 2015 IEEE International Electric Machines Drives Conference (IEMDC).

[226] K. Hatua, A. K. Jain, D. Banerjee, and V. T. Ranganathan, "Active damping of output filter resonance for vector-controlled vsi-fed ac motor drives," IEEE Transactions on Industrial Electronics, vol. 59, no. 1, pp. 334-342, Jan, 2012.

[227] K. Jalili and S. Bernet, "Design of lcl filters of active-front-end two-level voltagesource converters," IEEE Trans. Ind. Electron., vol. 56, no. 5, pp. 1674-1689, May 2009.

[228] X. Zheng, L. Xiao, Y. Lei, and Z. Wang, "Optimisation of lcl filter based on closedloop total harmonic distortion calculation model of the grid-connected inverter," IET Power Electronics, vol. 8, no. 6, pp. 860-868, 2015.

[229] A. Singh, A. K. Kaviani, and B. Mirafzal, "On dynamic models and stability analysis of three-phase phasor pwm-based csi for stand-alone applications," IEEE Trans. Ind. Electron., vol. 62, no. 5, pp. 2698-2707, May 2015.

[230] C. A. Busada, S. G. Jorge, and J. A. Solsona, "Full-state feedback equivalent controller for active damping in lcl -filtered grid-connected inverters using a reduced number of sensors," IEEE Trans. Ind. Electron., vol. 62, no. 10, pp. 5993-6002, Oct 2015.

[231] M. Moghaddami, A. Anzalchi, and A. Sarwat, "Single-stage three-phase ac-ac matrix converter for inductive power transfer systems," IEEE Trans. Ind. Electron. , Early Access Article, vol. PP, no. 99, pp. 1-1, May 2016. 
[232] A. A. Rockhill, M. Liserre, R. Teodorescu, and P. Rodriguez, "Grid-filter design for a multimegawatt medium-voltage voltage-source inverter," IEEE Transactions on Industrial Electronics, vol. 58, no. 4, pp. 1205-1217, April 2011.

[233] M. Davari and Y. A. R. I. Mohamed, "Dynamics and robust control of a gridconnected vsc in multiterminal de grids considering the instantaneous power of dc- and ac-side filters and dc grid uncertainty," IEEE Transactions on Power Electronics, vol. 31, no. 3, pp. 1942-1958, March 2016.

[234] Masoud Davari, "Dynamics, Robust Control, and Power Management of Voltage-Source Converters in Hybrid Multiterminal AC/DC Grids," Ph.D. dissertation, University of Alberta, Edmonton, Jan. 2016. [Online]. Available: https://era.library.ualberta.ca

[235] M. Davari and Y. A. R. I. Mohamed, "Variable-structure-based nonlinear control for the master vsc in dc-energy-pool multiterminal grids," IEEE Transactions on Power Electronics, vol. 29, no. 11, pp. 6196-6213, Nov 2014.

[236] M. Davarii and Y. A. R. I. Mohamed, "Robust multi-objective control of vsc-based dc-voltage power port in hybrid ac-dc multi-terminal micro-grids," IEEE Transactions on Smart Grid, vol. 4, no. 3, pp. 1597-1612, Sept 2013.

[237] Q.-C. Zhong and G. Weiss, "Synchronverters: Inverters that mimic synchronous generators," Industrial Electronics, IEEE Transactions on, vol. 58, no. 4, pp. 12591267, April 2011.

[238] H. P. Beck and R. Hesse, "Virtual synchronous machine," in 2007 9th International Conference on Electrical Power Quality and Utilisation, Oct 2007, pp. 1-6.

[239] M. Farhadi and O. Mohammed, "Adaptive energy management in redundant hybrid dc microgrid for pulse load mitigation," IEEE Transactions on Smart Grid, vol. 6, no. 1, pp. 54-62, Jan 2015.

[240] A. Bazaei, S. Moheimani, and Y. Yong, "Improvement of transient response in signal transformation approach by proper compensator initialization," Control Systems Technology, IEEE Transactions on, vol. 22, no. 2, pp. 729-736, March 2014.

[241] S. D'Arco and J. Suul, "Equivalence of virtual synchronous machines and frequency-droops for converter-based microgrids," Smart Grid, IEEE Transactions on, vol. 5, no. 1, pp. 394-395, Jan 2014. 
[242] Y. Hirase, K. Abe, K. Sugimoto, and Y. Shindo, "A grid-connected inverter with virtual synchronous generator model of algebraic type," Electrical Engineering in Japan, vol. 184, no. 4, pp. 10-21, 2013. [Online]. Available: http://dx.doi.org/10.1002/eej.22428

[243] R. Hesse, D. Turschner, and H.-P. Beck, "Micro grid stabilization using the virtual synchronous machine (visma)," vol. 1, pp. 676-681, 042009.

[244] Y. Chen, R. Hesse, D. Turschner, and H.-P. Beck, "Dynamic properties of the virtual synchronous machine (visma)," 052011.

[245] X. Meng, Z. Liu, J. Liu, T. Wu, S. Wang, and B. Liu, "Comparison between virtual synchronous generator and droop controlled inverter," in 2016 IEEE 2nd Annual Southern Power Electronics Conference (SPEC), Dec 2016, pp. 1-6.

[246] J. Driesen and K. Visscher, "Virtual synchronous generators," in 2008 IEEE Power and Energy Society General Meeting - Conversion and Delivery of Electrical Energy in the 21st Century, July 2008, pp. 1-3.

[247] V. Karapanos, S. de Haan, and K. Zwetsloot, "Real time simulation of a power system with vsg hardware in the loop," in IECON 2011 - 37th Annual Conference of the IEEE Industrial Electronics Society, Nov 2011, pp. 3748-3754.

[248] M. Albu, J. Diaz, V. Thong, R. Neurohr, D. Federenciuc, M. Popa, and M. Calin, "Measurement and remote monitoring for virtual synchronous generator design," in 2010 IEEE International Workshop on Applied Measurements for Power Systems, Sept 2010, pp. 7-11.

[249] K. Visscher and S. W. H. D. Haan, "Virtual synchronous machines (vsg s) for frequency stabilisation in future grids with a significant share of decentralized generation," in CIRED Seminar 2008: SmartGrids for Distribution, June 2008, pp. $1-4$.

[250] T. Shintai, Y. Miura, and T. Ise, "Reactive power control for load sharing with virtual synchronous generator control," in Proceedings of The 7th International Power Electronics and Motion Control Conference, vol. 2, June 2012, pp. 846853.

[251] K. Sakimoto, Y. Miura, and T. Ise, "Stabilization of a power system including inverter-type distributed generators by a virtual synchronous generator," vol. 132, pp. 341-349, 012012. 
[252] — - "Stabilization of a power system with a distributed generator by a virtual synchronous generator function," in 8th International Conference on Power Electronics - ECCE Asia, May 2011, pp. 1498-1505.

[253] M. P. N. van Wesenbeeck, S. W. H. de Haan, P. Varela, and K. Visscher, "Grid tied converter with virtual kinetic storage," in 2009 IEEE Bucharest PowerTech, June 2009, pp. 1-7.

[254] M. Albu, M. Calin, D. Federenciuc, and J. Diaz, "The measurement layer of the virtual synchronous generator operation in the field test," in 2011 IEEE International Workshop on Applied Measurements for Power Systems (AMPS), Sept 2011, pp. 85-89.

[255] X. Wang, M. Yue, and E. Muljadi, "Pv generation enhancement with a virtual inertia emulator to provide inertial response to the grid," in Energy Conversion Congress and Exposition (ECCE), 2014 IEEE, Sept 2014, pp. 17-23.

[256] S. K. Kollimalla, M. K. Mishra, and N. L. Narasamma, "Design and analysis of novel control strategy for battery and supercapacitor storage system," IEEE Transactions on Sustainable Energy, vol. 5, no. 4, pp. 1137-1144, Oct 2014.

[257] H. Saadat, Power system analysis. New York, NY: McGraw-Hill, 1999.

[258] M. Eremia and M. Shahidehpour, Power System Modeling and Control. WileyIEEE Press, 2013, pp. 7-8. [Online]. Available: http://ieeexplore.ieee.org/xpl/ articleDetails.jsp?arnumber $=6480479$

[259] A. Ellis, D. Schoenwald, J. Hawkins, S. Willard, and B. Arellano, "Pv output smoothing with energy storage," in 2012 38th IEEE Photovoltaic Specialists Conference, June 2012, pp. $001523-001528$.

[260] NRECAs Renewable and Distributed Generation work group , "Energy storage use case: Pv smoothing," 2016.

[261] R. Sathishkumar, S. Kollimalla, and M. Mishra, "Dynamic energy management of micro grids using battery super capacitor combined storage," in India Conference (INDICON), 2012 Annual IEEE, Dec 2012, pp. 1078-1083.

[262] M. Farhadi and O. Mohammed, "Real-time operation and harmonic analysis of isolated and non-isolated hybrid dc microgrid," Industry Applications, IEEE Transactions on, vol. 50, no. 4, pp. 2900-2909, July 2014. 
[263] M. Farhadi, "Hybrid energy storage implementation in dc and ac power system for efficiency, power quality and reliability improvements," Ph.D. dissertation, Florida International University, 2016.

[264] V. Musolino, L. Piegari, and E. Tironi, "New full-frequency-range supercapacitor model with easy identification procedure," IEEE Transactions on Industrial Electronics, vol. 60, no. 1, pp. 112-120, Jan 2013.

[265] R. Dougal, L. Gao, and S. Liu, "Ultracapacitor model with automatic order selection and capacity scaling for dynamic system simulation," Journal of Power Sources, vol. 126, no. 1, pp. 250 - 257, 2004. [Online]. Available: http://www.sciencedirect.com/science/article/pii/S0378775303009169

[266] M. Uzunoglu, O. Onar, and M. Alam, "Modeling, control and simulation of a pv/fc/uc based hybrid power generation system for stand-alone applications," Renewable Energy, vol. 34, no. 3, pp. 509 - 520, 2009. [Online]. Available: http://www.sciencedirect.com/science/article/pii/S0960148108002498

[267] Z. M. Salameh, M. A. Casacca, and W. A. Lynch, "A mathematical model for leadacid batteries," IEEE Transactions on Energy Conversion, vol. 7, no. 1, pp. 93-98, Mar 1992.

[268] C. M. Shepherd, "Design of primary and secondary cells - part 2. an equation describing battery discharg," Journal of Electrochemical Society, vol. 112, no. 1, pp. 657-664, July 1965.

[269] N. Mendis, K. M. Muttaqi, and S. Perera, "Active power management of a super capacitor-battery hybrid energy storage system for standalone operation of dfig based wind turbines," in 2012 IEEE Industry Applications Society Annual Meeting, Oct 2012, pp. 1-8.

[270] S. Kolluri and N. L. Narasamma, "Analysis, modeling, design and implementation of average current mode control for interleaved boost converter," in 2013 IEEE 10th International Conference on Power Electronics and Drive Systems (PEDS), April 2013, pp. 280-285.

[271] Y. Jin, J. Xu, G. Zhou, and C. Mi, "Small-signal modeling and analysis of improved digital peak current control of boost converter," in 2009 IEEE 6th International Power Electronics and Motion Control Conference, May 2009, pp. 326-330.

[272] MATLAB 2017b Documentation; [user's Guide]. MathWorks, 2017. [Online]. Available: https://www.mathworks.com/help/simulink/slref/ratelimiter.html 
VITA

\section{ARASH ANZALCHI}

September 07, 1982

$1996-2000$

2000-2007

2007-2011

2014-2018

2017
Born, Tehran, Iran

National Diploma, Mathematics and Physics Alborz High School

Tehran, Iran

B.S., Electrical Engineering

K.N. Toosi University of Technology

Tehran, Iran

M.S., Electrical Engineering

Science and Research Branch, Islamic Azad University

Tehran, Iran

Doctoral Candidate, Electrical Engineering Florida International University

Miami, Florida

Dissertation Year Fellowship

Florida International University

\section{PUBLICATIONS AND PRESENTATIONS}

[1] Arash Anzalchi, Arif Sarwat, "Overview of technical specifications for gridconnected photovoltaic systems", in Energy Conversion and Management, Volume 152, 15 November 2017, Pages 312-327, ISSN 0196-8904. (impact factor: 5.589)

[2] A. Anzalchi, M. Moghaddami, A. Moghadasi, M. M. Pour and A. I. Sarwat, "Design and Analysis of a Higher Order Power Filter for Grid-Connected Renewable Energy Systems," in IEEE Transactions on Industry Applications, vol. 53, no. 5, pp. 4149-4161, Sept.-Oct. 2017. doi: 10.1109/TIA.2017.2712783. (impact factor: 2.937)

[3] A. Anzalchi, M. Moghaddami, A. Moghaddasi, A. I. Sarwat and A. K. Rathore, ”A New Topology of Higher Order Power Filter for Single-Phase Grid-Tied Voltage-Source Inverters," in IEEE Transactions on Industrial Electronics, vol. 63, no. 12, pp. 7511 7522, Dec. 2016. doi: 10.1109/TIE.2016.2594222. (impact factor: 7.168) 234

[4] M. Moghaddami, A. Anzalchi and A. I. Sarwat, "Single-Stage Three-Phase ACAC Matrix Converter for Inductive Power Transfer Systems," in IEEE Transactions on Industrial Electronics, vol. 63, no. 10, pp. 6613-6622, Oct. 2016. doi:

10.1109/TIE.2016.2563408. (impact factor: 7.168) 
[5] Arash Anzalchi, Aditya Sundararajan, Longfei Wei, Amir Moghadasi, Arif Sarwat, "Future Directions to the Application of Distributed Fog Computing in Smart Grid Systems", in Smart Grid Analytics for Sustainability and Urbanization Accepted by IGI global Publications, October 2017.

[6] A. Anzalchi, A. Sundararajan, A. Moghadasi, A. Sarwat, "Power Quality and Voltage Profile Analyses of High Penetration Grid-tied Photovoltaics: A Case Study", Accepted in IEEE Industrial Application Annual Meeting, October 2017.

[7] M. M. Pour, A. Anzalchi and A. Sarwat, "A review on cyber security issues and mitigation methods in smart grid systems," SoutheastCon 2017, Charlotte, NC, 2017, pp. 1-4. doi: 10.1109/SECON.2017.7925278

[8] A. Anzalchi, M. M. Pour and A. Sarwat, "A combinatorial approach for addressing intermittency and providing inertial response in a grid-connected photovoltaic system," 2016 IEEE Power and Energy Society General Meeting (PESGM), Boston, MA, 2016, pp. 1-5. doi: 10.1109/PESGM.2016.7742056

[9] A. Moghadasi, M. Moghaddami, A. Anzalchi, A. Sarwat and O. A. Mohammed, "Prioritized coordinated reactive power control of wind turbin involving STATCOM using multi-objective optimization," 2016 IEEE/IAS 52nd Industrial and Commercial Power Systems Technical Conference (I\&CPS), Detroit, MI, 2016, pp. 1-9. doi: 10.1109/ICPS.2016.7490223

[10] M. Moghaddami, A. Anzalchi and A. I. Sarwat, "Finite element based design optimization of magnetic structures for roadway inductive power transfer systems," 2016 IEEE Transportation Electrification Conference and Expo (ITEC), Dearborn, MI, 2016, pp. 1-6. doi: 10.1109/ITEC.2016.7520200

[11] M. Moghaddami, A. Anzalchi, A. Moghadasi and A. Sarwat, "Pareto optimization of circular power pads for contactless electric vehicle battery charger," 2016 IEEE Industry Applications Society Annual Meeting, Portland, OR, 2016, pp. 1-6.

doi: 10.1109/IAS.2016.7731853.235

[12] A. Anzalchi, M. Moghaddami, A. Moghaddasi, M. M. Pour and A. Sarwat, "A modified higher order power filter for grid-connected renewable energy systems," 2016 IEEE/IAS 52nd Industrial and Commercial Power Systems Technical Conference (I\&CPS), Detroit, MI, 2016, pp. 1-9. doi: 10.1109/ICPS.2016.7490217.

[13] A. Anzalchi and A. Sarwat, "Analysis of Carbon Tax as an Incentive Toward Building Sustainable Grid with Renewable Energy Utilization,” 2015 Seventh Annual IEEE Green Technologies Conference, New Orleans, LA, 2015, pp. 103-109. doi: 10.1109/GREENTECH.2015.42. 